\title{
Politische Aktion als Bildungserfahrung. Analyse
}

\subsection{Politisches Bewusstsein - zwei Fallbeispiele}

Für die im Folgenden zusammengefasste exemplarische Darstellung der vielfältigen Vorstellungen und Sinnbildungen der politisch aktiven Schüler*innen werden ihre Deutungsmuster zu den Begriffen Politik und Demokratie skizzenhaft dargestellt. Das Kapitel verfolgt nicht den Anspruch einer vollumfänglichen Analyse der Vorstellungen oder gar einer politikdidaktischen Rekonstruktion, die sich durch die Schritte (1) Erfassung der Vorstellungen, (2) Zielklärung, (3) Fachliche Klärung und (4) Didaktische Strukturierung auszeichnet. ${ }^{1}$ Ziel ist es eher, die Denkmuster exemplarisch zu skizzieren, um einen Eindruck von den vielfältigen Deutungen der Begriffe „Politik“ und „Demokratie“ zu vermitteln. Im Mittelpunkt der vorliegenden Studie und damit auch des Leitfadens für die Interviews standen die (Selbst)Bildungserfahrungen der Schüler*innen in der politischen Aktion. Im Kontext der problemzentrierten Interviews wurde aber auch immer nach den Vorstellungen der Schüler*innen zu den Konzepten „Demokratie“ und „Politik“ gefragt. Dies bildete in der Regel den erzählauffordernden Einstieg in die Interviews. Auch diese Aussagen der Schüler*innen wurden in einem deduktiv-induktiven Verfahren kodiert und nach den Kriterien der qualitativen Inhaltsanalyse ausgewertet. In einem wechselseitigen Abgleich

\footnotetext{
${ }^{1}$ Die in diesem Unterkapitel zusammengefassten Erkenntnisse zu den Vorstellungen, sind hier nur als Einblick in diese Untersuchung aufgenommen. Ausführlich wird die Perspektive der Vorstellungsforschung in Bezug auf reale Partizipationserfahrungen in einem von mir verantworteten Teil des Fachaufsatzes „Politikdidaktische Rekonstruktion und Bürgerbewusstsein. Eine exemplarische Aufarbeitung demokratiebezogener Vorstellungen." (Vajen u. a. 2021) beschrieben. Dort wird auch eine politikdidaktische Rekonstruktion der demokratiebezogenen Vorstellung vorgenommen.
} 
der subjektiven Vorstellungen der interviewten Personen und des fachwissenschaftlichen Diskurses wurde ein übertragbares Kategoriensystem entwickelt. Im Folgenden wird dabei hauptsächlich auf die Demokratie-Trias (Demokratie als Lebens-, Gesellschafts- und Herrschaftsform) von Gerhard Himmelmann (2007) rekurriert. Als Fallbeispiele wurden LEA und JOHANNES ausgewählt. Beide eint, dass sie an ihren jeweiligen Schulen Freiräume für das politische Engagement geschaffen haben und in selbstverwalteten Arbeitsgemeinschaften aktiv sind. Dennoch weisen beide sehr unterschiedliche Vorstellungen von dem Konzept „Demokratie“" auf.

Für LEA bietet die Demokratie für alle Menschen die Möglichkeit, sich an Entscheidungsprozessen zu beteiligen, die sie selbst letztendlich auch betreffen. Diese Art der demokratischen Entscheidung ist dabei nicht allein auf das politische System zu beziehen, sondern findet sich auch in zwischenmenschlichen Aushandlungsprozessen in ihrem eigenen Umfeld wieder, sowohl in ihrem Freundeskreis als auch in ihrer Schule. Sie betont hierbei allerdings, dass diese Vorzüge der Demokratie nicht selbstverständlich seien. Nur wenn sich alle dessen bewusst seien und aktiv mitwirkten an demokratischen Prozessen, könne sie vor Angriffen geschützt werden. Demokratie ist für LEA etwas, das

Wir irgendwie als selbstverständlich ansehen, aber es eigentlich gar nicht selbstverständlich ist, und dass eigentlich man viel mehr sich bewusstmachen sollte, was für ein Glück Demokratie ist, weil jeder selbst mitmachen kann und sich jeder irgendwie engagieren kann und jeder selbst mitmachen kann und das ist ja eigentlich irgendwie etwas Tolles und Schönes. (LEA: 198-202)

Das Konzept der Partizipationsmöglichkeit in Form von Wahlen und Abstimmungen ist dabei zentral für LEA und ihre positive Besetzung des Begriffes der Demokratie. Die Schülerin bezieht sich hier allerdings nicht ausschließlich auf das politische System, sondern es handelt sich in ihrer Vorstellung auch um ein Wertekonstrukt, welches das alltägliche Handeln der Individuen positiv prägen kann. Beispielhaft führt sie hier unter anderem die im Freundeskreis zu treffende Entscheidung an, was man in der Freizeit machen möchte:

Wenn man in der Freizeit mit seinen Freunden ist und überlegt, wollen wir was essen, dann haben alle Hunger und dann steht irgendwie keine Ahnung Pizza und Currywurst zur Auswahl und dann kann man ja auch abstimmen, das ist ja auch Demokratie. (LEA: 179-182)

Diese Vorstellungen sind dabei eng verknüpft mit ihrem Konzept von Politik und Demokratie, die einerseits auf einer systemischen Ebene und andererseits 
im sozialen Nahraum stattfinden. Politik und Demokratie sind für LEA sowohl gekennzeichnet durch einen großen Rahmen des Politischen, also durch das, „was unsere Regierung an Politik durchführt" (LEA: 12), aber zugleich auch von demokratischen Akten, wie der Wahl der Klassensprecher*innen oder die Verhandlungen mit der eigenen Familie über das Taschengeld, die im lebensweltlichen Nahraum anzusiedeln sind. Auch hier zeigt sich wieder eine Differenzierung zwischen einer etwas abstrakten systemischen Ebene und einer lebensweltlichen und alltäglichen Ebene. LEA versteht Demokratie als Teil des lebensweltlichen Alltags, als zivilgesellschaftliche Verpflichtung und als Herrschaftsform (Himmelmann 2013).

In Bezug auf das Demokratische als unhintergehbarer Kern (Haarmann u. a. 2020) bezeichnet LEA Demokratie als Glücksfall und Errungenschaft, die es gegen Angriffe zu verteidigen gilt. Sie betont hierbei vor allem den Wert der Beteiligungsrechte aller Menschen. Sie formuliert damit ein Grundvertrauen in die Demokratie, welches durch eine Auseinandersetzung mit den verfassungsrechtlich verankerten Teilhaberechten der Bürger*innen gefestigt werden kann. LEA sieht die Demokratie vor allem durch Angriffe jener gefährdet, die die Pfeiler der Demokratie, wie beispielsweise die Wahrung von Grund- und Menschenrechten, infrage stellen. Unberücksichtigt bleibt bei ihren Vorstellungen die Tatsache, dass auch innerhalb des bestehenden politischen Systems ungleiche Macht- und Herrschaftsverhältnisse manifestiert werden und auch ohne offene Angriffe auf die Prinzipien der freiheitlichen Demokratie die damit verbundenen Werte gefährdet sein können. Das von ihr stark betonte partizipatorische Element spielt auch eine wichtige Rolle bei ihren Vorstellungen hinsichtlich der Gefahren für die Demokratie. Sie sieht vor allem die Gefahr in einer zu geringen Beteiligung der von ihr als gemäßigt angesehenen Mehrheit der Bürger*innen, was eine überproportionale Repräsentation rechtspopulistischer Meinung zur Folge habe. Die Schülerin verweist darauf, dass es die Demokratie als Herrschaftsform ermögliche, dass radikale Positionen einer Minderheit großen politischen und gesellschaftlichen Raum einnehmen könnten, vor allem dann, wenn sich die schweigende Mehrheit aus dem politischen Raum zurückziehe:

Hinterher wählen nur die, die halt wirklich irgendwie diese rechtspopulistische Meinung vertreten, weil gerade die sich unzufrieden fühlen und gerade die irgendwie was machen wollen. Und am Ende wird irgendein Rechtspopulist Präsident und das ist halt eine Gefahr von Demokratie, wenn einfach nicht alle mitmachen. (LEA: 237-241) 
Diese wahrgenommenen Gefahren rekurrieren primär auf die systemische Ebene der Demokratie und verdeutlichen die normative Dimension politischer Vorstellungen. Grundsätzlich wird dieser Aspekt von der Schülerin aber eher als eine aktuelle Entwicklung angesehen, die nicht die grundlegenden positiven Eigenschaften der Demokratie beeinflusst, sondern eher als Notwendigkeit für eine kontinuierliche Beteiligung der Mehrheitsgesellschaft angesehen wird.

Gefahren für die oder vielmehr durch die Demokratie werden auch von JOHANNES beschrieben. Allerdings nehmen diese hier eine deutlich zentralere Rolle ein und sind mit anderen Konzepten verknüpft als bei LEA. Das von ihm zentral mit der Vorstellung von Demokratie verbundene Konzept ist dabei Macht. Der Schüler betont, dass Demokratie die Kumulation von Macht in den Händen weniger ermögliche, wenngleich dieser Prozess dennoch vom Volk legitimiert würde. Dies macht er daran fest, dass sich

ein Individuum über die anderen stellt, egal in welchem System. So führt ein Kanzler zum Beispiel mehr Macht aus als jemand, der sich in keiner Partei engagiert. Das ist vom System so vorgesehen und das ist eben an sich nicht richtig. (JOHANNES: 47-50)

Dies führe dann unweigerlich zur Einschränkung der Freiheit vieler Individuen. Demokratie erwecke den Anschein eines freiheitlichen Systems, verhindere aber die reale Freiheit des Einzelnen. Das grundlegende Problem der ungleichen Verteilung von Macht schreibt JOHANNES nicht nur aber auch der Demokratie zu. In seinen Augen führt das zu folgendem Effekt:

Die, [die] keinerlei Macht ausüben, die werden eben in ihrer persönlichen Freiheit und auch in ihrer persönlichen Entfaltung dadurch unterdrückt, egal wie. So lange immer irgendwer Macht ausübt, wird das immer so sein. (JOHANNES: 52-55)

Das Phänomen ungleich verteilter Macht durchziehe aber nicht nur das politische System Demokratie, sondern präge auch die demokratische Gesellschaft und dabei vor allem das Wirtschaftssystem. In den Vorstellungen des Schülers ist das Wirtschaftssystem unmittelbar mit dem politischen System verknüpft. Die durch das kapitalistische Wirtschaftssystem hervorgebrachten Ungleichheiten würden sich dadurch manifestieren, dass die wirtschaftlichen Freiheiten der individuellen Freiheit übergeordnet würden.

Man braucht Mittel, um die individuelle Freiheit durchzusetzen, eben Zahlungsmittel. Deshalb wird die menschliche Kreativität in diesem wirtschaftlichen Sektor gelenkt und entwickelt sich darauf, wie sie am besten Leute ausbeuten kann und den eigenen Profit am meisten steigern kann. (JOHANNES: 185-188) 
Demokratie ist für den Schüler dadurch gekennzeichnet, dass sie auch im Wirtschaftssystem ungleiche Machtverteilung ermögliche, indem sie wenigen großen Unternehmen eine marktbestimmende Position erlaube. JOHANNES kommt daher zu dem Schluss, dass aufgrund der grundsätzlich ungeeigneten Struktur der Demokratie, ethische Grundbedürfnisse wie der Wunsch nach Freiheit und Gerechtigkeit in der Demokratie nicht erfüllt werden können. Das Konzept der Macht und der ungleichen Verteilung von Macht spielt bei ihm auch bei weiteren Vorstellungen eine zentrale Rolle, beispielsweise beim Machtmonopol des Staates sowie bei gesamtgesellschaftlichen Zukunftsvorstellungen. Die Demokratievorstellung von JOHANNES steht LEAs Demokratiekonzept diametral gegenüber. JOHANNES verbindet Demokratie primär mit der Herrschafts- und Regierungsform. Er zweifelt daran, dass Demokratie gleiche Teilhabe für alle ermöglicht. Vielmehr geht er davon aus, dass die kollektiven Freiheitsrechte der Demokratie zu ungleich verteilten Ressourcen führen und dies unweigerlich mit der Ausübung von Macht einzelner Menschen und zur Unterdrückung vieler führt. Demokratie wird von JOHANNES vor allem als politisches System verstanden, welches die Grundlage für wirtschaftspolitische Entwicklungen legt, die auf Prinzipien von Macht und Ausbeutung beruhen. Die Konzepte des Wirtschaftssystems und der Demokratie als Herrschaftssystem sind hier eng verknüpft.

Für die politische Bildung ist die Grundhaltung gegenüber der Demokratie als Herrschaftsform ein Lernanlass, den es aufzugreifen gilt. Hierbei wäre in einem ersten Schritt empfehlenswert, aufzuzeigen, dass Kritik an bestehenden Verhältnissen erwünscht ist, solange bei den Überlegungen Grund- und Menschenrechte nicht zur Disposition stehen. Anschlussfähig sind die Vorstellungen von JOHANNES beispielsweise an den aktuellen Diskursen aus der Politikwissenschaft um den Begriff der Postdemokratie (Crouch 2015).

JOHANNES verbindet das gesellschaftspolitische Element der Demokratie in erster Linie mit einem ökonomischen Netzwerk, geprägt von wenigen, aber einflussreichen Unternehmen. Es bestätigt seine teils fatalistische Perspektive und wird gefestigt durch die Vorstellung, dass Demokratie wirtschaftliche Freiheiten vor individuelle Freiheiten stellt und damit den Boden für eine ausbeuterische Wirtschaftsordnung bereitet. Die Kumulation von wirtschaftlicher Macht sei auch in einer freien Gesellschaft möglich, solange die Menschen abhängig davon sind, dass ihre Grundbedürfnisse in einem Tauschgeschäft erfüllt werden.

Beide Befragten verbinden mit Demokratie als Herrschaftsform vor allem die Merkmale einer repräsentativen parlamentarischen Demokratie, die Teilnahme an Wahlen und die Möglichkeit, mittelbar oder unmittelbar Einfluss auf Entscheidungsträger*innen zu nehmen. JOHANNES betont dabei, dass in dem mit der repräsentativen Demokratie verbundenen Merkmal der Herrschaft weniger 
Menschen über viele das Grundprinzip ungleich verteilter Macht manifestiert wird.

Dieser kurze Einblick in die Demokratievorstellungen einer Mittelstufenschülerin und eines Oberstufenschülers zeigen die Vielfalt der bestehenden Denkmuster. An dieser Stelle soll mit den Fallbeispielen LEA und JOHANNES nur ein Einblick in die vielfältigen Vorstellungskonzepte der Jugendlichen gegeben werden. Eine weitergehende Analyse böte sich im Zuge einer Sekundäranalyse des Materials an.

Der Schwerpunkt der vorliegenden Arbeit liegt allerdings nicht auf den Demokratievorstellungen, sondern auf den konkreten Bildungserfahrungen der Schüler*innen im Kontext realer politischer Aktionen. Dafür gilt es, auch die Motivation für die politische Teilhabe in unkonventionellen Aktionsformen zu eruieren. Dafür werden im folgenden Kapitel die motivationalen Faktoren herausgearbeitet.

\subsection{Motivationale Faktoren}

Im Folgenden werden die Erfahrungsberichte der interviewten Schüler*innen unter Berücksichtigung der verschiedenen Dimensionen des politischen Lernens sortiert und strukturiert, mit dem Ziel, politische (Selbst)Bildungserfahrungen aus den Interviews zu rekonstruieren. Davor gilt es allerdings, den Blick auf die Motivation für das selbstbestimmte politische Engagement der Jugendlichen zu richten. Dafür werden verschiedene Faktoren wie das politische Interesse, das Bedürfnis aktiv zu werden, Sozialisation, Gruppendynamik und das politische Bewusstsein berücksichtigt.

Als maßgeblich für das politische Engagement der interviewten Jugendlichen erweisen sich Sozialisationsfaktoren und das soziale Umfeld. Nur MICHAEL verweist explizit darauf, dass seine Eltern nicht politisch sind (MICHAEL: 331). Die meisten befragten Jugendlichen betonen allerdings, dass Politik im Elternhaus eine Rolle spielt. Dabei zeigen sich zwei unterschiedliche Effekte. Für BEN ist seine Politisierung auch verbunden mit einer Distanzierung zu den politischen Positionen und Haltungen seiner Eltern:

Aber mit mehr Alter fängt man so an, sich davon zu distanzieren. Ich glaube einfach, dass das auch so ein bisschen jugendlicher Trotz, ist, sich so ein bisschen gegen seine Eltern aufstellen zu wollen. Und damit fängt man auch irgendwie an, sich mit anderen Sachen zu beschäftigen. (BEN: 181-185) 
JOHANNES berichtet gar davon, dass er sich mit seinen Eltern zerstritten hat. Seinen Schilderungen zufolge endete es in einem „Psychokrieg“ (JOHANNES: 235). Dies führt JOHANNES auch auf den Prozess seiner Politisierung zurück. Wenngleich mehrere Jugendliche davon berichten, dass sie politisch andere Positionen vertreten als ihre Eltern, stellt ein derart weitreichender Bruch mit den eigenen Eltern eher eine Ausnahme dar. Insgesamt kann konstatiert werden, dass die eigene Familie und das soziale Umfeld einen prägenden Einfluss auf die eigene Politisierung haben und damit auch auf die Motivation, sich politisch zu engagieren. Die Faktoren, die diesen Effekt begünstigen, sind allerdings sehr unterschiedlich. FRANZISKA betont, dass politische Debatten zum Alltag in ihrer Familie gehörten. Kritik an bestehenden Verhältnissen sei dabei selbstverständlicher Teil der Diskurse. FRANZISKA hat aber nie verstanden, warum aus der Kritik bei ihrer Familie nie die Bereitschaft erwuchs, sich politisch einzubringen (FRANZISKA 493: „Aber niemand kriegt seinen Arsch vom Sofa entfernt irgendwie.").

Ganz anders schildert HELGE den Einfluss seiner Familie auf das eigene politische Engagement. „Ich glaube, es kommt teilweise vom Elternhaus. “ (HELGE: 200) Seine Eltern waren schon immer politisch aktiv und haben ihn schon früh mit politischen Themen konfrontiert. Er erklärt, dass er schon als Kind begonnen habe, Zeitung zu lesen, und sich schon in der Grundschule für gesellschaftspolitische Themen interessiert habe.

Das ging dann irgendwann darin über, dass ich gesagt habe, dass man sich damit befasst und dass man sich Informationen holt und debattiert über Themen, aber wenn man sich eine Meinung gebildet hat, dann sollte man die auch kundtun und sich dafür einsetzen. So habe ich dann angefangen, mich mehr zu engagieren. (HELGE: 202-210)

Auch LEA ist überzeugt, dass ihr eigenes politisches Engagement, vor allem aber ihr politisches Interesse, nachhaltig von ihren Eltern geprägt wurde.

$\mathrm{Ja}$, ich glaube, es liegt daran, dass meine Eltern mir ziemlich viel mitgegeben haben. Ich glaube, ich habe schon ziemlich früh angefangen, so was von Politik mitzubekommen, weil mein Vater Jurist ist und sich dadurch ziemlich viel mit dem Gesetz auseinandersetzt. (LEA: 423-426)

Die junge Schülerin verweist darauf, dass ihre Eltern ihr oft die politischen Dimensionen alltäglicher Entscheidungen aufgezeigt haben. Sie verdeutlicht das am Beispiel der Produktion von Kleidung und ihrem Konsumverhalten: 
Dann haben meine Eltern mir natürlich immer wieder verschiedene Sachen einfach beigebracht, die sich mit Politik in gewissem Maße auseinandersetzen. Zum Beispiel, als ich das erste Mal mit meinen Freunden shoppen gehen wollte, haben sie dann auch gesagt, dass manche Kleider in Bangladesch hergestellt werden. Sie haben mir gezeigt, wie die Herstellung von Kleidern in Bangladesch abläuft. Und dann habe ich gleich das Bewusstsein vor Augen bekommen, dass das, was ich kaufe, von anderen Menschen unter etwas schlechteren Bedingungen hergestellt wurde, und habe mir dreimal überlegt, ob ich es kaufe. Meine Eltern haben nach den Schritten, die ich gemacht habe, mir mehr vor Augen geführt, was die Sachen, die ich mache, bedeuten. Und ich glaube, dadurch ist es so gekommen, dass ich mich auch für Politik interessiert habe. (LEA: 432-443)

ESRA berichtet noch von einer anderen wichtigen Funktion, die Eltern politisch engagierter Kinder und Jugendlicher einnehmen können. Ihre Mutter unterstütze sie in ihrem politischen Engagement. Vor allem aber sei es ihrer Mutter immer wichtig, dass sich ESRA nicht instrumentalisieren lasse. Ihre Mutter hat ihr einen Leitsatz mitgegeben, den sie im Interview besonders hervorhebt: „Lass dich nicht lenken von dem, was die anderen denken. "(ESRA: 534) Aber auch die Freund*innen nehmen eine wichtige Funktion im Politisierungsprozess ein, vor allem für den Schritt von der Bereitschaft zur Teilhabe hin zur tatsächlichen politischen Aktion. Hier steht eine Schilderung von ESRA stellvertretend für mehrere Erfahrungsberichte. Ohne ihre Freundin hätte ESRA kaum zu der selbstverwalteten Antirassismus-Arbeitsgemeinschaft an ihrer Schule gefunden: „Als ich, ich bin durch eine Freundin auf die AG gekommen und erst mal hatte ich selber noch nie von der AG gehört, obwohl ich ja auf der Schule bin und man ja eigentlich so etwas, aber irgendwie habe ich das nicht mitbekommen. " (ESRA: 148-151) Dieser Effekt konnte auch in der Mixed-Methods-Untersuchung „Jung - politisch - aktiv?!“, einer Jugendstudie der Friedrich-Ebert-Stiftung (FES) (Gaiser u. a. 2016) aus den Jahren 2015 und 2016 nachgewiesen werden. Wolfgang Gaiser und Johanna de Rijke kommen zu dem Ergebnis: „Informelle soziale Netze, also Freundeskreise, spielen eine wichtige Rolle für politische Partizipation, jedoch vor allem dann, wenn es $[\ldots]$ relevante Freundeskreise sind, hier also: politisch relevante, aktive Freunde. " (Gaiser/Rijke 2016, 68 - Hervorhebung im Original). Auch im qualitativen Teil der FES-Studie kommen Katharina Sandbrink und Jacob Steinwede (2016, 98-102) zu ähnlichen Befunden und verweisen darauf, dass vor allem das soziale Umfeld die Politisierung und das tatsächliche politische Engagement junger Menschen beeinflusst.

Neben dem Einfluss des sozialen Nahraums identifizierten die Autor*innen der FES-Jugendstudie weitere wichtige Faktoren wie das Geschlecht, das Alter und den Bildungsstand der politisch aktiven Jugendlichen. In Bezug auf den Faktor 
Geschlecht stellt Hannah Grohe fest, dass sich bei der Bereitschaft zu politischer Partizipation kaum Unterschiede zwischen den Geschlechtern feststellen lassen. ,Zwischen den einzelnen Partizipationsformen variieren diese allerdings erheblich." (Grohe 2016, 76) Junge Frauen neigen eher zu nicht-institutionalisierten Partizipationsformen wie Demonstrationen, Flashmobs und Unterschriftenaktionen. Junge Männer engagieren sich eher in konventionellen Partizipationsformen wie der Mitwirkung in Jugendgremien, Parteien oder Gewerkschaften (Grohe 2016, 76). Dieser Eindruck lässt sich auch aus den Erfahrungen der Erschließung des Forschungsfeldes für die vorliegende Untersuchung bestätigen. Insgesamt waren deutlich mehr junge Frauen in den selbstbestimmten und selbstorganisierten Gruppen aktiv. Insgesamt zeigt sich, dass Jugendliche mit zunehmendem Alter politisch aktiver werden (Gaiser/Rijke 2016, 59). Bildung hat insgesamt den größten Einfluss auf die Partizipationsbereitschaft und das tatsächliche politische Engagement junger Menschen. Dirk Lange, Holger Onken und Tobias Korn konnten schon 2013 in ihrer Studie im Auftrag der Friedrich-Ebert-Stiftung „Politikunterricht im Fokus. Politische Bildung und Partizipation von Jugendlichen“ den hohen Stellwert von Elternhaus, Bildung und explizit politischer Bildung für die Ausprägung des politischen Interesses und die Partizipationsbereitschaft nachweisen (Lange/Onken/Korn 2013). Je höher der (angestrebte) Bildungsabschluss, desto höher die Bereitschaft sich politisch $\mathrm{zu}$ engagieren. Besonders deutlich werden diese Unterschiede im Bereich der unkonventionellen Partizipation (Gaiser/Rijke 2016, 60 f.). Auch hier können die Befunde der Studien durch die Erfahrungen der Feldforschung für die vorliegende Untersuchung bestätigt werden. Wie in Abschnitt 3.2 ,Forschungssample“ beschrieben, setzt sich auch die Gruppe der interviewten Jugendlichen vorwiegend aus Schüler*innen zusammen, die aus einem akademisch geprägten Elternhaus kommen und das Abitur anstreben. Bestätigt werden diese Erkenntnisse auch durch eine Untersuchung zur Jugendprotestbewegung „Fridays for Future“. Moritz Sommer, Dieter Rucht, Sebastian Haunss und Sabrina Zajak (Sommer u. a. 2019, 13) kommen zu dem Ergebnis, dass mit 87,2 \% die große Mehrheit der Teilnehmenden und Aktiven der Bewegung entweder das Abitur als nächsten Bildungsabschluss anstreben $(55,1 \%)$ oder bereits studieren $(32,1 \%)$.

Neben den Faktoren wie Geschlecht, Alter, Bildungsstand und soziale Netzwerke konnte aus den Interviews ein weiterer Faktor identifiziert werden, der in Studien bislang kaum berücksichtigt wird: Politische Sozialisation durch den Wohnort. Beispielhaft wird dies im Folgenden an den Schilderungen von RONJA deutlich, die für sich die Großstadt als einen relevanten Sozialisationsort beschreibt und die Sensibilisierung für diversitätssensible Sprache aus der Stadt 
mit in den ländlichen Raum getragen hat, als sie mit ihrer Familie dorthin gezogen ist.

Also, meine Eltern sind sehr offen und tolerant. Mein Vater hat, also, meine Mutter ist Künstlerin und wir kommen ursprünglich aus Hamburg. Wir sind hierhergezogen. Wir kommen aus dem Schanzen-Viertel und sind halt super liberal und offen für alles. Wir wurden, das ist auch ganz spannend, direkt damit konfrontiert. Also, ich bin hier an das Gymnasium gewechselt und wurde direkt mit ganz, viel Politischem konfrontiert. Das hier auf einmal Leute „Neger" sagen, wobei ich in Hamburg niemals gedacht hätte, dass es jemals jemand aussprechen würde, weil es voll das verbotene Wort war, wurde hier einfach direkt verwendet oder andere Sachen. (RONJA: 789-799)

Neben den bis hier beschriebenen Aspekten sind Schlüsselerlebnisse als motivationale Faktoren hervorzuheben. So beschreibt LEA beispielsweise, dass die Einführung einer Einstiegsklasse für Schüler*innen mit Fluchterfahrung an ihrer Schule für sie einen solchen Moment dargestellte habe.

\begin{abstract}
Aber dann, als ich irgendwie am Anfang von der 8. Klasse war, haben wir halt eine Klasse an die Schule bekommen, die speziell für Kinder aus Familien mit Fluchterfahrung war, und da dachte ich irgendwie so, eigentlich ist es totaler Quatsch und eigentlich sollte ich mich einbringen. Weil, es ist voll wichtig, dass ich es mache und es wäre ja total doof, wenn niemand da mitmachen würde. Und wenn alle so denken würden wie ich und sich keiner traut, dann würde da gar keiner sein. Und deswegen habe ich dann irgendwie, gerade als wir die Einstiegsklassen bekommen haben und wieder mehr Unterstützung gebraucht wurde, habe ich es dann irgendwie ausprobiert und wollte erst mal nur so gucken. (LEA: 390-398)
\end{abstract}

Auch für den Politisierungsprozess von SOPHIE war das Thema Flucht und Migration bedeutsam. Mit der versuchten Abschiebung eines Mitschülers war es ein ganz konkreter Anlass, der letztlich zu ihrer Politisierung und auch zur Gründung ihrer lokalen Jugendbewegung geführt hat.

Und ich bin, wie ich dazu gekommen bin, ist hauptsächlich auch wieder über dieses Thema Flüchtlinge oder Geflüchtete, weil bei uns an der Schule einer abgeschoben werden sollte und ja, so hat sich das Ganze dann irgendwie entwickelt. Und da habe ich mir gedacht: Okay, man muss da wirklich etwas machen. Es geht ja nicht, dass den Leuten einfach ihre Zukunft, ihre Perspektiven weggenommen werden und ja, so jeder hat das Recht auf Bildung und du kannst nicht einfach die Leute aus der Schule abschieben oder ganz generell keine Leute abschieben (lacht verunsichert). Das war einfach so dieses einschneidende Erlebnis, wo man dann gemerkt hat: okay, scheiße, man muss doch was machen. (SOPHIE: 139-148) 
Auslöser dafür, dass aus politischer Partizipationsbereitschaft auch tatsächlich politisches Handeln erwuchs, war ein konkretes Ereignis. Angetrieben wird sie aber, das macht sie später im Verlauf des Interviews noch einmal deutlich, von einem „Bedürfnis nach Gerechtigkeit“ (SOPHIE: 431-432). Diese grundsätzliche Haltung treibt auch FRANZISKA an. Denn auch für die Berufsschülerin bedurfte es letztlich eines konkreten Ereignisses, um sich aktiv politisch einzubringen. Für FRANZISKA war der erste größere Bildungsstreik im 21. Jahrhundert der entscheidende Schlüsselmoment. Die Bildungsproteste in den Jahren 2008 und 2009 haben sie politisiert. Sie fand so schon vor über zehn Jahren zum politischen Engagement.

Da gab es diese Bildungsstreikbewegung von Schülern bundesweit und auch Studenten, die sich dafür eingesetzt haben, für mehr gleiche Chancen in der Bildung eigentlich. Also, da ging es um den Abbau von diesem dreigliedrigen Schulsystem, um das Abschaffen von den sogenannten Kopfnoten, wo dann bewertet werden sollte, wie man sich zum Beispiel benimmt oder so, gegen das G8-Abitur, also das beschleunigte Abitur in nur acht Jahren Gymnasium, das einfach nachgewiesenermaßen Leute benachteiligt hat, die eher aus einem bildungsferneren Haushalt gekommen sind. Gegen Studiengebühren oder jegliche Bildungsgebühren, für so Sachen wie kostenfreies Mittagsessen in der Schule. Das waren alles Sachen, die ich gut fand und deswegen habe ich zu dieser Bewegung gefunden. (FRANZISKA: 448-458)

Neben den Schlüsselmomenten, die offenbar nachhaltig Einfluss auf den Übergang von politischer Partizipationsbereitschaft und tatsächlichem Handeln haben können, ist auch die persönliche Betroffenheit für Jugendliche relevant. ESRA, eine junge Gymnasiastin, die in Deutschland geboren ist und deren Familie eine Migrationsgeschichte hat, berichtet davon, dass ihr Engagement gegen Rassismus vor allem darauf beruhe, dass sie es nicht mehr habe hinnehmen wollen, dass Menschen mit Migrationsbiografie mit Vorurteilen konfrontiert würden:

Oder dass man, nur weil ich jetzt Libanesin bin, mir irgendwie sagt, dass ich nur bei Aldi einkaufen gehe oder so. Diese Vorurteile gegenüber Leuten mit Migrationshintergrund vor allem und auch gegen einfach Rassismus und Diskriminierung, da wollte ich mich auf jeden Fall dagegen einsetzen, dass das wenigstens nicht in unserer Schule stattfindet und ich mich in meiner Schule frei fühlen kann. (ESRA: 181-186)

In der selbstorganisierten Arbeitsgemeinschaft gegen Rassismus hat ESRA einen Freiraum gefunden, der es ihr ermöglicht, sich politisch zu engagieren und dabei gleichzeitig nicht in Rechtfertigungszwänge zu geraten. „Und mir ist es, meinen Freunden ist es scheiß egal, wenn ich mir jetzt meine Haare kurz schneide oder nicht, weil es ihnen egal ist, wie ich aussehe. Weil es ihnen egal ist, wo ich herkomme. 
Weil es ihnen egal ist, wie groß oder wie klein ich bin. " (ESRA: 326-330) Die Schülerin empfindet die Arbeitsgemeinschaft als einen politischen Schutzraum, weil sich die Gruppe durch eine grundsätzliche Offenheit auszeichnet. Dadurch hat sich ein „,komplett neue[r] Freundeskreis “ (ESRA: 332) gebildet.

Unter Berücksichtigung der Perspektive eines nachhaltigen politischen Engagements ist der Faktor der Gruppendynamik von besonderer Bedeutung. Nahezu alle Schüler*innen betonen, dass der Charakter der Gruppe, der Umgang untereinander entscheidend dafür sei, dass sie sich längerfristig auf diesem Weg politisch einbringen. BEN gibt diese Gruppendynamik das Gefühl, nicht allein zu sein mit dem Bedürfnis, etwas ändern zu wollen: „Aber sich auch mit denen auszutauschen, auch dieses Gefühl zu haben, man ist irgendwie nicht ganz alleine mit dem, was man denkt. “(BEN: 243-244) Auch für LAURENZ sind die sozialen Beziehungen, die er im Zuge seines politischen Engagements aufgebaut hat, wichtig.

Soziale Kontakte spielen dann natürlich auch wieder eine Rolle. Man arbeitet ja im Kollektiv, man arbeitet nicht einzeln. Es ist einfach insgesamt ein sehr solidarisches Miteinander, was dann, zumindest für mich, erlebbar wird. Im Team zu arbeiten und all das, das bekräftigt mich ungemein. Also, es sind einfach, oft von allen Ecken und Enden, positive Sachen, die da zusammenkommen. (LAURENZ: 298-303)

Abschließend ist noch ein Faktor zu berücksichtigen, der in wissenschaftlichen Untersuchungen zu politischer Partizipation eher eine untergeordnete Rolle spielt, der den interviewten Schüler*innen aber besonders wichtig erscheint: Politisch zu handeln, Einfluss zu nehmen, politisch aktiv zu sein mache Spaß, betonen mehrere befragte Jugendliche (u. a. MICHAEL: 156-159; FRANZIKSA: 493494; JOHANNES: 457-463). Und sie verweisen darauf, dass es ihnen wichtig sei, dies auch zu vermitteln.

Nachdem dieses Kapitel einen Überblick über die verschiedenen motivationalen Faktoren für das politische Engagement der Jugendlichen ermöglichte, soll im Folgenden in die Rekonstruktion der politischen Bildungserfahrungen der Jugendlichen eingestiegen werden. Zunächst werden dabei die Schilderungen der Schüler*innen unter Berücksichtigung der Entwicklung von Analysefähigkeit und politischer Orientierung analysiert. 


\subsection{Rekonstruktionen politischer (Selbst)Bildungserfahrungen}

\subsubsection{Analysefähigkeit und politische Orientierung}

Sozialwissenschaftliches Analysieren und Denken sowie politische Orientierung sind wesentlicher Bestandteil didaktischer Konzeption für das politische Lernen. Analyse und Orientierung im Feld der politischen Bildung bedeuten, fachunspezifische methodische Fertigkeiten wie Lesekompetenz, Informationsrecherche und Quellenkritik zu stärken und sie zugleich mit sozialwissenschaftlichen Methoden und Begriffen zu verknüpfen. Für die politische Bildung hat die Auseinandersetzung mit Informationen dabei einen besonders hohen Stellenwert. Eine kritisch reflektierte Auseinandersetzung mit politischem Wissen und unterschiedlichen Informationsquellen sowie eine individuelle und kollektive Quellenkritik sind Grundlage für politische Urteilsbildung. Damit eng verbunden sind die Wahrnehmung des politischen Konflikts in der Sache sowie vielfältige wertegebundene Zugänge zu politischen Themen. Daher steht im Fokus der empirischen Rekonstruktion der (Selbst)Bildungserfahrungen der Jugendlichen neben der Praxis sozialwissenschaftlicher Methoden auch die Frage, inwiefern die Aussagen der Aktivist*innen auf eine kritisch reflektierte Konflikt- und Gesellschaftsanalyse schließen lassen. Dabei wird auch untersucht, inwiefern die Erfahrungen mit selbstbestimmter politischer Aktion die Verortung des Subjekts in der Welt, also das politische Bürgerbewusstsein (Lange 2005, 2008a), beeinflusst. Die im Folgenden dargestellten Ergebnisse der Feldforschung werden daher gegliedert in "Quellenarbeit und Umgang mit Informationen“ sowie „Konflikt- und Gesellschaftsanalyse“, wobei dabei immer auch berücksichtigt wird, inwiefern die Jugendlichen ihre eigene Analysefähigkeit im Kontext des politischen Engagements reflektieren.

\subsubsection{Auseinandersetzung mit Informationen und Quellenkritik}

Quellenarbeit ist für die interviewten Jugendlichen ein besonders wichtiges Thema. Immer wieder sprechen sie die Notwendigkeit einer kritisch reflektierten Quellenarbeit an. Sie beschreiben verschiedene Fertigkeiten der Informationsbeschaffung und der Quellenkritik, die sie im Verlauf ihres Engagements gelernt haben. Besonders wichtig erscheint ihnen dabei, dass die Informationen, auf denen sie ihr politisches Urteil und ihre politische Argumentation aufbauen, verschiedenen Medien entstammen. Sie formulieren auch einen hohen Anspruch an die Qualität der Quellen. Dabei ist hervorzuheben, dass sie ganz explizit auch darauf verweisen, dass sie insbesondere jene Quellen hinterfragen, die aufgrund 
der politischen Nähe zu ihrer eigenen politischen Haltung vermeintlich besonders zugänglich sind. So bezeichnen sich die interviewten Jugendlichen weitgehend als links, kritisieren aber auch linke Plattformen für ihre einseitige Berichterstattung oder die Qualität der dort aufbereiteten Informationen. Exemplarische sei hierfür eine Aussage von BEN angeführt:

\begin{abstract}
Also, es gibt halt linke Plattformen zum Beispiel auch im Internet, die darüber Berichte schreiben, wo man dann aber auch teilweise irgendwie sagen muss, dass die nicht ganz qualitativ unglaublich hochwertig nachgeforscht sind. Dann versuche ich, mich auch bei bürgerlichen Quellen zu informieren und zu gucken, ob es da Widersprüche gibt. Wenn ich die Widersprüche finde, dann irgendwie gucken, ja, keine Ahnung, finde ich da vielleicht noch andere Belege zu. Also, ich bemühe mich da schon, nicht einfach irgendwas aufzuschnappen und das dann einfach weiterzuschreien und irgendwie rumzurufen. (BEN: 380-388)
\end{abstract}

BEN begründet diesen Anspruch an Quellenarbeit für sein politisches Engagement auch damit, dass er vermeiden möchte, dass die eigene politische Arbeit an Glaubwürdigkeit verliert.

Weil ich denke, das führt am Ende zu Phänomenen, dass dir am Ende irgendwie keiner mehr glaubt. Und das möchte ich nicht. Und das versuche ich zu vermeiden, also, keine Falschinformationen zu verbreiten. (BEN: 388-390)

Den Berichten der Kinder und Jugendlichen aus den unterschiedlichsten Kontexten des selbstbestimmten und selbstorganisierten politischen Engagements ist $\mathrm{zu}$ entnehmen, dass sie im Umgang mit Informationen von ihren Erfahrungen profitieren. Sie führen vielfältige Fähigkeiten der Quellenarbeit an. Dabei wird immer wieder betont, dass die Entwicklung dieser Fähigkeiten hauptsächlich auf die politische Arbeit zurückzuführen ist. Es ist die Fülle an Informationen gemeint, mit der sie sich auseinandersetzen, aber auch die Erkenntnis über die Bedeutung einer qualitativ hochwertigen Recherche für eine überzeugende politische Argumentation. Beispielhaft hierfür ist folgendes Zitat von LAURENZ, der den Einfluss seiner politischen Bezugsgruppe auf seine Arbeit mit Informationen und Quellen beschreibt.

In der Gruppe lerne ich eben auch, wie andere Leute das machen. Wie die anderen damit umgehen. Und ich merke auch im politischen Diskurs, im zwischen-menschlichen Diskurs, die qualitativen Unterschiede an Informationen, die irgendwie mir entgegengebracht werden. Und je nachdem, was derjenige, mit dem ich dann zum Beispiel spreche, woher der diese Informationen hat. So unterscheidet sich dann eben auch die Qualität dessen, was er auch sagen kann. Und da merke ich dann auch, dass es 
ziemlich wichtig ist, dass ich auch auf gute Quellen zurückgreifen kann. Auf viele und auf gute Quellen, um auch selber überzeugend zu sein und auch sowohl für mich als auch für andere überzeugend zu sein. (LAURENZ: 451-460)

Weiter führt LAURENZ aus, dass er sich bereits vor seinem politischen Engagement häufig ein Urteil gebildet habe, dieses aber zumeist auf wenigen und eher unseriösen Quellen aufgebaute habe (LAURENZ: 470-481). Auch ESRA bestätigt den Einfluss der politischen Bezugsgruppe und des politischen Engagements auf die Wahrnehmung von Nachrichten und politischen Inhalten. „Ich achte immer mehr auf Nachrichten. Früher war mir das eigentlich relativ egal. " (ESRA: 313-315)

Ganz konkret beschreiben die interviewten Jugendlichen, wie sich die Art der Informationsbeschaffung und Reflexion durch ihr politisches Engagement verändert hat. Während zuvor Informationen zunächst in Onlinesuchmaschinen gesucht wurden und die ersten Treffer als Quelle für ausreichend eingeschätzt wurden, verweisen die Jugendlichen darauf, dass die Suche nach Informationen komplexer geworden ist und der Anspruch an die recherchierten Informationen steigt.

Mittlerweile gucke ich mir immer genau an, woher die Sache kommt, oder so, wenn ich irgendwelche Zahlen recherchiere. Wenn man im Allgemeinen recherchiert, fällt ja oft auf, dass man verschiedene Faktenlagen im Prinzip findet zum gleichen Thema. Da bin ich auf jeden Fall kritischer geworden. Und es ist mir mittlerweile auch einfach wichtiger geworden, mehr zu lesen zu einem Thema und mich nicht so schnell mit der Sache zufrieden zu geben und mich auch auszutauschen und nicht einfach irgendwas anzunehmen, als gegeben oder so. Sondern dann nochmal vielleicht mit jemand anderem zu reden, der sich vielleicht mit der Sache genauer beschäftigt hat. (FRANZISKA: 652-661)

Onlinemedien spielen dabei für die Jugendlichen eine entscheidende Rolle. Sie bewegen sich oftmals auf den Internetpräsenzen großer Nachrichtenprotale und Tageszeitungen. Sie lesen aber auch Blogs und folgen politischen Social-MediaKanälen. „Ich lese viel Zeitung, ich lese viele Blogs im Internet, auch generell viele Internetartikel auch von unabhängigen Leuten auf Social-Media-Accounts oder kleinen Websites[.] " (HELGE: 275-278)

Neben der Vielfalt an Quellen, mit denen sich die politisch aktiven Jugendlichen auseinandersetzen, betonen sie immer wieder auch die Notwendigkeit eines kritisch reflektierten Blicks auf Medien und Informationen. Hervorzuheben ist dabei die von den Interviewten betonte Wechselwirkung von politischem Interesse, politischem Wissenserwerb und politischer Aktion. 


\begin{abstract}
Also, ich brauche dafür ganz viele Informationen, auf jeden Fall. Und ich möchte ganz. viele Informationen haben. Erstmal lese ich momentan sehr viel, also, das ist jetzt auch wiedergekommen. Es hängt irgendwie in Wechselwirkung zusammen, einerseits durch das Lesen bin ich dann auch wieder zum politischen Interesse gekommen und durch das politische Interesse lese ich dann auch wieder mehr. Es bedingt sich irgendwie gegenseitig. Also, ich muss ja auch irgendwie theoretisch auf Zack sein. (LAURENZ: 418-425)
\end{abstract}

Die interviewten Jugendlichen beschreiben in ihren Erfahrungsberichten neben den weiterentwickelten Fähigkeiten zur Recherche von Informationen auch die Entwicklung neuer Kompetenzen im Umgang mit den recherchierten Informationen. So betont LAURENZ beispielsweise, dass er durch seine politische Arbeit auch das systematische Markieren von Texten verinnerlicht habe. „Es hat mich, glaube ich, in vielfältiger Weise verändert, [...] allein schon das Markieren von Texten, alleine das kann ich schon viel besser durch meine politische Arbeit." (LAURENZ: 322-326)

Quellenarbeit spielt für die Jugendlichen aber nicht nur im Kontext der individuellen Selbstbildung eine entscheidende Rolle. Sie bereiten auch Informationen für ihre Mitstreiter*innen auf. Sie organisieren Lesekreise (u. a. BEN: 288295; FRANZISKA: 507-521; LAURENZ: 393-399), in denen Texte anderer reflektiert werden und die von politisch aktiven Jugendlichen vor allem zur PeerEducation in der Gruppe genutzt werden. Die Jugendlichen verweisen darauf, dass sie sowohl die Strategien bei der Informationsrecherche als auch den kritischen Blick auf die verschiedenen Medien unter anderem von älteren oder erfahreneren Jugendlichen gelernt haben. Die Jugendlichen messen der Informationsgewinnung und dem Austausch eine besondere Bedeutung für ihren politischen Selbstbildungs- und Sozialisationsprozess bei.

Für FRANZISKA hat die Peer-Group einen besonderen Einfluss auf diesen Lernprozess. Politisierung in der politischen Aktion bedeutet für sie, sich mit Themen zu befassen, Informationen zu sammeln, sie zu reflektieren, sich ein Urteil zu bilden und dabei keinen Leistungsdruck zu spüren oder Erfolgserwartungen erfüllen zu müssen. Die Motivation, sich mit Informationen und Quellen auseinanderzusetzen, ist intrinsisch motiviert und erhält daher eine andere Bedeutung für die Jugendlichen. Die Quellenarbeit ist für FRANZISKA und für die Gruppe, in der sie sich engagiert, auch deswegen bedeutsam, weil es ihnen ein Anliegen ist, ihre Ideen, theoretischen Überlegungen und den politischen Protest weiterzuentwickeln und zu untermauern.

Die Leute, mit denen ich dann politisch aktiv war, die haben dann auch Sachen organsiert, wie zum Beispiel Lesekreise. Und das hat mir einfach total Spaß gemacht, andere 
Bildungsräume kennenzulernen, wo eben nicht vorne der Lehrer steht, der irgendwas erzählt. Dann lernt man das auswendig und kriegt irgendwann eine Prüfung zu dem Thema. Sondern wo man gemeinsam auf der Grundlage von einem Text, den man vorher liest, erst Verständnisfragen klärt und dann darüber diskutiert, was jeder darüber denkt. Und dann kann man auch wirklich einfach sagen, was ist meine persönliche Meinung. Und man tritt in den Diskurs ein und es geht nicht um irgendein Ziel, um irgendeine Note oder um irgendein Zertifikat oder so etwas, das man am Ende dafür bekommt. Das heißt, dieser unangenehme Leistungsdruck und auch Ehrgeiz fällt erstmal weg und man macht was nur für sich persönlich, um sich selber weiterzuentwickeln und um seinen Protest auch zu entwickeln anhand dieser Theorien, die man vielleicht liest. (FRANZISKA: 507-521)

Neben den Lesekreisen ist Quellenarbeit vor allem auch Teil der internen Workshops der Bezugsgruppen. Zu unterschiedlichen Themen bereiten die Jugendlichen Selbstlern-Workshops vor. So hat sich der überwiegende Teil der beforschten Jugendgruppen, die einen antirassistischen Schwerpunkt haben, beispielsweise gruppenintern mit der Nahost-Frage (u. a. LAURENZ: 551-570) befasst, aber auch Themen wie die Identitäre Bewegung oder der Klimawandel wurden in internen Workshops aufgearbeitet. Hierfür setzen sich die Jugendlichen intensiv mit Fachtexten auseinander.

Wenn wir das Gefühl haben, wir brauchen eine Gruppenposition, die natürlich dann auch wieder veränderlich ist, wo auch keiner ausgeschlossen wird, wenn er was dagegen hat, dann versuchen wir meistens, Workshops zu veranstalten. Also, das sind dann teilweise, das geht dann drei Tage lang zum Beispiel, also nicht hintereinander, sondern meistens dann etwas versetzt, wo sich dann fünf Menschen zum Beispiel dransetzen und den vorbereiten, also sich Literatur raussuchen, möglichst von beiden Seiten, um auch ein differenziertes und kritisches Bild von der Lage zu bekommen. (BEN: 306-313)

Sie diskutieren und reflektieren fremde Texte in Lesekreisen und Workshops, aber auch selbst verfasste Texte, die als Reden auf Demonstrationen oder auf ihren eigenen Seiten im Internet und den Sozialen Netzwerken wie Instagram und Facebook vorgestellt werden. Dabei legen sie vor allem Wert auf solidarische Kritik (LAURENZ: 390). Dieser Prozess führt zu einer veränderten Wahrnehmung und kritischen Würdigung von Informationen und Nachrichten. Dazu zählt, dass die Jugendlichen durch ihr politisches Engagement das Bedürfnis entwickeln eigene Wissenslücken zu schließen (BEN: 375-379). Darüber hinaus bietet das politische Engagement auch einen unmittelbaren Zugang zu anderen, den Jugendlichen bis dahin eher unbekannten Formaten politischer (Selbst)Bildung. So stellt die Berufsschülerin SOPHIE fest, dass ihr der Zugang zu komplexen Texten noch 
immer schwerfalle, sie aber durch die politische Arbeit andere Formate wie Diskussionsrunden und öffentliche Vortragsveranstaltungen $\mathrm{zu}$ schätzen gelernt habe (SOPHIE: 497-514).

Den Erfahrungsberichten der Jugendlichen ist außerdem zu entnehmen, dass ihr Engagement ein anderes Bewusstsein und eine neue Wahrnehmung für Nachrichteninhalte schafft: „Ja (...3...), ich überdenke Sachen und ich sehe politische Themen und die Nachrichten zum Beispiel auch ganz anders, als ich sie vorher gesehen habe. " (ESRA: 355-356) Die junge Schülerin ESRA führt weiter aus:

Naja, also, ich achte mehr auf kleine Details, ich achte auf die Informationen. Da ich mehr Vorwissen habe, weiß ich, worum es geht. Ich kann mich danach auch mit dem Thema auseinandersetzen. Ich kann mich danach noch mehr belesen, weil ich jetzt diese Grundinformationen habe und ich weiß, worum es geht. Das hilft mir auch viel mehr im Alltag, würde ich, also, das hilft mir, dass ich mich da hinsetzen kann und die Nachrichten auch verstehen kann, was ich früher zum Beispiel gar nicht verstanden hab. (ESRA: 360-366)

Das höhere Maß an Fachlichkeit ermöglicht ESRA, politische Themen und Konflikte in ihrer Vielfalt zu verstehen und einzuordnen. Sie beschreibt ihre analytischen Fähigkeiten damit als ausgereifter im Vergleich zu der Zeit vor ihrem Engagement in der Antirassismus-AG. Besonders hervorzuheben ist, dass die Jugendlichen nicht nur davon berichten, dass sie sich intensiver mit Nachrichten befassen, sondern Inhalte, mit denen sie konfrontiert werden, auch eher reflektieren.

Wenn man lernt, das zu reflektieren, was andere sagen und was man selbst sagt, was man halt zum Beispiel in der AG oder in einer Diskussion oder so lernt, dann ist es auch im echten Leben leichter, so Sachen zu reflektieren. [...] Und das lernt man halt durch sich engagieren und selbst was machen, was man dann hinterher reflektieren kann und überlegen kann, was könnte man besser machen. (LEA: 637-644)

Reflexion der eigenen Lernfähigkeit ist für LEA, anders als im Unterricht, nicht mit Zwang verbunden. Diese mit dem Unterricht verbundene konstruierte Reflexion sei weniger motivierend, meint LEA.

Doch auch die Schule empfinden die Jugendlichen als einen wertvollen Erfahrungsraum zur Stärkung des Umgangs mit Quellen. So haben RONJA und ihre Mitstreiter*innen für ihre politischen Projekte immer wieder im Internet recherchiert, sie haben Quellen wie die Süddeutsche Zeitung genutzt, aber auch Daten des statistischen Bundesamtes und Informationen der Bundesregierung eingebunden. Für diese komplexen Recherchearbeiten erhielten sie bei 
Bedarf auch Unterstützung von einer Lehrkraft (RONJA: 307-329). Ganz konkret beschreibt RONJA diese offene Kooperation an einem Projekt, in dem sie und ihre Bezugsgruppe an ihrer Schule über Vorurteile aufgeklärt haben:

Ich fand es ein bisschen schwierig an manchen Stellen, die Quellen wirklich dann so zu treffen, dass man Vorurteile wirklich dann auch widerlegen kann. Aber da lernt man ja schon noch, wie man da rankommt. Und da haben wir zum Beispiel Herrn Hansen für den Part noch einmal angerufen, nach Zahlen aus der Stadt, weil er in der Stadtverwaltung aktiv ist, gefragt, ob man da an Zahlen rankommt. (RONJA: 350-357)

Auch für ESRA ist die pädagogische Begleitung durch Lehrkräfte wichtig. Sie verweist darauf, dass auch die begleitenden Lehrkräfte der Arbeitsgemeinschaft Texte als Diskussionsgrundlage zur Verfügung stellen. ESRA ist froh, dass es unterschiedliche Formen der Auseinandersetzung mit Informationen und Quellen in der Arbeitsgemeinschaft gibt.

Wenn nicht, wenn wir gerade keine Themen haben und wir in der AG echt nicht wissen, was wir machen sollen, dann kommen die Lehrer oft mit irgendwelchen Informationstexten, zum Beispiel über Gendern oder was war das noch, ja, über den Türkei-Deal zum Beispiel, all so etwas, damit wir erst mal Grundlagen bekommen und uns dazu etwas durchlesen. Und danach fragt zum Beispiel eine Lehrerin: Okay, was haltet ihr davon? Oder: Habt ihr dazu etwas anzumerken? Und dann fängt es immer an mit einer Diskussion. Und das finde ich auch das Schöne, das man sagt, dass wir jetzt, falls es keine Vorfälle gibt, dass wir uns auch einfach politisch auch ein bisschen weiter, ja, unser Wissen ein bisschen ausbreiten können. (ESRA: 294-303)

Neben der offenen Kooperation mit Lehrkräften bietet vor allem die PeerEducation in der Entwicklung von analytischen Grundkompetenzen wie der Quellenarbeit ein großes Potenzial und stellt dabei auch einen motivationalen Faktor dar. Politische Aktionen in Selbstorganisation bieten hierfür vielfältige Lernanlässe. ESRA, die sich in einer selbstorganisierten rassismuskritischen Arbeitsgemeinschaft an ihrer Schule engagiert, verweist auf den Wert der altersheterogenen Zusammensetzung der Arbeitsgemeinschaft:

Und dass wir durch die ganzen Diskussionen, was früher zum Beispiel sehr schön war, dass es viele Leute von der Oberstufe gab, die uns viel beibringen konnten. Ich weiß jetzt viel, viel mehr als vorher, weil man einfach sagt: Okay, ich kann zwar darüber diskutieren, aber nur über das Wissen, das ich durch die Nachrichten kennengelernt habe. Und das ist ja oft nicht so viel und dass uns dann die aus der Oberstufe auch was erzählen oder erklären. Das gefällt mir am meisten. (ESRA: 303-310) 
Die Recherche- und Quellenarbeit und das gemeinsame Diskutieren politischer Themen ist ein immer wiederkehrendes Element der politischen Bildungserfahrung in der politischen Aktion. Es dient dazu, sich mit Problemlagen auseinanderzusetzen und neue Handlungsfelder zu erschließen. Die kritische Auseinandersetzung mit Informationen ist aber auch Teil einer komplexen Konfliktund Gesellschaftsanalyse, die einen ebenso bedeutsamen Teil der politischen Arbeit der interviewten Jugendlichen ausmacht.

\subsubsection{Konflikt- und Gesellschaftsanalyse}

In der Politikdidaktik wird immer wieder diskutiert, inwiefern politische Aktionen als Lernanlässe den Ansprüchen politischer Bildung gerecht werden können. Dabei steht die These im Fokus, dass politische Aktion immer auch mit einer abgeschlossenen Meinungsbildung und primär mit dem Verfolgen politischer Ziele einhergeht (siehe hierzu auch Abschnitt 2.3.2 „Kontroverse: Partizipation als Ziel politischer Bildung?"). Den Interviews mit den politisch engagierten Jugendlichen ist zu entnehmen, dass sich dies in Bezug auf die beforschten Personen und politischen Gruppen nicht bestätigen lässt. Es besteht vielmehr eine Offenheit für neue Themen und der Wunsch nach einer diversifizierten Argumentation.

Für die Auswahl der Themen, die in den politischen Gruppierungen aufgegriffen werden, ist ein entscheidendes Kriterium der konflikthafte Charakter. HELGE beschreibt beispielsweise, wie wichtig es ihm und seinen Mitstreiter*innen sei, die politische Kontroverse und den politischen Konflikt zu analysieren und in ihrer Arbeit auch abzubilden.

Der Aufwand ist in dem Sinne der, dass ich mir bestimmte Themen raussuche, die vor allem momentan in der Gegenwart sehr kontrovers angesehen werden oder eben nicht kontrovers und sich wenig Leute damit auseinandersetzen und man aber der Meinung ist, es sollte mehr besprochen werden. (HELGE: 406-409)

HELGE und seine Mitstreiter*innen, die gemeinsam eine politische InstagramSeite verwalten, versuchen immer aktuelle gesellschaftspolitisch relevante Themen aufzugreifen. Sie analysieren dafür, welche Themen aktuell diskutiert werden oder welche möglicherweise mehr Aufmerksamkeit bedürfen.

Meistens ist es so: Der alte Text ist fertig und dann überlegt man, was wird momentan sehr stark in der Gesellschaft diskutiert oder was ist eben nicht so wichtig, sollte aber mehr diskutiert werden. Dann suchen wir uns zusammen ein Thema raus. Dann werden Vorschläge gemacht, dann diskutieren wir darüber und suchen uns ein Thema raus. (HELGE: 433-437) 
Auch der Schüler*innen-Initiative von RONJA ist es wichtig, gesellschaftspolitische und schulpolitische Konflikte $\mathrm{zu}$ analysieren und in ihren Aktionen $\mathrm{zu}$ berücksichtigen (RONJA: 442-450). Die politische Arbeit in den selbstverwalteten Arbeitsgemeinschaften führt auch dazu, dass die Jugendlichen für Konflikte sensibilisiert werden.

Ich hätte gar nicht gedacht, dass es so viele Vorfälle gibt, dass wir sagen: Okay, wir müssen jetzt wirklich darüber reden. Aber als ich dann darauf geachtet habe, weil ich in der AG bin, habe ich ein bisschen auf mein Umfeld geachtet und habe gemerkt, dass in meinem sozialen Umfeld viel so etwas passiert, was vielleicht auch so indirekt passiert und sich selbst, also, die Meinungen selbst innerhalb der Klasse spalten, sogar bei den Freunden spalten. (ESRA: 212-218)

Diese Sensibilisierung für politische Konflikte und ihre Wirkkraft auf das soziale Umfeld scheint nachhaltig das eigene politische Bewusstsein zu prägen und damit auch die Bereitschaft und Offenheit für einen proaktiven Umgang mit politischen Konflikten.

Auch im außerschulischen politischen Engagement liegt den Jugendlichen viel an einer ihren politischen Aktionen vorangestellten Konflikt- und Gesellschaftsanalyse. Die Themenfelder, die dabei bearbeitet werden, sind vielfältig. „Es werden Texte zu gewissen Themen ausgearbeitet, ob es jetzt zur Frauenfrage, also zu Sexismus, ob es zu Rassismus ist, Diskriminierung oder eben einer Häuserreihe, die von einem Investor aufgekauft werden soll. “ (LAURENZ: 258-261) Eine Konfliktanalyse ist dabei immer Ausgangspunkt der politischen Arbeit, wobei die Jugendlichen ein durchaus differenziertes Verständnis vom politischen Konflikt haben.

Also, Konflikt ist ja auch wieder etwas ziemlich Weitreichendes. Es gibt ja sehr viele Konflikte. Es gibt Interessenkonflikte, es gibt politische Konflikte, es gibt ökonomische Konflikte und all das. (LAURENZ: 486-488)

LAURENZ, der sich in einer autonomen Jugendbewegung engagiert, beschreibt dies am Beispiel des Themas Flucht und Asyl wie folgt:

Der Konflikt zwischen Flüchtlingspolitik oder den Erwartungen an die Flüchtlingspolitik und der Realität, der Konflikt zwischen letztendlich den Flüchtlingen und der Politik an sich, Konflikte zwischen ökonomischer Realität und den Interessen derjenigen, die die Entscheidungen tragen - all das fließt da mit ein. (LAURENZ: 490-495) 
Unabhängig vom Raum des politischen Engagements, ob in der Schule, außerhalb des Schulgebäudes oder gar in digitalen Handlungsfeldern wie Sozialen Netzwerken, die Analyse und Aufbereitung von Konflikten scheint Triebfeder des politischen Engagements der interviewten jungen Menschen zu sein. Gesellschaftsanalyse und politische Aktion scheinen dabei eine wechselseitige Motivation darzustellen. So beschreibt MICHAEL einen viel bewussteren Umgang mit eigenen und fremden Rassismus-Erfahrungen. Die Vorbereitung und Umsetzung einer Schüler*innen-Demonstration gegen Rassismus hat ihn für das Thema weiter sensibilisiert. Ausgangspunkt für sein politisches Engagement war das Ergebnis einer kritischen Auseinandersetzung mit Rassismus. Daraus folgte der Wunsch als Schulgemeinschaft, angetrieben von den Schüler*innen, ein Zeichen zu setzen. Die politische Aktion selbst aber sensibilisierte MICHAEL noch einmal mehr für eigene rassistische Verhaltensweisen. Sie regte damit einen weitergehenden kritischen Denkprozess an.

Ich denke, dass die Demo in mir selbst ziemlich viel sogar verändert hat. Vorher hat man mal in den Klassen auch manchmal wirklich rassistische Witze gemacht, aber jetzt nach der Demo sehe ich das komplett anders. Und wenn Leute das machen, spreche ich die an und sag: Hey, muss das sein? Oder ich frage sie, was sie damit bezwecken wollen. Also, es hat in mir ziemlich viel verändert, einfach, weil ich mir vorher nie so Gedanken darüber gemacht habe. (MICHAEL: 296-301)

Die überwiegende Mehrheit der interviewten Jugendlichen verweist explizit auf den Wert des politischen Konflikts als Grundlage für die Entwicklung ihres politischen Urteils. Die kritische Auseinandersetzung mit den bestehenden Verhältnissen ist für die Jugendlichen immer auch verbunden mit einer Analyse bestehender Macht- und Herrschaftsstrukturen und der damit verbundenen Konflikte. Der politische Streit wird als notwendig für Meinungsbildungsprozesse begriffen.

Ich denke aber, dass Konflikte nicht unbedingt sehr negativ sein müssen. Also, sie sind natürlich negativ konnotiert, das ist klar, aber wenn ich mit jemandem eine politische Diskussion führe, ist das ja auch auf die ein oder andere Weise ein Konflikt. Und das finde ich sehr wichtig, weil wenn ich niemals mit jemandem diskutiert hätte, der nicht meine politische Meinung teilt, dann hätte sich auch meine Meinung überhaupt nicht entwickelt. Oder nur in nicht zufriedenstellendem Maße, aus meiner jetzigen Sicht. (LAURENZ: 515-522)

Die Jugendlichen führen die positive Besetzung des Begriffs Konflikt und ihre eigene Sensibilisierung für gesellschaftspolitische Diskurse in erster Linie auf ihr eigenes politisches Engagement zurück. Analytische Reflexionsprozesse, auch 
die Aufarbeitung gruppeninterner Konflikte, seien freiwillig. Und was sie dabei lernen, könnten sie auch auf andere Kontexte übertragen.

Und im Unterricht ist es meistens so, da muss man ja auch manchmal Gruppenarbeit oder so reflektieren. Aber da ist es meistens so als Zwang dargestellt und man soll aufschreiben, was gut war und was schlecht war. Aber wenn man sich irgendwie selbst mehr damit befasst und es nicht als Zwang vorgegeben ist, hat das so viel mehr Effekt. Weil so wie es im Moment ist, ist es ja in der Schule mehr so als Zwang vorgegeben, dass man manchmal so reflektieren muss. Aber dann wird es erstens von vielen einfach nicht so ernst genommen, und zweitens hat man dann, glaube ich, nicht so den großen Lerneffekt. Und deswegen ist es einfach viel wichtiger, außerhalb des Unterrichts zu lernen und selbst zu reflektieren. Wenn man sich zum Beispiel mit Freunden streitet oder so etwas, ist es auch immer gut etwas zu reflektieren. Und das lernt man halt durch sich engagieren und selbst was zu machen, was man hinterher reflektieren kann und überlegen kann, was ich besser machen könnte. (LEA: 645-657)

Diese besonderen Rahmenbedingungen für politische Selbstbildungsprozesse ermöglichen es, komplexe politische Zusammenhänge zu erfassen. „Also, ich erfasse jetzt auch viel mehr die gesamten gesellschaftlichen Kräfte, die irgendwie am Wirken sind“. (LAURENZ: 327-330) Davon ist LAURENZ überzeugt und ergänzt: „Ich bin der Meinung, dass ich jetzt irgendwie viel bessere Analysefertigkeiten der gesamten gesellschaftlichen Situation habe. “ (LAURENZ: 327-330) Sein Textverständnis, seine Lesegeschwindigkeit und der Zugang zu politischen Texten habe sich durch sein Engagement deutlich verbessert. Er führt das darauf zurück, dass sein politisches Engagement sein Interesse an politischen Themen verstärkt habe (LAURENZ 350-370).

Die hier zusammengefassten Erkenntnisse konzentrieren sich auf analytische Elemente der Bildungserfahrungen. Im Mittelpunkt steht dabei, wie die interviewten Jugendlichen politische Konflikte wahrnehmen und welche Rolle die Analyse gesellschaftlicher Verhältnisse in ihrer politischen Arbeit einnimmt. Für diesen Teil der Rekonstruktion von Bildungserfahrungen stehen dementsprechend eher theoretische Dimensionen der Konfliktfähigkeit im Fokus - die Konfliktanalyse. Erfahrungen im Sinne einer Stärkung realer Konfliktfähigkeit werden durch die Analyse von Aussagen der Schüler*innen rekonstruiert, die im weiteren Verlauf der Arbeit vorgestellt werden (siehe Abschnitt: 4.3.4 „Partizipationserfahrungen und Konfliktfähigkeit“"). Zunächst werden aber die Aussagen der Jugendlichen daraufhin analysiert, welchen Einfluss ihr politisches Engagement auf die Fähigkeit zur Perspektivenwahrnehmung hat. 


\subsubsection{Perspektivenwahrnehmung}

Die Befähigung zur Perspektivenwahrnehmung und die offene, aber auch kritische Auseinandersetzung mit den pluralen politischen Positionen ist ein Wesensmerkmal politischer Bildungsprozesse. Es könnte die Gefahr bestehen, dass im Kontext politischer Aktion nur unzureichend Raum und Offenheit für die Wahrnehmung vielfältiger Perspektiven besteht (Reinhardt 2014, 278). Entgegen der durchaus begründeten These, dass in der politischen Aktion eine Engführung auf die eigene politische Haltung und Position bestünde, konnten - ohne dass die Perspektivenwahrnehmung expliziter Bestandteil des Interviewleitfadens war - in zehn der 13 Interviews unmittelbare Fundstellen identifiziert werden, die darauf schließen lassen, dass Perspektivenwahrnehmung einen hohen Stellenwert im (Selbst)Bildungsprozess der Jugendlichen einnimmt. Die Jugendlichen betonen wiederholt den Wert eines breiten Meinungsspektrums. Sie begründen dies damit, dass die Wahrnehmung unterschiedlicher Perspektiven und ihre Reflexion Voraussetzungen für die Bildung des eigenen politischen Urteils seien. Stellvertretend für diese Beobachtung steht die folgende Aussage von HELGE:

[I]ch glaube, es ist sehr wichtig, dass es verschiedene Meinungen gibt in der Gesellschaft. Und wenn man Leute dazu anregt, über etwas nachzudenken, und wenn sie darüber nachdenken, vielleicht auch zu sagen, was sie dazu denken, dann schafft das einen Freiraum für viele unterschiedliche Meinungen. Und das ist für die Bildung der eigenen Meinung sehr gut. Man würde niemals Alternativen finden oder Probleme so gut angehen können, wenn alle dieselbe Meinung hätten. Sondern wenn man unterschiedliche Meinungen hört und sich mit vielen verschiedenen, am besten so vielen Meinungen wie möglich auseinandersetzt, dann sieht man die Dinge auch ganz anders und lernt demnach auch sehr viel mehr. (HELGE: 349-358)

Die Jugendlichen sind davon überzeugt, dass die eigene Meinungsbildung nachhaltig von Meinungspluralität und unterschiedlichen Perspektiven profitiere. Notwendig sei dafür ein politischer Diskurs. So spricht HELGE bereits am Beginn des Interviews an, dass man mehr erreichen würde, wenn man sich mit möglichst vielen Personen und ihren Positionen auseinandersetze, ,weil man sich dann viel mehr Meinungen einholen kann und sich auch selber eine viel konstruktivere Meinung bilden kann " (HELGE: 49-51).

Dieses Grundverständnis gilt sowohl für die interviewten Jugendlichen in den schulischen als auch in den außerschulischen Gruppen. So betonen BEN und LAURENZ, die beide in außerschulischen Jugendgruppen aktiv sind, dass es in ihren Gruppen keine festgeschriebenen politischen Ansichten gibt (BEN: 
215-216) und sich die Gruppen als offen verstehen und damit im Prinzip allen ermöglichen wollen mitzuwirken:

Erstmal muss ich ja nun auch sagen, dass wir eben eine offene Gruppe sind. Das bedeutet, dass jeder daran teilnehmen kann und wir uns auch wünschen, dass jeder daran teilnimmt. Und daher muss es möglich sein, dass jeder individuell für sich entscheiden kann, was er denn verantworten kann und was er auch tatsächlich kann. (LAURENZ: 680-684)

Wenngleich die Jugendlichen den Wert von Meinungspluralität betonen, so zeigt sich dennoch in den Interviews, vor allem bei jenen Aktivist*innen, die innerhalb der Schule keine (Frei)Räume für politische Aktionen vorgefunden und daher ihr politisches Handlungsfeld außerhalb der Schule etabliert haben, dass es teilweise an der Offenheit für andere Perspektiven mangelt. Die interviewten Jugendlichen berichten davon, dass sie in ihren Bezugsgruppen immer wieder das Gefühl haben, dass der Wunsch nach Meinungspluralität und die Bereitschaft zu Perspektivenwahrnehmung kein Selbstverständnis sei. So erklärt SOPHIE, eine Berufsschülerin, die sich bei einer antirassistischen Jugendbewegung engagiert, dass es ihr wichtig sei, die Perspektiven anderer zu verstehen, sie aber gleichzeitig überzeugt sei, dass viele in ihrem Umfeld und der politischen Jugendgruppe dazu nicht bereit seien und sich für die Motive anderer politischer Positionen nur bedingt interessierten (SOPHIE: 316-332).

Ein besonderes Potential offenbaren die Interviews mit Jugendlichen, die innerhalb der Schule politische Aktionsgruppen gegründet haben. KATJA, die sowohl Erfahrung in außerschulischen autonomen Jugendprotestbewegungen gesammelt hat als auch in einer selbstorganisierten Arbeitsgemeinschaft in der Schule aktiv ist, beschreibt den Wert schulischer politischer Aktion am Beispiel der Heterogenität bezüglich politischer Positionen und des Aktionskonsenses. Zunächst sah sie das skeptisch, im Laufe der Zeit hat sie es aber als eine Stärke der Arbeitsgemeinschaft empfunden.

Ja, gerade weil die Meinungen so verschieden waren über Politik und über das, was wir eigentlich wollen, weil sonst arbeite ich irgendwie mit Leuten zusammen, die zwar nicht $100 \%$ aber größtenteils alles so ähnlich sehen wie ich. Und es gibt natürlich, man diskutiert auch mal stundenlang über, keine Ahnung Israel und Palästina, aber der Weg, wie irgendwas erreicht werden soll, ist halbwegs ähnlich. Und da sucht man sich auch irgendwie die Leute, mit denen man da konform geht und gut was machen kann. Und jetzt in der Schule ist es halt so, dass alle, die Bock haben, mitmachen. Und da gibt es halt total krasse Unterschiede. Es war total interessant überhaupt mit den Leuten darüber zu reden, was sie so wollen, aber auch zu merken, dass man trotzdem total gut zusammenarbeiten kann und zwar andere Projekte rauskommen, als wenn es 
mit Leuten ist, mit denen man eher die Meinung teilt, aber es trotzdem total interessant war und irgendwie cool. (KATJA: 396-408)

Insgesamt zeigt sich, dass, wenngleich den politischen Gegner*innen möglicherweise weniger moralische Integrität zugestanden wird, sie als relevante politische Akteur*innen verstanden werden, mit deren Positionen es sich auseinanderzusetzen gilt.

[S]elbstverständlich ist die FDP auch eine politische Organisation, aber selbstverständlich sind ihre Ziele nicht, eine solidarische Gesellschaft zu erreichen. Das ist vielleicht mein persönliches politisches Augenmaß, das was mir wichtig ist und was ich als meinen politischen Kampf oder so etwas verstehe, aber selbstverständlich ist jeder politisch, der irgendeine Vision oder so für die Gesellschaft verfolgt oder jeder, der eben ein Akteur ist in Gestaltungsprozessen. (FRANZISKA: 1042-1063)

Dieses Zitat zeigt auf, dass die Jugendlichen auch politisch Andersdenkende wahrnehmen und ihre Positionen als politisch legitim bewerten, wenngleich sie diese nicht teilen. Sie übernehmen die Perspektiven nicht, nehmen sie aber wahr.

Von besonderer Bedeutung für die vorliegende Untersuchung ist die Frage, welchen Einfluss das individuelle politische Engagement auf die Fähigkeit hat, sich in andere Personen hineinzuversetzen, Offenheit für den politischen Diskurs und Meinungspluralität zu entwickeln und politische Argumentationsmuster politisch Andersdenkender zu verstehen.

Um dieser Frage nachzugehen, wurden Aussagen in die Kategorie „Einfluss des politischen Engagements auf die Fähigkeit zur Perspektivenwahrnehmung“ kodiert. Hierbei wurden sowohl Aussagen aufgenommen, die mittelbar als auch unmittelbar Einfluss auf diese Kompetenzentwicklung haben. Herauszustellen ist beispielsweise, dass die Jugendlichen teilweise explizit politische Aktionsformen wählen, um auf den Wert von Meinungspluralität hinzuweisen und um stereotype Denkmuster abzubauen.

Dann haben wir noch so im Allgemeinen über Vorurteile aufgeklärt, also, mit Fakten, also, wir haben das, glaube ich, genannt, Fakten gegen Vorurteile', und dann so Sachen wie die Kriminalitätsrate von Ausländern und Jobsuche und so weiter dargestellt. Oder auch zum Beispiel die Hartz-IV-Sache, wie viel die bekommen bzw. was das Klischee oder das Vorurteil ist, was die bekommen, und wie viel sie aber wirklich bekommen und das dann aber auch vergleichen mit dem Deutschen. (RONJA: 122-127)

Motivation für das eigene Engagement war es für RONJA und ihre Mitstreiter*innen in der schulischen Jugendinitiative, gegen Vorurteile anzugehen und 
ihren Mitschüler*innen neue Perspektiven aufzuzeigen. Sie ergänzt im Laufe des Interviews die oben zitierte Aussage wie folgt:

Es war so, dass wir gerne ein paar Projekte ansprechen wollten - also in der Zeit hat es uns total genervt, dass so viele Vorurteile kommen - wie: Die sind so kriminell und vergewaltigen unsere Frauen. Also, die üblichen, sodass wir die aufklären wollten und das Ganze hat sich irgendwie gut ergeben. (RONJA: 258-262)

Doch nicht nur die Aktionsformen sensibilisieren dafür, Vorurteile abzubauen und den Wert von Multiperspektivität anzuerkennen. ESRA, Schülerin der Sekundarstufe I, betont, dass es ihr vor allem durch die Arbeit in der Antirassismus-AG gelungen sei andere Perspektiven bewusster wahrzunehmen.

\begin{abstract}
Aber eher ist es positiv, dass ich sage: Okay, jetzt habe ich auch ein anderes Bild, ich kann es auch von einer anderen Perspektive angucken. Aber ich darf dabei nicht vergessen, und das, da versuche ich mich immer daran zu erinnern, ich darf dabei nicht vergessen, auch von anderen Perspektiven zu gucken. Also, ich gucke zwar von einer Perspektive, aber ich darf nicht vergessen, die anderen Perspektiven auch zu beachten und auch zu akzeptieren. (ESRA: 375-381)
\end{abstract}

Die Arbeit in der politischen Arbeitsgemeinschaft hat ESRA nach eigener Aussage dafür sensibilisiert die Perspektiven Andersdenkender wahrzunehmen. Auch LAURENZ verweist darauf, dass er erst durch sein politisches Engagement gelernt habe, offen für andere Perspektiven auf gesellschaftspolitische Themen zu sein:

Ich bin, meiner Meinung nach, insgesamt offener geworden, offener gegenüber Neuem. Ich habe früher ja auch politisch viele Sachen einfach anders gesehen. Ich bin viel offener gegenüber armen Menschen beispielsweise, und kann, glaube ich, deren Schicksale irgendwie jetzt besser irgendwie nachvollziehen und verstehen, irgendwie. Also, jetzt vorher zum Beispiel, wo ich das dann eher vielleicht eingleisig irgendwo gesehen habe und dass ich die ganze Sache irgendwo nicht so gut von allen Ecken und Enden verstehen konnte. (LAURENZ: 332-339)

LAURENZ ist überzeugt, dass er durch sein Engagement in der Jugendbewegung mehr Empathie und Verständnis für individuelle Schicksale entwickelt hat (LAURENZ: 341).

Doch wenngleich nahezu alle Befragten - zumeist ohne dass Multiperspektivität explizit thematisiert wurde - den Wert von Meinungsvielfalt betonen, so definieren sie auch klar die Grenzen der Bereitschaft zur Perspektivenübernahme. FRANZISKA erklärt beispielsweise, dass das Zulassen von politischen 
Aktionen und Diskussionen für sie auch bedeutet, die ganze Pluralität des Meinungsspektrums abzubilden. Und dennoch findet sie, dass es Grenzen geben muss. Rassismus und Sexismus sind für sie inakzeptabel.

\begin{abstract}
Ich hatte auch durchaus meine Diskussionen mit Leuten von den Jungen Liberalen oder der Jungen Union und mit denen diskutiere ich auch gerne. Ich habe auch oft erlebt, dass ich Leute von denen von irgendwas überzeugen konnte. Mir hat zum Beispiel mal jemand von der Jungen Union erzählen wollen, Atommüll sei überhaupt kein Problem, man könne das ja einfach nach Afrika verkaufen. Die Diskussion mit dem hat mir total viel Spaß gemacht, ehrlich gesagt, da sein Argument zu vermöbeln. Und das war auch gut und das hat auch andere weitergebracht, die das mitbekommen haben und eben da kann man ja auch eine Lösung finden. Ich finde, es gibt aber Grenzen, und ich finde das auch im Rahmen von einer Schule wichtig, dass man da zum Beispiel festlegt, dass politische Differenzen, dass Pluralität, dass Toleranz, dass verschiedene Meinungen total erwünscht sind. Aber dass man eben, wie wir es auch in unserer Jugendbewegung hier machen, auskommen will ohne Rassismus. Weil ich finde, dass Rassismus oder Sexismus, dass Diskriminierung keine Meinung, jedenfalls keine irgendwie legitime Meinung ist und da kann man auch sagen: Das akzeptieren wir nicht! (FRANZISKA: 1044-1059)
\end{abstract}

Auch BEN betont, dass rechte und rassistische Hetze für ihn keine legitime Meinungsäußerung darstellen: „Keine Ahnung so, rechte und fremdenfeindliche Hetze ist für mich keine Meinung, sondern tendenziell ein Verbrechen. " (BEN: 404-406)

Wenngleich FRANZISKA und BEN sich selbst eher als links-liberal eingestellt beschreiben, so halten sie es für wichtig, dass verschiedene Positionen auch in der Schule offen formuliert werden dürfen. Maßgeblich für die Grenzen dessen, was im Sinne der Meinungsfreiheit zu tolerieren sei, seien Grund- und Menschenrechte. Politische Diskurse an der Schule dürften keinen Raum für die Verbreitung diskriminierender Positionen schaffen. Auch HELGE, der in Sozialen Netzwerken wie Instagram politische Diskurse anregt, aber darüber hinaus auch an seiner Schule Anlässe für einen offenen politischen Meinungsaustausch schaffen möchte, betont den Wert der Schule als einen Ort, an dem junge Menschen, die sich nicht für Politik interessierten, auch mit politischen Themen konfrontiert würden. Sie lernten dadurch andere Meinungen und Sichtweisen kennen.

In der Schule, vor allem im Unterricht. Ich habe das Glück, dass ich viele Lehrer habe, die sich an den Lehrplan halten, aber trotzdem versuchen, diese Themen mit Politik in Verbindung zu bringen. Zum Beispiel, wenn wir in Deutsch Bücher lesen und Charakter analysieren, haben wir das im Zusammenhang mit der Flüchtlingskrise gemacht und ein Buch über einen Flüchtling gelesen. Ich denke, da kann man sich sehr gut mit den Leuten auseinandersetzen, die sich auch damit auseinandersetzen wollen. Vor allem in der Schule kriegt man natürlich auch, wenn man es im Unterricht 
macht, die Meinung anderer zu hören, die sich ansonsten eher nicht so sehr mit dem Thema auseinandersetzen. Weil sie eben dann in der Schule damit direkt konfrontiert werden. (HELGE: 238-248)

BEN betont darüber hinaus die Notwendigkeit, verschiedenen politischen Positionen in der Schule Raum zu geben. „Und man muss irgendwie Perspektiven vermitteln, die es gibt in der Politik. Und dann muss man, müssen die Schüler und Schülerinnen ein bisschen selber entscheiden, wie sie jetzt damit umgehen, ob sie das jetzt aktiv weiternehmen. " (BEN: 662-665)

Zusammenfassend lässt sich feststellen, dass sich die politisch aktiven Schüler*innen intensiv mit der Bedeutung von Perspektivenwahrnehmung und Multiperspektivität auseinandersetzen. Sie betonen, dass ihnen erst die Auseinandersetzung mit verschiedenen Positionen die Weiterentwicklung der eigenen Ideen und der eigenen politischen Sichtweisen ermögliche. Grundsätzlich bezeichnen sich die politischen Gruppen als offen, aber die Schilderungen von SOPHIE und KATJA lassen auch erkennen, dass es vor allem bei den außerschulischen Jugendgruppen eine Diskrepanz gibt zwischen dem postulierten Selbstverständnis politischer Offenheit und dem tatsächlichen Verhalten. So berichten Jugendliche, die in außerschulischen Gruppen aktiv sind, davon, dass zwar grundsätzlich eine Offenheit für andere Perspektiven proklamiert werde, aber die Bereitschaft, sich den Sichtweisen politisch Andersdenkender tatsächlich zu öffnen, teilweise gering sei.

Ein besonderes Potential scheinen daher politische Arbeitsgemeinschaften und Initiativen zu haben, die sich im Kontext der Schule organisieren. Strukturell bedingt ist die Zusammensetzung der Aktivist*innen hier in Bezug auf die politischen Positionen heterogener. Den Erfahrungsberichten der Schüler*innen ist $\mathrm{zu}$ entnehmen, dass dies anfänglich als anstrengender empfunden wird, aber insgesamt eine größere Offenheit für vielfältige Perspektiven ermöglicht.

Es lässt sich konstatieren, dass die Notwendigkeit politischer Vielfalt einhellig anerkannt wird. Auch politische Gegner*innen werden als relevante und ernst zu nehmende politische Akteur*innen wahrgenommen. Dabei unterscheiden die politisch aktiven Schüler*innen allerdings zwischen politisch legitimen und moralisch nachvollziehbaren Positionen. Unabhängig davon, in welchem Kontext (schulisch/außerschulisch) sie aktiv sind, betonen die Jugendlichen, dass sie durch ihr politisches Engagement sensibilisiert wurden für vielfältige Positionen.

Die interviewten Jugendlichen setzen für die Bereitschaft zur Perspektivenwahrnehmung und Perspektivenübernahme auch klare Grenze: Fremdenfeindliche Hetze, Sexismus und Homophobie sind für die Schüler*innen inakzeptabel. Einhellig wird betont, dass die Schule ein Raum sei, der die Möglichkeit biete, mit 
vielfältigen politischen Positionen in Kontakt zu kommen. Die Schüler*innen sind überzeugt, dass die Schule sich öffnen sollte für politische Positionierung, für das politische Handeln der Jugendlichen, aber dabei eben auch Grenzen setzen muss, die sich an den Grund- und Menschenrechten orientieren.

Neben analytischen Fähigkeiten und einer damit verbundenen kritisch reflexiven Auseinandersetzung mit Informationen ist für politische Bildungsprozesse von Bedeutung, welche Auswirkungen diese Fähigkeiten auf die Urteilsbildung der Individuen haben.

\subsubsection{Urteilsbildung und Kritik}

\subsubsection{Urteilsbildung zwischen „gesellschaftlichem Sein und Bewusstsein"}

In der Politikdidaktik wird die Notwendigkeit vielfältiger Fähigkeiten und dabei insbesondere die Bedeutung der Perspektiven-/Rollenübernahme, des sozialwissenschaftlichen Analysierens und der Konfliktfähigkeit für die Urteilsbildung betont. Diese ist eng verknüpft mit politischer Handlungsfähigkeit, da politisches Handeln ohne eine elaborierte Positionierung in Bezug auf politische Situationen kaum möglich ist (Sander 2008, 91). Im Folgenden werden daher die Aussagen der Schüler*innen analysiert und strukturiert, die auf eine (gelingende) Urteilsbildung schließen lassen.

Alle befragten Schüler*innen haben direkt oder indirekt beschrieben, dass ihr politisches Engagement ihre Urteilsbildung geprägt hat. Die Mittelstufenschülerin LEA bringt es auf den Punkt: „Zum einen ist es halt wirklich so, dass man sehr viel dadurch lernt, unterbewusst einfach, weil man zum Beispiel lernt, sich eine eigene Meinung zu bilden oder sich selbst zu reflektieren[.]" (LEA: 837-843) Dies ist nicht zuletzt darauf zurückzuführen, dass Analysekompetenzen gestärkt werden und Perspektivenvielfalt von den Akteur*innen als wertvoll empfunden wird. Vor allem die Auseinandersetzung mit Andersdenkenden bewegt die politisch aktiven jungen Menschen dazu, die eigene Position zu überdenken und zu reflektieren.

Es bereichert einen sehr, weil man andere Meinungen hört. Und wenn man andere Meinungen hört, die nicht mit den eigenen übereinstimmen, dann setzt man sich natürlich damit auseinander und überlegt, wie jemand dazu kommt. Woher holt er sich die Informationen, die ihn zu dieser Meinung führen? Und teilweise kommt man dann natürlich auch zu dem Punkt, wo man auch über seine eigene Meinung nachdenkt, weil man eben Gegenpunkte erlebt und hört und dann natürlich auf Kritikpunkte aufmerksam wird, die man vielleicht vorher noch nicht so gesehen hat. Und je mehr man sich natürlich dann damit auseinandersetzt, desto breiter wird auch die eigene Meinung und desto 
breiter gefächert natürlich auch das eigene Wissen. Und je breiter das Wissen ist, desto besser die Meinung, die man sich natürlich auch bilden kann. (HELGE: 261-271)

Die Tatsache, dass Meinungspluralität grundsätzlich als wertvoll und als notwendiger Bestandteil eines konstruktiven politischen Diskurses verstanden wird, führt dazu, dass sich die Jugendlichen auch kritisch mit der teilweise unzureichenden Meinungspluralität in der eigenen Gruppe auseinandersetzen. Für SOPHIE ist es wichtig, dass nicht immer nur vermeintliche Einigkeit besteht, sondern in der Gruppe immer auch Bedenken geäußert werden dürfen. Sie ist überzeugt, dass dies die Grundlage für eine gemeinsame Meinungsbildung sei.

Mal schauen, wir wollen am Montag noch ein bisschen diskutieren und noch einmal diese ganzen Forderungen besprechen, weil wir einen Bildungsstreik organisieren wollen und dann diese ganzen Forderungen noch einmal überarbeiten und überdenken. Keine Ahnung, ich hoffe, dass das schon vielleicht ein bisschen hitziger wird (lacht). Ich finde es nicht immer ganz gut, wenn man immer, Ja und Amen ' zu allem sagt, sondern dass wirklich jeder auch mal so seine Bedenken äußert und so etwas. (SOPHIE: 258-264)

Später im Verlauf des Interviews erklärt sie, warum ihr diese Offenheit so wichtig ist. Die Berufsschülerin betont die Notwendigkeit unterschiedliche Perspektiven anderer zu verstehen. Sie habe aber gleichzeitig das Gefühl, dass einige Aktivist*innen der Gruppe, in der sie sich engagiere, daran kein Interesse hätten. Wenngleich sie die politischen Ansichten ihrer Mitstreiter*innen teile, sei es für SOPHIE essenziell, die Motive anderer politischer Positionen im Blick zu behalten.

Manche haben dann schon gleich von vornherein so ihre Standpunkte, wo ich mir dann denke: Okay, jetzt höre es dir doch erstmal an und schaue es dir von allen möglichen Seiten an und sage nicht gleich schon wieder von Anfang an, was richtig oder falsch ist. Oder was, ja, manchmal habe ich so bei manchen Leuten echt das Gefühl, dass sie es vielleicht selber, wenn sie darüber nachdenken würden, bestimmte Sachen so gar nicht unbedingt als schlecht oder so etwas abstempeln würden, aber aus ihrer politischen Meinung heraus gleich sagen: okay, das ist schlecht, das kann man nicht machen. Und dann gar nicht bedenken, was da noch alles mit dranhängt, was es da noch für Parameter gibt, die man noch beachten muss und die da mit reinfließen. Und dass man deswegen manche Sachen so machen muss und ja vielleicht aufgeweichter und liberaler oder so etwas und nicht so radikal oder so. (SOPHIE: 316-332)

Diese reflektierte und (selbst)kritische Auseinandersetzung mit der Offenheit für andere politische Ansichten in der eigenen Gruppe zeigt, dass sich SOPHIE nicht 
von der vermeintlichen Einigkeit in der Gruppe vereinnahmen oder überwältigen lässt. Auch die deutlich jüngere ESRA reflektiert auf einer sehr abstrakten Ebene den Einfluss der Gruppe, in der sie sich engagiert. Auch sie hat keine grundsätzlichen Zweifel an den politischen Positionen der anderen aktiven Schüler*innen in ihrer selbstorganisierten Antirassismus-AG, aber sie beschreibt eine Gefahr, die sie in einer unbewussten Prägung einer Gruppenmeinung sieht. ESRA beschreibt sich als ,politisch links', möchte aber nicht, dass ihre Meinung durch das „Linksdenken der Gruppe“ geprägt wird.

Was ich aber sagen will, ist, dass durch die AG das nicht immer ganz positiv ist, weil die AG meine Gedanken manchmal sogar lenkt. Also, dass ich sage, dass ich diese Themen immer nur von der negativen Seite angucken muss, dass ich sage: Oh, ja. Jetzt gibt es wieder die Diskriminierung, und jetzt ist wieder, mmmh, aber vielleicht ist, mir fällt jetzt gerade kein Beispiel ein, aber vielleicht gibt es ja auch positive Sachen daran. Aber dadurch, dass wir so gelenkt werden, durch dieses, ja, Linksdenken, würde ich mal sagen, dass ich manchmal vielleicht sogar vergesse, was meine eigene Meinung dazu ist, weil ich einfach sozusagen mit diesen Werten dort aufgezogen und aufgewachsen bin. (ESRA: 366-375)

Auch SOPHIE reflektiert den Einfluss der Gruppe auf ihre individuelle Urteilsbildung. Sie merkt, dass die Gruppe ihr politisches Urteil prägt, auch ihr Denken beeinflusst. Sie ist froh über die Denkanstöße, will sich aber auch nicht beeinflussen lassen.

\begin{abstract}
Ich war auch ein bisschen schockiert darüber, also, dass das so mein Denken beeinflusst auf jeden Fall. Ich meine, es ist ein Schülerbündnis, aber es sind eben auch, es ist schon linksorientiert und es sind natürlich auch Leute dabei, die radikal links, also linke Positionen vertreten. Und ja, man fängt an, also, ich habe mir früher auch immer gedacht, so Linke, okay, das ist auch nicht so mein Fall, keine Ahnung. Manchmal hat man so gehört, radikal links oder radikal rechts ist irgendwie so dasselbe und deswegen, keine Ahnung, so radikal links war für mich auch so: Was ist denn das für eine? Keine Ahnung, man übernimmt dann schon irgendwie diese Position und man versteht die Leute auch besser und man kann sie auf jeden Fall besser nachvollziehen und man merkt auch: Scheiße, es ist nicht alles so wunderbar hier, auch mit unserer Demokratie und so etwas. Man darf trotzdem auch nicht immer, man muss trotzdem aufpassen, dass man sein eigenes Denken nicht auch vergisst. Ich bin schon froh über diese neuen Anstöße, und auch über diese kritischere Weltanschauung. Vor allem muss ich aber aufpassen, dass ich trotzdem auch nicht vergesse, selber zu denken, was schwer ist, wenn man wenig Schlaf bekommt (lacht). (SOPHIE: 411-426)
\end{abstract}

Insgesamt deuten die Aussagen aller interviewten Schüler*innen darauf hin, dass sie sich der Wirkung der Gruppe auf die Beurteilung politischer Fragen bewusst 
sind. Der Einfluss gruppendynamischer Prozesse auf ihre Urteilsbildung beschäftigt die Schüler*innen und sie erkennen die Gefahren, die damit verbunden sind. Wenngleich die Jugendlichen sensibilisiert sind für den Einfluss ihrer Mitstreiter*innen auf ihre individuelle Urteilsbildung, so betonen sie insgesamt aber den Mehrwert des politischen Engagements in der Gruppe und verschiedener politischer Aktionsformen.

Immer wieder wird das eigene politische Engagement der Jugendlichen mit dem individuellen Bedürfnis begründet, einen Beitrag für eine bessere Gesellschaft, eine bessere Welt zu leisten. KATJA erklärt ihre Motivation für ihre Mitwirkung in politischen Aktionen auch mit der moralischen Verpflichtung, alles dafür zu tun, die Welt ein bisschen besser zu machen.

Ja, das Oberziel ist dieses Utopische, alle können irgendwie gleichberechtigt nebeneinander leben, wo ich auch weiß, dass es wahrscheinlich nicht passieren wird. Aber weil ich denke, dass es das Beste wäre, was der Welt passieren könnte, könnte ich mich nicht nicht dafür engagieren, weil ich mich einfach total blöd fühlen würde und das Gefühl hätte, so: was mache ich hier eigentlich? Und manchmal ärgere ich mich auch darüber, dass das so ist, weil ich viel lieber zu McDonald's gehen würde und mir einen Burger reinpfeifen würde. Aber ich will irgendwie die Welt besser machen, weil ich denke, dass das meine Pflicht ist, weil ich hier lebe und darüber nachgedacht habe, dass das so wie es jetzt ist, nicht gut ist. Und es macht auch ein bisschen Spaß, aber das nicht immer. (KATJA: 433-443)

Ganz konkret werden diese moralische Ebene und ihr Einfluss auf das politische Handeln der Kinder und Jugendlichen am Beispiel einer Initiative von LEA. Sie veröffentlichte einen offenen Brief an ihre Mitschüler*innen und an die Lehrkräfte ihrer Schule. Ihr Ziel: Möglichst viele Familien sollten als Vormünder Verantwortung für unbegleitete minderjährige Geflüchtete übernehmen. Sie begründet diesen Apell vor allem mit der gesellschaftlichen Wirkung. So wären unbegleitete minderjährige Geflüchtete, die einen deutschen Vormund hätten, in der Mitte der Gesellschaft und nicht an den Rand gedrängt. Dies würde auch dazu führen, dass die Brücken zu Menschen mit Fluchterfahrung leichter zu schlagen sind.

Wenn jeder Geflüchtete einen Vormund hätte, wäre es, glaube ich, einfach ein komplett anderer Umgang mit Flüchtlingen, einfach generell in der Gesellschaft. Weil meistens ist es, auch wenn viele Leute sagen, sie finden es nicht so, bei vielen Leuten ist es einfach so, dass sich viele Leute gestört haben oder Angst haben vor den Flüchtlingen und sich irgendwie eingeschränkt fühlen durch die Flüchtlinge. Aber wenn man selbst Kontakt mit Flüchtlingen hat, glaube ich, werden einfach irgendwie Kontakte gesetzt und Brücken gebaut, die dann so die Kulturen verbinden und irgendwie Ängste nehmen und man sich dann nicht mehr eingeengt fühlt und man miteinander lebt. Wenn man miteinander lebt, fühlt man sich nicht eingeengt. (LEA: 881-890) 
Neben diesem Fallbeispiel aus dem Interview mit LEA ist allen Interviews gemein, dass die Schüler*innen von einem solidarischen Gesellschaftsverständnis geprägt sind. Bemerkenswert ist aber, dass sich dieses Selbstverständnis nicht beiläufig entwickelt, sondern teilweise sehr bewusst reflektiert wird. Besonders eindrücklich wird dies in den Ausführungen von FRANZISKA, die die Herausforderungen für eine begründete Werturteilsbildung wie folgt zusammenfasst:

Ich versuche das zu erklären, mir fällt das gerade schwer. Ich glaube, dass im Prinzip das gesellschaftliche Sein das gesellschaftliche Bewusstsein des Menschen bestimmt. Die Lebensumstände, die du hast, bestimmen auch deine Art und Weise, wie du denkst, weil du dein Verhalten ja auch irgendwie rechtfertigen musst vor dir selbst. Die meisten Menschen halten sich ja auch für moralisch. Also, ich glaube, ich kenne niemanden, der von sich behauptet: Ich bin ziemlich egoistisch und asozial. Man versucht, für jegliches Verhalten, von dem man tief in sich drin weiß, dass das eigentlich nicht in Ordnung ist, irgendwie eine Rechtfertigung zu finden. Dass man zum Beispiel sagt: ja, mir schenkt doch auch keiner was. Oder: das hätte der doch genauso gemacht. Oder: der verarscht mich doch auch ständig. Oder so: ach ja, keine Ahnung, habe ich das hier jetzt geklaut, aber das tut dem Aldi jetzt nicht weh. Deswegen glaube ich, dass das kein Prozess ist, den man voneinander trennen kann, sondern dass auf dem Weg zu einer besseren Gesellschaft die Menschen sich mit verändern. Das greift sozusagen ineinander über. Auch wenn der eine Prozess manchmal weiter fortgeschritten ist als der andere, holt sich das gegenseitig ein. Ich glaube, das sind zwei Sachen, die gleichzeitig passieren, die sich gegenseitig bedingen und nicht eins zuerst und dann das andere. (FRANZISKA: 400-417)

FRANZISKA beschreibt dabei ein Prinzip, bei dem das „,gesellschaftliche Sein “, die Lebensumstände, sozioökonomische Voraussetzungen und Privilegien, auch das Werturteil und damit das moralische Handeln der Menschen beeinflusst. Ohne direkt auf Urteilsbildung als zentrale Kompetenz der politischen Bildung angesprochen zu werden, offenbart diese Schilderung ein komplexes Verständnis der Entwicklung eines politischen Bewusstseins.

Der Blick auf das Selbstverständnis des gesellschaftlichen Zusammenlebens prägt vor allem die Werturteilsbildung der Kinder und Jugendlichen und ist nachhaltig vom politischen Engagement geprägt. HELGE beschreibt das wie folgt: „Ich möchte auf jeden Fall etwas machen, was mich selbst erfüllt und wo ich sage, ich lebe nicht nur für mich, sondern auch für andere Menschen. Vor allem auch die Zukunft [...]. Der Blick darauf ist sehr viel anders geworden. " (HELGE: 337-340) Dabei heben die befragten Schüler*innen vor allem die Tatsache hervor, dass sie durch das politische Engagement dazu veranlasst werden, sich mit realen politischen Problemlagen zu befassen. Es ist ihnen dabei wichtig, über 
die Ebene einer ausschließlich sachlichen Auseinandersetzung mit gesellschaftspolitischen Problemen hinauszugehen und politische Urteile sachlich, aber auch moralisch begründen zu können. Anders als im Unterricht empfinden sie den Meinungsbildungsprozess im Kontext der von ihnen gewählten Aktionsformen als authentisch und grenzen diese Erfahrungen daher deutlich von Erfahrungen im Politikunterricht $\mathrm{ab}$.

\title{
4.3.3.2 Wert realer und nicht simulierter Meinungsbildung
}

SOPHIE betont, dass sie sich durch das politische Engagement in der Jugendbewegung mit politischen Themen intensiver befasst und sich im Zuge dessen erstmals eine politische Meinung gebildet hat.

\begin{abstract}
Ich habe mir letztens gedacht, ich habe zum ersten Mal wirklich so etwas Ähnliches wie eine Meinung zu bestimmten Sachen, auf jeden Fall. Weil dadurch, dass ich es vorher einfach gemieden habe, habe ich auch wenig darüber geredet und mich wenig mit Politik oder mit politischen Ereignissen, gesellschaftlichen Ereignissen irgendwie beschäftigt. Und ja, ich glaube, ich hatte da auch nie wirklich eine Meinung dazu. Und das ist irgendwie schon, ich weiß auch nicht, ob ich jetzt so etwas wie eine, ich weiß nicht, unterscheidet man zwischen Meinung und Standpunkt? Also, ich würde sagen, vielleicht habe ich eine Meinung, aber noch keinen richtig 100\%igen Standpunkt. (SOPHIE: 375-388)
\end{abstract}

In der Schule ist es dem Politikunterricht nicht gelungen, das Interesse für politische Themen bei SOPHIE zu wecken. Dies kann auch darauf zurückzuführen sein, dass der Politikunterricht noch immer zumeist als Institutionenkunde wahrgenommen wird (hierzu ausführlich in Abschnitt 2.2.2.2 „Richtiges Politikwissen?“). Exemplarisch sei hierfür eine Aussage von FRANZISKA angeführt, die darstellt, wie in ihrem Politikunterricht das Thema Europäische Union behandelt wurde:

Und dann haben wir mithilfe einer Broschüre von der Konrad-Adenauer-Stiftung dann das Thema „Konrad Adenauer und die europäische Einigung “ durchgenommen und mussten dann die Jahreszahlen auswendig lernen. Also, ich hatte eigentlich keinen Politikunterricht, in dem es mal zur Debatte gekommen wäre oder in der irgendwie eine Meinungsbildung im Vordergrund gestanden hätte. Das heißt, meine politische Bildung habe ich immer nur außerhalb der Schule eigentlich gehabt. (FRANZISKA: 931-937)

Die Aussage von FRANZISKA verdeutlicht, dass sie Urteilsbildung, zumindest im Sinne einer authentischen Meinungsbildung, nicht als Bestandteil ihres Politikunterrichts wahrgenommen hat. Die Aussagen von BEN lassen darauf schließen, dass sich bei ihm der Eindruck verfestigt hat, dass sich vor allem die Lehrkräfte 
anderer Fächer (keine Politiklehrkräfte) über politische Schüler*innen freuen, weil diese sich ein Urteil bilden können und sich aktiv einbringen. Am Beispiel des Englischunterrichts verweist er darauf, dass sich vor allem die politisch aktiven Schüler*innen auch zu Themen wie Rassismus oder Kolonialisierung äußern wollen und sich im Unterricht positionieren. Für Politiklehrkräfte, so beschreibt BEN seine Erfahrungen, seien politisch interessierte Schüler*innen zuweilen eher lästig (BEN: 683-690). BEN beschreibt einen fundamentalen Unterschied zwischen Urteilsbildung und Diskursen im Kontext des politischen Engagements und dem Lernort Unterricht. In der Schule würden die Argumente vorgegeben und Urteilsbildung simulativ erprobt. Dieses Verfahren erweckt bei BEN den Eindruck, als würden die Lehrkräfte den Schüler*innen nicht zutrauen, eigenständig Argumente zu finden und abzuwägen.

Fundamentale Unterschiede liegen halt irgendwie prinzipiell auch darin, dass, wenn wir Diskussionen in der Schule haben, dass wir dann die Argumente ausgeteilt bekommen und die dann diskutieren sollen. Also, wir kriegen Argumente, die sollen wir dann benutzen, weil ich irgendwie das Gefühl habe, man geht davon aus, dass wir selber keine haben. Und das ist halt in der Gruppe anders. Das ergibt sich auch daraus, dass wir in der Gruppe alle freiwillig da sind und in der Schule alle da sitzen müssen, was halt die Debatte in der Gruppe emotionaler, aber auch in vielen Fällen deutlich interessanter und auch informativer macht, als zum Beispiel in der Schule, wo man ein Blatt mit irgendeiner Theorie bekommt, nicht sagen darf, ob die richtig oder falsch ist, sondern die einfach vertreten muss. (BEN: 601-611)

BEN führt später im Verlauf des Interviews an, dass er das Gefühl habe, dass Schüler*innen in ihrer individuellen Urteilsbildung bevormundet würden und Kritik an bestehenden Verhältnissen nicht erwünscht sei. Dieser Umstand schränke Kreativität und freiheitliches Denken ein. Seine Aussage steht hier stellvertretend für mehrere Erfahrungsberichte von Schüler*innen, die für die vorliegende Studie interviewt wurden.

Und auch allgemein - irgendwie auch in der Schule - ein bisschen mehr auch den Schülern vertrauen, glaube ich, wäre wichtig. Ich habe irgendwie immer das Gefühl, dass man in der Schule sehr stark bevormundet wird, auch in politischer Meinung, also, das gesagt wird: Das ist der Rahmen, in dem ihr euch bewegen dürft, und wenn $i h r$ da rausgeht, dann ist das falsch. Und das finde ich persönlich einen katastrophalen Ansatz. Das schränkt ganz massiv ein, auch Kreativität und freiheitliches Denken. Und ich finde, das könnte man gut ändern. (BEN: 669-676)

BEN ist überzeugt, dass ihm erst das politische Engagement, die Erfahrung mit politischen Aktionsformen und die Arbeit in der Jugendgruppe einen Freiraum 
geschaffen hätten, um sich die politische Wirklichkeit sinnhaft zu machen und reale Verfahren der Meinungsbildung zu erleben. BEN ist überzeugt, dass die Schule diese Erfahrung systematisch unmöglich mache. Auch HELGE bestätigt diesen Eindruck und begründet seine Haltung mit einem Fallbeispiel. Bei einem Workshop an seiner Schule, den er maßgeblich mitorganisiert hat, sollten alternative politische Systeme und Ideologien diskutiert werden. Thematisiert wurden unter anderem der Anarchismus und der Kommunismus. Für HELGE wurde schnell deutlich, dass die Lehrkraft einen offenen Diskurs dazu nicht zulassen würde. Dennoch hat er sich positioniert und seine Meinung geäußert.

Und ich glaube, hätte ich da, also, ich habe das teilweise gemacht, dass ich da offen gesagt habe, was ich dazu denke, aber hätte ich das krasser gemacht, dann wäre das auf sehr, sehr viel Kritik gestoßen. Was es auch ist im Unterricht. Ich habe den Lehrer auch im privaten Unterricht, abgesehen von dem Workshop. Und seitdem ist dieses Verhältnis sehr gespannt und wirkt sich vielleicht auch ein bisschen auf meine Noten aus. (HELGE: 125-138)

Auch BEN (703-707) ist überzeugt, dass kritische Schüler*innen in der Schule nicht erwünscht seien. Für HELGE beeinflusst diese Erfahrung auch sein Verhalten in der Schule. Er ist überzeugt, dass er seine politische Meinung in der Schule nicht öffentlich machen könne, weil er dann Probleme bekäme. Das habe auch Einfluss auf seine Urteilsbildung im Kontext des formalen Lernens in der Schule und im Unterricht.

Ich glaube, beziehungsweise ich spüre das persönlich auch, dass ich auch im schulischen Alltag öfter mit Politik konfrontiert werde, auch im Unterricht, und ich das Problem habe, dass das nicht immer so neutral ist, wie ich es mir vorstellen würde oder wie ich es gerne hätte. Das heißt, wenn ich meine Meinung öffentlich kundtun würde, auch in der Schule, dann würde ich damit teilweise ziemlich Probleme bekommen. Kriege ich auch teilweise, das nehme ich auch in Kauf. Aber was so das Ansehen angeht und wie sich dann damit auseinandergesetzt wird, was ich denke, ist teilweise sehr kritisch und bringt mir nicht viel. Außer dass ich damit vielleicht Schüler zum Nachdenken anrege. (HELGE: 112-120)

Nahezu alle interviewten Schüler*innen berichten von ähnlichen Erfahrungen. Es wird deutlich, dass sie das Ergebnis der individuellen Urteilsbildung zu politischen Fragen in der Schule und im Unterricht oftmals nicht verbalisieren können oder gar dürfen. Unabhängig von den Konflikten auf individueller Ebene zwischen Lehrkräften und Schüler*innen, von denen der überwiegende Teil der befragten Jugendlichen berichtet, lassen sich aus den subjektiven Erfahrungsberichten auch strukturelle Zwänge identifizieren, in denen die Schule verhaftet zu sein scheint. 
So berichtet KATJA davon, wie wenig anerkennend der Umgang mit politisch engagierten Schüler*innen zuweilen ist. In ihrer Gruppe hatten sie sich entschieden, einen Aktionstag ,Schule gegen rechts“ zu organisieren. Thematisiert werden sollten der erstarkende Rechtspopulismus, Rassismus und Nationalisierungstendenzen in der Gesellschaft. Der Titel und das Thema für den Aktionstag war das Ergebnis einer politischen Urteilsbildung. Die Schüler*innen haben das Potential und die Gefahr von Radikalisierungsprozessen am rechten Rand des politischen Spektrums erkannt sowie die damit verbundene Wirkung bis in die Mitte der Gesellschaft. Wie gefestigt ihr politisches Urteil ist, wurde bereits sehr früh in der Phase ihres politischen Engagements auf die Probe gestellt, denn bereits der Titel für den Aktionstag „Schule gegen rechts“ wurde, so beschreibt es Katja, von einigen Mitschüler*innen sowie durch die Schulleitung kritisiert und zunächst abgelehnt. Eine Umformulierung habe im Raum gestanden, denn Gefahr ginge doch von „rechts“ und von „,links“ aus (KATJA: 313-318). Der Aktionstag durfte letztendlich weiterhin „Schule gegen rechts“ heißen, die Arbeitsgemeinschaft haben die Schüler*innen dann aber umbenannt. Wenngleich diese Erfahrung zunächst mit Frustration (ausführlich zu den Frustrations- und Anerkennungserfahrungen in Abschnitt 4.3.5.1) verbunden war, so bot es auch einen wertvollen Lernanlass für politische Bildung, der aber nur in Ansätzen genutzt wurde. „Wir sind nicht tiefer auf die Begriffe eingegangen " (KATJA: 316) fügte KATJA im Interview noch an und offenbarte damit, wie eine große Chance die Erfahrungen der politischen Aktion als Bildungsgelegenheit aufzuarbeiten, auch bei autonomen Jugendgruppe, die sich an der Schule verorten, zuweilen ungenutzt bleiben.

Dennoch begleitet der Prozess der politischen Urteilsbildung die Erfahrungen in politischer Aktion in allen Phasen - ausgehend vom Interesse an politischen Themen, über die Planung und Umsetzung von politischen Aktionen bis hin zu dessen Reflexion. Oftmals ist der Akt der politischen Beteiligung auch Antrieb zur Analyse bestehender Verhältnisse und ihrer Beurteilung. Auch FRANZISKA beschreibt, wie ihr Interesse für politische Themen durch das politische Handeln gestiegen ist. Dadurch hat sie begonnen, sich mit Themen zu befassen, Informationen $\mathrm{zu}$ recherchieren und sich ein Urteil $\mathrm{zu}$ bilden. Sie führt das nicht zuletzt darauf zurück, dass das Politische nicht wie in der Schule herausgelöst als ein Thema unter vielen auf ihren Willensbildungsprozess einwirke, sondern als Selbstverständnis und zentraler Bestandteil.

Und mir hat das auch einfach total viel Spaß gemacht. Auch mein soziales Umfeld ist dann auch mein politisches Umfeld geworden. Am Anfang geht man vielleicht einmal in der Woche zu einem Treffen und alle paar Monate zu Demonstrationen. Aber irgendwann fühlt man sich auch verantwortlich, will auch eigene Ideen einbringen, bildet sich 
auch zu den Themen. Plötzlich ist da auch ein Interesse da. Ich habe über die Sachen dann nachgelesen, die wir da irgendwie bestreikt haben oder zu denen wir protestiert haben, und habe mir darüber auch eine politische Meinung bilden können. Das hätte ich in der Schule nie geschafft. Weil in der Schule Politik eben nur ein Randthema ist und man dann auch nichts inhaltlich bespricht. (FRANZISKA: 493-502)

Politische Urteilsbildung ist dabei nicht nur ein Prozess der Selbstbildung. Die Jugendlichen verfolgen auch das Ziel, Prozesse der Urteilsbildung bei anderen Jugendlichen zu initiieren. Beispielhaft dafür steht das Engagement von HELGE. Er ist aktiv in einer Gruppe, die einen politischen Instagram-Account betreut. Diesen Kanal möchte er nutzen, um seine politische Positionierung mit möglichst vielen Menschen zu teilen. Das Ziel der Gruppe ist es aber nicht, über den Kanal explizit politisch zu agitieren, sondern zu Diskussionen anzuregen. Wenngleich es sich um eine explizit antifaschistische Gruppe handelt, haben sie für ihre Arbeit Kontrollmechanismen eingeführt, die sicherstellen, dass die geposteten Beiträge einen offenen Meinungsaustausch ermöglichen. HELGE lernt dadurch, sich in dieser Gruppe nicht nur politisch zu positionieren, sondern in der Kommunikation politischer Themen eine Sprache zu wählen, die einen Diskurs ermöglicht.

\begin{abstract}
Damit setze ich mich dann auseinander und vor allem mit den anderen zusammen, eine Woche oder manchmal weniger, das kommt auf das Thema an. Dann schreibe ich dazu einen Text. Den diskutieren wir dann noch einmal, verändern den teilweise sehr stark, weil ich sehr gerne in diesen Texten stark meine eigene Meinung auch einbringe, weil das eben teilweise mehr zu Diskussionen anregt als wenn man einen sehr neutralen Text schreibt und ich eben auch meine eigene Meinung kundtun möchte. Das sehen aber viele nicht so. Deswegen verändern wir ihn eben teilweise sehr stark. Dann veröffentlichen wir den, das heißt, viele Leute können ihn lesen, teilweise sehr viele. Wir haben auch schon Texte gehabt, wo 200 Leute darunter diskutiert und kommentiert haben. (HELGE: 437-448)
\end{abstract}

Insgesamt kann konstatiert werden, dass das politische Handeln für die Jugendlichen Anlässe schafft, sich intensiv mit politischen Themen zu befassen. Das regt sie an, eine politische Identität und eine politische Positionierung zu entwickeln (HELGE: 381-390). Diesen Findungsprozess der eigenen politischen Identität durch die politische Aktion beschreiben alle interviewten Personen. Die damit verbunden Lernerfahrungen fasst KATJA mit der Entwicklung eines kritischen Bewusstseins zusammen, welches sich nicht durch den Politikunterricht oder die Schule entwickelt habe, sondern maßgeblich von ihren politischen Partizipationserfahrungen geprägt sei. 
Und (..) ich bin allgemein bei allen möglichen Sachen kritischer, also nicht nur was Politik angeht, sondern allgemein. Was ich auch eigentlich ziemlich gut finde. Und das wäre ich, glaube ich, nicht, wenn ich nicht viel in Richtung Politik und so gemacht hätte. Und das ist ganz gut (schmunzelt). (KATJA: 787-791)

FRANZISKA beschreibt, dass politische Aktionen und die Selbstorganisation in der Jugendbewegung für sie auch bedeuten, sich ein Urteil zu gesellschaftspolitischen Streitfragen zu bilden. Sie hebt dabei vor allem hervor, dass diese komplexe Selbstbildungserfahrung intrinsisch motiviert sei und nicht von Leistungsdruck oder Erfolgserwartungen torpediert werde, die sie mit schulischem Lernen verknüpft. Der Bildungsraum Jugendbewegung zeichne sich dadurch aus, dass es nicht ,um irgendeine Note oder um irgendein Zertifikat oder etwas, das man am Ende dafür bekommt " (FRANZISKA: 510) geht.

Vor allem diese authentischen Momente politischer Urteilsbildung kann der Politikunterricht allein nicht ermöglichen, aber als wertvolle Erfahrung und weiteren Lernanlass aufgreifen. JAN verweist darauf, dass der Politikunterricht vor allem dann einen großen Mehrwert habe, wenn man politisch interessiert und engagiert sei, weil er dabei helfen könne „Dinge rationaler zu sehen“ (JAN: 670) und ein pluralistischeres Meinungsbild zu entwickeln. Wenngleich der Politikunterricht rückblickend für die meisten interviewten Schüler*innen eher als langweilig und zu sehr orientiert an der Vermittlung von institutionenkundlichem Wissen verortet wird, betonen die Befragten, dass sie ihn vor allem seit ihrem selbstbestimmten politischen Engagement als einen wertvollen Bestandteil des ganzheitlichen politischen Bildungsprozesses wahrnehmen.

Es ist irgendwie so, dass, wenn man schon seine politische Meinung hat, dann ist der Unterricht noch einmal sehr gut, um bestimmte Dinge rationaler zu sehen und auch andere Meinungen sich dann auch einzuholen. Das sind so die Sachen, die ich hauptsächlich in diesem Unterricht sehe. Und natürlich, dass es Spaß macht und so, was dann natürlich damit zusammenhängt, dass es mich ziemlich interessiert. (JAN: 669-674)

BEN verweist darauf, dass ihm das politische Engagement dabei geholfen habe, die Theorien, die er in der Schule gelernt habe, einordnen zu können. Er versteht jetzt, warum die Theorien wichtig sind, betont aber, dass das vor allem dann passiere, wenn man ihren Wert erkenne. Er führt das auch auf die Erfahrungen in der politischen Aktion zurück, die er als bedeutsam für seine individuelle Fähigkeit zur politischen Urteilsbildung beschreibt. Er erkennt aber auch die Notwendigkeit, über den Politikunterricht mit neuen Perspektiven und theoretischen Zugängen konfrontiert zu werden. 
Also, mir hat Schule zu dem Zeitpunkt außerhalb davon etwas gebracht, als ich verstanden habe, wie das funktioniert. So ein bisschen im größeren Maßstab, so ein bisschen $z u$ denken. Also, man kann Theorien besser verstehen, man weiß, worauf die hinauswollen, und man weiß, was sie am Ende bezwecken möchten. Ich habe aber das Gefühl, das hilft am ehesten, wenn man eine gewisse Grundmeinung schon hat, und dann ein bisschen darauf eingehen kann, was man liest und was man lernt zum Beispiel. Also irgendwie, wenn ich jetzt was über Neoliberalismus lese, dann kann ich jetzt argumentieren, warum ich persönlich das kacke finde. Genauso könnte wer anderes, wenn wir jetzt beispielsweise Marxismus in der Schule machen, der sich jetzt als Neoliberaler versteht, vielleicht auch aus seiner Perspektive sagen, warum er Marx kacke findet. Und solche Sachen finde ich deutlich besser, also wenn man sich kritisch über Theorien auch äußern dürfte in der Schule. (BEN: 691-703)

Um die politische Urteilsbildung junger Menschen, die sich politisch engagieren, zu unterstützen und diesen Lernprozess politikdidaktisch zu begleiten, wäre eine enge Verknüpfung realpolitischer Erfahrungen mit theoretischen Konzeptionen und erlernten Strategien sinnvoll, betont auch RONJA. Ihr sei es wichtig, dass es ermöglicht werde, die im Unterricht entwickelten Kompetenzen in der Praxis zu erproben und die dabei gewonnenen Lernerfahrungen wieder im Unterricht zu reflektieren. Für RONJA sind reale politische Diskussionen angewandte politische Bildung. ,[A]lso, das, was man in der Schule lernt, Argument, These, Begründung und Beispiel, das kann man super anwenden. Und das ist cool oder das wäre cool, wenn es direkt verbunden wäre. " (RONJA: 1178-1183)

Der Unterricht in gesellschaftswissenschaftlichen Fächern kann dann für politisch aktive Kinder und Jugendliche auch als Wert erkannt werden, wenn sie hier einen Verwirklichungsraum für politische Diskurse erhalten. Wenn das gelingt, empfinden sie den Unterricht auch als wertvolle Ergänzung zu ihrem politischen Engagement. Exemplarisch sei hier auf die Beschreibungen von LAURENZ verwiesen. Er erklärt, dass er gerne Diskussionen führe und sich freue, wenn jemand eine andere Position vertrete, weil er dann seine Argumentations- / Überzeugungsfähigkeit trainieren könne.

Ich trete ziemlich oft in eine Diskussion mit dem Lehrer ein, was mir einerseits auch Spaß macht. Mir macht es ja auch Spaß, wenn jemand anderes seine Meinung konträr zu meiner hat und ich dann irgendwie ausprobieren kann, wie gut ich jetzt diskutieren kann, wie gut meine Argumente sind. Also, ich trete, wie ich gesagt habe, ich trete gerne in Diskussionen sowohl mit meinem Erdkunde-, als auch mit meinem Geschichtslehrer ein und gucke halt, wie weit ich auch die Sachen reizen kann, so weil mir das auch selbst Spaß macht und ich ja letztendlich, meiner Wahrnehmung nach, an jeder Diskussion irgendwo wachse, fast egal mit wem, also fast. (LAURENZ: 873-881) 
Oftmals beschreiben die Interviewpartner*innen aber, dass ihnen genau dieser Ermöglichungsraum nicht zugestanden wird. Die schulische politische Bildung verpasst dann die Möglichkeit, als Vermittlerin zwischen politischen Selbstbildungserfahrungen und politikdidaktisch begleiteten Lernorten $\mathrm{zu}$ fungieren. Dieser Brückenschlag scheint vor allem dann nicht zu gelingen, wenn politisch aktive Schüler*innen nicht nur ihr neu erworbenes Wissen in den Unterricht oder Schulalltag einbringen, sondern ihr politisches Urteil auch zu Kritik an bestehenden Verhältnissen führt. Spätestens diese verbalisierte Kritik führt, den Erfahrungsberichten der interviewten Schüler*innen folgend, zu Widerstand und Ablehnung durch die Lehrkräfte. Das folgende Unterkapitel stellt daher die Rekonstruktion der Bildungserfahrungen im Kontext von Kritik und Selbstreflexion in den Mittelpunkt.

\subsubsection{Kritik und (Selbst)Reflexion}

Die Berufsschülerin FRANZISKA formuliert deutlich ihre Kritik an gesellschaftlichen Verhältnissen. Sie und ihre Mitstreiter*innen wollen eine Welt, ,die gerecht ist, die frei ist von Ausbeutung, die frei ist von Zerstörung, Krieg, von Gewalt" (FRANZISKA: 727-728). Diese Grundüberzeugung hält die Gruppe zusammen. Für FRANZISKA ist darüber hinaus wichtig, dass mit der Kritik an den bestehenden Verhältnissen mehr verbunden sein muss, als nur die Verantwortung des Einzelnen hervorzuheben. Sie ist überzeugt, dass nachhaltige Veränderungen nicht nur dadurch erreicht werden, dass an den einzelnen Menschen appelliert wird. Mechanismen der Ausbeutung von Mensch und Natur lassen sich ihrer Ansicht nach nicht nur durch individuelles (Konsum)Verhalten überwinden. FRANZISKA verdeutlicht ihre Position am Beispiel der Umweltpolitik:

[W]enn mir das nicht reicht, dann wird mir immer gesagt, du kannst ja auch Konsumentscheidungen treffen. Du kannst dir ein Elektroauto kaufen, wenn dich das jetzt so aufregt mit dem Dieselskandal oder so. Meiner Meinung nach ist das aber nicht so. Also, ich persönlich kann mir sowieso kein Elektroauto kaufen, weil ich dafür gar kein Geld habe, sondern höchstens ein gebrauchtes Dieselauto. Das heißt die Wahlmöglichkeit steht mir gar nicht offen. (FRANZISKA: 262-268)

Die Berufsschülerin betont, dass grundsätzlichere Fragen von Machtverteilung diskutiert werden müssten (FRANZISKA: 268-279). Auch für den Gesamtschüler JOHANNES bedeutet Gesellschaftskritik vor allem die Überwindung von 
Ausbeutung und (ökonomischer) Abhängigkeit, die in seinen Augen ein Wesensmerkmal gegenwärtiger gesellschaftspolitischer Verhältnisse ist. Die Gesellschaftsutopie des Schülers ist gekennzeichnet von einer grundsätzlichen Kritik an Macht- und Herrschaftsstrukturen.

Ich denke, dass Selbstentfaltung und Selbstentwicklung ein ganz wichtiger Punkt dabei ist. Es ist wichtig, dass man dem Menschen das nicht vorschreibt, durch Leistungsdruck oder anderes, das und das musst du machen, da und da musst du hinkommen. Jeder Mensch hat irgendeine Form von Talent, es ist nur ganz schwer, die auszuschöpfen, weil oftmals sie von der Gesellschaft nicht gefördert werden. Wenn es jetzt aber eben keine feste Gesellschaft mehr gibt, keine wirkliche Struktur, keine Machtstrukturen, sondern jeder für sich leben kann, sich aber theoretisch zusammenschließen kann mit Leuten, dann steht es ihm frei, seine Talente zu entwickeln, er muss nichts mehr, also, er wird nicht mehr gezwungen etwas zu erreichen, er kann jetzt nur noch etwas erreichen. (JOHANNES: 139-148)

Im weiteren Verlauf des Interviews konkretisiert JOHANNES diesen kapitalismuskritischen Ansatz. Im gegenwärtigen politischen, vor allem aber ökonomischen System sei Demokratie immer verbunden mit einer Bevorteilung von wirtschaftlicher Freiheit vor individueller Freiheit. Deshalb muss man, davon ist JOHANNES überzeugt, immer zunächst erfolgreich im Wirtschaftssystems funktionieren, um auf Grundlage des ökonomischen Erfolgs individuell frei sein zu können. Dies befördere Egoismus (JOHANNES: 179-191).

Der überwiegende Teil der Schüler*innen kam in den Interviews direkt oder indirekt auf Macht- und Herrschaftsverhältnisse zu sprechen. Auch wenn dabei die Systemkritik nicht immer derart umfassend formuliert wird wie bei JOHANNES oder FRANNZISKA. Der Mittelstufen-Schüler HELGE übt vor allem Kritik an einem Demokratieverständnis, das an den eigenen Landesgrenzen endet. Werte wie Gleichheit und Freiheit zu proklamieren, aber dann mit Ländern politisch und wirtschaftlich zu kooperieren, die diese Rechte missachten, ist für ihn nicht nachvollziehbar.

Ich glaube, dass es in vielen Punkten nicht so umgesetzt wird, wie es vielleicht gedacht ist (..), und vor allem auf Länder bezogen, die nicht zu unserem Staat passen. Wenn man sagt, wir leben in einem demokratischen Land, dann gilt das ja vor allem für unser Land, aber nicht unbedingt für Menschen, die nicht in unserem Land leben. Und ich glaube, das ist ein großes Problem, weil wenn man sagt, wir leben in einem demokratischen Land, dann bezieht man ja andere Menschen nicht mit ein, die aber trotzdem mit diesem System sehr viel zu tun haben, zum Beispiel Leute, die für Firmen arbeiten, die in unserem Land entstanden sind oder sehr viel Steuern oder Ähnliches zu unserem Land beitragen. (HELGE: 81-90) 
Auch dieser Aussage ist eine globalisierungs- und kapitalismuskritische Perspektive zu entnehmen. Die Unzufriedenheit mit bestehenden Macht- und Herrschaftsverhältnissen kann auch Antrieb für die eigene politische Arbeit und damit auch für politische (Selbst)Bildungserfahrungen sein. FRANZISKA erklärt beispielsweise, dass ihr Bedürfnis nach gesellschaftlichen Veränderungen sie zu ihrem politischen Engagement motiviert. Ausschlaggebend für ihr Mitwirken in der politischen Jugendgruppe war ursprünglich die Sorge vor individueller Benachteiligung durch politische Entscheidungen. Als Schülerin der Mittelstufe setzte sie sich gegen die Verkürzung des Abiturs auf acht Jahre (sogenanntes G8-Abitur) und gegen Studiengebühren ein. Erst durch die Erfahrungen, die sie in den Bildungsstreikjahren 2009 und 2010 gesammelt hat, wurden ihr Dimensionen der Gesellschaftskritik bewusster. Sie sind seither ihr Antrieb, sich weiterhin politisch einzubringen, auch wenn die bildungspolitischen Ziele teilweise längst erreicht wurden.

Der große Bereich wäre zum Beispiel, wenn man wegzoomt von einer kleinen Sache, wo sich viele engagieren, zum Beispiel in der Schule für mehr Gleichheit oder gegen Nazis oder so, dass man sich insgesamt eine solidarische Gesellschaft wünscht, die nach Grundsätzen organisiert sind, wo es eben nicht darum geht, dass Leute zum Beispiel irgendwie, dass ihr Eigentum gesichert wird, dass sie besonders, irgendwie ihre Reichtümer aufhäufen können oder so. Sondern, dass man den Grundsatz der Solidarität nach oben hebt, dass es eben jedem gut gehen könnte, wenn man etwas grundsätzlich umorganisieren würde. Das ist etwas, dass ich irgendwie im Laufe der Jahre gewonnen habe, so einen Weitblick, weswegen ich auch weiter gemacht habe, nachdem ich dann vielleicht irgendwie die Sache erreicht hatte, dass bei uns hier die Studiengebühren nicht mehr da waren oder das G9-Abitur wieder eingeführt wurde. (FRANZISKA: 34-45)

Neben den hier bereits beispielhaft angeführten Ausführungen, die auf komplexe gesellschaftskritische Denkmuster hindeuten, bewegt ein Thema etwa zwei Drittel aller befragten Schüler*innen: Vertrauen in die Staatsgewalt. Insgesamt gibt es 24 Fundstellen in acht Interviews zu diesem Themenkomplex, obwohl kein einziges Mal explizit nach den Erfahrungen mit der Polizei gefragt wurde. Besonders eindrucksvoll ist hier die Antwort von JOHANNES zur Eingangsfrage, was ihm zu dem Begriff „Demokratie“ als erstes in den Sinn käme: „Polizeistaat“ (JOHANNES: 164). Es ist die erste Assoziation, die er mit dem Konzept Demokratie in Verbindung bringt. Im weiteren Verlauf führt er das genauer aus:

Keine Ahnung. Es wird jedes Mal gesagt, dass Polizei eines der wichtigsten Mittel ist, um die öffentliche Ordnung aufrechtzuerhalten. Ich habe aber auch schon so oft erlebt, wie Polizei einfach in Demos gerannt ist, Leute wahllos zusammengeschlagen hat, 
einfach so Leute verhaftet hat, wie der Staat Leute abschiebt, eben mithilfe der Polizei und wie, wenn irgendwer irgendwas gegen die Polizei sagt, vor Gericht nie irgendwas passiert ist. Und das ist für mich einfach eine grundsätzliche Ungerechtigkeit, die ich eben mit Demokratie auch verbinde und auch stark mit Demokratie verbinde. (JOHANNES: 172-179)

Ähnlich wie JOHANNES berichtet auch JAN von unverhältnismäßigen Einsätzen der Polizei auf Demonstrationen. Er bezeichnet Polizeigewalt als einen entscheidenden Radikalisierungsfaktor für seinen Politisierungsprozess: „Ich würde auch sagen, einer meiner Hauptradikalisierungsfaktoren ist die Polizei gewesen auf Demonstrationen. Das ist auf jeden Fall ein ziemlich wichtiger Faktor. " (JAN: 459461) In den Anfängen seines politischen Engagements hat er sich von konspirativ agierenden Gruppen wie Antifa-Gruppierungen eher distanziert. „Und jetzt bin ich da halt selber voll drin und laufe nun auch schwarz und schwarzgekleidet und vermummt auf Demonstrationen rum. "(JAN: 463-466) Es ist eine Art und Weise, auf Polizeigewalt und als Willkür empfundene Staatsgewalt zu reagieren.

Noch verbreiteter ist die Skepsis gegenüber interessierten Menschen, die der Gruppe nicht bekannt sind. Wie in Abschnitt 3.2 „Forschungssample“ beschrieben, war der Zugang zum Feld schwierig. Er war vor allem von Vertrauensarbeit geprägt. Mehrmals berichteten mir Schüler*innen, dass sie im Laufe ihres politischen Engagements sogenannte Anquatschversuche erlebt hätten. Nur eine Schülerin war auch bereit, davon im Interview zu berichten. FRANZISKA sagte, dass Mitgliedern der Gruppe unter anderem Geld angeboten wurde, damit sie von den Aktionen der Gruppe berichten (FRANZISKA: 873-880). Alle interviewten Jugendlichen vertreten ihre Positionen offen. Ihre Plenumssitzungen und AG-Treffen sind öffentlich, ihre Positionen veröffentlichen sie auch teils mit Namensnennung im Internet. Sie gehören nicht zu den Gruppen, die subversiv und staatsgefährdend sind. Sie stehen auch nicht in den Verfassungsschutzberichten der Länder. Dennoch: Aussagen und Erfahrungsberichte wie die von JOHANNES, JAN und FRANZISKA finden sich in vielen Interviews und wurden noch häufiger in den Vorgesprächen zu Interviews erwähnt, die letztlich - auch aufgrund des mangelnden Vertrauens in staatliche Institutionen - nicht zustande kamen. Der Politisierungsprozess der Jugendlichen ist nicht selten unmittelbar verknüpft mit dem Erleben von Polizei- beziehungsweise Staatsgewalt, die von den Schüler*innen als unverhältnismäßig eingeschätzt wird.

Es sind vor allem die Erfahrungen mit struktureller Gewalt, die den interviewten Schüler*innen auch dazu dienen Protestformen des zivilen Ungehorsams zu legitimieren. 
Jetzt oberflächlich betrachtet, gibt es natürlich einen Widerspruch. Und diese oberflächliche Betrachtung führt bei vielen Menschen ja auch dazu, dass sie im Grunde die antifaschistischen Aktionsformen als schlecht bezeichnen und letztendlich auch oft sagen: Die Antifa - das sind die wahren Faschisten. Und ich denke, dass diese oberflächliche Betrachtung nicht ausreicht und dass wir gucken müssen, in was für einer Gesellschaft wir leben. Und ich denke, dass wir in unserer Gesellschaft jeden Tag auf die eine oder andere Weise mit Gewalt konfrontiert sind. Ob es jetzt tatsächliche Gewalt von Nazis an Ausländern ist oder ob es eben strukturelle Gewalt ist, die auf uns ausgeübt wird. Über den Staat, über die Wirtschaft, über Unternehmen etc., ökonomische Gewalt, strukturell. Und ich denke, dass sich da die Gewaltfrage letztendlich gar nicht stellt, weil (..) ich denke, dass wir an einer gewissen Stelle auch Gewalt einsetzen können und müssen, um eben, es steht natürlich da, oberflächlich betrachtet eben ein Widerspruch, aber um eine friedfertige Gesellschaft zu erreichen, ja, aber diese Gesellschaft kann man eben nicht mit Nationalsozialisten, die offen ihren Hass zur Schau stellen, auf den Straßen marschieren, die kann man so nicht erreichen. Und ich denke, dass es da eben auch gerechtfertigt ist, diese auch zu blockieren und für die Polizisten stellt sich die Gewaltfrage ja auch nicht, beispielsweise. Wenn man dann einen Gegner hat, der friedfertig vorgeht, dann wird man gegen den Gegner auch friedfertig vorgehen. Wenn man aber einen Gegner hat, der von vornherein von sich sagt: nein, wir gehen gewalttätig vor, dann muss man ja auch in irgendeiner Weise etwas erwidern können. Also, das bedeutet halt auch, dass man Unterscheidungen zwischen Formen von Gewalt machen muss. Das bedeutet nicht, dass man wie die RAF herumläuft und wahllos reiche Menschen erschießt, oder Supermarktketten in die Luft jagt. Das ist überhaupt nicht das, was ich jetzt darunter verstehe. (LAURENZ: 631-656)

Diese ausführliche Darstellung und Rechtfertigung des Einsatzes von Gewalt, dabei explizit von passiver Gewalt in Form von Blockaden, zeigt, dass der Einsatz von Partizipationsformen des zivilen Ungehorsams nicht unreflektiert bleibt und es sich dennoch lohnt diese Argumentation als wertvollen Lernanlass für die formale Bildung aufzugreifen (ausführlicher in 4.3.4.5 ,Reflexion des eigenen Handelns“"). Schulstreiks, Demonstrationsblockaden oder die Verhinderung von Abschiebungen werden nicht als bloße Mittel zum Zweck verstanden. Sie werden argumentativ in einen ganzheitlichen Kontext von Machtverteilung und Machtmissbrauch eingebettet. Die durchaus komplexe Beurteilung bestehender Verhältnisse bietet die Chance, als Lernanlass für den Politikunterricht oder projektorientiertes Arbeiten zu dienen, wobei das Ziel nicht sein sollte, gegen die vermeintlichen Fehlkonzepte der Schüler*innen anzuarbeiten, sondern an ihren Erfahrungen anzuknüpfen. Macht- und Herrschaftsverhältnisse in der Gesellschaft können auch in der Schule kritisch hinterfragt werden. Didaktische Lernsettings können dazu dienen, die Argumentation weiterzuentwickeln und Perspektiven aufzuzeigen, die aufgrund der unmittelbaren Betroffenheit der Schüler*innen aus dem Blick geraten. Insgesamt zeigt sich, dass die Reflexion von Macht- 
und Herrschaftsverhältnissen auch in Bezug auf die individuelle und kollektive Urteilsbildung für die Schüler*innen eine große Bedeutung hat.

Darüber hinaus scheint Selbstreflexion den Prozess der politischen Positionierung fortwährend zu begleiten. LEA erklärt explizit, dass die Reflexion des eigenen Urteils für sie sehr wichtig sei. Für sie ist darüber hinaus aber auch bedeutsam, ihre eigenen Positionen mit denen ihrer Mitstreiter*innen abzugleichen. Auch um herauszufinden, ob sie tatsächlich selbst eine bestimmte Position vertritt oder es sich eher um das Ergebnis einer kollektiven Urteilsbildung handelt, hat sie gelernt, den Einfluss der Gruppe auf ihren Politisierungsprozess immer wieder neu zu hinterfragen.

Ja, ich glaube schon, weil es einfach irgendwie in einer AG oder wenn man sich generell mit Politik auseinandersetzt, immer wichtig ist, dass man selbst noch mal über seine eigene Meinung nachdenkt, und nicht nur über die eigene, sondern auch über die Meinung von anderen Leuten nachdenkt. Weil dadurch wägt man selbst noch mal ab, ob das, was man gesagt hat, nicht vielleicht doch irgendwie das ist, was man eigentlich findet, und ob es vielleicht doch eigentlich eine andere Meinung ist, die man vertritt. (LEA: 631-637)

Auch ESRA ist es wichtig, dass in politischen Diskursen immer offen argumentiert werden kann und niemand Sorge vor Ausschluss haben muss, nur weil eine andere politische Position vertreten wird. So hat sie es in ihrer Familie gelernt und erwartet diese Grundhaltung auch von ihrer politischen Arbeit in der Arbeitsgemeinschaft. Sie hat aber durchaus die Sorge, dass die Gruppe versuchen könnte Einfluss auf ihre Urteilsbildung zu nehmen.

Und meine Schwester und meine Mutter denken eher aufkommunistische Art und Weise und sie sind sehr, sie sind eigentlich schon Anhänger des Kommunismus. Und dadurch wird das auch ein bisschen geprägt, dass ich sage: Okay, ich denke vielleicht auch ein bisschen so. Aber bei mir zu Hause ist es trotzdem so, dass sie überhaupt nicht gegen mich sind, wenn ich eine andere Meinung habe. Ich weiß es nicht, ich habe es noch nicht ausprobiert, wie es zum Beispiel wäre, wenn ich mich in der AG so äußere, dass ich sage: Aber vielleicht habe ich doch eine andere Meinung. Ich glaube nicht, dass sie sauer sind, aber vielleicht versuchen sie mich zu lenken und sagen: Aber nee, deine Meinung ist eigentlich falsch. (ESRA: 450-459)

Die befragten Schüler*innen reflektieren aber nicht nur den Einfluss der Gruppe auf ihre individuelle Urteilsfindung, sondern beschreiben auch die Gefahr einer möglichen Indoktrination durch Lehrkräfte, die ihre selbstverwalteten Initiativen und Arbeitsgemeinschaften begleiten. Besonders hervorzuheben ist an dieser Stelle ein Erfahrungsbericht aus dem Interview mit ESRA. Sie beschreibt, wie 
eine Lehrerin, die sie sowohl aus der AG-Arbeit als auch aus dem Unterricht kennt, Einfluss auf die Meinungsbildung der Schüler*innen nimmt. Als Beispiel führt sie das Themenfeld Industrialisierung an. Interessant ist das Beispiel vor allem deswegen, weil die Lehrerin linke Positionen vertritt und sich ESRA selbst als politisch links verortet. ESRA findet die Lehrerin sympathisch und schätzt ihre grundsätzlich linke Haltung. Sie findet aber auch, dass es einen Unterschied zwischen ihrer politischen Haltung, ihrer Argumentation in der freiwilligen AG, die sie als Pädagogin begleitet, und dem Unterricht geben muss. (ESRA: 421-429)

Und es gefällt mir auch nicht, weil ich zum Beispiel (...4...) bei der Erfindung des Fließbandes sehe ich nicht nur positive Sachen oder so. Ich sehe auch sehr viel Negatives, dass man sich von seinem eigenen Produkt entfremdet, dass man nicht mehr das tun kann, was man selber will, sondern man hat jeden Tag dieselbe Bewegung. Aber, sobald diese Lehrerin dagegen ist und sagt, dass das aber eigentlich was Positives ist, versucht sie das auch den Schülern so beizubringen. Ich finde aber, dass die Schüler ihre eigene Meinung bilden sollten und dass jeder, dass man die Grundfakten sagt, egal aus welcher Perspektive, und die Schüler dann sagen können, ich bin dafür oder ich bin dagegen. Und das mag ich nicht an dem Unterricht. (ESRA: 429-439)

In jedem Fall zeigen die in diesem Unterkapitel aufgeführten Erfahrungsberichte, dass die Jugendlichen ihre politische Urteilsbildung und auch die Aktionsformen reflektieren. Eine ausführliche Analyse der Aktionsformen sowie deren Reflexion durch die Schüler*innen folgt in Abschnitt 4.3.4 „Partizipationserfahrungen und Konfliktfähigkeit“.

Urteilsbildung und Handlungsfähigkeit, darin ist sich auch die Politikdidaktik weitgehend einig, haben eine Vielzahl von Überschneidungspunkten, allerdings weist Wolfgang Sander (2008, 91) darauf hin, dass „politisches Urteilen auch ohne politisches Handeln möglich ist und andererseits das praktische politische Handeln auch Fähigkeiten verlangt, die sich nicht unmittelbar aus dem politischen Urteil ergeben“. Inwiefern die Erfahrungen in der politischen Aktion und der politischen Selbstorganisation Lernprozesse anregen, wird im folgenden Kapitel genauer untersucht.

\subsubsection{Partizipationserfahrungen und Konfliktfähigkeit}

\subsubsection{1 Öffentlichkeit herstellen und Organisationsfähigkeit}

Die in Abschnitt 2.3 beschriebene Kontroverse im Feld der politischen Bildung bezüglich des Potentials realen politischen Handelns für formale politische Bildungssettings verdeutlicht die Notwendigkeit, dass politische Aktionen von 
Schüler*innen immer auch den Anspruch verfolgen, politische Öffentlichkeit herzustellen und sich damit nicht außerhalb des demokratischen Diskursraumes bewegen, sondern diesen nachhaltig zu prägen versuchen. Frank Nonnenmacher weist daher darauf hin, dass bei politischen Aktionen von Schüler*innen, die in einem Verhältnis zu formalen Lernsettings wie dem Unterricht oder der Schule stehen, immer auch eine „,demokratische Öffentlichkeit hergestellt werden“ (Nonnenmacher 2010, S. 467) muss. Nonnenmacher betont darüber hinaus, dass diese Öffentlichkeit für eine mit der politischen Aktion ,,verbundene sichtbare Verantwortungsübernahme der ,Aktivisten " und für ein Klima der Diskursivität“" (Nonnenmacher 2010, S. 467) sorgt.

In dem folgenden Abschnitt dieser Arbeit werden die Ergebnisse der Analyse der Erfahrungsberichte aller interviewten Schüler*innen vorgestellt, die darauf schließen lassen, welchen Stellenwert die politische Öffentlichkeit für die Befragten einnimmt.

Das Herstellen von Öffentlichkeit ist ein Bedürfnis, dass allen befragten Schüler*innen wichtig ist und von Anfang an in den schulischen und außerschulischen Gruppen besondere Beachtung erfuhr. Beispielhaft dafür steht der Erfahrungsbericht von SOPHIE, die aus der Gründungsphase ihrer antirassistischen Jugendbewegung Folgendes berichtet:

Ich überlege gerade, wie das so war, am ersten Tag. Naja, wir sind dann irgendwie so zusammengesessen, einer hat Protokoll geschrieben (lacht), jeder hat mal gesagt, woher er so kommt, wie er heißt, was er macht, von welcher Schule er ist oder was er für eine Ausbildung macht oder sonst irgendwas, oder ob er überhaupt arbeitet (lacht). Und dann? Keine Ahnung. Dann hat es irgendwie angefangen, dass man gesagt hat, wie kriegt man Leute zusammen, dann haben sich Arbeitsgruppen gebildet, ein paar Leute, die Facebook betreuen, die irgendwelche Aktionen planen, die praktisch so PR-mäßig ein bisschen was machen. (SOPHIE: 209-216)

Die politisch aktiven Jugendlichen wollen Öffentlichkeit über verschiedene Strategien und Medien herstellen. Mehrere Interviewpartner*innen berichten davon, dass Öffentlichkeitsarbeit einen wesentlichen Teil ihrer Arbeit ausmacht. Sie verbinden damit unter anderem das Schreiben und Halten von politischen Reden bei Kundgebungen und Demonstrationen, die Organisation von öffentlichen Informationsveranstaltungen (RONJA: 1061-1080), das Entwerfen und Verteilen von Flyern (LAURENZ: 249-255), das Verfassen von Pressemitteilungen (RONJA: 1175-1178), die Arbeit über soziale Medien (SOPHIE: 209-216; 249254) und die Formulierung konkreter Forderungspapiere an die Politik (RONJA: 1021-1040). Aber auch Protestformen des zivilen Ungehorsams nutzen die Aktivist*innen, um Öffentlichkeit herzustellen. Ein Beispiel dafür ist die zeitweise 
Besetzung öffentlicher Plätze und damit die Aneignung des öffentlichen Raumes. FRANZISKA beschreibt das Ziel dieser Protestcamps damit, ihre politischen Forderungen in die Öffentlichkeit zu tragen, um möglichst viele Menschen zu erreichen.

\begin{abstract}
Da dachten wir, wir müssen auf jeden Fall was machen und wir müssen auch etwas machen, das die Aufmerksamkeit der Leute auf sich zieht. Wo wir auch was sagen können und wo wir auch gehört werden. Und deswegen haben wir beschlossen, dass wir einen öffentlichen Platz in der Stadt besetzen wollen und das haben wir auch gemacht. (FRANZISKA: 756-760)
\end{abstract}

Für FRANZISKA ist die erzielte Öffentlichkeit des von ihrer Gruppe organisierten Protestcamps ein bedeutendes Ziel, das durch die politische Arbeit erreicht wurde. Ihnen sei es gelungen, ihre politischen Forderungen in der Öffentlichkeit sichtbar zu machen und Aufmerksamkeit für das Thema zu erreichen. ,[W]ir haben in der Stadt mittlerweile auch einen gewissen Namen als Jugendbewegung, jeder kennt uns, jeder spricht uns an. “ (FRANZISKA: 832-833)

Mit Öffentlichkeit verbinden die Jugendlichen immer auch den Wunsch, die Themen, die sie bewegen, in ein öffentliches Bewusstsein zu rücken. Ihre Wahl der Aktionsform wird dabei auch von diesem Wunsch getrieben. So ist beispielsweise das zentrale Anliegen bei der Organisation eines Konzerts gegen Rassismus nicht das gemeinsame Verbringen eines schönen Abends mit Musik, sondern die Verbreitung der damit verbundenen Botschaft (JOHANNES: 424432). Öffentlichkeitsarbeit verbinden die Jugendlichen auch mit dem Bedürfnis, am öffentlichen Diskurs zu partizipieren. Diese Art der politischen Artikulation und das dafür notwendige Selbstvertrauen entwickeln sie im Prozess des politischen Handelns in der öffentlichen Sphäre.

Oder wo ich mir sehr unsicher wäre: Reden halten. Also, am Anfang war ich noch, also, ich kann vor Menschen sprechen, aber wenn ich auf der Bühne stehe, bin ich doch ziemlich aufgeregt und kann nicht so gut Reden schreiben und das hat sich total gebessert. Und mittlerweile, bei der Gegen-Erdgas-Demo habe ich vor 800 Menschen gesprochen. Und das ist sowas, was ich sonst nie gemacht hätte. Und das sind so Sachen, die echt cool sind, finde ich. Also, in dieser Hinsicht lerne ich sehr viel, glaube ich. (RONJA: 1169-1175)

Das Bedürfnis nach Öffentlichkeit schafft Legitimation für reales politisches Handeln der Schüler*innen, denn es entspricht dem Selbstverständnis der Idee einer republikanisch-demokratischen Gesellschaft. 
Für die politische Bildung ist aber nicht nur von Bedeutung, dass die Schüler*innen mit ihren politischen Aktionen Teil des öffentlichen Diskurses sind. Vielmehr ist für die politikdidaktische Forschung relevant, welche Lernprozesse im Feld der Handlungskompetenz sich aus dem realen politischen Handeln entwickeln. Fast beiläufig, aber dabei für die Subjekte nicht weniger wichtig, erscheinen dabei Organisationsfähigkeiten. Diese ließen sich aus dem politikdidaktischen Diskurs zur Handlungskompetenz kaum ableiten (siehe Abschnitt 2.2.2) und, wenn überhaupt, eher in nicht fachspezifische und übergreifende Kompetenzfelder der methodischen Fähigkeiten zuordnen. Im Prozess der Rekonstruktion individueller Lernerfahrung in der politischen Aktion kann im Zuge der induktiven Analyse der Erfahrungsberichte konstatiert werden, dass die politische (Selbst)Organisation einen hohen Stellenwert im Lernprozess der aktiven Schüler*innen einnimmt. Exemplarisch sollen Erkenntnisse in diesem Lernfeld im Folgenden dargestellt werden.

Unabhängig von den Fähigkeiten, die unmittelbar mit politischen Lernprozessen verbunden werden, schafft das politische Engagement Erfahrungsräume für die politische (Selbst)Organisation und die Reflexion über politische Prozesse. Alle interviewten Schüler*innen bringen Lernerfahrungen im Zuge von Organisationsprozessen immer auch in den Zusammenhang mit notwendigen Grundkompetenzen, die ihnen den Alltag erleichtern.

Ja, also, es gibt verschiedene Sachen, die mir mein Leben leichter machen, dadurch, dass ich mich engagiere. Zum einen ist es halt wirklich so, dass man sehr viel dadurch lernt, unterbewusst einfach, weil man zum Beispiel lernt, sich eine eigene Meinung zu bilden oder sich selbst zu reflektieren oder was zu organisieren. Weil, du kannst dich nicht für irgendwas engagieren, ohne dass du dafür irgendwie auch nur ein kleines bisschen organisiert hast. Also, man muss immer irgendwas organisieren. (LEA: 837843)

An einem Fallbeispiel beschreibt RONJA ganz konkret, welche Kompetenzen zur Strukturierung ihrer Arbeit sie durch ihr politischen Engagement entwickelt hat.

Dann haben wir uns auf ein Thema spezialisiert und haben dann den Plan dafür erstellt, wie viele Tage wir zur Verfügung haben und dann zugeteilt, wer sich um was kümmert und ob wir Hilfe anfordern können. (RONJA: 286-288)

RONJA betont den Wert dieses Organisationswissens. Die damit verbundenen Fähigkeiten, wie das Koordinieren von Projekten und die Zusammenarbeit mehrerer Menschen zu planen, hat sie durch ihr politisches Engagement entwickelt. Diesen Zusammenhang betont sie explizit: 
Im Allgemeinen würde ich schon sagen, dass das aufjeden Fall lehrt, Projekte selber zu machen. Aber für mich ist das besonders wichtig, weil ich das auch sehr gerne mache, zu organisieren. Wie kann ich eine Gruppe am schnellsten zum Ziel führen, quasi und so, dass alle daran beteiligt sind und nicht, dass ich dann die ganze Arbeit mache. Und dass ich das besser koordinieren kann, weil sonst arbeite ich mich dann einfach tot dran, sodass ich das dann auch gemerkt habe, weil ich ja eben noch gesagt habe, es braucht diesen einen Leitenden, wenn so viel Klausurenphase ist zum Beispiel. Und darum habe ich mich gar nicht so sehr mit dem einzelnen Thema beschäftigt, sondern eher, das zu koordinieren. Aber dabei habe ich auf jeden Fall eine Menge gelernt. (RONJA: 357-367)

Die Organisationsfähigkeit ist aber immer auch eng verbunden mit politischen Lernerfahrungen. Dies wird besonders deutlich, wenn die interviewten Schüler*innen die Bedeutung von kooperativen Prozessen zur Findung inhaltlicher Linien oder Projektideen hervorheben. Dabei lernen sie nicht nur, sich mit politischen Themen zu befassen, sondern auch wie sich politische Positionen in einer Gruppe entwickeln lassen und welche Grundregeln für diesen komplexen politischen Prozess notwendig sind. So betonen die interviewten Personen immer wieder die Notwendigkeit eines politischen Selbstverständnisses in der Gruppe, mit dem sie auch rote Linien formulieren. Dazu gehören Sexismus, Homophobie, Rassismus und Gewalt (FRANZISKA: 606-610).

Vor allem das selbstbestimmte und selbstorganisierte politische Handeln ermöglicht den jungen Menschen, praktische Erfahrungen in der Organisation politischer Gruppen zu sammeln und dabei Prozesse zu erfahren und zu reflektieren, die in formalen Lernsettings, wie dem Unterricht, der schulischen Projektarbeit oder in außerschulischen Kontexten wie Vereinsarbeit, Verbänden oder der Parteijugend bereits etabliert sind. Wie RONJA haben sich die meisten Schüler*innen im Zuge ihres politischen Engagements erstmals mit formalen Merkmalen der Organisation in einer politischen Gruppe befasst. „,Man muss da auch Protokolle und so schreiben, was ja alles nicht so einfach ist." (RONJA: 634-635) Die Schüler*innen lernen Prinzipien politischer Selbstorganisation in Gruppen, wie beispielsweise Arbeitsteilung:

Und es ist jetzt mittlerweile auch einfach so, wenn jetzt irgendwer etwas auf Facebook posten will, dann wird natürlich nicht jeder vorher gefragt, sondern dann hat man diese Gruppe, die sich darum kümmert. Und wenn die sagen, dass wir das jetzt so posten, dann wird das so gepostet. Also, das ist ja auch Unsinn, wenn man dann alles immer so ins kleinste Detail absprechen müsste. (SOPHIE: 249-254)

Diese Prozesse der Bildung von Arbeitsgruppen, das Bestimmen von Tagesordnung und die Festlegung eines Selbstverständnisses der Gruppe empfinden die 
Jugendlichen als politischen und demokratischen Erfahrungsraum. LAURENZ beschreibt das wie folgt:

Also, erst mal bin ich ja in einer Gruppe organsiert, die auch wöchentlich Plena abhält. Damit fängt es letztendlich an, dass ich wöchentlich mich an politischen, an demokratischen Entscheidungsprozessen beteilige. Wir setzen uns, letztendlich sind das ja schon Rätestrukturen. Wir setzen uns in einem Raum zusammen, wir sind 20 Leute oder so. Und dann wird eine Tagesordnung durchgegangen und zu Fragen, bei denen es Kontroversen geben könnte, wird eine Diskussion aufgemacht. Jeder kann etwas dazu sagen. Die Argumente werden von jedem Einzelnen gegeneinander abgewogen und schlussendlich wird abgestimmt. (LAURENZ: 242-249)

Wenngleich diese politischen Organisationsfähigkeiten kein Alleinstellungsmerkmal politischer Bildungsprozesse sind, so stellen sie einen bedeutenden Teil der Erfahrungswelt in selbstbestimmten politischen Partizipationsprozessen dar. Die Bedeutung dieser Erfahrung wird deutlich, wenn die Jugendlichen betonen, dass die meiste Zeit bei den Gruppentreffen für organisatorische Fragen aufgebracht wird (SOPHIE: 266-275).

Vor allem für die Jugendlichen, die sich in autonomen Jugendgruppen außerhalb der Schule organisiert haben, ist die Auseinandersetzung mit demokratischen Prinzipien der Entscheidungsfindung innerhalb der Gruppe ein bedeutendes Anliegen. Es scheint gar ein Gegenpol zu den antidemokratischen Erfahrungen aus schulischen Lernkontexten zu sein. Sie nutzen und reflektieren dabei Instrumentarien wie (doppelt)quotierte Redelisten oder Entscheidungsprinzipien wie das Mehrheits- (FRANZISKA: 624-641) oder das Konsensprinzip (SOPHIE: 236249). Und auch für die sich daran anschließenden politischen Aktionen wird in der Regel ein Aktionskonsens beschlossen. LAURENZ erklärt, wie die Gruppe vor Kundgebungen und Demonstrationen einen Aktionskonsens entwickelt und warum er diesen für elementar hält. LAURENZ verweist darüber hinaus auf die Bedeutung von Bezugsgruppen und wie die Gruppe versucht, über diese Instrumente die verschiedenen Bedürfnisse der Aktivist*innen zu berücksichtigen.

Das bedeutet, dass wenn wir (..) zum Beispiel sagen: Wir erklären uns mit allen Aktionsformen gegen die AfD solidarisch. Das bedeutet nicht, dass wir alle selbst ausführen, aber dass wir nicht dastehen werden und diejenigen, die sie ausführen, zum Beispiel dann verurteilen. Um jetzt irgendein Beispiel zu nennen. Und es werden ja Bezugsgruppen festgelegt. Das bedeutet, dass man sagen kann: Okay, ich möchte eher aktiv an der Demonstration teilnehmen. Oder: Ich möchte aktiv-passiv sein, das Mittelding. Oder eben passiv. Je nachdem, kann man sich mit anderen Leuten in einer Bezugsgruppe zusammenfinden. Und dann eben auch so in der Demonstration eingliedern, gruppieren, dass man eben auch auf, ja, selbst das irgendwie entscheiden kann. Was 
wir nicht machen, ist, keinen Aktionskonsens fassen, was manche andere Gruppen machen. Manche Gruppen sagen beispielsweise, ein Aktionskonsens an sich sei autoritär. Der Meinung bin ich nicht, weil der demokratisch festgelegt wird und jedem ein Freiraum gelassen wird. Und diese Gruppen sind dann teilweise der Meinung, dass eben keine Gruppen gebildet werden sollten, sondern ganz individuell jeder für sich entscheiden sollte. Und ich denke, dass das für eine Demonstration, für eine funktionierende Demonstration vollkommen kontraproduktiv ist, weil alles in sich greifen muss wie ein Zahnrad. Und alles muss funktionieren. Und dafür ist ein Konsens, meiner Meinung nach, dringend nötig. (LAURENZ: 685-703)

Neben Erkenntnissen zu den Prinzipien der politischen Selbstorganisation haben sich die Jugendlichen auch ganz praxisnah mit den Herausforderungen, Wirkungsweisen und organisatorischen Merkmalen verschiedener politischer Aktionsformen befasst. Das erscheint zunächst evident, ist aber gerade deswegen von Bedeutung, weil grundlegende Informationen dazu, welche Voraussetzung es gibt für die verschiedenen Ausdrucksformen konventioneller und unkonventioneller politischer Partizipation, in der Schule eher eine untergeordnete Rolle spielen.

Die Jugendlichen berichten, dass das politische Engagement sie vor allem dazu befähigt hat, verschiedene Partizipationsformen umzusetzen und ihre Wirkungskraft zu reflektieren (hierzu ausführlich in Abschnitt 4.3.4.5). Das gilt im Übrigen sowohl für jene, die für ihre politischen Aktionen Freiräume in der Schule erstritten haben, als auch für die Jugendlichen, die sich im außerschulischen Raum politisch engagieren. JOHANNES, der sich in einer selbstorganisierten AntiRassismus-AG einbringt, erklärt, dass er durch die Arbeit in der Gruppe viel darüber gelernt habe, wie eine politische Veranstaltung organisiert werde und was dabei zu beachten sei. „Ich weiß, wie es ist, Projekte durchzusetzen. Und vor allem hat es mir persönlich auch viel Unsicherheit insgesamt weggenommen, weil ich halt weiß, wie ich etwas organisieren kann und vor allem, dass es gar nicht so schwer ist. “ (JOHANNES: 567-571) Dabei werden grundsätzliche Organisationskompetenzen durchaus eng verknüpft mit politischen Bildungsprozessen. KATJA beschreibt beispielsweise die Herausforderung, für einen Aktionstag die Referent*innen auszuwählen.

Wir haben uns zusammengesetzt und gesagt, die und die Leute könnten wir anschreiben oder irgendwie keine Ahnung, fragen, ob die nicht Lust haben, einen Workshop zu machen. Da gab es auch so ein bisschen Differenzen, wen wir da so nehmen und wen nicht. Und dann irgendwann haben wir halt alle Leute angeschrieben und dann war es total schnell so, dass wir gemerkt haben, wir haben super viele Workshops, aber nur fünf Tage Zeit. Und dann haben wir uns halt zusammengesetzt und haben überlegt, das und das sortieren wir aus und das nicht. Und da haben die Lehrer auch ein bisschen ausgenutzt, dass sie ein bisschen mehr Mitspracherecht haben als wir und auch gesagt: 
Das fände ich jetzt gut. Und wir haben gesagt: Aber echt nicht. Ja, aber das fänden wir jetzt gut. Und dann war es halt so. Also, auch nicht bei allen Sachen, aber bei einem Workshop war es sehr krass, sodass wir das eigentlich überhaupt nicht wollten und sie gesagt haben: Doch, das ist eine tolle Chance. Im Nachhinein fanden wir den alle blöd, die Lehrer auch (lacht). (KATJA: 512-525)

Wenngleich die Schüler*innen in der Regel sehr froh über die Unterstützung von Lehrkräften sind und auch immer wieder betonen, dass es mit den Lehrkräften, die sich in den selbstorganisierten Arbeitsgemeinschaften einbringen, kaum Rollenkonflikte gibt, zeigt dieser Bericht von KATJA, wie wichtig es ist, dass sich Lehrkräfte ihrer besonderen pädagogischen Rolle in selbstbestimmten politischen Aktionsformen von Schüler*innen bewusst werden müssen, um nicht - wenngleich auch ungewollt - Frustrationserfahrungen zu verursachen. Dennoch, diese Form der Einmischung hat auch zu einem Lernprozess geführt, den die Schüler*innen intensiv aufgearbeitet haben. Die Frustrationserfahrung ist in jedem Fall höher, wenn Lehrkräfte die Unterstützung für politische Aktionen der Schüler*innen vollständig verweigern. Das wird aus den Erfahrungsberichten von RONJA (964-999) deutlich, die im Kontext der „Fridays for Future“-Bewegung eine Nachhaltigkeitswoche an ihrer Schule organisieren wollte und dabei keine Unterstützung von ihren Lehrkräften erfahren hat.

Auch mit unkonventionellen Beteiligungsformen wie Demonstrationen setzen sich die Schüler*innen auseinander. Sie lernen viel darüber, was es bedeutet, eine Demonstration und eine politische Kundgebung vorzubereiten und durchzuführen.

\begin{abstract}
Also, es war sehr spontan die Aktion. Am Dienstag haben wir die dann gemacht, die Demo. Und an dem Donnerstagnachmittag haben wir entschieden, dass wir das machen wollen. Also haben wir dann den ganzen Freitag rumtelefoniert, auch wir Schüler selber. Wir haben sehr viel selber gemacht. Das Einzige was wir gemacht haben, wir haben halt die Lehrer und die Schulleitung immer gefragt, ob das in Ordnung ist, und haben dann nach Telefonnummern gefragt oder so. Natürlich hat die Schulleitung sich viel Zeit genommen und hat sich über die rechtlichen Sachen informiert, was wir dürfen und was nicht. Aber das meiste haben wir Schüler selbst ausgearbeitet. Wir als SV haben rumtelefoniert. Ja. (MICHAEL: 196-205)
\end{abstract}

Konkret beschreibt RONJA, welche Fragen im Vorfeld zu klären waren: „Und dann haben wir die groben Pläne gemacht, wo lang wir laufen wollen, Musik, wer die Reden hält, wer die Presse macht, Ordner, was unser Ziel ist, wer die Plakate macht, wen wir noch ansprechen. " (RONJA: 723-726)

Sie erleben in der ganz konkreten Praxis auch die Hürden in der Ausübung von Grundrechten, wie beispielsweise des Rechts auf Versammlung (GG, Artikel 8, Abs. 1). 
Genau, dann haben wir uns diese verschiedenen Themen überlegt, was jetzt alles noch $z$ organisieren ist. Haben das dann ziemlich schnell gemacht. Marcel hat momentan bislang die ganzen Demos angemeldet in der Stadt. Kann er jetzt leider nicht mehr machen. Darum mache ich jetzt im Grunde quasi dieses Anmelden, beziehungsweise noch eine Freundin aus der Jugendbewegung. Weil eigentlich, glaube ich, In unserer Stadt ist das ein bisschen komisch. Bei uns darf man mit 16 noch anmelden. In anderen Städten darf man es erst ab 18. Und ich weiß, dass andere Ortsgruppen von Fridays For Future da Probleme mit haben, jemanden 18-Jährigen zu finden, der die Demos anmeldet. Aber das ist bei uns irgendwie gar kein Problem gewesen bislang. (RONJA: 740-749)

Aus den Interviews mit den Schüler*innen lässt sich ableiten, dass vermeintlich fachunspezifische Kompetenzen wie die Organisationsfähigkeit im hohen Maße politisch sind und vielfältige Lernanlässe schaffen.

Der Politikdidaktiker Peter Henkenborg weist darauf hin, dass das DemokratieLernen auch eine ,Auseinandersetzung um , billigenswerte ( (lobenswerte) Charaktereigenschaften, um die Frage, wie wünscht man, dass alle handeln oder sind“"“ (Henkenborg 2005, 303 f.) erfordere. Henkenborg betont, dass diese Tugenden den Kern demokratischer Sittlichkeit darstellten. Damit verbunden sind Fähigkeiten wie Kommunikations-, Konflikt-, Konsens- und Kritikfähigkeit. Im Kontext der Handlungskompetenz steht für die vorliegende Untersuchung vor allem die Konfliktfähigkeit im Fokus - die Erkenntnisse dazu werden im späteren Verlauf dieses Kapitels vorgestellt (4.3.4.4).

Im folgenden Abschnitt sollen aber zunächst soziale Kompetenzen in den Mittelpunkt gerückt werden. Dabei werden Ergebnisse der Analyse des empirischen Materials vorgestellt, die Rückschlüsse darauf zulassen, inwiefern das Mitwirken an selbstbestimmten politischen Aktionen auch auf die Entwicklung sozialer Kompetenzen wirkt. Die induktive Analyse des Interviewmaterials ergab, dass vor allem drei Elemente als wertvolle Lernanlässe rekonstruiert werden können. Der soziale Umgang in der Gruppe, insbesondere in der Auseinandersetzung mit Frustrationserfahrungen, das Erlernen kooperativer Führungskompetenzen und der Wissenstransfer innerhalb der Gruppe.

Die Jugendlichen schätzen den Wert der Gruppe als wichtigen sozialen Raum ein, deren Einfluss weit über das bloße Verfolgen gemeinsamer politischer Ziele hinausgeht. Sie bezeichnen die Gruppe als einen Schutzraum, der ihnen persönliche Selbstentfaltung ermöglicht, ohne Angst vor Diskriminierung haben zu müssen. Der wertschätzende und respektvolle Umgang, frei von Rassismus, Sexismus und anderen Formen der Ausgrenzung, ist für die politisch aktiven Jugendlichen ein Wesenskern ihrer politischen Arbeit. Sie betonen, dass sie sich innerhalb der Gruppe einen angstfreien Lernraum geschaffen haben, der sich 
damit klar von anderen Erfahrungsräumen des politischen Lernens, wie der Schule und dem Unterricht, unterscheidet. Eindrucksvoll wird dies an den Aussagen von EMMA deutlich:

Ja (...) ich bin erst mal dahingegangen, und ich war natürlich erst mal überrascht, wie es abläuft, also das ist halt, der Respekt der einander entgegengebracht wird. Es ist ein ganz anderer sozialer Raum als man ihn beispielsweise in der Schule hat oder im Freundeskreis. Sowas hatte ich auch vorher noch nie kennengelernt. Es wird sehr viel Wert darauf gelegt, dass jeder gleichwertig respektiert wird (..) Egal wie, ob er jetzt stärker gebildet ist, weniger gebildet ist, ob er ansonsten sozial akzeptiert ist oder nicht, man diskutiert einfach mit jedem auf der gleichen Ebene, ohne dass darüber geurteilt wird; im Endeffekt, ohne dass man sich schlecht fühlen muss, wenn man irgendwie etwas weniger weiß als der, der jetzt irgendwie schon 22 oder 24 ist. (EMMA: 221-231)

Weiter im Verlauf des Interviews konkretisiert EMMA diese Erfahrungen und verweist darauf, dass die politische Bewegung sich durch Hierarchiefreiheit, Akzeptanz und Respekt auszeichnet.

Im normalen Schulraum, im Freundeskreis da gibt es ja immer gewisse soziale Strukturen, also da gibt es Hierarchien, da gibt es einen Coolen, da gibt es den, der sich immer ein bisschen blöd stellt, da gibt's den einen, der halt immer ein bisschen ausgegrenzt wird, der immer ein bisschen geneckt wird, der nur so das dritte Rad am Wagen ist. Also, da gibt es halt ganz verschiedene Kompetenzen in diesen sozialen Strukturen. Und es ist immer auch ein kleiner Kampf, dass man nicht nach unten in der Hierarchie rutscht, würde ich sagen. Also für mich war es das jedenfalls immer und das war da halt überhaupt nicht, also ist halt überhaupt nicht gegeben in der politischen Gruppe. Der 24-Jährige ist sich jetzt nicht zu schade irgendwie, mal mit einer 15-Jährigen in eine Diskussion zu treten, und behandelt die dann auch nicht von oben herab. Und es gibt auch nicht dieses typische „hin- und herdissen“ irgendwie so: Ich bin cooler als $d u$. Und es gibt natürlich auch viele Menschen, die da sind, die auch ein bisschen Außenseiter der Gesellschaft sind, würde ich sagen, und die oft nicht so den Anschluss finden. Und die dann aber da halt akzeptiert werden, so wie sie sind, und nicht verspottet werden, ausgelacht werden, kritisiert werden die ganze Zeit, selbst wenn sie Makel haben. Das ist halt so ein zusammen gegenseitiges Lernen und Wachsen aneinander. Also ich weiß nicht, es ist auf jeden Fall schwer zu beschreiben. (EMMA: 235-254)

Der in den Gruppen offenbar vorherrschende Konsens eines wertschätzenden Umgangs miteinander ermöglicht auch einen besseren Umgang mit Frustrationserfahrungen. FRANZISKA beschreibt, wie ihre Gruppe mit diesen Erfahrungen umgeht und welche Fragen sie sich stellen, um diese aufzuarbeiten. 
Und es gab auch immer im politischen Umfeld die Möglichkeit, so etwas zu reflektieren, dass man eben dann darüber geredet hat. Wie hat sich jeder dabei gefühlt? Woran hat das gelegen? Was hätte man besser machen können? Wie gehen wir in Zukunft mit dieser Erfahrung um? Und so wird eigentlich jede direkte Frustration dann doch wieder zu etwas, das einen dann auch weiterbringt. Denn es kann natürlich nicht immer alles funktionieren. Und das ist ja nicht nur im politischen Bereich so, sondern überall im Leben, dass man nicht alles schafft, was man möchte. (FRANZISKA: 561-570)

Diese Art des sozialen Umgangs und der Hierarchiefreiheit innerhalb der politischen Gruppe ist auch eng verbunden mit dem Selbstverständnis der Jugendlichen, einen möglichst hohen Wissenstransfer zwischen erfahreneren und weniger erfahrenen Aktivist*innen $\mathrm{zu}$ erreichen, um neben allgemeiner Hierarchie auch Wissenshierarchie aufzubrechen. JAN verweist beispielsweise darauf, wie sich politische Jugendgruppen untereinander austauschen und auf dem Wissen und den Erfahrungen anderer Gruppen aufbauen wollen (JAN: 345-352), SOPHIE erklärt dieses Phänomen an folgendem Beispiel:

\begin{abstract}
$J a,(. .4 \ldots)$ das war eigentlich, ich sage jetzt mal, irgendwo angenehm, aber es war auch ziemlich zäh natürlich, weil dann natürlich viel diskutiert worden ist. Es gab dann schon Leute, die so etwas vielleicht schon länger machen und dann natürlich auch mehr Ahnung hatten. Also, ich bin dahin gekommen und dann hieß es: Okay, was machen wir? Konsensprinzip! Und ich sitze da und denke mir: Okay, toll, erklärt mir mal jemand dieses Konsensprinzip? Also, ich war nicht die Einzige, die das nicht wusste, aber das ist ja dann auch gleich erklärt worden. Es ist nicht so, also würde ich sagen, dass es so eine Megahierarchie gibt von Leuten, die das schon länger machen, und Leuten, die jetzt irgendwie neu dazu sind. Das ist schon gleich. Man merkt, wer mehr Erfahrung hat. Aber die lassen es nicht raushängen oder so etwas, überhaupt nicht. (SOPHIE: 284-294)
\end{abstract}

Die Ausführungen von EMMA und FRANZISKA scheinen auf den ersten Blick im Widerspruch zu RONJAS Ansicht zu stehen, die die Notwendigkeit von Strukturen und Führungskraft in der Gruppe betont. RONJA verweist darauf, dass es für die Arbeit in politischen Gruppen immer auch einer Person bedürfe, die die Leitung und die Verantwortung übernimmt.

Aber tatsächlich (...), da merke ich dann aus unserer Gruppe besonders, dass es dann eine leitende Person braucht. Also, das bin ich an dieser Stelle, im Moment zumindest. Und wenn sich da - das beruft sich dann viel auf diese Person - und ich habe das Gefühl, dass es das in Schüler*innengruppen sehr braucht, weil alle natürlich mit ihren Sachen viel zu tun haben. Und da braucht es jemanden, der das anleitet. Und wenn es dann kein Lehrer macht oder ein Pädagoge oder irgendwer, dann muss halt eine Person übernehmen. Das bin ich im Moment. (RONJA: 251-258) 
RONJA verbindet die Notwendigkeit, Führungskompetenzen zu entwickeln damit, Mitstreiter*innen zu motivieren und weiterhin für die politische Arbeit zu begeistern (RONJA: 383-396). Auch JAN reflektiert seine Rolle in der politischen Gruppe und stellt für sich fest, dass er vor allem die sozialen Kompetenzen des Führens, Anleitens, aber eben auch des Moderierens und Motivierens weiterentwickeln möchte. „Ich müsste vielleicht mal so einen Moderationskurs machen (lacht). Ich habe halt immer total viele Ideen und bringe die dann auch immer sofort ein. Und dann kommt es eben an solchen Stellen schnell zu der Situation, dass dann alle nicken und dann die Eigeninitiative so von den anderen Leuten genommen wird." (JAN: 569-574) Diese Fähigkeit zur Reflexion der eigenen Sozialkompetenz ist unmittelbar auch verbunden mit den Erfahrungen aus der selbstbestimmten politischen Partizipation, denn sie ist eng gekoppelt an die Erkenntnis, dass politische Arbeit alleine nicht zu bewältigen ist und die politischen Ziele auf diesem Weg nicht erreicht werden können.

Soziale Bildungsprozesse werden nicht nur gruppenintern angeregt. Die Schüler*innen sind auch vielfältig sozial engagiert. Wenngleich ein wesentlicher Faktor für die Auswahl der Gruppen war, dass sie explizit politisch aktiv sind, so lassen sich soziales Engagement, Empowermentarbeit und politische Partizipation kaum losgelöst voneinander betrachten (siehe Abschnitt 2.1.2 „Dialektik der Partizipation“). Daher werden im folgenden Kapitel Bildungserfahrungen von Jugendlichen rekonstruiert, die im Sinne eines sozialen Engagements einzustufen sind.

\subsubsection{Soziales Engagement}

Wie im Kapitel zu den theoretischen und empirischen Grundlagen bereits beschrieben, lassen sich soziales Engagement und politische Partizipation nicht trennscharf unterscheiden. Vor allem ist empirisch bisher noch unzureichend erforscht, inwiefern aus sozialem Engagement, das gesellschaftlich eine höhere Anerkennung genießt als selbstbestimmte politische Partizipation, politisches Lernen oder gar politisches Handeln erwachsen kann (u.a. Reinhardt 2011, 2013; Wohnig 2015, 2017). Dennoch: Für die vorliegende Studie kann konstatiert werden, dass das politische Engagement der befragten Schüler*innen zumeist seinen Ursprung im sozialen Engagement hat. Das ist dabei besonders vielfältig und reicht von Pfandsammelaktionen für den guten Zweck (JOHANNES: 252-258), über Patenschaften für Geflüchtete (ESRA: 673-679) bis hin zur Neugestaltung der Mensa (MICHAEL: 95-98). Oftmals sind diese Formen des sozialen Engagements verbunden mit den ersten Erfahrungen in der Schüler*innenselbstverwaltung. „Die SV engagiert sich sozial an der Schule, damit die Schüler auch glücklich sind. Damit die Schüler auch glücklich sind, verändern 
wir auch ein bisschen was hier an der Schule, äußerlich, oder so." (MICHAEL: 80-81) MICHAEL definiert die Aufgabe der Schüler*innenvertretung mit dem Selbstverständnis, die Wünsche der Schüler*innen zu erfüllen (MICHAEL: 8487). Sie wird aber auch vonseiten der Schulleitung instrumentalisiert, um Geld für geplante Anschaffungen zu akquirieren.

\begin{abstract}
Wir unterhalten uns auch oft mit der Schulleitung, ob das in Ordnung ist, was sie davon hält. Oder auch die Schulleitung gibt uns manchmal Problemstellungen. Zum Beispiel hatten wir jetzt erst: Die Schulleitung wollte eine Hängematte kaufen und wusste nicht, wie sie die finanzieren soll. Und dann haben wir uns überlegt, wie wir das finanzieren können als SV, als Schule und haben dann viele Vorschläge gehabt. Und haben uns dann letztendlich auf einen geeinigt, sodass wir als SV einen Beitrag bezahlt haben, den wir durch unseren Kaffeeautomaten erwirtschaftet haben. Und so Sachen besprechen wir halt immer. (MICHAEL: 87-95)
\end{abstract}

Diese Erzählung steht beispielhaft für verschiedene Berichte der Schüler*innen, die darauf hindeuten, dass die SV instrumentalisiert wird und ihr Engagement sich nicht selten an den Interessen der Schulleitung oder der Lehrkräfte zu orientieren hat. Dies führt zu Frustrationserfahrungen, die bei den Schüler*innen den Eindruck einer Alibi-Partizipation verstärken (Anerkennungs- und Frustrationserfahrungen werden ausführlich in Abschnitt 4.3.5 „Politisches Selbstkonzept“ rekonstruiert).

Die Jugendlichen haben sich daher oftmals Räume losgelöst von den institutionalisierten Strukturen der Schüler*innenmitwirkung gesucht. Vor allem das Jahr 2015 und die enormen Bewegungen von Geflüchteten nach Deutschland hat viele neue Möglichkeiten des sozialen Engagements geschaffen. So war auch KATJAs erste sozialpolitische Aktion verbunden mit dem Engagement für Geflüchtete in ihrer Stadt. Beispielsweise hat sie mit Freund*innen zusammen Kleidung gesammelt. „Und wir haben angefangen Aktionen zu machen und haben Klamotten gesammelt für Geflüchtetenheime und uns hier in der Stadt stark für die Geflüchteten eingesetzt. " (KATJA: 253-258) Auch RONJA und ESRA haben sich gemeinsam mit ihren Mitstreiter*innen für Geflüchtete in ihrer Stadt beziehungsweise an ihrer Schule engagiert. RONJA beschreibt das wie folgt:

Ja. Also, da ging es viel darum, wie man zum Beispiel die Geflüchteten aus Anklam mit uns verbinden kann. Wir haben schon einmal einen Musikabend gemacht. Dazu haben wir die eingeladen und auch gegrillt und zusammen Musik gemacht, getanzt und verschiedene Kulttänze ausgetauscht. Und danach dachten wir uns, dass wir so etwas noch einmal machen wollen und hatten eine Sportveranstaltung und haben Basketball gespielt. (RONJA: 272-277) 
Dieses soziale Engagement hat die Schüler*innen aber auch politisiert. Aus der Geflüchtetenhilfe sind Jugendinitiativen und Arbeitsgemeinschaften gegen Rassismus entstanden und neben dem sozialen Engagement für Geflüchtete vor Ort entwickelten sich immer häufiger auch politische Solidaritätsaktionen für Geflüchtete im Allgemeinen.

Dann kamen irgendwie so Sachen dazu, dass Leute ankamen und gesagt haben: Da soll es jetzt irgendwie eine Soli-Aktion für Geflüchtete geben, wir wollen da so in drei Wochen anfangen. Habt ihr nicht Lust da mitzumachen? Und dann haben wir diese Soli-Aktion gemacht. Von da ging dann relativ viel aus. Das war dann ein bisschen so Sammelpunkt, wo sich dann alle auch jeden zweiten Tag oder so getroffen haben. Und dann hatten wir halt sehr viel Kontakt zu Geflüchteten und dann haben die irgendwie erzählt: >> Unsere Kumpels sollen abgeschoben werden. $<<($ KATJA: 566-572)

Unter anderem aus diesen Erfahrungen sind verschiedene legale und illegale politische Aktions- und Organisationsformen politischer Partizipation entstanden, die im Fokus dieser Arbeit stehen und im Folgenden genauer dargestellt werden.

\subsubsection{Formen politischer Partizipation}

Um die politischen Bildungsprozesse im Zuge des selbstbestimmten politischen Engagements der Schüler*innen rekonstruieren zu können, ist es wichtig, die verschiedenen Aktionsformen zu kennen und dabei auch die Bedeutungen der jeweiligen Aktionsformen für die Jugendlichen zu verstehen. Im Folgenden werden daher die verschiedenen Formen politischer Partizipation kurz vorgestellt und mit exemplarischen Erfahrungsberichten der Schüler*innen verknüpft. Die Aktionsformen sind dabei in legale und illegale Partizipation untergliedert, wobei die legalen Aktionen noch mal in schulische und außerschulische Formen der politischen Partizipation unterteilt werden. Alle interviewten Personen haben Erfahrungen mit unterschiedlichen Partizipationsformen gesammelt.

\section{Schüler*innenvertretung}

Für die meisten Schüler*innen war das Engagement in der Schüler*innenvertretung die erste Partizipationserfahrung, wobei diese zumeist eher mit Frustrationserfahrungen verbunden waren. Sie sind überzeugt, dass die Vertretung der Schüler*innen häufig eher eine Alibi-Funktion einnimmt und sie und ihre Interessen kaum berücksichtig werden. Sie verbinden die Arbeit mit dem Gefühl, ausgenutzt (SOPHIE: 444-457) oder ignoriert (JOHANNES: 258-263) zu werden. Vor allem die Erfahrung in der SV hat vielen politisch aktiven Jugendlichen den Eindruck vermittelt, es würde sich bei der Schule 
ausschließlich um einen „Zweckverband zum Lernen“ (RONJA: 213) handeln. Ausführlich werden die auch mit der SV verbundenen Frustrations- und Anerkennungserfahrungen der Schüler*innen in Kapitel mit 4.3.5 „Politisches Selbstkonzept" zusammengeführt.

Wenngleich die SV grundsätzlich nicht mit einem politischen Mandat ausgestattet ist, kann sie aber durchaus der Anstoß für politisches Handeln und die Entwicklung selbstbestimmter politischer Projektarbeit an der Schule sein. Ein Beispiel dafür ist eine Antirassismusdemonstration, die von der SV einer Schule organisiert wurde. Nach Übergriffen auf ein Geflüchtetenheim und der medialen Berichterstattung darüber wollten sich die Schüler*innen gemeinsam mit ihrem Verbindungslehrer engagieren, weil sie ,unbedingt tätig werden wollten, wegen dem Fall in Rostock" (MICHAEL: 171-178). Sie haben sich dann dafür entschieden, eine Demonstration zu organisieren, an der sich nahezu alle Lehrkräfte und Schüler*innen beteiligt haben.

Es sind aber nicht immer konkrete politische Anlässe, die das Potential der SV für politisches Handeln offenbaren. Die Erfahrungsberichte anderer Jugendlicher lassen darauf schließen, dass auch Initiativen wie das Bündnis „Schule ohne Rassismus - Schule mit Courage" den Wunsch nach politischer Teilhabe bei den Schüler*innen auslösen kann. Mehrere Schüler*innen berichteten, dass ihre Arbeitsgemeinschaften ursprünglich aus einer Idee der SV hervorgingen, sich an dem Bündnis zu beteiligen. Aus dieser Idee wurde dann eine eigenständige, selbstorganisierte Initiative, die sich von der Arbeit der SV emanzipierte (RONJA: 229-234). Dieser Emanzipationsprozess ist für die Gruppe und die Schüler*innen wichtig, denn die SV verbinden sie zumeist mit unpolitischen, eher ordnungspolitischen Aufgaben in der Verwaltung der Schule. „Dann bin ich noch in der SV. Da ist es halt irgendwie Schulpolitik eher, als dass es Politik aus dem Bezirk ist oder was ist, was im ganzen Land relevant ist. “ (LEA: 740-742)

\section{Selbstorganisierte Arbeitsgemeinschaften und Initiativen}

Mehrere Schüler*innen, die zuerst Erfahrungen in der SV gesammelt haben, engagieren sich anschließend oder parallel an ihren Schulen in selbstorganisierten Arbeitsgemeinschaften und Projektgruppen. Alle Schüler*innen, die in diesen emanzipatorischen Gruppen in der Schule politisch aktiv werden, verweisen darauf, dass sie dabei explizit politische Ziele verfolgen. In den Arbeitsgemeinschaften haben sie einen Freiraum für dieses politische Engagement gefunden. Die Themenfelder sind dabei vielfältig. Während sich RONJA beispielsweise für klimapolitische Themen einsetzt, engagiert sich ESRA mit ihren Mitschüler*innen gegen Rassismus. 
Die Schüler*inneninitiativen und Arbeitsgemeinschaften werden vollständig selbstbestimmt von den Jugendlichen organisiert. Sie werden bei Bedarf von Lehrkräften unterstützt. Den interviewten Schüler*innen ist es aber wichtig zu betonen, dass es ihre Projekte sind. Sie holen sich Unterstützung von den Lehrkräften nach Bedarf, organisieren ihre Aktionen und ihre Initiativen aber selbstbestimmt. „Genau, und da haben wir auch keine, also, das entsteht wirklich nur aus Schülern. " (RONJA: 130-131) Die Gruppen treffen sich regelmäßig und sind jahrgangsübergreifend zusammengesetzt.

Angetrieben wird ihr Engagement von einem allgemeinpolitischen Verständnis, das über die bloße Interessenvertretung für ihre Mitschüler*innen oder bildungspolitische Themen beziehungsweise den sozialen Nahraum der Schule hinausgeht (u. a. ESRA: 136-138). Wenngleich ihnen der allgemeinpolitische Charakter ihrer Arbeit wichtig ist, betonen sie auch den Wunsch, unmittelbar ihre Mitschüler*innen zu erreichen. Hier sehen sie eine besonders hohe Wirkungskraft. Ihr Ziel ist es, ihre Mitschüler*innen für politische Themen zu begeistern und Vorurteilen etwas entgegenzusetzen (RONJA: 115-127).

Vor allem die antirassistischen Initiativen sind dabei oftmals aus der bundesweiten Initiative „Schule ohne Rassismus - Schule mit Courage“ entstanden. Dabei haben sich die Arbeitsgemeinschaften in den Jahren nach dem initiierenden Projekt ihre eigenen Schwerpunkte gesucht. RONJA leitet die Schülerinitiative an ihrer Schule, die im Zuge der Bewerbung um das Siegel gegründet wurde.

Dann haben wir eine Schülerinitiative gegründet und die leite ich seit dem letzten Jahr. Da machen wir Projekte, die die Demokratie fördern oder erhalten und andererseits versuchen wir Aufklärungsarbeit zu betreiben und das gegenseitige Verständnis von verschiedenen Kulturen und auch der Flüchtlingsdebatte näher zu bringen und da einen Austausch zu ermöglichen. (RONJA: 101-111)

Auch die Gruppe, in der sich JOHANNES engagiert, ist mit den von ihnen organisierten Aktionen Teil des Bündnisses geworden. Bemerkenswert ist, dass sich JOHANNES bewusst ist, dass die Gruppe für das Siegel „Schule ohne Rassismus - Schule mit Courage“ womöglich keine weiteren Projekte realisieren müsste. Er sagt dazu: „Wir müssen jedes Jahr eine Aktion machen, um das Siegel zu behalten, eigentlich. Das wird leider nicht wirklich durchgesetzt von der Stiftung, die das betreut. Denen ist das relativ egal. Wenn die das [Siegel] vergeben haben, dann bleibt das eben bei der Schule. " (JOHANNES: 507-510) Ob dieser Eindruck sich bestätigen lässt, soll an dieser Stelle nicht vertieft werden. Aus seinen Ausführungen wird aber deutlich, dass dieses Siegel für ihn und seine Mitstreiter*innen eher zweitrangig ist. 
Trotzdem haben wir uns vorgenommen, das einzuhalten, einmal im Jahr eine Aktion zu machen. Und das wird eben unsere Aktion. Immer wieder Konzerte. Nächstes Jahr ist ein Konzert geplant, eben Open Air. Wir werden wahrscheinlich wieder ähnliche oder gleiche Sponsoren haben. Ja, also da sind wir schon wieder an der Arbeit. (JOHANNES: 507-514)

Im Zuge ihrer politischen Arbeit in den schulischen Initiativen und Arbeitsgemeinschaften haben die interviewten Schüler*innen Aktionstage und Bildungsveranstaltungen wie Workshops, Podiumsdiskussionen und Vorträge organisiert. Sie haben Benefizkonzerte realisiert, Plakate und Flyer entworfen und politische Beiträge für die Schüler*innenzeitung geschrieben.

Das Handlungsfeld Schule haben die Jugendlichen für ihre politischen Aktionen bewusst ausgewählt. Ihnen ist es ein Anliegen, ihre Mitschüler*innen zu erreichen. Neben politischen Aktionen verstehen sie es auch als ihre Aufgabe neue Mitglieder zu gewinnen, um die Arbeit nachhaltig an der Schule zu etablieren. Dafür gehen die Schüler*innen regelmäßig in die Klassen und erklären ihre Arbeit.

Wir versuchen und strengen uns an und gehen manchmal sogar einmal wöchentlich durch die Klassen, stellen uns auf verschiedene Arten und Weisen vor und versuchen, es irgendwie für die Siebtklässler witzig und lustig darzustellen, damit es irgendwie interessanter ist. (ESRA: 155-159)

Vor allem die politische Aktion an der Schule ist für die Jugendlichen oft auch Empowermentarbeit. Es geht ihnen darum, über Themen wie Rassismus und Sexismus aufzuklären und ihre Mitschüler*innen zu sensibilisieren und zu bestärken (ESRA: 44-147). Außerdem wollen sie Anlässe schaffen, die es ihren Mitschüler*innen ermöglichen, einen Perspektivwechsel zu vollziehen. So hat RONJA mit ihrer Schüler*inneninitiative beispielsweise eine Geflüchtete aus Afghanistan eingeladen, die von ihren Erlebnissen auf der Flucht berichtet hat.

Sie ist auch Anfang zwanzig und sie hat schon einmal einen Vortrag hier in Anklam gehalten, über Frauenrechte in Afghanistan. Und sie wird den noch mal dann halten. Da werden wir es hoffentlich noch ein bisschen weiter vorbereiten, dass wir vorher noch mal das Land ein bisschen vorstellen. Afghanistan, was ist da los und danach noch mal so eine Diskussionsrunde oder Fragerunde. Da habe ich jetzt aber auch zum Beispiel im Vorfeld jetzt schon eine Frauengruppe angesprochen, ob sie sich da nicht mit reinsetzen können, weil das ja auch schon ein kritisches Thema ist. Wir sind jetzt nächste Woche auf Kursfahrt, das heißt, ich habe eigentlich auch keine Zeit mich damit auseinanderzusetzen und einfach, dass sie noch mal mit dabei sind, um vielleicht irgendwelche Fragen aufzufangen. (RONJA: 472-483) 
Dieser Erfahrungsbericht von RONJA steht beispielhaft für das reflektierte Vorgehen der junge Schüler*innen. Immer wieder wird deutlich, dass sie mit ihren Aktionen Prinzipien der politischen Bildung unbewusst anwenden. Sie wollen begeistern, aber nicht überwältigen, wollen möglichst viele Perspektiven aufzeigen und ihre Mitschüler*innen befähigen, kritisch und reflektiert ihre Interessen, aber auch die Interessen anderer zu erkennen, wahrzunehmen und sich im Idealfall mit ihnen gemeinsam zu engagieren.

Wie wichtig die Bedeutung dieser Bildungserfahrungen für die Jugendlichen sind, wird an folgendem Bericht von JOHANNES deutlich:

\begin{abstract}
Also, vor allem, was mir wichtiger war als das Konzert an sich, waren die Workshops, die damit einhergingen. Es wurden dann wirklich viele Workshops gegeben, auch zum Thema Rassismus und die waren alle in irgendeiner Form gegen Rassismus, aber eigentlich mit immer anderen Sachen verknüpft. Und ich finde solche Workshops immer wichtig, weil es bringt nichts, mir etwas anzuhören, ich muss selber etwas machen. Das ist bei mir jedenfalls immer so. Und ich denke, dass es bei vielen andern auch so ist. (JOHANNES: 344-350)
\end{abstract}

JOHANNES und seine Mitschüler*innen haben einen Aktionstag mit abschließendem Benefizkonzert organisiert. Vor allem das Konzert hat Aufmerksamkeit generiert und die Gruppe hat dafür große Anerkennung erfahren. Es ist umso bemerkenswerter, dass JOHANNES die Workshops in den Vordergrund stellt, die im Laufe des Tages an der Schule stattgefunden haben.

\title{
Außerschulische autonome Jugendgruppen
}

Wie RONJA haben sich mehrere Schüler*innen, die zunächst in der SV aktiv waren und später dann in einer Arbeitsgemeinschaft, letztlich mit ihrer politischen Arbeit doch immer weiter aus der Schule zurückgezogen. Von der Schulleitung und den Lehrkräften hat sich RONJA mit ihrem politischen Engagement nicht ernst genommen gefühlt. Wie mehrere andere Schüler*innen berichtet auch sie, dass ihr vorgeworfen wird, sie wolle nur den Unterricht boykottieren. Nicht zuletzt deswegen organisiert sie sich mit ihren Mitstreiter*innen mittlerweile auch in einer außerschulischen Jugendbewegung (RONJA: 958-964). Andere engagieren sich außerschulisch auch in Streikkomitees sowie lokalen und regionalen Protestgruppen. Die Schule verliert dabei immer mehr den Zugriff auf diese Jugendlichen und ihr politisches Engagement.

$\mathrm{Zu}$ weiteren Aktionen der Jugendlichen gehören Bildungsveranstaltungen außerhalb der Schule. So berichtet unter anderem BEN (295-296 \& 309) von Lesekreisen und Workshops, KATJA (574-580) von öffentlichen Diskussionsveranstaltungen, Podiumsdiskussionen und Vorträgen, FRANZISKA (758-768) 
von Protest- und Bildungscamps und RONJA (1010-1021) von Themenabenden und Waldspaziergängen mit einem Förster. Für ihre selbstorganisierten politischen Aktionen, aber auch für die (Selbst)Bildungsangebote entwerfen die Jugendlichen Flyer und Infomaterial (u. a. JAN: 259-260 \& 319-321; LAURENZ: 252-263).

Dabei wird deutlich, dass sich die Aktionsformen in der außerschulischen politischen Aktion verändern. Immer häufiger (,relativ häufig“ - HELGE: 216-217) nutzen die Jugendlichen unkonventionelle Partizipationsformen. Sie organisieren Demonstrationen gegen Sexismus (ESRA: 471-473; BEN: 335-335) und betonen den Wert großer, bunter und vielfältiger Protestformen (BEN: 413-415). Sie setzen sich in ihrem lokalen Wirkungskreis beispielsweise gegen das Erstarken rechtsradikaler Gruppierungen in ihrer Stadt (LAURENZ: 262-267; JAN: 121-128) ein, engagieren sich aber auch für sozialpolitische Themen wie die Solidarität mit Hartz-IV-Empfänger*innen (FRANZISKA: 478-485) und vernetzen sich mit internationalen Gruppierungen, wie den Demonstrationen „Pulse of Europe“ (LEA: 699-706) oder der „Fridays for Future“-Bewegung (RONJA: 706-709). Sie führen Solidaritätsaktionen für Geflüchtete durch (KATJA: 370373 \& 566-572; FRANZISKA: 478-485; SOPHIE: 157-170 \& 189-196), machen mit großen Transparenten auf politische Themen im öffentlichen Raum aufmerksam (SOPHIE: 528-538) oder gehen gegen Mobilisierungsmaßnahmen rechtsradikaler und islamophober Bewegungen vor, indem sie ihre Sticker und Plakate entfernen (LEA: 757-781).

Der Grat zwischen legalen Partizipationsformen und Formen des zivilen Ungehorsams bis hin zu politischer Gewalt wird dabei sehr schmal, wobei es sich bei dem überwiegenden Teil der illegalen politischen Aktionen eher um Formen des zivilen Ungehorsams handelt. Sie führen unangemeldete Demonstrationen durch oder organisieren Schulstreiks gegen marode Schulgebäude, gegen Rassismus oder für das Klima (u. a. BEN: 429-430; LEA: 106-106, 403-403, 735-740; ESRA: 468-470 \& 505-508; JAN: 137-140, 237-251, 271-280, 283-307 \& 315-319; RONJA: 728-733, 945-958 \& 1049-1061; SOPHIE: 523-528 \& 550561). Außerdem organisieren die Jugendlichen Blockaden und Besetzungen, die sie dabei vor allem in Bezug auf die Zielsetzung der politischen Aktion von den anderen Aktionsformen unterscheiden. Aktionsformen der unkonventionellen, aber legalen politischen Partizipation dienen den Jugendlichen dazu für ihre politischen Ziele einzutreten. Protestformen des zivilen Ungehorsams (beispielsweise Blockaden und Besetzungen) verknüpfen sie eher mit dem Ziel, sich gegen etwas zu positionieren oder gar etwas zu verhindern (BEN: 397-399). Die Bandbreite der Themenfelder ist dabei groß: Sie stellen sich Aufmärschen von Rechtsextremen in den Weg (FRANZISKA: 553-561), versuchen mit der Besetzung von Hauseingängen Abschiebungen von Geflüchteten zu verhindern (KATJA: 
572-574; SOPHIE: 157-170; BEN: 224-227) und besetzen ohne Genehmigung öffentliche Plätze, um auf Themen wie den Klimaschutz aufmerksam zu machen (FRANZISKA: 723-725; LAURENZ: 786-830). Grundsätzlich ist den interviewten Schüler*innen wichtig, dass auch der illegale Protest ohne Gewalt auskommt. Sie reflektieren aber auch diese Aktionsform. Wie die Jugendlichen Wirkungskraft und Zweck sowie die Legitimation der unterschiedlichen Protestformen einschätzen und reflektieren, wird im späteren Verlauf der Arbeit genauer betrachtet (siehe Abschnitt 4.3.4.5 „Reflexion des eigenen Handelns“).

\subsubsection{Konfliktfähigkeit - „Wenn die Klügeren immer nachgeben, ..."}

Für die vorliegende Studie ist von besonderer Bedeutung, inwiefern sich aus den Berichten der politisch aktiven Kinder und Jugendlichen Lernerfahrungen im Feld der Konfliktfähigkeit rekonstruieren lassen, denn gerade hier wird ein großes Potenzial in der politischen Aktion vermutet. Reales politisches Handeln bleibt nie unberührt von Konflikten. Inwiefern die selbstbestimmten und selbstorganisierten Aktionsformen dafür ebenfalls einen Erfahrungsraum bieten oder ob die Berichte der Jugendlichen eher darauf schließen lassen, dass die politisch autonome Aktion sich als geschlossenes System erweist, das auf Meinungshomogenität setzt und damit Konflikte eher vermeidet, wird im Folgenden herausgearbeitet. Für die Rekonstruktion von politischen Lernerfahrungen im Feld der Konfliktfähigkeit werden folgende Merkmale, angelehnt an die Überlegungen von Sibylle Reinhard (2018, 22 f.), zugrunde gelegt:

- Umgang mit Andersdenkenden zivilisieren

- ziviler Umgang mit Kontroversen

- Verzicht auf Gewalt

In der formalen Bildung werden unter diesen Gesichtspunkten zumeist institutionalisierte beziehungsweise geregelte Konflikte wie Gesetzgebungsprozesse oder Tarifverhandlungen im Sinne der Sozialpartnerschaft thematisiert. Für die vorliegende Arbeit stehen aber nicht institutionalisierte, sondern selbstbestimmte, autonome politische Aktionen und damit auch eher Konflikte im Fokus, die weniger von etablierten Strukturen (Herdegen 2017, 132) geprägt sind. Den Umgang mit Andersdenken zivilisieren heißt für die interviewten Jugendlichen vor allem, zu trennen zwischen Uneinigkeit in politischen Fragen und zwischenmenschlicher Beziehung. Das Politische nimmt für die jungen Menschen mittlerweile 
einen wesentlichen Teil ihres Lebens ein und prägt auch den Alltag, die Familien und den Freundeskreis. Umso wichtiger ist ihnen, dass das Politische nicht auch etwas Persönliches werden muss. Das lässt sich eindrücklich am Fallbeispiel ESRA nachzeichnen. Die junge Schülerin erklärt, dass sie auch mit ihrer Freundin, die sich ebenfalls politisch engagiert, oft uneinig ist. Sie diskutieren, sie sind verärgert, aber sie haben für sich erkannt, dass politisch unterschiedlicher Meinung zu sein, nicht bedeuten muss, auch persönlich in einen Konflikt zu geraten.

Zum Beispiel meine Freundin, die auch dabei ist, hat auch oft eine andere Meinung. Und danach sind wir manchmal sogar sauer auf uns gegenseitig, wenn wir diskutieren. Aber wir streiten uns niemals und kriegen uns immer wieder ein und sagen uns, jeder hat seine eigene Meinung und jeder darf es machen, darf es sagen, wie er will, und jeder kann sich auch äußern, wie er will. Und deswegen kriegen wir uns eigentlich immer wieder ein. (ESRA: 264-269)

In der Reflexion des Umgangs mit Konflikten ist es allen Jugendlichen besonders wichtig, und dabei ist kein Unterschied zwischen schulischen oder außerschulischen Wirkungsfeldern erkennbar, dass die Gruppenmitglieder ihr Verhalten reflektieren. Dabei wird die Diskussionskultur innerhalb der Gruppe immer wieder ausgewertet.

[Es gab dann eine Diskussionskultur,] mit der wir nicht mehr zufrieden waren, weil sie teilweise persönlich geführt wurde, also wo dann teilweise manche persönlich angegriffen wurden, was irgendwie überhaupt nicht geht. Das bedeutet also, wir müssen Inhalte kritisieren und nicht die Personen, die diese Inhalte äußern. Das ist etwas, eben ein Problem, das wir, würde ich sagen, erkannt haben und wo wir dann recht gut gegen vorgegangen sind, finde ich wenigstens. (BEN: 518-523)

Die Inhalte in den Fokus eines politischen Diskurses zu stellen und nicht die Personen, das ist eine wesentliche Erkenntnis, die die Jugendlichen auch durch ihr selbstbestimmtes politisches Engagement erlangten. Sie betonen aber nicht nur die Notwendigkeit, Person und Inhalt möglichst getrennt voneinander zu betrachten, sie weisen vor allem darauf hin, dass sie für sich den Wert von Konflikten als Wesenskern einer sich entwickelnden Gesellschaft erkennen. LAURENZ erklärt diesen Wert als Grundlage, um politische Diskurse zu entwickeln, Meinungen und Positionen zu bilden sowie Ideen und Lösungswege zu erkennen. Er ist gegen militärische Konflikte, findet aber den Konflikt wichtig im Sinne des kontroversen Ideenaustausches für die Bildung eines politischen Urteils und damit als Grundlage für politisches Handeln. 
Nein, ich möchte keine Welt, die frei ist von Konflikten, (..) Ich möchte aber dennoch eine Welt, die frei ist, von militärischen Konflikten zum Beispiel. Von gravierenden zwischenmenschlichen Konflikten. Ich möchte mich nicht unbedingt mit irgendwem wirklich streiten, ich möchte mich nicht mit jemandem prügeln. Aus was für Gründen auch immer. Ich denke aber, dass Konflikte nicht unbedingt sehr negativ sein müssen. Also, sie sind natürlich negativ konnotiert, das ist klar. Aber wenn ich mit jemandem eine politische Diskussion führe, ist das ja auch auf die ein oder andere Weise ein Konflikt. Und das finde ich sehr wichtig, weil wenn ich niemals mit jemandem diskutiert hätte, der nicht meine politische Meinung teilt, dann hätte sich auch meine Meinung überhaupt nicht entwickelt. Oder nur in nicht zufriedenstellendem Maße, aus meiner jetzigen Sicht. Also, Konflikte sind einerseits wichtig, aber andererseits kommt es eben auf den Konflikt an, welcher Konflikt das irgendwie ist. (LAURENZ: 512-525)

Den Ausführungen von LAURENZ ist zu entnehmen, dass er den Konflikt als wertvoll und dringend erforderlich einschätzt, weil er zivilisiert umgesetzt politische Willensbildung nachhaltig prägt. LAURENZ ist es dabei wichtig, dass Konflikte nur dann als Lernanlässe für die politische Urteils- und Willensbildung erfolgreich sind, wenn sie gewaltfrei geführt werden. Er betont, dass damit nicht nur körperliche Gewalt gemeint sei. Politische Konflikte sollten nach Möglichkeit frei von jeder Form von Gewalt sein, sei es „verbale Gewalt, physische Gewalt, psychische [oder] strukturelle Gewalt" (LAURENZ: 532-533).

Ein herausragendes Merkmal gelingender Entwicklung von Konfliktfähigkeit ist es, Meinungspluralität und Heterogenität im politischen Diskurs als wertvoll zu betrachten. Entgegen der Annahme, dass sich politische Arbeitsgemeinschaften oder autonome Jugendbewegungen eher homogen zusammensetzen, lassen die Erfahrungsberichte der Jugendlichen eher darauf schließen, dass sich die politischen Gruppierungen auch teilweise sehr heterogen zusammensetzen. Die selbstorganisierten politischen Arbeitsgemeinschaften an Schulen sind dabei besonders von Vielfalt geprägt, sowohl bezüglich der vorherrschenden politischen Positionen als auch bezüglich der Bereitschaft, sich an verschiedenen Formen politischer Partizipation zu beteiligen. Besonders hervorzuheben ist, dass die Jugendlichen diese Tatsache nicht als Hemmnis, sondern als befruchtend für die politische Arbeit begreifen. Besonders relevant erscheint in diesem Kontext der Erfahrungsbericht von KATJA. Sie ist vor ihrem Engagement in der schulischen Arbeitsgemeinschaft schon in einer autonomen politischen Jugendgruppe aktiv gewesen. Sie hatte vor allem Zweifel, weil sie eine zu große Heterogenität befürchtet hat, die dazu führen könnte, dass die Gruppe weniger wirkungsmächtig ist.

Es gibt halt total krasse Differenzen in dieser Gruppe, so was die politische Richtung angeht und auch die Weise, wie man die umsetzt oder wie man die umsetzen will. Und 
das läuft irgendwie trotzdem und das ist echt schön. Und die Gruppe ist total cool geworden. Und ich werde da bestimmt weiter machen, auch wenn ich jetzt nicht mehr auf der Schule bin. (KATJA: 220-225)

KATJA beschreibt den Wert schulischer politischer Aktion aus dem Vergleich ihrer Erfahrungen, die sie in einer selbstverwalteten Arbeitsgemeinschaft an der Schule und der autonomen Jugendbewegung gesammelt hat. Die Heterogenität der schulischen Arbeitsgemeinschaft, der sie anfangs skeptisch gegenüberstand, schätzt sie in der Rückschau als eine der größten Stärken ein (KATJA: 396-408).

Auch ESRA, die ebenfalls in einer schulischen Arbeitsgemeinschaft aktiv ist, hat vergleichbare Erfahrungen gemacht. Sie verweist darauf, dass die Gruppe sich in Bezug auf die Ziele der politischen Arbeit einig sei, die Wahl der dafür notwendigen Mittel aber immer wieder umstritten sei.

Ich denke, dass sind eher so Luxusprobleme, würde ich sagen. Klar, es entstehen mal Konflikte, weil vielleicht nicht alle derselben Meinung sind. Es gibt in der AG Leute, die sind schon fast, das darf ich jetzt, also ich weiß es natürlich nicht, ich denke schon fast linksextrem, was vielleicht viele aus unserer Gruppe gar nicht so toll finden. Ich bin es zum Beispiel nicht, aber ich bin auch offen dafür. Da gibt es halt manchmal Diskussionen, dass manche sagen: Ja, wir müssen jetzt die Schulwand ansprayen und irgendwie allen Leuten sagen, wir sind hier anti. Ja, da gibt es dann manchmal Diskussionen. (ESRA: 242-249)

ESRA betont allerdings den Wert pluraler Meinungen in ihrer Gruppe und die damit verbundenen offenen Diskussionen. Für sie stellt das keinen persönlichen Streit, sondern einen konstruktiven Meinungsaustausch dar. „Aber die Diskussionen sind keine Streitigkeiten, die Diskussionen sind Diskussionen, wo Leute ihre eigene Meinung sagen können. Und wenn jemand die eine oder andere Meinung hat, dann ist das so. Wir hassen uns auch nicht dafür." (ESRA: 257-264) Das trotz durchaus heterogener Zusammensetzung gemeinsam Projekte realisiert werden können, ist insbesondere der Tatsache geschuldet, dass die Schüler*innen in den Arbeitsgemeinschaften und politischen Gruppen lernen, mit realen Konflikten umzugehen. Daher hebt auch KATJA die Meinungspluralität in ihrer Gruppe als positiv hervor und betont, dass es gelungen sei, sich auf das gemeinsame Projekt zu konzentriere und die Gemeinsamkeiten in den Mittelpunkt zu stellen und nicht die Differenzen.

Da ist, glaube ich, allen relativ bewusst, dass wir verschiedene Meinungen haben und deswegen wird gerade nicht versucht, seinen Standpunkt durchzusetzen, sondern gesagt: Okay, wir versuchen, dass das Projekt an sich läuft und nicht irgendwie jetzt uns da an die Gurgel zu gehen, wegen irgendwelchen Kleinigkeiten und irgendwem 
unsere Meinung aufzudrücken. Das war auch irgendwie was, was ich gut fand an der Gruppe, auch zum Teil anders erwartet hätte vorher, glaube ich. (KATJA: 653-660)

Aber auch die Jugendlichen, die vorwiegend außerschulisch in autonomen Gruppen politisch aktiv sind, beschreiben Konfliktsituationen und verweisen ebenfalls auf den insgesamt heterogenen Charakter ihrer Gruppen. Auch in diesen Gruppen werden politische Debatten geführt und kontroverse Positionen abgewogen (FRANZISKA: 585-596). Die Jugendlichen schätzen aber nicht nur den Wert der Heterogenität innerhalb ihrer Gruppe. Sie wählen ihre Aktionsformen auch danach aus, inwiefern dadurch ein konstruktiv konflikthafter Austausch mit anderen Menschen erreicht werden kann. HELGE ist überzeugt, dass sich dafür beispielsweise Demonstrationen eignen (HELGE: 237-238).

Inwiefern sich Kompetenzen im Feld der Konfliktfähigkeit im Zuge des politischen Engagements der Jugendlichen entwickeln, soll im weiteren Verlauf dieses Kapitels anhand konkreter Beispiele nachgezeichnet werden. Für mehrere Schüler*innen-Gruppen war bereits in der Gründungsphase die Entscheidung über den Gruppennamen ein konflikthafter Prozess. Soll die Arbeitsgemeinschaft den Titel „gegen rechts“ oder „gegen Rassismus“ führen? Schon diese Frage führte zu politisch anregenden Diskussionen, offenbarte die Bandbreite vertretener politischer Positionen und konnte doch letztlich gruppenintern gelöst werden.

Es gab irgendwie die Überlegung, ob wir unser Projekt, gegen rechts' oder, gegen Rassismus ' nennen, weil die einen eben fanden, dass dieses, gegen rechts ' zu krass ist und wir ja eigentlich nur gegen Ausgrenzung und Rassismus sind und ,gegen rechts ', da steckt ja viel mehr dahinter und das klingt dann gleich so radikal. Und solche Diskussionen haben wir auch geführt. Und trotzdem funktioniert das irgendwie und wir kommen gut miteinander klar. Und das ist halt ganz spannend. (KATJA: 274-280)

Interessanterweise beschreibt KATJA, dass dieser Konflikt, trotz der damit verbundenen Brisanz, innerhalb der Arbeitsgemeinschaft schnell befriedet werden konnte. Später im Verlauf versuchte auch die Schulleitung zu intervenieren. Der Konflikt fand somit auf verschiedenen Ebenen statt und forderte den Schüler*innen auch eine Positionierung gegenüber der Schulleitung ab. Dabei ging es nicht nur um eine Beschreibung der Gruppe; der Konflikt um den Namen ist ein hochpolitischer Konflikt. Einigen Schüler*innen war die klare Positionierung „gegen rechts“ besonders wichtig, weil sie damit mehr als nur den Kampf gegen Rassismus verbinden. Für sie ist es auch ein klares Bekenntnis in Bezug auf die Frage, woher die größte Gefahr für unsere Demokratie und die freiheitlich demokratische Gesellschaft aktuell ausgeht. Wenngleich die Gruppe den Versuch der Einflussnahme durch die Schulleitung zunächst abwehren (oder eher aussitzen) 
konnte (KATJA: 288-398), haben sie sich letztlich ohne tiefergehende Auseinandersetzung mit dem Extremismuskonzept dem Wunsch der Schulleitung gebeugt und die Arbeitsgemeinschaft umbenannt.

Wir haben es einfach weiterlaufen lassen und sie hat sich nicht beschwert. Wir haben dann die AG, Schule ohne Rassismus ' genannt, aber haben weiter die T-Shirts [mit der Aufschrift, gegen rechts'] verteilt. Wir wollen unser nächstes Projekt jetzt allerdings ,für Toleranz' nennen, das Konzert war, gegen rechts ' und jetzt machen wir was ,für Toleranz: (KATJA: 324-328)

Aus politikdidaktischer Perspektive zeigt dieses Fallbeispiel, dass das reale politische Handeln offenbar vom ersten Moment an vielfältige Lernanlässe bietet. Hier wurde die Chance auf eine Auseinandersetzung mit diesem wertvollen Lernanlass versäumt. Letztlich einigte sich die Gruppe, die nächsten Projekte unter das Motto „für Toleranz" zu stellen. Eine Aufarbeitung des Themas ,Extremismus' und der konzeptionellen Ideen, die sich hinter den Begriffen verbergen, fand nicht statt.

Nee, nicht so richtig eigentlich. Vielleicht haben verschiedene Leute Toleranz unterschiedlich definiert, es hieß so. Naja, man kann ja auch Rechte bis zu einem gewissen Grad tolerieren, wo andere meinten, dass das nicht ginge. Aber so eine richtige Diskussion gab es da nicht noch einmal, aber vielleicht auch, weil die Leute, die gesagt haben, wir wollen nicht, dass das, gegen rechts ' heißt, schon so viel eingesteckt haben, weil es weiter so hieß, dass wir dann gesagt, okay, dann machen wir das jetzt , für Toleranz. und alles cool. Aber eigentlich waren damit alle halbwegs einverstanden. (KATJA: 333-340)

Wenngleich der überwiegende Teil der Schüler*innen den Wert der Meinungspluralität in ihren Gruppen hervorhebt, so zeigt dieses Beispiel eben auch, dass Kompromisse in den Gruppen nicht immer davon geprägt sind, dass die Argumente überzeugen, sondern dass der soziale Zusammenhalt nicht gefährdet werden soll.

Ein anderes Fallbeispiel zeigt aber auch, dass die Schüler*innen an politischen Positionen festhalten und eine fehlende Einigung auch das Ende der Arbeitsgemeinschaft bedeuten könnte. In der Vorbereitung eines Aktionstages gab es einen Streit darüber, ob eine Vertretung des Verfassungsschutzes für einen Workshop eingeladen werden solle. JOHANNES erklärt, warum er sich gegen diesen Vorschlag aussprach:

Weil der Verfassungsschutz gerade in den letzten Jahren bewiesen hat, dass er eben, gerade was rechten Terror angeht, nicht gerade die Kompetenz in Person ist und auch selber stark eben zum Erhalt dieser Machtstrukturen beiträgt, wie er schon sagt: 
Verfassungsschutz. Und auch viele Linke, also tatsächlich sogar prozentual mehr Linke als Rechte regelmäßig verhaftet und über die Buch führt und die beobachtet. Das waren dann eben nicht die Besten, um bei einem Konzert gegen Rassismus beziehungsweise den Workshops gegen Rassismus anzutreten. (JOHANNES: 365-372)

Der Konflikt um die Einladung einer Vertretung des Verfassungsschutzes führte dazu, dass mehrere Schüler*innen unzufrieden waren. Das Projekt hätte daran scheitern können.

Die Entstehung war ganz interessant, weil wir uns wirklich zusammengesetzt haben und dann eben dieses Problem mit dem Verfassungsschutz hatten, wo das wirklich kurz auf der Kippe stand, ob nicht die Hälfte einfach rausgeht, wenn der Verfassungsschutz kommt, aus dem Projekt, weil sie halt sagen: Das wollen sie nicht, mit dem wollen sie nichts zu tun haben. Der Verfassungsschutz wurde dann aber einfach gestrichen, das wurde dann nicht mehr angefragt. (JOHANNES: 379-385)

Für die Frage, inwiefern sich an diesem Beispiel Lernprozesse im Feld der Konfliktfähigkeit und Handlungskompetenz rekonstruieren lassen, ist bemerkenswert, dass dieser Konflikt nicht nur von Vorbehalten oder der Drohung, aus der Gruppe auszusteigen, geprägt war. Die Schüler*innen hatten ein großes Interesse daran, diesen Konflikt auch sachlich zu lösen. „Es gab einige, die dann Zeitungsartikel und generell Artikel rausgesucht haben und dann vorgestellt haben, warum sie das nicht wollen. " (JOHANNES: 393) Erstaunlicherweise wurden die Sorgen und der Unmut der Schüler*innen bezüglich der Einladung des Verfassungsschutzes vor allem von den begleitenden Lehrkräften ignoriert. Die Drohung, aus dem Projekt auszusteigen, führte dazu, dass das Thema nicht abschließend diskutiert wurde. Letztlich hat die Gruppe für sich entschieden, dass das vielfältige Programm für den Aktionstag auch ohne eine Beteiligung des Verfassungsschutzes auskommen kann (JOHANNES: 393-401). Hätte es sich bei diesem Projekt nicht um ein freiwilliges, selbstbestimmtes Projekt der Schüler*innen gehandelt, sondern hätte es ein unmittelbares Abhängigkeitsverhältnis zu den Lehrkräften gegeben, wäre dieser interne Konflikt sicher anders ausgegangen. In jedem Fall war die Abarbeitung an diesem Konflikt ein wertvoller Lernanlass für alle Beteiligten.

Aber auch an anderen Fallbeispielen lässt sich die Vielschichtigkeit politischer Lernerfahrungen im Kontext des realen politischen Handelns rekonstruieren. Ein vor allem in politischen Gruppierungen, die sich als links markieren, bedeutsames Thema ist der Konflikt im „Nahen Osten“. Die Frage der Positionierung zu diesem Konflikt ist für viele politische Gruppierungen wichtig und zugleich eine besondere Herausforderung. LAURENZ berichtet davon, dass seine Jugendgruppe sich daher dafür entschieden hat, dieses Thema und die damit verbundenen Konflikte 
besonders intensiv zu bearbeiten. Sie haben einen internen Workshop erarbeitet, um sich mit dem Nahostkonflikt zu befassen. Ziel war es, eine gemeinsame Position zu finden. Die Abstimmung im Plenum ging mit einem Verhältnis von etwa $55 \%$ zu $45 \%$ sehr knapp aus. Daher wurde der Beschluss vertagt. Bei derart kontroversen Themen ist es der Gruppe wichtig, dass Gruppenpositionen nicht mit einer knappen Mehrheitsentscheidung herbeigeführt werden (LAURENZ: 551570). Elementare politische Entscheidungen werden innerhalb der Gruppen nicht mit einer einfachen Mehrheitsentscheidung abgeschlossen. Diese und weitere Lösungsstrategien legen nahe, dass im Zuge des Engagements in den politischen Gruppen Handlungskompetenz und Konfliktfähigkeit gestärkt werden.

Neben der Frage, welche Methode zur Entscheidungsfindung herangezogen wird, lassen sich weitere Strategien der Konfliktlösung aus den Erfahrungsberichten der Jugendlichen herausarbeiten. Um Konflikte über grundsätzliche Fragen zu vermeiden, verständigen sich mehrere Gruppen der interviewten Jugendlichen auf ein politisches Selbstverständnis. Das zeichnet sich in der Regel dadurch aus, dass „Dinge wie Sexismus, Homophobie, Rassismus [oder] Gewalt“ (FRANZISKA: 610) nicht akzeptiert werden. Für die Umsetzung konkreter politischer Projekte teilen sich die Aktivist*innen in Bezugsgruppen ein und entscheiden untereinander im Vorhinein über einen Aktionskonsens, um Konflikte bei der Durchführung der politischen Aktion zu verhindern (LAURENZ: 685-703).

Im Gegensatz zu offenen Fragen bezüglich der zu wählenden Aktionsform, die von Anfang an in Bezugsgruppen ausgelagert werden, ist es den Schüler*innen wichtig, inhaltliche Kontroversen zunächst im Plenum anzusprechen. Unabhängig davon, ob sich die Jugendlichen in der Schule oder in außerschulischen Kontexten treffen, sind die regelmäßigen Plenumssitzung allen Aktiven wichtig, um potenzielle Konflikte zu entschärfen. Diese Gruppentreffen sind immer öffentlich und frei zugänglich. Den Aktivist*innen ist das wichtig, und die damit verbundene Öffentlichkeit und öffentliche Auseinandersetzung mit politischen Konflikten empfinden sie bereits als einen politischen Akt (LAURENZ: 242-249).

Eine weitere Strategie zum konstruktiven Umgang mit Konflikten beschreibt BEN als das „, solidarische Kritisieren“ (BEN: 131-133; 456-463). Den Jugendlichen ist nicht nur das Verfolgen politischer Ziele wichtig, sondern auch der wertschätzende und respektvolle Umgang untereinander. Ein wesentliches Merkmal von Konfliktfähigkeit ist der möglichst zivile Umgang miteinander. Dessen scheinen sich alle Interviewpartner*innen bewusst zu sein. Sie legen teilweise so großen Wert darauf, dass sie Strategien wie das solidarische Kritisieren in gruppeninternen Workshops erlernen: 
Wir hatten jetzt aber auch zum Beispiel einen Diskussionsworkshop, also so Debattenkultur zum Beispiel. Weil es manchmal so ein bisschen aus dem Ruder gelaufen ist, also auch mit Polemik zum Beispiel. Und was auch ein Problem ist, dass wir da auch ein bisschen gucken müssen, dass wir da eine gewisse, also keine Kontrolle rein bekommen, aber dass das halt ein bisschen organisierter ist und ein bisschen solidarischer, wenn wir miteinander reden. Und dass es nicht so ist, dass wir uns alle ankeifen, als wären wir alle fünf Jahre alt. (BEN: 507-514)

BEN ist überzeugt, dass dieser Workshop erfolgreich war, und er hat das „,Gefühl, seitdem ist es deutlich besser geworden “ (BEN: 532). Die Diskussionskultur und der Umgang mit Konflikten spielen für alle interviewten Personen eine zentrale Rolle. Dabei ist es nicht ausschlaggebend, ob sich die Jugendlichen in einer autonomen Jugendgruppe organisieren oder in einer Arbeitsgemeinschaft in der Schule. Das wird deutlich, wenn man die Aussagen von LEA analysiert. Sie ist deutlich jünger als BEN und vorwiegend in ihrer rassismuskritischen Arbeitsgemeinschaft in der Schule aktiv. Auch sie betont, dass sachliches Diskutieren sowie die Reflexion des eigenen Verhaltens zu den wertvollsten Lernerfahrungen ihres politischen Engagements zählten (LEA: 843-856).

Vor allem persönliche Konflikte zwischen Einzelpersonen werden eher losgelöst vom Plenum aufgearbeitet. „Also, meistens sind solche Diskussionen mehr so unter Einzelnen, also in Einzelsituationen, mit Einzelpersonen oder dann in kleineren Gruppen, jetzt nicht auf einem großen Treffen. " (SOPHIE: 344-347)

Die Sensibilität für den Umgang mit Konflikten geht soweit, dass sich vor allem bei emotionalen Konflikten auch Menschen dafür verantwortlich fühlen, Konflikte zu schlichten.

Wir haben es immer geschafft, das zu regeln. Und wir hatten auch wirklich immer Leute dabei, die sich ganz besonders für so etwas verantwortlich gefühlt haben. Dass sie dahingegangen sind, nochmal versucht haben, Gespräche zu suchen, gerade in Situationen, wo es persönlich irgendwie schwierig geworden ist. Also, großes Drama hatten wir irgendwie nie. Da bin ich auch stolz drauf. (FRANZISKA: 585-596)

Später im Interview beschreibt FRANZISKA detaillierter, wie kleine, vor allem persönliche Konflikte in der Gruppe gelöst werden. Der direkte und schnelle Austausch ist ihnen wichtig. Im Zweifel kommt eine unabhängige dritte Person hinzu und versucht zu vermitteln.

Bei kleinen Sachen reden wir natürlich immer wieder miteinander. Also, der dumme Spruch oder so, da wird das dann vielleicht einfach angesprochen und es gibt irgendwie ein Gespräch darüber, warum etwas nicht in Ordnung ist und dann verstehen die Leute das auch. Aber ansonsten ist es einfach unser Mittel der Wahl, dass wir alles einfach 
immer alles sofort ansprechen und dass man in der Gruppe immer Verständnis findet. Also, wenn man zu jemandem hinkommt, wenn man ein Problem nicht lösen kann, dann gibt es eben ein Gespräch zu dritt, wo noch jemand dazukommt und dann hat sich bisher immer eine Lösung gefunden. Das ist ganz gut. (FRANZISKA: 610-618)

Die Jugendlichen haben in den verschiedenen Gruppen vielfältige Konfliktlösungsstrategien für unterschiedliche Situationen entwickelt, erprobt und eingeführt. Vom Gruppenselbstverständnis und dem Konsensprinzip bei elementaren Fragen, über Mehrheitsentscheidungen, Verlagerung in Bezugs- oder Arbeitsgruppen bis hin zur Mediation durch unabhängige Dritte. Die verschiedenen Strategien für den jeweils angepassten Umgang mit Konflikten zeigen, dass die politischen Aktionen und das reale politische Handeln in Selbstbestimmung und Selbstorganisation vor allem im Feld der Konfliktfähigkeit viele Anlässe für Bildungserfahrungen schaffen. Besonders hervorzuheben ist allerdings die Tatsache, dass die Jugendlichen die verschiedenen Strategien, ihr eigenes Handeln und ihre individuelle Rolle in der Gruppe immer wieder reflektieren. Die Wirkung des politischen Engagements auf die Fähigkeit zum Umgang mit Konflikten sowie die Fähigkeit zur Selbstreflexion wird an der folgenden Aussage von LEA besonders deutlich:

Und so kann man einfach, wenn man lernt, das zu reflektieren, was andere sagen und was man selbst sagt, was man zum Beispiel in der AG oder in einer Diskussion oder so lernt, dann ist es auch im echten Leben leichter, so Sachen zu reflektieren. Und das kann nicht nur irgendwie in der Schule helfen. Sondern auch wenn zum Beispiel man sich mit Freunden streitet oder so etwas, ist es auch immer gut zu reflektieren. Und das lernt man halt durch sich engagieren und selbst was machen, was man dann hinterher reflektieren kann und überlegen kann, was könnte man besser machen. (LEA: 637-644)

Wenngleich die Schüler*innen verschiedene Strategien entwickeln, um wertschätzend und respektvoll, möglichst kompromiss-/konsensorientiert mit Konflikten umzugehen, so ist ihnen zugleich immer auch wichtig, eigene politische Positionen zu beziehen und dafür einzustehen. Auch für LEA ist es wichtig, dass sich Menschen politisch positionieren und den politischen Konflikt nicht scheuen. Sie beschreibt die Notwendigkeit, sich politisch einzubringen, an der kritischen Würdigung der Redewendung: Der Klügere gibt nach. Sie hat an diesen Spruch immer geglaubt, aber in politischen Fragen zweifelt sie mittlerweile an diesem Ansatz und hält in gar für gefährlich:

Ich glaube, dadurch, dass ich mich halt mehr mit Politik auseinandergesetzt habe, ist es dann gekommen, dass ich gedacht habe: Naja, wenn der Klügere immer nachgibt, dann ist es so, dass die Dummen immer entscheiden können. Und wenn die Dummen 
immer entscheiden, was dadurch kommt, wenn die Klügeren nie ihre Meinung sagen und nie da mitmachen, dann kommt es zu Sachen, die vielleicht einfach die Klügeren total doof finden. Und selbst wenn es mehr Klügere sind, als dumme Leute, aber die Klügeren immer nachgegeben haben, dann ist es irgendwie doof, weil sich dann die ganzen Klügeren, die vielleicht eigentlich mehr sind und eine andere Meinung haben, gar nicht eingebracht haben, weil sie gedacht haben: Ja, ich gebe jetzt mal nach, weil es ist sowieso egal. (LEA: 671-686)

FRANZISKAs Aussagen ist zu entnehmen, dass sie sich diese Meinungspluralität und den Austausch, den politischen Streit zwischen den vermeintlich Klügeren oder Dümmeren auch für die Schule wünschen würde. Streit und Konflikt sei anregend und befruchtend für den politischen Kurs, darin sind sich alle interviewten Jugendlichen einig, aber sie definieren auch Grenzen: Diskriminierung und Hetze dürften in der Schule keinesfalls Raum erhalten (u. a. FRANZISKA: 1052-1063).

Wenngleich bei nahezu allen interviewten Personen vergleichbare Bildungserfahrungen im Feld der Konfliktfähigkeit identifiziert werden konnten, so fällt ein Beispiel hier heraus. JAN, der sich sowohl in der Schule als auch außerschulisch politisch engagiert, hat in der Schule schnell gegen Widerstände ankämpfen müssen. Er erhielt wenig Unterstützung für seine Projekte, weder vonseiten der Lehrkräfte noch von seinen Mitschüler*innen. Das Verhalten seiner Mitschüler*innen, der Lehrkräfte, aber auch sein eigenes Verhalten, das erklärt er selbstkritisch, führte immer mehr zu einer Vereinsamung, zu einer Verinselung und später auch zu einer Radikalisierung in seinem politischen Engagement. Diesen Schluss lässt schon seine Beschreibung der Grenze seiner Diskursbereitschaft $\mathrm{zu}$ :

Natürlich hätten auch Gespräche mit Leuten, die zum Teil anderer Meinung sind, einen da vorangebracht, wenn man sich gegenseitig versteht, wobei man da natürlich schon wieder unterscheiden kann. Ich zumindest jetzt hätte meine Probleme, mich mit einem Typen von der Jungen Union zu unterhalten. Da gab es auch schon einmal Versuche, die sind ziemlich früh gescheitert, aber dass man so, ich würde sagen, so eine linke Grundrichtung, dass man prinzipiell Leute, die auch sehen, es gibt Probleme, aber die die vielleicht anders lösen würden, dass man da irgendwie gemeinsam nach Möglichkeiten sucht, das bringt einen auf jeden Fall auch weiter. (JAN: 488-497)

JAN hat kaum Interesse am Meinungsaustausch mit Andersdenkenden und ist auch überzeugt davon, dass er von den politischen Kontakten, die er bisher aufgebaut habe, kaum profitieren könne. „Dort habe ich gar nicht so viel gelernt, weil wir natürlich sehr, sehr ähnliche Standpunkte hatten alle. “ (JAN: 358-359) JAN 
scheint in der Zeit seines politischen Engagements kaum Konfliktfähigkeit entwickelt zu haben. Für ihn war der politische Kampf ein isolierter Kampf. Dialoge scheinen für ihn wenig sinnvoll.

\begin{abstract}
Ja, inwiefern sollte da Dialog jetzt noch groß nützlich sein, zumal ich eben nicht viele erfolgreiche Dialoge zu verbuchen habe für mich, die ich jetzt wirklich für sinnvoll erachte. Gerade jetzt eben mit der Erfahrung als Schülersprecher, wie wenig die das aus dem Ministerium und Schulamt interessiert. Natürlich ist das ein Vorurteil, aber ich gehe davon aus, dass das dann nicht mehr sinnvoll ist. Und wenn Leute, dann vor allem so Kritiker, der Meinung sind, man solle mit den Leuten reden, dann sollen die das doch machen, wenn sie glauben, dass sie mit ihrem Dialog Erfolg haben. Ja, dann sollen die das einfach machen. Das ist, glaube ich, so der Grund, warum der Dialog auch dann manchmal nicht stattgefunden hat. Ja. Gut. (JAN: 553-564)
\end{abstract}

Inwiefern positive oder eben auch negative Effekte von den Schüler*innen reflektiert werden, soll im folgenden Kapitel genauer beleuchtet werden. Dabei werden die Aussagen der Jugendlichen in Bezug auf die Reflexion des eigenen politischen Handelns in die Kategorien ,Wirkungskraft und Zweck“ sowie „Legitimation der politischen Aktion“" sortiert.

\title{
4.3.4.5 Reflexion des eigenen Handelns
}

\subsection{Wirkungskraft und Zweck}

Um zu identifizieren, inwiefern sich aus den Erfahrungsberichten der Schüler*innen politisches Lernen rekonstruieren lässt, ist es bedeutsam, neben den praktischen Erfahrungen der Interviewten auch die Fähigkeit zur Reflexion in die Analyse einzubeziehen. Im Folgenden werden daher die Aussagen der Jugendlichen sortiert und eingeordnet, die darauf schließen lassen, dass sie ihr politisches Handeln und die jeweiligen Aktionsformen entsprechend ihrer Wirkungskraft und ihrer Legimitation reflektieren.

An erster Stelle steht allerdings zunächst die Frage, welche Rolle die politische Aktion, das reale politische Handeln für die Akteur*innen tatsächlich einnimmt. SOPHIE reflektiert das politische Handeln als ein Handeln, das nicht anderen Menschen die richtige Lösung oder Haltung präsentieren sollte, sondern Anregungen zum selbstständigen Denken geben muss.

Ja, man muss immer, wo ich mir denke, dass das Problem ist, man kann keine Leute wirklich nachhaltig gewinnen oder motivieren, indem man irgendwie von oben herab agiert. Man muss immer mit den Menschen agieren und das ist so, dass man gucken muss, dass man die Leute anspricht, aber nicht ihnen versucht, die eigene Meinung aufzudrücken, eher anregt, so dass die dann beim Denken selber darauf kommen. 
Eigentlich ist die Lösung, sich da zu engagieren oder mitzumachen. (SOPHIE: 570575)

Für SOPHIE sind daher Ausdruckformen des politischen Protests immer daran gebunden, dass durch diese Aktionsformen Mitstreiter*innen gewonnen werden können. Für FRANZISKA und ihre politische Jugendgruppe gelten vergleichbare Prinzipien. Sie betonen aber noch: „Für uns ist es weniger relevant, ob die Aktion, die wir durchführen, legal ist oder illegal, sondern wir wägen vor allem ab, dass wir Aktionen machen wollen, bei denen tendenziell jeder mitmachen kann. " (FRANZISKA: 795-798) Die Aussage, die auf den ersten Blick radikal erscheint, wird im weiteren Verlauf relativiert:

\begin{abstract}
Wir wollen keine Aktionen durchführen, die Leute abschrecken, wo Leute sich denken: Was ist das? Wir wollen ja nicht randalieren, sondern protestieren. Deswegen richten wir unseren Aktionen offen aus, sympathisch und tolerant und integrativ, sodass sich jeder auch spontan anschließen könnte. Wir achten bei unseren Demonstrationen darauf, dass wir nicht als schwarz-vermummter Block auftreten, sondern als bunte sympathische ansprechende Schüler, die wir ja auch sind. Wir müssen uns ja nicht verstecken. Und ansonsten reden wir immer ganz konkret über die Sachen, die wir erwarten, und legen bei Bedarfeinfach neu fest, was wir machen wollen. (FRANZISKA: 798-806)
\end{abstract}

FRANZISKA scheint die Bedeutung des öffentlichen Auftretens ihrer Gruppe sehr bewusst zu sein. Sie reflektiert nicht nur die Aktionsformen, sondern auch den Habitus, der damit verbunden ist, und die Möglichkeit, mit ihrem Protest noch Menschen für ihre politischen Ziele zu aktivieren. Dennoch ist FRANZISKA überzeugt, dass die Wirkungskraft von selbstbestimmt politisch aktiven Menschen außerhalb institutionalisierter Partizipationsformen verhältnismäßig gering ist. Das führt sie darauf zurück, dass insgesamt zu wenig Menschen aktiv sind. Ihrer Gruppe gelingt es aber immer wieder, mehrere hundert Menschen zu aktivieren (FRANZISKA: 822-831). Dennoch ist ihnen bewusst, dass die großen, die globalen Ziele, eine Welt ohne Rassismus, geprägt von Gerechtigkeit und Solidarität, nicht mit ihren Aktionsformen erreicht werden kann (FRANZISKA: 833-837). Ziel ist es dennoch, sich diesem großen Ziel mit jeder Aktion etwas anzunähern. Daher sind die Jugendlichen insgesamt auch überzeugt, dass sich der Aufwand für das politische Engagement lohne. HELGE begründet das damit, dass er Menschen erreichen könne und diese sich mit politischen Themen dann auseinandersetzten. „Ja. Ich glaube auf jeden Fall, der Aufwand ist es wert. Weil ich eben Leute erreiche, die vielleicht im Nachhinein sogar glücklich darüber sind, 
dazu etwas erfahren zu haben oder sich damit auseinanderzusetzen. " (HELGE: 425-427)

Die Wahl der Aktionsformen ist bei allen Schüler*innen, die für die vorliegende Studie interviewt wurden, begründet und wird im Nachhinein auf ihre Wirkungskraft hin untersucht. Die Reflektion der verschiedenen Aktionsformen steht sowohl in den autonomen Jugendgruppen als auch in den politischen Arbeitsgemeinschaften an den Schulen immer im Mittelpunkt der Plenumssitzungen. Unmittelbar nach einer Demonstration oder einer anderen Aktionsform wird diese in der Gruppe besprochen und reflektiert.

Nach jedem stattgefundenen Event, nach jeder Demonstration, nach jeder größeren Sache wird das auch im nächsten Plenum diskutiert. Das ist dann der erste Punkt auf der Tagesordnung. Reflektion über die vergangene Aktion. Und das bedeutet, dass dann berichtet wird, was geschehen ist, und dann wird individuell noch einmal die Meinung dazu geäußert: Ist die Demonstration gelungen? Hat mir das persönlich gefallen? Ist es das, was ich erreichen wollte? Haben wir das erreicht, was ich, auch persönlich, erreichen möchte? Oder war es die Zielsetzung, die ich mir auch gesetzt hätte? Das wird im Plenum diskutiert und dann wird eben ein (..) jetzt nicht irgendwie festgelegtes Fazit dann geschlossen, also, es wird dann teilweise im Protokoll festgehalten. Ist eben gut gelaufen, die meisten sind zufrieden damit, so in der Art. (LAURENZ: 712-723)

Wenn nach einer politischen Aktion zum Vorgehen für die Zukunft Uneinigkeit besteht, wird das neu diskutiert. „Das ist ja ein Teil des demokratischen Entscheidungsprozesses, meiner Meinung nach, also, dass aus Fehlern Schlüsse gezogen werden. Wenn jetzt eine Mehrheit sagt: Diese Aktionsform lehne ich vollkommen ab. Da kann ich niemanden bei unterstützen. Dann fließt das in die nächste Aktion mit ein und dann fließt das in den nächsten Entscheidungsprozess mit ein. “ (LAURENZ: 726-730)

Neben den Reflexionen zu den Aktionsformen lassen sich vor allem noch Erkenntnisse zur Reflexion der Handlungsbedingungen aus den Erfahrungsberichten der Jugendlichen ableiten. Insbesondere die Schüler*innenvertretung und ihre politische Wirkungskraft beschäftigen alle interviewten Jugendlichen. Wenngleich die Schüler*innenvertretung auch rechtlich zumeist kaum politische Handlungsspielräume im Sinne eines allgemeinpolitischen Mandats hat, so ist sie doch für die Mehrzahl der befragten Schüler*innen Ausgangspunkt ihres politischen Engagements gewesen. Anders als man es vermuten könnte, waren aber nicht die positiven Erfahrungen im Kontext von Mitwirkungsprozessen institutionalisierter Partizipation entscheidend, sondern vielmehr die Frustrationserfahrungen, die mit diesem Amt verbunden werden. Die Schüler*innen berichten gehäuft davon, dass sie das Amt als unpolitisch, wirkungsschwach oder gar bedeutungslos empfunden 
haben. Sie haben eher Erfahrungen der Alibi-Teilhabe und der Instrumentalisierung gesammelt. RONJA bedauert die Tatsache, dass sie die SV-Arbeit mit diesen negativen Zuschreibungen verbindet. Für sie waren diese Erfahrung maßgeblich dafür, sich über das Amt hinaus andere Formen der politischen Einflussnahme, auch im Kontext der Schule, zu suchen. „Ich muss dazu auch sagen, dass das bei uns eine nicht so besondere Bedeutung hat. Es ist ein Job, der im Grunde belegt werden muss, was ich ziemlich schade finde." (RONJA: 206-208) Auch andere Schüler*innen berichten davon, dass die SV kein „Potenzial“ (JOHANNES: 314322) habe und eher als bürokratisch und wenig wirkungsmächtig empfunden wird.

Und dann bin ich in die Bezirksschülervertretung gegangen. Und da habe ich dann überhaupt nichts erreicht. Das war ein total bürokratisches Gremium von Leuten, die sich einmal in der Woche getroffen haben und über irgendwas geredet haben. Und wir haben ein Jahr lang eigentlich nichts gemacht, außer Papier hin und her zu schieben und Politiker einzuladen zu irgendwelchen Debatten in irgendwelchen Aulas und so. Das hat irgendwie überhaupt nichts gebracht. (FRANZISKA: 472-478)

Allerdings kann konstatiert werden, dass die Mitwirkung in der Schüler*innenvertretung für engagierte Kinder und Jugendliche offenbar ein erstes Erprobungsfeld für Teilhabeprozesse ist. Mit der Wahl zum Schulsprecher verbindet beispielsweise JAN auch den Start seines Engagements und seiner Bereitschaft, sich für die Interessen und Belange anderer einzusetzen:

Dann in der 11. Klasse, eigentlich war das eher so ein Spaßgedanke damals Schülersprecher zu werden, bin ich dann fast schon da reingerutscht. Ich hatte auch schon davor immer eine politische Meinung und habe dann eben angefangen mich mehr und mehr zu engagieren, weil ich das so gesehen habe, dass ich mit dem Schülersprecher jetzt die Aufgabe bekommen habe, mich da jetzt vielleicht für andere einzusetzen. (JAN: 115-120)

Grundsätzlich wird die SV eher als Alibi-Partizipation wahrgenommen, sie wird verstanden als verlängerter Arm der Klassenleitung oder gar der Schulleitung (SOPHIE: 444-457). Politische Arbeit wird kaum mit der SV verbunden und dennoch betonen die Befragten die Bedeutung einer schulischen Interessenvertretung.

Sie reflektieren auch den Stellenwert politischer Arbeit und politischer Aktionen im sozialen Nahraum Schule. HELGE verweist beispielsweise darauf, dass in der Schule möglichst viele unterschiedliche Menschen erreicht werden könnten, die sich zuvor noch gar nicht mit politischen Themen befasst haben. „Einfach 
weil es in der Schule so ist, dass man viel mehr Leute erreicht, die sich davor gar nicht damit auseinandergesetzt haben. “ (HELGE: 460-462) Und er ergänzt: „So regt man eben viel mehr Leute zum Nachdenken an als Leute, die es eh schon getan haben." (HELGE: 467-468) Die Schüler*innen reflektieren die Erfolge ihrer eigenen politischen Arbeit in der Schule aber insgesamt sehr rational. JOHANNES beschreibt beispielsweise eine Situation, die ihn fast an seiner politischen Arbeit zweifeln ließ, ihn aber letztlich doch noch mehr bestärkt hat. Die Arbeitsgemeinschaft hat anlässlich des geplanten Aktionstages gegen Rassismus Solidaritätsarmbänder verkauft. An einem Tag seien zwei junge Mittelstufenschüler gekommen, einer davon dunkelhäutig, und haben Armbänder gekauft. Was die engagierten Schüler*innen dann erlebten, hat sie nachhaltig bewegt:

[D]ann wollten beide eins [Armband mit der Aufschrift, Schule gegen rechts '] kaufen, der erste hat eins gekauft und dann wollte der andere Junge eins kaufen und der erste sagte: Nee, der Neger kriegt keins. Und ist weggegangen. So etwas gab es dann natürlich auch. Das war so ein bisschen ein Schockmoment. Als ich dann gefragt haben, was das denn gerade war, meinte der andere: Das macht der immer. Da muss ich mir keine Gedanken machen, da mache ich mir keinen Kopf drüber. Was natürlich A) irgendwie eine Form ist damit umzugehen, B) aber einfach zeigt, dass 'Schule ohne Rassismus' einfach falsch ist. (JOHANNES: 441-448)

Gleichzeitig haben diese Armbänder auch Diskussionen in den Klassen hervorgerufen und damit eine, so empfindet es JOHANNES, längst überfällige Debatten ausgelöst. Das habe dazu geführt, dass ,auch noch einmal viel hochgekommen ist in den Klassen" (JOHANNES: 451-452). Mit der Tatsache, dass Rassismus für eine gewisse Zeit die Diskussionen und Themen in den Klassen und Jahrgangsstufen prägte, habe die Arbeitsgemeinschaft ein zentrales Ziel erreicht. Dennoch beschreibt JOHANNES seine politische Arbeit bis zum Zeitpunkt des Interviews insgesamt als wirkungslos oder gar ,nicht existent“.

Das Traurige ist, dass ich sie als quasi nicht existent beschreiben würde. Die Lehrer haben sich gefreut, die Schüler haben sich gefreut, aber verändert hat sich dadurch überhaupt nichts. Auch durch unsere Aktion, die Workshops, ist nichts anders geworden. Das kann man natülich auch nicht erwarten, dass immer sofort alles anders wird. Aber es hat sich halt wirklich nichts verändert. Es ist immer noch so, mir wird immer noch erzählt: Leute, die sagen, da und da werden Leute wegen ihrer Hautfarbe oder sonst etwas gemobbt. Und es hat halt niemand darüber nachgedacht, warum sie auf dieses Konzert gehen, was sie auf diesem Konzert machen. (JOHANNES: 518-526)

Hoffnung hat JOHANNES allerdings schon, dass zumindest ein paar Schüler*innen durch den Projekttag ,,anpolitisiert“ wurden (JOHANNES: 528-534). 
Aufgrund der Sorge, dass institutionalisierte Beteiligungsformen wie die Schüler*innenvertretung und etablierte Aktionsformen wie Workshops und Projekttage, nur wenig erfolgversprechend sein könnten, gewinnen für die befragten Jugendlichen unkonventionellere Beteiligungsformate an Bedeutung. MICHAEL ist selbst Teil der SV, aber überzeugt, dass beim Thema Rassismuskritik die Arbeit der Schüler*innenvertretung wenig erreicht habe. Die Demonstration gegen Rassismus, die sie organisiert haben, habe dagegen viele inspiriert. „So, was wir jetzt für Vorträge gehalten haben, für Reden gehalten haben, hat sie nicht so angesprochen hier in der Schule. Aber das außerhalb der Schule, dass es da weiterging, das hat viele aufjeden Fall noch inspiriert und mehr angesprochen. " (MICHAEL: 382386) $\mathrm{Zu}$ den unkonventionellen Partizipationsformen, die an Bedeutung gewinnen, gehören neben den legalen wie Demonstrationen auch Formen des zivilen Ungehorsams wie Streiks und Blockaden. Beide Formate verbinden die Schüler*innen vor allem mit dem Ziel, sich Gehör zu verschaffen. „Die Demonstrationen und Streiks dienten ja vorwiegend dazu, sich irgendwie Gehör zu verschaffen, auf sich aufmerksam zumachen. " (JAN: 526-528) Die Schüler*innen wollen damit Öffentlichkeit für die Themen und Herausforderungen der Gegenwart schaffen. Das scheint ihnen besonders wichtig zu sein. ,Wenn ich mich aber auf die Straße stelle, merken auch andere Leute, dass es vielleicht Probleme in der Gesellschaft gibt und sich dafür Leute auf die Straße stellen. Und ich glaube, das kommt einfach bei viel mehr Leuten an, als wenn ich mich nur in dieser AG engagiere." (ESRA: 520524) Auch BEN ist davon überzeugt, dass man mit Demonstrationen Menschen motivieren könne, sich mit den Zielen der Bewegung auseinanderzusetzen. „Das hat mich immer sehr beeindruckt. Und ich denke, das ist ein sehr gutes Mittel, um auch irgendwie Leute darauf aufmerksam zu machen, dass auch sie aktiv werden können. " (BEN: 428-432)

Und mittlerweile ist es so, dass ich das immer noch wichtig finde auf Demos zu gehen und solche Aktionen zu machen. Aber ich glaube mehr, dass es voll wichtig ist, dass es in so eine gesellschaftliche Diskussion rückt, die Themen, die wir eben machen wollen. Und, dass das eher nicht passiert, wenn sich alle darüber aufregen, dass die Linken die Stadt auseinandergenommen haben. Auch wenn es überhaupt nicht so war, aber es die Medien dann so suggerieren. Das weiß man ja, dass das so passiert und deswegen glaube ich, ist das so, dass ich jetzt mehr auf so auf Information und Bildung und so setze. (KATJA: 591-599)

Wie KATJA in diesem Beispiel verweisen die Jugendlichen aber auch auf die von ihnen wahrgenommenen Grenzen der Wirkungskraft ihrer Aktionsformen. JAN sagt beispielsweise, dass Demonstrationen und Streiks nicht das richtige Mittel seien, um in den politischen Diskurs einzutreten. Sie seien daher auch nicht 
wirklich nachhaltig. „Sehr nachhaltig waren sie tatsächlich bisher nicht, weil es dazu dann nach so einer Demonstration in eine Dialogphase übergehen müsste. Das ist bisher nicht geschehen." (JAN: 526-530) Auch SOPHIE hält Demonstrationen grundsätzlich nicht für besonders wirkungsmächtig, weil sie überzeugt ist, dass die meisten Menschen vorbeigingen und sich dafür nicht interessierten. (SOPHIE: 518-523). Auch HELGE stützt diese These und ist überzeugt, dass Demonstrationen eher geeignet seien für jene, die sich schon für politische Themen interessierten. Sie seien eher ein Ausdrucksmittel politischer Positionen als ein Ort, sich mit Themen zu befassen. (HELGE: 468-475) Auch SOPHIE sorgt sich darum, dass man mit Demonstrationen nur die Menschen erreiche, die schon politisch seien. Außerdem kritisiert sie, dass diese Protestform für viele Menschen nicht zugänglich sei.

Und bei so Kundgebungen ist das manchmal auch einfach ein bisschen lächerlich (lacht), find ich. Deswegen weiß ich nicht, ob es immer die, also, es gab Momente, ich war schon auch auf echt coolen Kundgebungen dabei, aber, keine Ahnung, da war vor einem der letzte Abschiebeflieger, der nach Afghanistan ging, eine Kundgebung mit richtig guten Redebeiträgen. Aber die Leute, die da waren, das sind so immer dieselben Leute. Das ist traurig eigentlich, das sind die, die im Vorfeld, darauf angesprochen wurden und die wissen: Okay, meine Gruppe hat da einen Redebeitrag oder so etwas, oder ein Bekannter von mir. Dann gehen die da hin. Es kommen dann irgendwie so Leute, die dann so vorbeilaufen, die fühlen sich dann gar nicht angesprochen und trauen sich vielleicht nicht stehen zu bleiben oder so etwas. Ich weiß es nicht, weil das Ganze dann vielleicht auch so ein bisschen explosiv wirkte, mit den ganzen Transpis, die dann rumhängen und die meisten Leute in schwarz (lacht). Deswegen habe ich mir gedacht, auf der nächsten Kundgebung gibt es bunte T-Shirts, obwohl ich bunte T-Shirts auch hasse wie die Pest, aber mein Gott, man muss Kompromisse eingehen manchmal. Ich glaube, die besten Aktionsformen sind einfach direkt auf Leute zugehen und möglichst authentisch und man selbst zu sein und nicht, wie ich schon gesagt habe, eigentlich. (SOPHIE: 575-593)

Wie SOPHIE lehnt der überwiegende Teil der befragten Jugendlichen militante Demonstrationen beziehungsweise klassische "Schwarze-Block-Demonstrationen" ab. Sie halten diese Form des Protests für wenig zielführend. „Also nicht so die klassischen Schwarzer-Block-Demonstrationen, wo sich alle vermummen und mit schwarzer Sonnenbrille auf die Straße stellen. Ich denk, dass das eigentlich langfristig viel, viel mehr abschreckt, als dass es irgendwie auch versucht aufzurütteln. " (BEN: 409-413)

Auch bezüglich der Wirkungskraft illegaler Protestformen des zivilen Ungehorsams ist eine gewisse Ambivalenz in den Einschätzungen und Reflexionen 
erkennbar. ESRA erklärt die Wirkungskraft der Schulstreiks damit, dass Schüler*innen damit viel Aufmerksamkeit für das Thema in der Öffentlichkeit erreichten. Es regt zum Nachdenken an, und das ist für ESRA bereits ein großer Erfolg dieser Aktionsform (ESRA: 508-514). FRANZISKA hatte Formen des zivilen Ungehorsams wie Bildungsstreiks zu Beginn ihres Engagements nur wenig unmittelbare Wirkungskraft zugeschrieben, äußerte sich schließlich aber sehr beeindruckt vom Erfolg der Aktionen.

Von Leuten, die regelmäßig den Schulbetrieb und auch Unibetrieb bestreikt haben, ungefähr drei oder vier Mal im Jahr, nicht dahin gegangen sind und stattdessen riesige Demonstrationen gemacht haben, zentral und dezentral, vor dem Kulturministerium und so weiter. Und mich hat das total beeindruckt damals, dass wir damit Erfolge erzielt haben. Also, ich hätte das früher nie gedacht. Ich dachte immer, demonstrieren ist irgendwie so eine schöne symbolische Tätigkeit, aber das hat tatsächlich dazu geführt, dass hier bei uns, die Studiengebühren abgeschafft worden sind. Und die Kopfnoten waren dann auch nicht mehr auf meinem Zeugnis. Und das hat mich einfach total begeistert, dass man so einen Druck aufbauen kann, auf der Straße, von Schülern aus, die sagen: Nee, Leute ihr könnt das nicht einfach über unseren Kopf hinweg entscheiden. Wir sind damit nicht einverstanden. Wir wollen das anders haben. Und obwohl wir natürlich nicht alles erreicht haben, was wir wollten, hatten wir da eben diese Erfolge. Das hat mich total beeindruckt. (FRANZISKA: 458-472)

In Bezug auf Blockadeaktionen wird das Spannungsfeld aber noch deutlicher sichtbar. Einige Jugendlichen verweisen auf den unmittelbaren Erfolg solcher Aktionen und berichten über aus ihrer Sicht erfolgreiche Blockaden von Aufmärschen der Neonazi-Szene (BEN: 397; LAURENZ: 599) und verhinderten Abschiebungen (KATJA: 559; SOPHIE: 157; BEN: 224). Wenngleich eine verhinderte Abschiebung auch für KATJA prägend für ihr weiteres politisches Engagement war, hat sie die Sorge, dass direkte politische Aktionen im öffentlichen Meinungsbild in Verbindung gebracht werden mit Gewalttaten. Deshalb engagiert sie sich eher für Projekte im Kontext von Informationen und Bildung (KATJA: 591-599).

Anders als die meisten Befragten betont LAURENZ, dass illegale Formen des politischen Protests wie Blockaden immer auch verbunden sein müssten mit der Bereitschaft, passiv Gewalt anzuwenden. Die Konfrontation mit der Polizei oder den rechtsradikalen Demonstrant*innen sei dabei unabdingbarer Teil der Protestform (LAURENZ: 599-619). Er legitimiert in seiner Argumentation auch Gewalt gegen seine politischen Gegner*innen und meint damit neben den Rechtsradikalen auch Polizist*innen. Er begründet diese Bereitschaft zur Gewalt damit, dass die Gesellschaft von struktureller Gewalt geprägt sei und damit manchmal Gewalt das einzige probate Mittel sei. Die hier dokumentierten Aussagen, werden nur 
wenige Minuten nach einem klaren Bekenntnis zur Gewaltfreiheit in politischen Auseinandersetzungen (LAURENZ: 532-533) getroffen. Auch an dieser Stelle des Interviews relativiert LAURENZ diese Haltung wieder und verweist darauf, dass er sich klar von Terrororganisationen wie der Roten Armee Fraktion (RAF) abgrenze: „Das bedeutet nicht, dass man wie die RAF herumläuft und wahllos reiche Menschen erschießt oder Supermarktketten in die Luft jagt. Das ist überhaupt nicht das, was ich jetzt darunter verstehe. " (LAURENZ: 631-656) Dennoch: LAURENZ bewegt sich mit dieser Argumentation klar außerhalb dessen, was mit zivilem Ungehorsam (siehe Abschnitt 2.1.2.3 „Legal, illegal, ungehorsam“) verbunden wird und auch außerhalb des Aktionsfeldes, das pädagogisch begleitet werden könnte.

Die Aussagen von LAURENZ verdeutlichen, dass die Reflexion des eigenen politischen Handelns über die Dimensionen von Wirkungskraft und Zweck der Aktion hinausgehen. Es steht dabei immer auch die Frage der Legitimation ausgewählter Aktionsformen im Raum. Im folgenden Kapitel werden Aussagen und Reflexionen der interviewten Schüler*innen auf dieser Ebene rekonstruiert.

\subsection{Legitimation der politischen Aktion}

Nach den Aussagen von FRANZISKA gelten für die Auswahl ihrer politischen Aktionsformen bestimmte Grundsätze. Für sie ist nicht entscheidend, ob eine Aktion legal oder illegal ist. Für sie ist entscheidend, ob möglichst viele Menschen daran partizipieren könnten und wollen würden (FRANZISKA: 795-806).

Die Frage der Legitimation stellt sich vor allem in Bezug auf illegale politische Aktionsformen und dabei insbesondere Formen des zivilen Ungehorsams. BEN erklärt, warum er sich nicht an den G20-Protesten in Hamburg beteiligt hat. Er beschreibt die geplanten Aktionen nicht als illegitim, aber er könne sie nicht mit seinem Aktionsverständnis vereinbaren:

Dann da gibt's halt andere Aktionen, zum Beispiel wenn man gegen den G20-Gipfel demonstriert jetzt in Hamburg. Dass ich da nicht das Gefühl hätte, dass ich irgendwie mit denen irgendwie was zu tun haben wollen würde, weil die da irgendwie nicht in meinen Aktionskonsens reinpassen und in mein politisches Bild und ich das Gefühl hätte, dass meine politische Meinung dadurch verfälscht würde. Und das würde ich nicht wollen. (BEN: 420-426)

Blockaden von Veranstaltungen, wie sie bei den G20-Protesten durchgeführt wurden, die Verhinderung von geplanten Abschiebungen oder das sich Entgegenstellen bei Demonstrationen von Neonazis - all diese Fallbeispiele könnten 
als Aktionsformen des zivilen Ungehorsams verstanden werden. KATJA beschäftigte diese Protestform. Ihr ist bewusst, dass die Beteiligung an einer Blockade von Naziaufmärschen „,keine rechtliche Grundlage“ habe. Sie hält sie dennoch für legitim (KATJA: 562-565). Auch BEN hält diese Aktionsform für legitim, weil rechte und fremdenfeindliche Hetze für ihn keine Meinung, sondern ein Verbrechen seien.

Ich möchte jetzt keine Debatte über Meinungsfreiheit zum Beispiel aufmachen. Das ist ja sehr oft so, dass linken oder antifaschistischen Bewegungen vorgeworfen wird, dass sie die Meinungsfreiheit da nicht wahrnehmen. Also, keine Ahnung so, rechte und fremdenfeindliche Hetze ist für mich keine Meinung, sondern tendenziell ein Verbrechen. Und irgendwie denen, finde ich, sollte und dürfte man keine Bühne bieten. Und dementsprechend finde ich Blockaden ein mehr als zutreffendes und richtiges Mittel. (BEN: 401-408)

LAURENZ ist sich bewusst, dass dieses Vorgehen nicht ohne zumindest passive Anwendung von Gewalt unter anderem durch Widerstandshandlungen möglich ist. In einer Gesellschaft, die von struktureller Gewalt geprägt sei, davon ist LAURENZ überzeugt, sei dies aber manchmal ein legitimes Mittel (LAURENZ: 631-656). Er begründet die Legitimation des Einsatzes von Gewalt nicht nur mit dem zu erreichenden Ziel, der Verhinderung einer Raumgewinnung rassistischer oder faschistischer Ideologien in der öffentlichen Sphäre, sondern legitimiert Gewalt gegen Polizist*innen auch mit dem, in seinen Augen, ungerechtfertigten Einsatz von Gewalt durch Polizist*innen. Es zeichnet sich ein Feindbild ab. Gewalt gegen Polizeikräfte scheint für ihn damit grundsätzlich bei Blockadeprotesten legitim.

Aber wenn wir jetzt zum Beispiel einen vor Kurzem stattgefundenen Protest nehmen, wo die Demonstration vonseiten der Demonstranten weitgehend friedlich verlaufen ist, die Polizisten aber an erster Stelle mit Gewalt gekontert haben, schon als wir versucht haben die Demonstration anzuhalten, wurde direkt Pfefferspray in Massen eingesetzt, es wurden Schlagstöcke eingesetzt, die Polizisten sind auch mit Fausthieben und Tritten gegen die Demonstranten vorgegangen. Und ein Demonstrant wurde unter anderem auch von einem vorbeilaufenden Polizisten, der seinen Helm beim Laufen ausgezogen hat und ihm ins Gesicht gestoßen hat, recht schwer verletzt und hat geblutet. Und das ohne jetzt einen wirklich tätlichen Angriff auf irgendeinen Polizisten. Da steht die Gewaltfrage seitens der Polizisten. Die haben sie für sich auch schon geklärt an dieser Stelle. Ganz zu schweigen von den Nazis, die auch schon in kleinen Gruppen mit Messern angereist waren und die haben sich die [Gewaltfrage] auch nicht mehr gestellt. (LAURENZ: 657-669) 
Mehrere befragte Schüler*innen (u. a. auch JOHANNES: 172-179), deren Aussagen in den Interviews insgesamt zweifelsfrei darauf schließen lassen, dass sie sich mit politischen Zusammenhänge zuweilen rational und kritisch reflexiv auseinandersetzen, scheinen, auch aufgrund individueller Erfahrungen mit der Bereitschaftspolizei auf Demonstrationen, das Vertrauen in den Rechtsstaat und das staatliche Gewaltmonopol zu verlieren. Auch FRANZISKA berichtet von einer Situation, die sie seither an den funktionierenden Rechtsstaat zweifeln lässt. Der Erfahrungsbericht über ihre Festnahme im Zuge einer nicht-angemeldeten Demonstrationen wird im Abschnitt 4.3.5.1 „Frustration und Anerkennung“ genauer beschrieben (FRANZISKA: 849-863). In jedem Fall bieten die hier beispielhaft vorgestellten Argumentationsstrategien einen wertvollen Lernanlass für politische Bildungsprozesse. Vor allem die vielfältigen Frustrationserfahrungen der Jugendlichen im Kontext politischer Aktionen und politischer Selbstorganisationen stellen Lernanlässe und Gelegenheiten pädagogischer Arbeit dar, vor der sich die formale politische Bildung nicht verschließen sollte.

\subsubsection{Politisches Selbstkonzept}

\subsubsection{Frustration und Anerkennung}

Anerkennungs- und Frustrationserfahrungen prägen die Erfahrungswelt von politisch aktiven Jugendlichen. Erste Erkenntnisse zu Erfahrungen der Frustration und der Anerkennung, die in diesem Kapitel dargestellt werden, wurden teilweise bereits in einem Aufsatz veröffentlicht, der neben der vorliegenden Studie auch erste Teilergebnisse der Begleitstudie zum Projekt „Politische Partizipation als Ziel der Politischen Bildung" der Bundeszentrale für politische Bildung (BpB) von Alexander Wohnig in den Blick nimmt. Diese vergleichende Analyse verschiedener Erfahrungsberichte offenbarte bereits,

dass reales politisches Handeln von Kindern und Jugendlichen, das über das kommunikative politische Handeln aus dem Politikunterricht hinausgeht, ein hohes Frustrationspotenzial hat, zugleich aber auch positive Anerkennungs- und Selbstwirksamkeitserfahrungen ermöglicht. (Kenner/Wohnig 2020, 128)

Diese im Zuge der gemeinsamen Veröffentlichung mit Alexander Wohnig aufgestellte These wird im Folgenden ausführlich begründet. Die Aussagen der Jugendlichen wurden hierfür im ersten Schritt in die Hauptkategorien Frustration und Anerkennung und in einem weiteren Schritt in Unterkategorien kodiert. Diese lassen sich dabei noch einmal unterscheiden durch Bezugsfelder auf der 
Beziehungsebene und den Dimensionen der politischen Aktion. Die folgende Beschreibung dieser Ebenen basiert auf den Überlegungen aus der oben genannte Publikation (Kenner/Wohnig 2020).

In Bezug auf die persönlichen Beziehungsebenen lassen sich aus den Untersuchungsergebnissen vier elementare Bezugsfelder für die engagierten jungen Menschen herausarbeiten: Familie, Freundeskreis, Mitschüler*innen sowie Lehrkräfte. Die Schüler*innen, die sich im Laufe ihres Erfahrungslernens in der politischen Aktion in einem ständigen Selbstreflexionsprozess befinden, gleichen dabei Selbst- und Umwelterleben (Lange 2008a) fortwährend in der Interaktion mit diesen vier Gruppen aus.

Neben diesen personalen Wechselwirkungen beeinflussen weitere (de)motivationale Faktoren die Anerkennungs- bzw. Frustrationserfahrungen der jungen Menschen. Hierzu zählen Erfolg und Misserfolg bei der Planung und Umsetzung der Projekte, Merkmale in Bezug auf den örtlichen (lokal/global) und temporären (kurzfristig/langfristig) Charakter sowie die thematische Spezifizierung. Dabei lässt sich - von dem Datenmaterial ausgehend - allerdings nicht konstatieren, dass eine kleine und damit möglicherweise nicht erfolgreiche Demonstration zwangsläufig als Frustrationserlebnis wahrgenommen wird. Gesellschaftspolitische Schlüsselprobleme wie Rassismus und Klimawandel lassen sich nicht mit einzelnen politischen Aktionen lösen. Den Teilnehmenden sind die großen Dimensionen des Themas bewusst, und sie können für sich erkennen, dass eine kleine Demonstration nur marginalen Einfluss nimmt, aber dennoch lokal wirkungsmächtig sein kann.

Zunächst werden im Folgenden anhand ausgewählter Aussagen der befragten Jugendlichen Frustrationserfahrungen dargestellt und eingeordnet, im Anschluss werden exemplarisch Berichte der Aktivist*innen zu Anerkennungserfahrungen rekonstruiert.

Besonders bemerkenswert ist die Erkenntnis, dass Schüler*innen, die in der Schule Freiräume für politische Aktion erkämpft haben, mehrheitlich zunächst Frustrationserfahrungen mit ihren Mitschüler*innen in Verbindung bringen. Politisches Engagement führt im schulischen Kontext und den sozialen Interaktionen eher zu Ablehnungserfahrung.

Wir bekommen aber irgendwie nie Leute zusammen. Ich denke, das liegt daran, dass es vielleicht nicht cool ist, sich zu engagieren, dass es peinlich ist zu sagen: Ich bin hier dafür da, dass unsere Schule vielleicht ein bisschen besser läuft, und dass wir sozusagen in der Schule helfen, dass es alles besser wird vielleicht auch. Und für viele Leute ist es einfach nicht schön, dass man sich außerhalb der Schulzeit noch in die Schule setzt und sich da engagiert und irgendwas plant. Das finde ich eigentlich schade. (ESRA: 159-165) 
Dieses Gefühl, das ESRA hier beschreibt, ist kein Einzelfall. Auch LEA hat diese Erfahrung gemacht. Sie erklärt, dass es keinen guten Ruf habe, wenn man freiwillig für eine politische Arbeitsgemeinschaft länger in der Schule bleibe (LEA: 448-449). MICHAEL führt das darauf zurück, dass politische Arbeit für viele Schüler*innen $\mathrm{zu}$ anstrengend sei und verweist darauf, dass sie nicht wüssten, wie viel Spaß politische Arbeit machen könne.

Ich denke mal, der erste Punkt ist, weil sich Schüler damit beschäftigen müssen, weil sie aufstehen müssen und irgendwas tun müssen und es einfach einfacher ist, nichts zu tun. Und sie gar nicht sehen oder viele nicht wissen, dass es eigentlich Spaß macht, zusammenzuarbeiten mit der Schulleitung und einfach was zu verändern. Denke ich. (MICHAEL: 101-105)

Dabei nehmen die Schüler*innen eine unterschiedliche Wertschätzung von freiwilligem Engagement war. RONJA ist überzeugt, dass persönliches Engagement in Musik und Sport deutlich mehr Förderung erhalte als politische Aktionen. „Sport oder Musik wird viel mehr akzeptiert als politisches Engagement. “ (RONJA: 1006-1007)

Dies ist offenbar darauf zurückzuführen, dass diese Formen der Partizipation eher als soziales Engagement wahrgenommen werden und auf lokale und unmittelbare Verbesserung der Lebensverhältnisse abzielen. Politische Partizipation zeichnet sich dadurch aus, dass langfristigere Ziele verfolgt werden, die nicht nur unmittelbar und lokal ihre Wirkung entfalten sollen.

Partizipation im Sinne eines sozialen Engagements wird sowohl in der Schule als auch im außerschulischen Kontext hoch angesehen. Programme zur Förderung des sozialen Lernens haben Konjunktur. Institutionalisiert werden diese Programme in Sozialpraktika an den Schulen und Freiwilligendiensten, wie dem Freiwilligen Sozialen Jahr (FSJ) oder dem Freiwilligen Ökologischen Jahr (FÖJ). Kinder und Jugendliche, die sich im Altersheim für die Bewohner*innen engagieren und mit ihnen Spieleabende veranstalten, Schüler*innen, die Putzaktionen auf dem Schulhof organisieren, junge Menschen, die Patenschaften für Geflüchtete übernehmen - sie alle erfahren in der Regel viel Anerkennung für ihre Aktivitäten (Kenner 2019). Ohne dieses Engagement schmälern zu wollen, bleibt doch zu konstatieren, dass es für politische Bildungsprozesse von großer Bedeutung wäre, auch das Politische in dem sozialen Engagement herauszuarbeiten (Wohnig 2017). Dazu gehört, dass in der Schule und in non-formalen Bildungssettings Macht- und Herrschaftsverhältnisse thematisiert werden können und Fragen von Inklusion und Exklusion nicht nur aus der Subjektperspektive betrachtet werden müssen. Das bedeutet, dass nicht nur gefragt werden sollte, was jede*r Einzelne tun kann, 
sondern inwiefern politische und gesellschaftliche Strukturen verändert werden müssen, um das Zusammenleben weiterzuentwickeln. Für die politische Bildung darf nicht gelten, dass sie jungen Menschen beibringt, Aufgaben des Sozialstaates zu übernehmen. Sie muss vor allem junge Menschen bei dem komplexen Prozess der kritischen Selbst- und Weltaneignung unterstützen. Kurz gesagt: Junge Menschen können sich jederzeit sozial engagieren, sie müssen aber auch in der Lage sein, die Ursachen für soziale Ungleichheitsverhältnisse zu identifizieren und dazu befähigt werden, sich für gesellschaftspolitische Veränderungen einzusetzen (Kenner 2019).

Dass vor allem soziales Engagement von Kindern und Jugendlichen Anerkennung erfährt und politische Partizipation eher auf Ablehnung stößt, nehmen mehrere Interviewpartner*innen wahr. Das führt auch dazu, dass politisches Engagement von Schüler*innen eher als soziale Teilhabe verstanden wird, um ihr Wirken zu relativieren. LEA beispielsweise erklärt, dass sie die von ihr gewählten Aktionsformen für sehr wohl politisch einschätze, aber das Gefühl habe, dass ihre Arbeit in der Antirassismus-AG oder im Schulstreik nicht als Teil von Politik anerkannt würde.

Also, es ist klar, dass jetzt nicht irgendwie, was ich mache, nicht irgendwelche international wichtigen Entscheidungen sind. Und natürlich ist es klar, dass ich auf einer anderen Ebene Politik mache als jetzt Bundestagsabgeordnete. Aber ich finde trotzdem, dass ich, wenn ich zum Beispiel halt zur Anti-Ra-AG oder zu einem Schulstreik gehe, dass ich dann trotzdem mich mit Politik befasse und selbst auch Politik mache. Dass das nicht das Gleiche ist, ist klar. Aber, dass es dann trotzdem nicht, oder oft nicht, als Politik in Erinnerung gerufen wird, oder daran gedacht wird, wenn der Begriff Politik verwendet wird, das ist ein bisschen ärgerlich, finde ich. (LEA: 102-110)

Auch HELGE beschreibt in seinem Erfahrungsbericht, dass er den Eindruck habe, dass er in seinem politischen Handeln nicht ernst genommen werde. LEA und HELGE sind beides junge Schüler*innen der Sekundarstufe I. Sie kritisieren die Geringschätzung ihres Engagements aufgrund ihres Alters als Herabwürdigung und Abwertung und beschreiben damit das Erleben von Adultismus (Ritz 2013).

Ich glaube, die Schule sagt, dass es nicht funktionieren kann, wenn Schüler sich alleine engagieren. Oder die Schule sagt eben: ja, okay, das sind Schüler. Die sollten sich mal mehr auf Schule konzentrieren. Habe ich wirklich erlebt, teilweise. Mir wird auch des Öfteren vorgeworfen, also sarkastisch gesehen, ich würde die Arroganz besitzen, mich als Schüler schon mit so etwas auseinanderzusetzen und hätte diese Selbstsicherheit schon fast in einer Form von Arroganz in mir. Dass ich sage: Okay, ich beschäftige mich damit so, dass ich schon meine Meinung dazu sagen kann. Und es ist sehr schade, leider, weil ich eben gerne das hätte, dass man sagt: Ja, okay, auch wenn teilweise 
die Sicht von Schülern nicht so breit gefächert sein kann, wie die von Erwachsenen, weil natürlich die Erfahrung noch nicht so stark da ist, dass man aber trotzdem sagt, wenn jemand Lust hat, sich mit Themen auseinanderzusetzen und schon an dem Punkt ist, sich zu engagieren für das, was er denkt, dass man das eben unterstützt und nicht sagt: Ja, okay, der interessiert sich jetzt seit zwei Jahren für solche Themen und macht das auch gerne und viel in seiner Freizeit, soll er mal machen. Aber deswegen müssen wir uns jetzt nicht damit auseinandersetzen, sondern das muss dann schon von ihm kommen. Und wenn es von ihm kommt, dann kann er machen, was er versucht, und soll es halt selber auf die Beine stellen. Aber wir müssen es nicht unterstützen. [...] Aber wenn diese Unterstützung viel mehr da ist, dann hätte ja auch ein Schüler viel mehr die Möglichkeit, viel mehr Erfahrung zu sammeln, und merkt auch, dass Unterstützung da ist. (HELGE: 628-650)

Lehrkräfte würden sich hauptsächlich für ihren Unterricht und für den Lehrplan interessieren. Nach den Worten von RONJA unterrichten Lehrkräfte Theorien und Konzepte zu Parteien und achten nicht darauf, dass die Schüler*innen zur gleichen Zeit selbstständig Podiumsdiskussionen mit Politiker*innen organisieren. Sie wünscht sich daher, dass „Lehrer überhaupt mehr darauf eingehen würden “, was die politisch engagierten Schüler*innen machen.

Und es wäre cool, wenn im PB-Unterricht nicht nur stumpf, wie bei dem einen Lehrer oder eigentlich im Allgemeinen ist es im PB-Unterricht so angelegt, dass wir die Demokratie, die Definitionen auswendig lernen und die Definitionen von Parteien. Aber die Lehrer juckt es überhaupt nicht, dass wir Podiumsdiskussionen der verschiedenen Parteien organisieren. Die gehen da auch nicht hin. Das ist null miteinander verbunden. Es ist nur Theorie, Theorie, Theorie, null Praxis. Und die Praxis lerne ich so. Und das ist das, was zählt. (RONJA: 1183-1191)

Sehr differenziert unterscheiden mehrere Interviewpartner*innen zwischen den von Lehrkräften unterstützen Projekt und ihren eigenen Initiativen. Vor allem für die von ihnen selbstbestimmt initiierten Aktivitäten würden sie sich mehr Rückhalt wünschen. KATJA, eine Berufsschülerin, stellt fest, dass schulisches Engagement, zumindest dann, wenn es von Lehrkräften initiiert beziehungsweise begleitet wird, eher anerkannt wird als selbstbestimmte, selbstorganisierte und außerschulische politische Aktionen.

Das wird lieber gesehen, wenn Schüler sagen, wir engagieren uns für irgendwas und das durch Lehrkräfte begleitet wird, als wenn Leute quasi autonom sagen, dass sie irgendwie etwas auf die Beine stellen wollen, das ist schon ein Unterschied. (KATJA: 383-390) 
RONJA, aktiv in einer politischen Schüler*innengruppe, hätte sich mehr Unterstützung durch die Schulleitung gewünscht. Diese habe immer wieder zugesagt, dass sie Freiräume schaffen würde, diese Zusage aber nie eingehalten (RONJA: 411-412; 921-938 \& 964-999). Besonders hervorzuheben ist, dass der Schülerin auffällt, dass die Schule durchaus öffentlichkeitswirksam die Erfolge der politischen Arbeit der engagierten Schüler*innen herausstelle, diese aber selbst nicht fördere. „Zum Beispiel auch das Schild 'Schule ohne Rassismus, Schule mit Courage' hängt direkt am Eingang der Schule [...], aber trotzdem ist es nicht so, dass wir davon irgendwelche Vorteile haben, uns zu engagieren. “ (RONJA: 157-164) HELGE berichtet gar davon, dass die Schulleitung Projektideen bewusst verhindere, weil die Schule nicht wolle, dass politisch brisante Themen wie Homophobie mit dem Schulnamen in Verbindung gebracht würden (HELGE: 497-514). Die Schüler*innen, die an ihren Schulen politische Aktionen planen und Freiräume für das politische Handeln in der Schule etablieren, berichten einhellig von diesen Erfahrungen. Gleichzeitig gibt es aber immer wieder auch Lehrkräfte, die sie unterstützen. Sie resignieren daher nicht, sondern setzen sich weiterhin dafür ein, dass politische Themen und Aktionsformen in den Schulalltag integriert werden.

Die Mehrzahl der interviewten Schüler*innen engagiert sich nicht nur in autonomen politischen Gruppen innerhalb und außerhalb der Schule, sondern auch in den etablierten und institutionalisierten Strukturen der Schüler*innenmitwirkung. Doch vor allem diese Partizipationsräume beschreiben sie als Alibi-Teilhabe. Immer dann, wenn Aktionen der Schüler*innen über bloße Unterstützungsprojekte wie Sozialpatenschaften hinausgehen und etablierte Institutionen der Schüler*innenmitwirkung einen politischen Charakter erhielten, waren die Jugendlichen ihren Worten zufolge mit Ausschlusserfahrungen konfrontiert. Schulleitung und Lehrkräfte reagierten mit Instrumentalisierung, Verboten oder gar Repressionen. SOPHIE war nach ihrem Freiwilligen Sozialen Jahr (FSJ) an der Berufsschule Klassensprecherin. Sie wollte sich hier engagieren, empfand die Zeit in der Schüler*innenvertretung aber als sehr ernüchternd. Sie beschreibt das Gefühl, von ihren Lehrkräften ausgenutzt zu werden:

Ich war dann tatsächlich auch an der Berufsschule dann Schülersprecherin, was mich dann allerdings wieder angekotzt hat, weil ich mir dann wieder gedacht habe: Okay, ich weiß wieder, warum ich das die ganzen Jahre über nicht gemacht habe. Weil man dann nämlich die ganze Drecksarbeit für die Lehrer machen muss, die keine Lust haben, so einen blöden Weihnachtsbasar zu organisieren. Dann steht man da und deine Aufgabe als Schülersprecher ist jetzt, diesen Weihnachtsbasar zu organisieren. Und du denkst dir: Toll, danke, auf genau so etwas habe ich wahnsinnig Bock. Dann habe ich in dem Jahr natürlich dementsprechend nicht viel gemacht als Schülersprecherin, außer das, was ich tun musste, und war dann eben wieder ein bisschen genervt von diesem 
sozialen Engagement und habe deswegen, glaube ich, auch so eine Weile gebraucht, bis ich da überhaupt in diese Gruppe mit reingekommen bin. Also warum ich da trotzdem immer gewartet habe und gesagt habe, vielleicht nach den Ferien und so. (SOPHIE: 444-457)

Vor allem dann, wenn Schüler*innen die SV-Arbeit nicht nur als soziales Engagement für die Gruppe, den Klassenverband, Jahrgang oder die Schule verstehen, sondern auch politische Themen zum Kern ihrer Arbeit machen, stellen sie fest, dass die Schule dafür kaum (Frei)Räume zu bieten scheint. HELGE sagt, dass er mit seinem politischen Engagement vor allem in der Schule fast immer scheitere. Erfolgreich laufen Initiativen der SV nach seinen Erfahrungsberichten nur dann, wenn Eltern oder Lehrkräfte sie unterstützen.

Gescheitert bin ich des Öfteren und erreicht, was ich wollte, habe ich fast immer nur, wenn von Elternseiten oder teilweise von Lehrerseiten aus, wie zum Beispiel der Sozialpädagoge oder Ethikfachleiter haben da sehr oft geholfen, Unterstützung kommt. Wenn wir das als Schülersprecher allein versuchten durchzusetzen, hat es eigentlich fast nie funktioniert. (HELGE: 608-612)

In seiner Rolle als Schülersprecher hat auch JAN die Erfahrung gemacht, dass er wenig Gehör finde und nicht einmal die ihm zustehenden Rechte wahrnehmen könne. Er fühlte sich dabei auch von der Bildungspolitik unzureichend unterstützt.

Als Schülersprecher habe ich dann auch die Erfahrung gemacht, also (...3...), dass wenn man auf irgendwelche Rechte pocht, die einem eigentlich gegeben sind, dass das Schulamt, das Bildungsministerium, dass die das einfach überhaupt nicht interessiert, ob das jetzt eingehalten wird. Die interessiert das nicht, dass man jetzt seine Rechte auch irgendwie wahrnehmen kann und sich einbringen kann. Das ist denen scheißegal. Und insofern wird es einem schon erschwert. (JAN: 83-89)

Er beschreibt Konflikte mit seiner Schulleitung, die nach seiner Aussage sein Engagement als Schulsprecher vom ersten Tag an behindert hat. Er habe unter anderem einen Verweis erhalten (JAN: 155-192).

Sollten die Aussagen der Schüler*innen zutreffen, kann geschlussfolgert werden, dass die derart hervorgerufenen Frustrationserfahrungen darauf basieren, dass Teilhabe in den institutionalisierten Formen der Schüler*innenmitwirkung eher auf der Ebene der Nicht-Mitwirkung/Alibi-Teilhabe (siehe hierzu ausführlich Abschnitt 2.1.3.2 „Zwischen Fremd- und Selbstbestimmung“) verharren. Das Gefühl, als Schüler*in übergangen zu werden, zeichnet für die interviewten Jugendlichen aber nicht nur die Arbeit in der SV aus. Auch in den weitgehend selbstorganisierten Arbeitsgemeinschaften schildern die Schüler*innen, 
wenn auch deutlich seltener, vergleichbare Situationen. JOHANNES berichtete beispielsweise, dass ein Vorschlag der Schüler*innen durch die unterstützenden Lehrkräfte, teils ohne eine Diskussion zuzulassen, abgelehnt wurde.

Aber das ist dann bei den Lehrern auf relativ taube Ohren gestoßen, die meinten: Nein, wir machen das und das ist jetzt so, da wird nicht mehr drüber entschieden, da wird nicht mehr drüber gesprochen. (JOHANNES: 394-397)

Doch auch alltägliche Fälle erschütterten das politische Bewusstsein der engagierten Jugendlichen. So hat BEN davon berichtet, dass er wegen des Tragens eines T-Shirts mit dem Aufdruck „Refugees Welcome“ des Unterrichts verwiesen worden sei. Der Vorwurf der Lehrkraft, so fasst BEN die Situation zusammen, das T-Shirt symbolisiere einseitige linke Propaganda. Darüber hinaus habe der Lehrer ihn darauf hingewiesen, dass „Politik in der Schule, also, Schule sei ein politikfreier Ort, weil man da rangehen müsse nach dem Motto: Dann gibt es ja Spaltung unter den Schülern und das ist alles total schrecklich. " (BEN: 544-549) Auch HELGE hat vergleichbare Erfahrungen gemacht und kommt für sich zu der Erkenntnis, dass er seine politische Meinung in der Schule nicht öffentlich machen könne (HELGE: 115-117). Später im Interview wird HELGE deutlicher: Für ihn steht fest, dass ,es nicht, also vor allem in Bezug auf Schule, nicht immer gut ist, das zu sagen, was man wirklich denkt. Das ist zwar ein bisschen traurig, aber das habe ich wirklich gelernt. " (HELGE: 606-608)

Auch FRANZISKA beschreibt eine frustrierende Erfahrung mit ihrem Politiklehrer. Seinen Unterricht empfand sie als einseitig. Ihre Initiative, einen Vortrag in einer EU-Reihe zu Frontex zu halten, hat ihr der Lehrer verboten.

Aber es gab auch Lehrer, die fanden das vor allem nervig. Die fanden das total schwierig und nicht in Ordnung. Für die war ich die absolute Querulantin. Die hätten mich am liebsten nicht in ihrem Unterricht gehabt. Also, ich habe zum Beispiel bei diesem Politiklehrer, bei dem wir die europäische Einigung durchgenommen haben, dem habe ich halt gesagt, dass ich seinen Unterricht einseitig finde und der mir auch keinen Spaß macht und ich ungern komme. Das fand der schon mal total anmaßend, dass ich ihm das gesagt habe. Dann habe ich ihm gesagt, ja, also, ich würde gerne eine andere Perspektive reinbringen zum Thema Europa und ich würde gerne ein Referat halten in Ihrem Unterricht zum Thema Frontex. Und dann hat er das halt verboten und er hat gesagt: Nee, das gibt es nicht. Das ist ein Thema, das gehört hier nicht hin in meinen Unterricht. Das hat er so gesagt. Und das hat mich total wütend gemacht und ich bin dann in seinen Unterricht eigentlich nicht mehr gegangen, das letzte Quartal vorm Abitur, weil das hat mir nichts gebracht. (FRANZISKA: 954-968) 
Noch deutlicher werden die Beschreibungen von Frustrationserfahrung im Zusammenhang mit autonomen politischen Aktionsformen der Schüler*innen. FRANZISKA beschreibt die Widerstände, die sie vor allem bei Aktionen wie Vollversammlungen, Schulstreiks und Demonstrationen in und mit der Schule erlebt hat, sehr eindrücklich:

\begin{abstract}
Aber viel interessanter finde ich eigentlich die Repression, die ausgeht, zum Beispiel von der Schule, wenn man sich politisch engagiert. Also, wir haben ja oft zum Beispiel den Schulbetrieb bestreikt. Das kam in Schulen oft gar nicht gut an. Also, wir haben weniger Erfahrung damit gemacht, wo Schulleiter und auch Lehrer die Idee eigentlich gut fanden und sich sogar selbst beteiligt haben, aber an anderen Schulen war es dann oft so, dass einem gesagt wurde: Nein, mach erstmal dein Abitur, dann kannst du dich immer noch politisch engagieren. Oder: Warum muss das denn in der Schulzeit sein, es geht dir doch nur ums Schwänzen. Das ist doch nur ein Vorwand, hier politisch und so, so ein Quatsch. Es würde ja niemand zu eurer Demonstration kommen, wenn ihr es nicht in der Schulzeit machen würdet. Oder, dass wir rausgeflogen sind aus Schulen, an denen wir Werbung machen wollten für unsere Aktionen, dass wir an den Schulen, die wir selbst besucht haben, keine Vollversammlung durchführen durften. Ich habe mal mitbekommen, wie einem Mitschüler von mir vom Schulleiter das Megaphon aus der Hand geschlagen worden ist, als er eine Demonstration damit bewerben wollte. Repression ist nicht nur, meiner Meinung nach, das, was direkt von der Polizei oder von anderen staatlichen Behörden ausgeht, sondern, was auch ausgeht von Institutionen, wie eben der Schule oder am Arbeitsplatz. (FRANZISKA: 880-897)
\end{abstract}

Auch der Schulstreik, den RONJA im Zuge der „Fridays for Future“-Bewegung initiiert hat, hatte großen Widerstand der Lehrkräfte ausgelöst:

Ich würde gerne über das Bündnis noch was zu Fridays For Future sagen ganz kurz. Und zwar haben wir mit 80 Personen angefangen, die mitgestreikt haben. Da war der Aufschrei extrem. Wir haben super viel Gegenwind von Lehrern bekommen, die angefangen haben, ohne, dass sie die Bewegung kannten, was auch schon ziemlich krass ist, weil ich meine, die ist so medienpräsent-man kann sie quasi kaum verpassen - die haben total angefangen gegen uns zu hetzten. Wir schwänzen, wir haben kein Hintergrundwissen und haben sich mit allem, was sie konnten, dagegengestellt. Keiner von denen war schon einmal auf unseren Demos. Wir haben auch schon in den Ferien gestreikt. Wir haben viel faktenbasierte Redebeiträge zum Beispiel, wir machen viele andere Aktionen und da kommt zum Beispiel auch kein Engagement der Lehrer. Aber andererseits diskreditieren sie uns andauernd. (RONJA: 945-956)

Die politisch engagierten Schüler*innen sammeln aber nicht nur in der Schule Erfahrung mit fehlender Unterstützung oder gar Repressionen. Davon schildern die interviewten Jugendlichen vor allem im Kontext autonomer politischer Aktionen, wie Schulstreiks, Demonstrationen und Blockaden. FRANZISKA berichtet 
beispielsweise von staatlichen Repressionen wie Festnahmen auf Demonstrationen. Sie glaubt seither nicht mehr daran, dass es ein ernsthaftes Interesse von politischen Verantwortungsträgern am politischen Engagement der Bürger*innen gebe.

Ich weiß nicht, es gibt jetzt diese Kampagne für mehr Demokratie: „Setz dich ein!“ oder so. Aber sobald man sich einsetzt, kriegt man irgendwie den heftigsten Gegenwind auf der Straße entgegengeblasen von diesem Staat. Also, ich war vor Kurzem auf einer Demonstration und wurde in Gewahrsam genommen. Dann fragt man sich schon, wie weit das mit der Demokratie irgendwie hin ist, wenn man in diesem Land nicht friedlich demonstrieren kann, ohne Angst zu haben, dass man verprügelt wird oder im Knast landet. Ist halt schwer. (FRANZISKA: 154-161)

Für SOPHIE war Polizeigewalt im Zuge einer Abschiebung ihres Mitschülers gar einer der Auslöser für ihr politisches Engagement. Besonders prägend war für FRANZISKA die Erfahrung, die sie und ihre Mistreiter*innen mit staatlichen Maßnahmen der Überwachung machten. Vergleichbare Erfahrungen wurden auch aus anderen Gruppen berichtet. An dieser Stelle ist es notwendig, noch einmal darauf hinzuweisen, dass für die vorliegende Untersuchung ausschließlich Einzelpersonen und Gruppen beforscht wurden, die offen auftreten und deren Plena öffentlich zugänglich sind. Es handelt sich um Kinder- und Jugendgruppen, die keine staatsfeindliche Ideologie propagieren oder konspirativ arbeiten. Dennoch berichten Schüler*innen von sogenannten Anwerbeversuchen des Verfassungsschutzes:

[E]s gab auch noch krassere Sachen, die bis hin zu überwachungsstaatlichen Maßnahmen eigentlich gegangen sind, dass man Leute auf der Straße offen angesprochen hat und nach Namen gefragt hat, sogar irgendwie Geld angeboten hat, von Mitarbeitern des Verfassungsschutzes, die sich sogar so vorgestellt haben. Unglaublich. (FRANZISKA: 871-879)

Diese Aussage lässt sich nicht verifizieren. Von vergleichbaren Erfahrungen berichteten allerdings Jugendliche aus verschiedenen Gruppen in Deutschland. Es ist festzustellen, dass aufgrund dieser und vergleichbarer Erfahrungen das Vertrauen von vielen Befragten in den Rechtsstaat gesunken ist. JAN beispielsweise erklärt das Vorgehen der Polizei auf friedlichen Schüler*innen-Demonstrationen als einen der „Hauptradikalisierungsfaktoren“ (JAN: 460). Und für JOHANNES ist „Polizeistaat“ (170) gar die erste Assoziation, wenn er an das politische System in der Bundesrepublik Deutschland denke. FRANZISKA beschreibt am Beispiel 
ihrer Festnahme bei einer Demonstration eindrücklich, warum sie dem Rechtsstaat nicht mehr vertraut.

\begin{abstract}
Absolut. Mich hat das total erschüttert. Ich hatte, auch wenn ich, wie ich zuvor schon gesagt habe, nicht die höchste Meinung vom deutschen Staat habe, immer noch ein Restvertrauen, dass zum Beispiel die Polizei mir eigentlich helfen wird oder so. Dass selbst, wenn die Polizei mal einen Fehler macht, dass es sich spätestens im juristischen Zusammenhang aufklären wird, dass man in Deutschland nicht einfach so ins Gefängnis gesperrt wird, dass man, wenn man im Gefängnis ist, aber menschlich behandelt wird. Aber ich hab das überhaupt nicht so erlebt. Ich wurde von Polizisten und Justizvollzugsbeamten beleidigt, gedemütigt, gezwungen mich auszuziehen, durfte, wie gesagt, keine Toilettentüren zumachen. Ich wurde in der Nacht jede halbe Stunde geweckt mit dem Vorwand, man müsse kontrollieren, dass ich mich nicht in der Zelle erhänge oder so, man hat zu essen nichts Vernünftiges bekommen. Man wurde angesprochen mit: Ey du Zecke, du Kröte, komm her, steh auf, komm mit, geh schneller, wir sind hier nicht im Hotel. Einfach total entwürdigend und das hat mich unfassbar schockiert. (FRANZISKA: 849-863)
\end{abstract}

Neben den Wechselwirkungen und den Interaktionen im sozialen Umfeld und den Konflikten mit staatlichen Institutionen und Akteur*innen birgt vor allem (Selbst)Überforderung der engagierten Schüler*innen ein enormes Frustrationspotential. Die Erfahrungsberichte von RONJA stehen stellvertretend für mehrere Beschreibungen dieser Art. Das politische Engagement ist für RONJA teilweise kräftezehrend und belastend. Sie hat sich daher professionelle psychologische Unterstützung gesucht.

Und ich selber habe dann vor ein paar Monaten die Hilfe von einer Psychotherapeutin gesucht, einfach so, um das so ein bisschen auszugleichen, weil mich dieses ganze Politische sehr beschäftigt. Einerseits die Themen, die halt so anstehen, dass ich manchmal nicht so gut damit klarkomme, wie blöd es um manche Dinge einfach steht oder mit dem Klimawandel, aber auch in der Flüchtlingsdebatte, was Menschen für Ansichten dazu haben. Einerseits damit, aber auch mit dem Organisieren. Das zum Ausgleich so (...3...) genau. (RONJA: 412-418)

Das Engagement führe bei RONJA zu Überforderung, Stresssymptomen und Panikattacken:

Aber andererseits kann ich auch sagen, dass es echt anstrengend ist. Super viel Zeit geht da rein, super viel Nerven. Es ist wirklich auch bei mir so, dass mich das auch psychisch sehr belastet. Ich hatte zu tun mit Panikattacken und ganz viel Stresssymptome. Und das war halt auch dann so Eigeninteresse, dass ich mir eine Psychotherapeutin gesucht habe. Aber so von der Schule oder so, da wäre das auf jeden Fall notwendig, dass man da drauf guckt. (RONJA: 1202-1208) 
Der Schule ist es, den Berichten von RONJA zufolge, ganz offenbar nicht gelungen, ihr politisches Engagement weder fachlich beziehungsweise fachdidaktisch noch pädagogisch zu begleiten.

Die Schüler*innen erleben aber im Kontext der politischen Aktion auch wertvolle Erfahrungen der Anerkennung. RONJA betont beispielsweise, dass sie große Unterstützung durch ihre Familie für ihr politisches Engagement erfährt. Sie verweist darauf, dass ihre Eltern zu den politischen Veranstaltungen kommen, die sie organisiert.

Da haben meine Eltern mich zum Beispiel ziemlich gut aufgefangen und unterstützen mich auf jeden Fall in dem, was ich mache, insofern, dass sie das gut finden. Und wenn ich irgendwas mache, kommen sie zu den Veranstaltungen und so weiter. (RONJA: 797-802)

Auch über Gleichaltrige, Mitstreiter*innen und Freund*innen erfahren die engagierten Schüler*innen immer wieder Bestärkung. Für LAURENZ sind die (neuen) politischen Beziehungen/Freundeskreise dabei besonders wichtig. „Ich habe viel mehr Kontakte, insgesamt mit politischen Kontakten und natürlich dann auch freundschaftliche Kontakte. Was auch ungemein bekräftigend ist", sagt LAURENZ (330-332) im Interview. Er erfährt im politischen Engagement viel positives Feedback, das ihn für seine weitere politische Arbeit motiviert.

[D]ann würde ich mich nicht als verdrossen bezeichnen, weil ich einfach jeden Tag, wenn ich mich in irgendeiner Form engagiere, merke, wie viel positiven Rückhall es gibt und wie viele Menschen auch mit mir zusammen daran arbeiten, daran Spaß haben und eben optimistisch sind, positive Ziele noch irgendwie erfassen können und eben nicht politikverdrossen sind. (LAURENZ: 228-232)

EMMA geht noch einen Schritt weiter und beschreibt das Wirken in der politischen Aktion auch als Sozialisationserfahrung, die es ihr ermöglicht habe, in einem sozialen Gefüge anzukommen. Sie betont dabei nicht nur Umgangsformen der Anerkennung und Wertschätzung, sondern vor allem die Möglichkeit, so sein zu können, wie sie ist.

Ich würde sagen, ich hatte vorher nicht so viele Freunde und es war immer dieser Kampf um soziale Strukturen für mich. Also ich würde mich auch eher als Außenseiter bezeichnen und dadurch, dass man dann halt im Endeffekt akzeptiert wird im Freundeskreis, so uneingeschränkt, also, verändert hat einen das auch. Also mich persönlich hat es auf jeden Fall verändert. Mir hat das gezeigt, dass es auch irgendwie anders geht und mir hat das eben auch Selbstbewusstsein gegeben auf jeden Fall. (EMMA: 592-598) 
Die politische Bezugsgruppe wird daher für die Teilnehmenden nicht nur politischer Sozialisationsraum, sondern vielfach auch sozialer Schutzraum und somit Raum der Anerkennung und Wertschätzung.

Aus der Perspektive der beobachtenden Teilnahme lässt sich dieses beschriebene Phänomen auch auf die anderen Gruppen übertragen. Die Jugendlichen praktizieren in den begleiteten Plenums-/Gruppensitzungen einen respektvollen und wertschätzenden Umgang miteinander. Sie nutzen Instrumente wie (doppelt)quotierte Redelisten, solidarische Kritik und non-verbale Kommunikationsmittel (Hände hoch für Zustimmung, Hände runter für Ablehnung). Uneingeschränkt beschreiben alle Befragten die Wirkung der Gruppe auf ihre persönliche Entwicklung als bereichernd. Diese konstruktive und selbst erarbeitete Diskussionskultur, die sich in den beforschten autonomen Jugendgruppen etabliert zu haben scheint, ermöglicht ihnen einen Lernprozess, der durch den konventionellen Unterricht kaum geleistet werden kann. Die Folge ist ein höheres Selbstbewusstsein der Befragten, auch gegenüber älteren Menschen und Autoritätspersonen (Kenner/Wohnig 2020, 115 f.).

Aber auch Lehrkräfte werden von den Befragten nicht nur als Hemmnis für selbstbestimmtes politisches Engagement von Schüler*innen dargestellt, sondern können dieses im Gegenteil auch nachhaltig fördern und damit zu bestärkenden Anerkennungserfahrungen führen. FRANZISKA beschreibt Erfahrungen mit Lehrkräften, die sie unterstützt haben und ihr Engagement wertschätzend begleitet haben. Für die politische Bildung ist dabei besonders relevant, dass offenbar keine dieser Lehrkräfte das Fach Politik unterrichtet. Das ist eine Erfahrung, die sich aus der Vielzahl der beforschten Jugendprojekte rekonstruieren lässt.

\begin{abstract}
Mmh (nachdenklich), also (..), ja es gab schon Lehrer, die auch immer mal wieder haben durchscheinen lassen, dass sie es gut finden. Also, zum Beispiel nach dem Abitur bin ich ja ein Jahr ins Ausland gegangen, nach Tansania. Und da hat mir eine Lehrerin so ein Referenzschreiben geschrieben, wo auch drinsteht, dass sie besonders an meiner Person hervorhebt, dass ich so engagiert bin und gesellschaftliches Interesse habe und dass sie das gut findet oder so. Oder meine Erdkundelehrerin war immer total beeindruckt, wie viele Sachen ich irgendwie weiß, aus anderen Zusammenhängen, und fand das richtig gut und hat mich eben auch bestärkt in den Sachen, die ich gemacht hab. (FRANZISKA: 946-954)
\end{abstract}

Sowohl Frustrations- als auch Anerkennungserfahrungen der Jugendlichen sind unter anderem auf Wir-ihr-Konstruktionen zurückzuführen. Auf dieses Phänomen, das sich aus den Berichten der Schüler*innen rekonstruieren lässt, wird im Folgenden genauer eingegangen. 


\subsubsection{Wir-ihr-Konstruktionen}

Die Beziehung zu den Mitschüler*innen wird bei politisch engagierten Jugendlichen zuweilen auf eine Probe gestellt. Schüler*innen, die sich Freiräume in der Schule für ihr politisches Engagement erkämpfen, sind oft mit Ablehnung durch ihre Altersgenoss*innen konfrontiert. Obwohl Konfliktfähigkeit als eine grundlegende Kompetenz der politischen Bildung hervorgehoben wird (u. a. Reinhardt 2018, 20-25), steht für die Schule zumeist der Wunsch nach einem konfliktfreien Raum im Vordergrund. Der Begriff „Schulfrieden“ schaffte es gar in mehrere Schulgesetze. Das Bedürfnis von Kindern und Jugendlichen, politische Konflikte zu thematisieren und auszuhandeln, stößt daher in der auf Konfliktfreiheit ausgerichteten Schule häufiger auf Widerstand.

[A]ls ich dann darauf geachtet habe, weil ich in der AG bin, habe ich ein bisschen auf mein Umfeld geachtet und habe gemerkt, dass in meinem sozialen Umfeld viel so etwas passiert [...], also die Meinungen selbst innerhalb der Klasse spalten, sogar so bei den Freunden spalten. Wir hatten letztens auch so einen Vorfall, bei dem die Hälfte der Klasse gegen uns war, weil wir der Meinung sind, es darf nicht in einem Erdkundebuch „Schwarzafrika“ stehen. [...] Also, wir haben schon echt Gegendruck, wir haben viele Leute, die gegen uns sind und viele Leute, die uns auslachen, weil wir so etwas machen. (ESRA: 212-222)

In ESRAs Aussage ist die besondere Sensibilität ihrer Gruppe für politische Themen auch in vermeintlich unpolitischen Momenten oder Schulfächern zu erkennen. Sie provozieren damit auch einen politischen Diskurs, politische Kontroversen und Konflikte. Aus der Perspektive der politischen Bildung ist dies grundsätzlich als willkommener Lernanlass einzuschätzen. ESRA führt aber weiter aus, dass diese politische Kontroverse immer häufiger auch in persönliche und emotionale Konflikte umschlägt. Sie berichtet davon, dass sie ausgelacht wurde und im weiteren Verlauf des Interviews schildert sie folgende Situation:

Mir wurde sogar schon mal ein Bein gestellt, als ich rausgegangen bin, weil viele gar nicht offen sind. Oder was heißt nicht offen, vielleicht finden sie es auch einfach uncool und wollen damit zeigen, dass sie cool sind, wenn sie anderen Leuten ein Bein stellen, die sich engagieren oder so. (ESRA 229-235)

Diese realen Erfahrungen der sozialen Exklusion in Schule oder Klassenverband befördern eine Wir-ihr-Konstruktion im Verhältnis zu den Mitschüler*innen. LEA konstatiert gar, dass diese Abgrenzung für sie zunächst ein Hemmnis war, sich politisch an der Schule zu engagieren: „Allerdings war es dann so, dass es eher als uncool galt, in eine $A G z u$ gehen. Und wenn man neu in so einer 
Klasse ist, will man dann halt nicht als ein totaler Nerd dastehen und dann in eine AG gehen. Deswegen habe ich es erst mal nicht gemacht. " (LEA: 387-390) Ihre Befürchtung, durch das politische Engagement Abgrenzungserfahrungen im Klassenverband und im sozialen Gefüge der Schule mit anderen Schüler*innen zu erleben, habe sich durchaus bestätigt. Die Vorwürfe ihrer Mitschüler*innen seien vielfältig und konzentrierten sich aber immer wieder auf die Unterstellung, den politisch engagierten Schüler*innen ginge es nur um Aufmerksamkeit:

Ja, ihr macht das nur für die Aufmerksamkeit, dass ihr euch für Politik interessiert. Oder: Ach, das sind schon wieder die von der Anti-Ra-AG. Ihr schon wieder, ihr müsst euch aber auch irgendwie über alles beschweren. So etwas interessiert doch niemanden, warum macht ihr das? Wir wollen was anderes machen. Und das interessiert uns nicht. (LEA: 466-476)

Auch ESRA bestätigt diese Erfahrungen. Im Unterricht, so sieht es die Mittelstufenschülerin, seien die Meinungen immer sehr gespalten, wobei sie das Gefühl habe, dass es in der Klasse oft gar nicht um den Inhalt gehe, sondern eher um die Lager ,die“" und ,wir".

Wobei es im Unterricht immer so ist, es fällt mir sehr auf, dass die Meinung sehr, sehr gespalten ist. Da gibt es immer die von der AG, die dann immer, also, fast immer dieselbe Meinung haben, was aber auch nicht immer stimmt, was aber auch nicht schlimm ist. Und dann gibt es die anderen, die sich oft immer gegen uns wenden. Und die gucken von einer ganz anderen Sichtweise, glaube ich, noch einmal. Vielleicht würde es ihnen sogar helfen, sich einfach mal in die AG zu setzen und zu gucken: Okay, es gibt vielleicht auch andere Sichtweisen. Sie sollten vielleicht auch mal überdenken, wie wir denken. (ESRA: 623-631)

Mit diesem Ausschluss ist auch der Begriff der Aussteiger*innen verbunden. ESRA besetzt diesen Begriff positiv: „Ich bewerte mich als ,Aussteiger“, weil ich es geschafft habe, nicht so wie alle anderen zu sein, nicht immer nur die Klamotten zu tragen, die andere anziehen, das zu machen, was mir gefällt. " (ESRA: 342-345) Ihre Mitschüler*innen, so erzählt es ESRA, nutzen diesen Begriff eher, um die Schüler*innen der Arbeitsgemeinschaft als Außenseiter*innen zu brandmarken.

Auch MICHAEL beschreibt vergleichbare Erfahrungen. Er organisierte eine Demonstration gegen Rassismus mit der SV und der Unterstützung mehrerer Lehrkräfte und der Schulleitung. Nur wenige Schüler*innen sollen sich, nach Aussagen MICHAELs, nicht der politischen Aktion angeschlossen haben. Diese hätten die Aktion dann ins Lächerlich gezogen. 
Also, die mitwollten, haben das eigentlich alle erlaubt bekommen, aber das waren dann Schüler, die einfach zu faul waren, oder einfach den Sinn der Demo nicht verstanden haben und das Ganze ins Lächerliche gezogen haben, die hiergeblieben sind. (MICHAEL: 236-239)

KATJA hat auch immer wieder die Erfahrung gemacht, dass politisches Engagement in der Schule auch von Mitschüler*innen eher als ,uncool“ eingeschätzt wird. Sie verweist daher auf die Notwendigkeit, Strategien zu entwickeln, wie mit jenen Mitschüler*innen umzugehen sei, die politisches Engagement in der Schule abwerten.

Und es braucht, glaube ich, auf jeden Fall (nachdenkend, langsam sprechend) zumindest ein, zwei Schüler, die auch wirklich Bock darauf haben und denen das auch wichtig ist. Und dann einfach, um Leute zu motivieren und zu zeigen: Hey, guck mal, so geht es auch. Ich habe da Bock drauf, aber ich habe trotzdem Spaß, so! Und dann muss man halt gut, glaube ich, mit den Leuten umgehen, die sagen: Was macht ihr denn für einen Scheiß? Also, das war bei uns halt auch gerade so in der Dreizehnten oder so, dass da Leute waren und gesagt haben: Ihr nervt mich mit eurem Karten verkaufen und euren Ansagen. Das man da irgendwie einen Weg findet, damit umzugehen, weil wenn man sagt: Halt die Schnauze! Dann bringt das halt nichts. Und wenn man nichts sagt, bringt das auch nichts und (..), ja, dass man sich damit irgendwie auseinandersetzt. (...) Ja. (KATJA: 880-890)

Der Konflikt mit der eigenen Peer-Group kann aber auch über das Verhältnis von Schüler*innen untereinander hinausgehen. So hat KATJA eine besondere Form der Ich-Ihr-Konstruktion durch ihr Engagement in der Antirassismus-AG erlebt. Anders als andere Schüler*innen, die vor allem in Konflikten mit ihren eigenen Mitschüler*innen gerieten, musste KATJA ihr Engagement nach außen rechtfertigen.

Ich wurde dann auch von Leuten teilweise, also von außerhalb der Schule, schief angeguckt, weil Antirassismus in der Schule, das ist ja ein bisschen, also in der Schule, naja, sie haben gesagt, dass ich ja auch richtig Politik machen könnte. (KATJA: 215218)

KATJA war vor ihrem selbstbestimmten politischen Engagement in der Schule schon lange außerschulisch politisch in autonomen Jugendgruppen aktiv. Ihren Schilderungen zufolge haben ihre Freund*innen, mit denen sie außerhalb der Schule politisch aktiv ist, ihr politisches Engagement im Kontext der Arbeitsgemeinschaft nicht erst genommen. Sie fühlte sich damit zu Rechtfertigungsstrategien genötigt. 
Den mit den Abgrenzungserfahrungen innerhalb der eigenen Peer-Group verbundenen Frustrationen im schulischen Alltag kann durchaus entgegengewirkt werden, indem Schule insgesamt als Raum des kontroversen politischen Diskurses verstanden wird. Dafür bedarf es auch eines anderen Selbstverständnisses von Lehrkräften. Die Wir-ihr-Konstruktion, die sich zu einem Selbstverständnis der politisch aktiven Gruppe zu entwickeln scheint, ist mehrdimensional. „Wir“ steht dabei für die eigene politische Gruppe, das „Ihr“ kann aber neben den Mitschüler*innen auch für die Lehrkräfte stehen.

Zumeist beschreiben die politisch aktiven Schüler*innen das Verhältnis zu ihren Lehrkräften als angespannt. Die Lehrkräfte distanzieren sich häufig von den politischen Projektideen der Schüler*innen. Beispielhaft hierfür kann ein Erfahrungsbericht angeführt werden, den RONJA aus einem Projekt schildert, das ihre Arbeitsgemeinschaft im Kontext der „Fridays for Future“-Bewegung etablieren wollte. Diese klimapolitische Jugendbewegung habe angeboten, eine Projektwoche zum Thema Nachhaltigkeit an der Schule zu organisieren. Sie fühlten sich dabei im Stich gelassen. Die Lehrkräfte machten, so berichtet es RONJA, unmissverständlich deutlich, dass sie sich daran nicht beteiligen würden. Es wäre schließlich ,ihr“ Projekt. (RONJA: 964-999) Auch SOPHIE sah Mitwirkung in der Schule immer eher als einen Prozess an, in dem Schüler*innen, die Verantwortung übernommen hätten, durch die Lehrkräfte instrumentalisiert worden seien. (SOPHIE: 444-457).

Dass sich politisch aktive Schüler*innen von ihren Lehrkräften eher emanzipieren, hat auch damit zu tun, dass sie immer wieder erleben, dass Lehrkräfte politisch relevantes Verhalten (beispielsweise Rassismus) ignorieren oder gar tolerieren. Beispielhaft sei hier ein Erfahrungsbericht von RONJA angeführt, der stellvertretend für verschiedene Berichte dieser Art steht:

Also, da bin ich echt total geschockt. Ich wünsche mir einfach andere Lehrer. Also, ich finde Schule eine Supersache, ich bin auch für die Schulpflicht meinetwegen so, ich finde es echt gut. Aber ich wünsche mir einfach andere Lehrer. Also, wie dumm manche einfach sind, das will ich gar nicht glauben. Also, dass man solche Leute einfach auf Kinder loslässt (..). Also, sie sind irgendwie auch nett, aber sie leben in ihrer kleinen Welt und kriegen nichts mit. Ich habe einen Lehrer gefragt, weil ich aus dem Bus heraus gesehen habe, dass er sich das Schild, das an der Bushaltestelle hing, durchgelesen hat, habe ich ihn mal gefragt, was er denn glaubt, was es ist, weil ich es blo $\beta$ von Weitem gesehen habe. Ich habe ihn gefragt, ob er mir sagen kann, ob es irgendwas Rechtes ist. Und der meinte „Nein, das sind Freiaktivisten, ganz normal.“ (...3...) Und wir haben übrigens dagegen Anzeige erstattet mit der Stadt zusammen. Aber davon erzähle ich meinetwegen auch gleich noch. (RONJA: 555-567) 
Die Tatsache, dass beispielsweise Rechtsextremismus durch eine Lehrkraft verharmlost wird oder wie in einem anderen Beispiel der Schriftzug „Refugees Welcome" auf einem T-Shirt von Lehrkräften als unerwünscht bezeichnet wird (BEN: 540-549), verdeutlicht, warum vor allem politisch engagierte Schüler*innen die Schule nicht als einen Schutzraum empfinden. Sie sehen darin ein strukturelles Problem der Schule.

Dabei sind Abgrenzungstendenzen nicht immer nur mit konkreten politischen Differenzen begründet. Vor allem die Abgrenzung zu den Erwachsenen als „die anderen“ begründen die Schüler*innen häufig damit, dass sie sich nicht ernst genommen fühlen. Wenn der Kampf um Freiräume und Handlungsräume mehr Energie bedarf als erwartet, bleibt oft kaum noch Kraft für die eigentliche politische Arbeit. Das „wir“ und „die anderen“ kann dann in bestimmten Fällen auch zu einem ,ich“ und „die anderen“ führen und zu einer besonderen Isolierung politisch aktiver Schüler*innen. So ergeht es JAN. Nach Konflikten mit seiner Schulleitung und seinen Mitschüler*innen fühlt JAN sich isoliert an der Schule. „Ich“ und „die anderen“, artikuliert in diesem Fall tatsächlich eine Konstruktion von ,ich“ gegen ,alle anderen“ und ,alle anderen“ gegen „mich“:

\begin{abstract}
Aber da zu dem Zeitpunkt hat es von den Schülern überhaupt keinen interessiert, die waren alle in Ferienstimmung, das hat mich halt total angekotzt an der Stelle. Es ging ja auch nur so um Unterstützungsmaßnahmen. Selbst wenn die jetzt keinen Bock gehabt hätten, jetzt selbst irgendwie einen Streik durchzuziehen oder so, dass man halt bei der Organisation von dem und dem vielleicht mithilft, oder eigentlich ging es schon nur darum, dass Klassensprecher und Klassensprecherinnen einfach ihre Aufgabe insofern wahrnehmen, dass sie die Informationen, die ich bei der Klassensprecherversammlung auch rüberbringe, dass sie die auch in die Klassen weitergeben und da ist es an dieser Stelle schon gescheitert, weil Leute einfach Klassen- und Kurssprecherinnen werden, weil das kein anderer machen wollte oder, keine Ahnung. Die Leute haben keinen Bock und gehen zum Beispiel auch überhaupt nicht zu den Klassensprecherversammlungen und dadurch habe ich mich an der Stelle so ein bisschen isoliert gefühlt, würde ich sagen. Und das war bei mir auf jeden Fall ein Faktor zur Radikalisierung. (JAN: 502-516)
\end{abstract}

Die Politisierung seiner Rolle als Schulsprecher führte zu einer Vereinsamung. Seine eigene Rolle, auch seine individuelle Verantwortung für diesen Prozess reflektiert er dabei kaum. Es gibt aber offenbar auch keine Möglichkeit mehr, ihn über pädagogisches Fachpersonal zu erreichen. Die Schule als Sozialisationsinstanz, aber auch als pädagogisch begleiteter Raum, hat den Zugriff auf diesen politisch interessierten und politisch aktiven Schüler verloren. 


\subsubsection{Politische Persönlichkeit und Selbstwirksamkeit}

\subsection{Positive Auswirkung auf Persönlichkeit und Selbstwirksamkeit}

In dem folgenden Unterkapitel wird der Frage nachgegangen, welchen Einfluss die Erfahrungen aus selbstbestimmter politischer Aktion auf das Selbstwirksamkeitsempfinden der interviewten Schüler*innen hat. Das diesen Überlegungen zugrunde liegende Verständnis von Selbstwirksamkeit geht auf die Arbeit von Albert Bandura (u. a. Bandura 1999) zurück, der zu den führenden Psycho$\log$ *innen in der zweiten Hälfte des 20. Jahrhunderts zählt und wichtige Arbeiten im Zusammenhang von Lernpsychologie und sozialem Lernen veröffentlicht hat. Kern des Selbstwirksamkeitskonzepts nach Bandura ist die Fähigkeit, an sich selbst zu glauben, Vertrauen in die eigenen Kompetenzen zu entwickeln und so neue und schwierige Anforderungssituationen bewältigen zu können. Die Theorie geht davon aus, dass der Erfolg von Handlungen und die tatsächlichen eigenen Fähigkeiten von der subjektiven Einschätzung gesteuert werden, vor allem durch die Ergebniserwartung und die Selbstwirksamkeitserwartung. Sie bezeichnet dabei die Einschätzung der eigenen Wirksamkeit bzw. die Überzeugung von den eigenen Fähigkeiten und Kompetenzen. Die Selbstwirksamkeitserwartung ist dabei abhängig von der individuellen Einschätzung einer Herausforderung der zu bewältigenden Situation (level), dem Ausprägungsgrad der Selbstwirksamkeit (strength) und dem Verallgemeinerungsgrad der Selbstwirksamkeitseinschätzung (generality). Die Überzeugung der eigenen Handlungsmächtigkeit begünstigt politische Lernprozesse (Bandura 1977).

Die positiven Einflüsse der Erfahrungen aus den selbstbestimmten politischen Aktionen der interviewten Schüler*innen wurde induktiv kodiert. Dabei ließen sich folgende Kategorien herausarbeiten:

- sinnstiftender Kern des eigenen Handelns bzw. die Überzeugung, einen wesentlichen Beitrag zu leisten

- selbstbewusst zur eigenen (politischen) Identität stehen

- Aktion als Motivation und Bestärkung für eigenes Handeln begreifen

- Entwicklung konkreter Fähigkeiten

Als negative Einflüsse auf Selbstwirksamkeitserwartungen der Schüler*innen ließen sich folgende Kategorien rekonstruieren:

- Unzufriedenheit mit sich selbst und der Welt

- Überlastung führt zu Selbstzweifel

- Radikalisierung durch Vereinsamung 
Unabhängig von den jeweiligen positiven und negativen Auswirkungen des politischen Handelns auf die Selbstwirksamkeitserwartungen der Schüler*innen ließ sich aus den Interviews herausarbeiten, dass das selbstbestimmte politische Handeln immer nachhaltig Einfluss auf den Alltag der jungen Menschen genommen hat. Beispielhaft kann hier eine Aussage von KATJA angeführt werden, die beschreibt, wie ihr politisches Engagement auch prägend dafür gewesen sei, wohin und wie sie in den Urlaub fahre, wie sie sich ernähre und was sie in ihrer Freizeit mache.

\begin{abstract}
Ich glaube, das verändert irgendwie alles. Also, sowohl das Essen als auch das, keine Ahnung, wo man in Urlaub hinfährt. Oder, also, es ist, glaube ich, auch möglich, dass es nichts verändert, wenn man das wirklich will. Aber ich glaube, eigentlich verändert das ziemlich viel. Wobei es verändert nie nichts. Dass es weniger verändert, aber ich glaube eigentlich ist das (...), ja keine Ahnung. Ich habe aufgehört Ski zu fahren, weil ich dachte, das ist irgendwie doof für die Umwelt und so etwas, also, es ist schon ziemlich viel, was dadurch verändert wird. (KATJA: 776-783)
\end{abstract}

Die Jugendlichen begreifen ihre politische Arbeit als Teil ihrer Persönlichkeitsbildung. Dabei betonen sie vor allem den sinnstiftenden Charakter des politischen Handelns. „Das ist Teil meiner Identität, macht mich aus, macht mir Spaß. Ich liebe meine Arbeit total. " (FRANZISKA: 840-841) Diese Aussage von FRANZISKA symbolisiert den Wert, den alle interviewten Schüler*innen ihrem politischen Engagement beimessen. Die Jugendlichen empfinden ihre politische Arbeit als Chance zur Selbstentfaltung. Die politische Arbeit stärkt das Selbstwirksamkeitsempfinden der Interviewten dadurch, dass sie ihre eigenen Stärken und Schwächen identifizieren können. So betont LAURENZ: „Mir macht es Spaß, was ich mache, eben auch weil ich es optimistisch sehe. Ich glaub, dass ich recht gut bin, in dem was ich mache. Also, ich bin darin zum Beispiel viel besser als in Chemie (lacht). Also, das bedeutet ja auch, dass ich ja auch Erfolge dabei habe, Erfolgsgefühle dabei habe. "(LAURENZ: 293-298) Diese Erfolgserlebnisse und Bestätigungen, die Suche und das Finden der eigenen politischen Identität und der individuellen Stärken prägen nach ihrer eigener Einschätzung die Persönlichkeit der politisch aktiven Schüler*innen. Durch ihr politisches Engagement steigt ihr Selbstbewusstsein. Sie lernen, dem Anpassungsdruck zu widerstehen, und spüren subjektiv die Entwicklung einer eigenen politischen Persönlichkeit.

Alle interviewten Jugendlichen verweisen darauf, dass es ihnen besonders wichtig sei, mit dem eigenen politischen Engagement einen Beitrag für die Gesellschaft zu leisten. LEA sagt, es gebe ihr ein gutes Gefühl, etwas getan zu haben und einen kleinen Beitrag zur Veränderung leisten zu können. Es ist den politisch Aktiven wichtig, etwas anzustoßen, etwas in Bewegung zu bringen: „Und 
das ist irgendwie für mich selbst wichtig, so das Gefühl, dass ich was verändern kann. Und etwas verändert habe schon in der Vergangenheit. “ (LEA: 856-863) Die politische Arbeit ist sinnstiftend und diese Sinnstiftung vermittelt ihnen zugleich Sicherheit. RONJA formuliert dieses Gefühl wie folgt: „Also, ich bin sicherer geworden in manchen Dingen. (...3...) Fühle mich auch in mancher Hinsicht wichtig, also nützlich. “ (RONJA: 1201-1202) Unabhängig davon, ob sich die Kinder und Jugendlichen in der Schule oder außerhalb der Schule politisch engagieren, ähneln sich die Beschreibungen, welchen Stellenwert ihr politisches Handeln auf ihr Selbstwertgefühl hat. Ihr politisches Handeln stärkt ihr Selbstbewusstsein. Auch einzelne Aktionsformen und Ereignisse können dabei prägend sein. MICHAEL beschreibt, welche Gefühle ihn nach einer Demonstration gegen Rassismus bewegten. Es war das erste Mal, dass er eine Demonstration organisiert hat:

Also, in der Vorbereitung haben wir uns als Gruppe, also haben wir auch am Ende gesagt, wir haben uns alle, also ich persönlich mich ziemlich stark gefühlt, weil wir uns einfach engagieren und aufgestanden sind, irgendwas getan haben und nicht nur rumsitzen und nichts tun. Das hat sich, ich kann das Gefühl gar nicht beschreiben, es hat sich einfach gut angefühlt, etwas zu tun. (MICHAEL: 256-260)

Allerdings verbinden die Interviewten Selbstwertsteigerung nicht nur mit Erfolgsmomenten. FRANZISKA sieht vor allem eine Stärke in der Bewältigung von Frustrationserfahrungen sowie im Umgang mit Rückschlägen (FRANZISKA: 561-570) und in der gemeinsamen Bewältigung der politischen Herausforderungen: ,Und das hat mir total Kraft gegeben auch persönlich und mich persönlich total weiter gebracht, zu sehen, dass es Menschen gibt, die etwas verändern wollen. “ (FRANZISKA: 485-487). Die politisch aktiven Jugendlichen sprechen dem Engagement in der Gruppe einen besonders hohen Wert bei. Die kollektive Partizipationserfahrung hilft ihnen gegen Ohnmachtserfahrungen. Exemplarisch sei hier eine Aussage von KATJA angeführt, die beschreibt, dass das Gefühl, nicht allein zu sein mit seinem Engagement, sie in der Überzeugung stärke, gemeinsam mit den anderen einen Beitrag leisten zu können.

Und wenn man dann das Gefühl hat, es sind auch Leute da, die das Gleiche versuchen, dann hat man gleich das Gefühl, dass es doch nicht ganz so aussichtlos ist, weil man eben nicht alleine gegen alle so, und das ist schon ganz okay (lacht). (KATJA: 467-470)

Dennoch ist sich KATJA auch des emotionalen Dilemmas bewusst, in dem sie durch ihr Engagement steckt. Sie weiß, dass sie die Welt nicht verändern kann, aber durch ihr politisches Engagement ist sie zu der Überzeugung gelangt, dass 
sie zumindest Menschen zum Nachdenken anregen und vielleicht für den Einzelnen etwas erreichen könne (KATJA: 452-459). Ihre Rolle als politisches Subjekt in einer komplexen politischen Welt scheinen die Jugendlichen durch ihr politisches Engagement zu finden. Wenngleich sich die politisch aktiven Schüler*innen zumeist einig sind darüber, dass ihnen, auch aufgrund ihres Alters, zu wenig zugetraut wird, haben sie erkannt, dass sie mit ihrem Engagement etwas erreichen können. Die Komplexität politischer Themen birgt allerdings auch die Gefahr, die Schüler*innen zu überfordern oder Momente der Desillusion zu befördern. So betonen sie doch immer wieder, dass sie die Wirksamkeit ihrer Arbeit erleben, und sei es nur die Tatsache, dass sie und ihre Mitstreiter*innen dem Gefühl der politischen Ohnmacht etwas entgegensetzen können:

Dann kann ich aber wenigstens etwas für mich machen, selbst wenn es für nicht viele andere etwas verändert. Irgendetwas verändert es und wenn es auch nur in meiner persönlichen Wahrnehmung ist, dass ich die Struktur nicht mehr als so mächtig wahrnehme, dann hat das schon irgendwas gemacht. (JOHANNES: 609-613)

Inwiefern das Gefühl von Selbstwirksamkeit bei den Kindern und Jugendlichen gestärkt wird, lässt sich in dem Untersuchungsfeld vor allem daran ableiten, welchen Einfluss das politische Engagement auf die Bereitschaft und Befähigung zur Teilhabe an politischen Diskursen hat. Hier lässt sich in besonderer Weise der Einfluss von Partizipationserfahrungen rekonstruieren. Das politische Engagement in den selbstorganisierten Arbeitsgemeinschaften oder im Kontext außerschulischer politischer Aktionen nimmt den Interviewten nach eigenen Erfahrungsberichten die Unsicherheit im politischen Meinungsaustausch. MICHAEL beschreibt beispielweise, dass er erst durch das politische Engagement den Mut gefunden habe, Menschen anzusprechen und das Politische zum Thema zu machen (MICHAEL: 311-315). Er beschreibt diese Erfahrung als einen „Reifeprozess“":

[S]ich in Diskussionen begeben, auch mit Leuten aus dem alläglichen Umfeld darüber reden, Positionen allgemein vertreten, sich dafür kritisieren lassen, darüber reflektieren, so einen Reifeprozess auch dabei durchmachen. (BEN: 23-26)

Auch LEA sagt, sie sei durch ihr Engagement in der politischen Gruppe selbstbewusster geworden und traue sich erst seit diesem Engagement, ihre Meinung auch in anderen Kontexten zu äußern und zu vertreten.

Also, ich glaube schon, dass es irgendwie was an mir verändert hat. Weil ich früher eher so unscheinbar war. Und wenn jemand etwas gesagt hat, was mir nicht gefallen hat, dann war es halt okay. Vor allem in der 7. Klasse, als dann da ganz neue Leute 
waren, habe ich mich gar nicht getraut, irgendwas zu sagen oder so. Aber seit ich mich in der AG engagiere, ist es mehr so, dass ich dann auch sage, wenn mir was nicht gefällt oder ich was zu einer Meinung zu sagen habe. Dann mache ich das. Und das war halt früher nicht unbedingt so. Ich war nicht unbedingt schüchtern, aber ich war vorsichtig, dass ich dann nicht irgendwie hinterher von allen voll gehasst werde, dass ich eine andere Meinung als die vertrete. (LEA: 662-671)

Diese Erfahrungen legen für die interviewten Schüler*innen nicht nur den Grundstein für eine politische Positionierung, sondern helfen ihnen auch, diese zu artikulieren und $\mathrm{zu}$ verteidigen. Auch HELGE schildert eine positive Persönlichkeitsentwicklung, die er auf sein selbstbestimmtes politisches Engagement zurückführt. Er ist überzeugt, dass er heute selbstbewusster sei und auch politische Positionen eher öffentlich vertrete als früher und sich nicht so schnell entmutigen lasse.

Mittlerweile kann ich mich auch mit Lehrern oder anderen Schülern soweit darüber unterhalten, dass ich weiß, ich vertrete einen Standpunkt, den ich auch belegen kann und womit ich mich auch gerne auseinandersetze. Den Mut hätte ich, glaube ich, vor einem Jahr noch nicht gehabt. [...] Ich traue mich viel mehr, meine Meinung auch offen kundzugeben und auch mich damit auseinanderzusetzen, wenn vielleicht nicht jeder derselben Meinung ist, auch im Hinblick auf ältere Menschen oder Lehrer oder Erziehungsberechtigte. Ich bin viel mutiger und selbstbewusster geworden, das auch $z u$ vertreten und mich auch dafür einzusetzen, und nicht gleich zu sagen, wenn es auf Kritik stößt: Ach, ich höre jetzt auf, weil vielleicht andere, die sich damit länger auseinandersetzen, eine andere Meinung haben. (HELGE: 286-332)

Auch ESRA berichtet davon, dass sie durch ihr Engagement selbstbewusster geworden sei und sich eher traue, Missstände offen anzusprechen und Probleme $\mathrm{zu}$ benennen, in der Schule aber auch in ihrem Freundeskreis und der Familie (ESRA: 276-282). Sie hat sich lange eher als Mitläuferin verstanden. Erst durch ihr politisches Engagement habe sie eine politische Identität entwickelt, die es ihr ermögliche, selbstbewusst ihre politische Position mitzuteilen und $\mathrm{zu}$ vertreten.

Früher war ich auch eigentlich eher so, also, ich selbst habe mich zum Beispiel auch verändert. Ich kann sagen, dass ich früher eher so ein Mitläufer war und ich jetzt komplett selbstbewusst bin, und ich will jetzt nicht sagen „Aussteiger“, aber dass ich sage, also ich habe meine eigene Meinung und ich kann auch meine eigene Meinung anderen Leuten mitteilen. (ESRA: 315-319) 
Die politische Aktion fördert aber nicht nur die Selbstwirksamkeit in Bezug auf den politischen Meinungsaustausch. Vielmehr befähigt das politische Handeln auch die befragten Jugendlichen dazu, die vermeintlich festen politischen Strukturen aufzubrechen. JOHANNES beschreit das wie folgt:

Als Kind oder vielleicht auch als Jugendlicher wirken die etablierten Machtstrukturen so überwältigend. Die waren immer schon da, die werden immer da sein. Du kleiner Mensch kannst nichts machen. Und diese Projekte beweisen, dass das geht. Man ist da, man macht irgendwas. Etwas passiert und wenn es nur eine winzig kleine Veränderung ist, sie findet statt. Und das nimmt ganz viel Angst und Unsicherheit eben vor dem System und vor der Welt, dass man eben merkt, man kann selbst etwas auf die Beine stellen. Feste Strukturen sind nicht so fest, wie sie zuerst wirken. Das ist mir dadurch noch einmal wirklich klar geworden. (JOHANNES: 571-578)

Mit ihrem politischen Engagement halten die Schüler*innen den Ohnmachtserfahrungen etwas entgegen. Hilfreich sind dabei die scheinbar durchaus euphorisierenden Gefühle, die die Jugendlichen mit ihren politischen Aktionen verbinden. Eindrücklich beschreibt das MICHAEL, am Beispiel der AntirassismusDemonstration mit seinen Mitschüler*innen, die er maßgeblich organisiert hat:

\begin{abstract}
Also, die ganze Menschenmasse zu sehen, die dann auch von den jüngeren Schülern mitgelaufen sind und alles. Dass wir das in dieser kurzen Zeit geschafft haben, die ganzen Schilder und Plakate, die gebastelt wurden. Unser Ruf war ,Wir sind mehr“. Dass wir das alle gerufen haben immer, die ganze Zeit und dass alle so mitgemacht haben, ja, das war unglaublich. Das wir das hier als kleine Schule in so einem kleinen Dorf hingekriegt haben. So etwas hat es hier vorher auch noch nicht gegeben in diesem kleinen Ort, ne Demo. (MICHAEL: 268-274)
\end{abstract}

Für MICHAEL war die Organisation der Demonstration die erste politische Aktion und sie hat ihn, sein politisches Bewusstsein und sein Selbstwirksamkeitsempfinden nachhaltig positiv geprägt. Er beschreibt beispielsweise, dass er sich auch vor der Demonstration nicht als rassistisch bezeichnet hätte. Aber erst durch die Auseinandersetzung mit dem Thema in Vorbereitung und Durchführung der politischen Aktion sei er dafür sensibilisiert worden (MICHAEL: 296-299). Für die Frage nach dem Selbstwirksamkeitsempfinden ist aber entscheidender, dass er jetzt auch bereit ist, aktiv gegen Rassismus vorzugehen, auch im Alltag. „Wenn Leute das machen [rassistische Äußerungen], spreche ich die an und sag: Hey, muss das sein? Oder ich frage sie, was sie damit bezwecken wollen. Also, es hat in mir ziemlich viel verändert, einfach weil ich mir vorher nie so Gedanken darüber gemacht habe. " (MICHAEL: 296-301) 
Die Ausprägung der individuellen Selbstwirksamkeitseinschätzung ist eng verbundenen mit den individuellen Fähigkeiten, die es Menschen ermöglichen, sich neuen Herausforderungen zu stellen. Daher ist es für die vorliegende Untersuchung von besonderer Bedeutung, inwiefern sich aus den Erfahrungsberichten der politisch engagierten Schüler*innen eine unmittelbare Verbindung zwischen politischem Engagement und konkreten Fähigkeiten rekonstruieren lässt, die auf eine Steigerung der Selbstwirksamkeitserwartungen schließen lassen. Neben den bereits strukturiert in den Abschnitten 4.3.1 bis 4.3.4 herausgearbeiteten politischen Selbstbildungserfahrungen lassen sich noch weitere Fähigkeiten hervorheben, die Rückschlüsse auf das subjektive Selbstwirksamkeitsempfinden der Interviewten zulassen.

Die Jugendlichen betonen, dass sie durch das politische Engagement Vertrauen in ihre individuellen politischen Artikulationsfähigkeiten gewonnen hätten. So erklärt KATJA beispielsweise, dass sie durch ihr politisches Engagement und durch die Übung im politischen Diskurs sprechfähiger geworden sei. „Ich glaube, ich kann wesentlich besser vor Leuten reden und mit Leuten diskutieren, weil ich das oft mache, als ich das können würde, wenn ich das eben nicht durch den politischen Kontext machen würde. " (KATJA: 783-787) Auch in Bezug auf den Verallgemeinerungsgrad ihrer Selbstwirksamkeit lassen sich Schlüsse aus den Erfahrungsberichten der Schüler*innen ziehen. Sie verweisen immer wieder darauf, dass die politische Arbeit auch dazu führe, dass sie in anderen Lebensbereichen effektiver seien: „Also, ich glaube, mittlerweile kann ich da auch effektiver und effizienter meine Arbeit investieren “, führt beispielsweise RONJA (914-916) an. Und sie bemerkt außerdem, dass sie gelernt habe, besser mit Stress umzugehen (RONJA: 1208-1210). Das sagt auch SOPHIE in ihrem Erfahrungsbericht und ergänzt, dass sie gelernt habe, auf sich und ihre eigenen Bedürfnisse zu achten und diese nicht immer hinten anzustellen.

Aber keine Ahnung, was aber auch wieder eine wichtige Erfahrung ist, weil man auch lernen muss, irgendwie zu schauen, dass man sich sowohl irgendwie politisch engagiert oder für eine Sache engagiert, aber auch nicht vergisst, auf sich selbst zu schauen (lacht). Und dann ab und zu doch auch mal die Sachen macht, die man eben gerne macht, aber auch gerne außerhalb dieser Gruppe. (SOPHIE: 394-399)

Durch das Engagement hat sich das Selbstwirksamkeitsempfinden der Schüler*innen gesteigert. Und teilweise wird das auch klar benannt. So erklärt MICHAEL, dass er „ein bisschen selbstbewusster geworden“ (MICHAEL: 306) sei. Auch FRANZISKA formuliert das so deutlich und ergänzt, dass sie sich durch 
ihre Erfahrungen in der politischen Aktion nicht mehr klein, hilflos oder ohnmächtig fühle. Auch dieses Phänomen lässt sich in mehreren Interviews nachzeichnen, die folgenden Aussagen stehen stellvertretend dafür:

Ja. Ich habe auch im persönlichen Rahmen Selbstbewusstsein gewonnen dadurch. Und auch mehr Möglichkeiten gewonnen, mein eigenes Leben zu gestalten. Ich hatte davor so eine passive Einstellung zu allem. Ich war halt auch im Gymnasium so, man macht halt, man kommt in die nächste Klasse, man kommt in die nächste Klasse und so. Aber auf einmal habe ich auch angefangen, Sachen, die mich in meinem ganz konkreten Umfeld stören, einfach anzusprechen. Zum Beispiel, der eine Mathelehrer hat uns immer viel zu viele Hausaufgaben aufgegeben. Früher wäre mir nie eingefallen zu sagen: „Hören Sie mal, Herr Soundso, das ist jetzt wirklich nicht angemessen“. Und mit dem darüber eine Diskussion anzufangen, das hätte ich mich nie getraut. Aber wenn man sich in einem Umfeld bewegt, in dem man dauernd Diskussionen anfängt und in dem das auch zu Erfolg führt, in dem man auch wahrgenommen wird als Person und nicht als jemand, der eigentlich noch zu klein ist oder so, um seine Meinung zu vertreten, dann gewinnt man auf jeden Fall auch Möglichkeiten, sein eigenes Leben so zu gestalten, dass es einem besser gefällt. Das war auf jeden Fall gut für mich. (FRANZISKA: 535-549)

Abschließend sei darauf verwiesen, dass selbst die Wirkung des politischen Engagements von einigen Schüler*innen auf einer abstrakten Ebene reflektiert wird. Dies wird in den Aussagen von LAURENZ deutlich, der zunächst darauf eingeht, wie gut es im tue, dass es in der Gruppe ein solidarisches Miteinander gebe (LAURENZ: 299-303). Auf die Frage, ob das auch Antrieb für seine Arbeit sei, reagiert er wie folgt:

Ja, das auch. Das ist halt ein Teil davon. Also, nicht das Einzige. Wenn es das Einzige wäre, dann würde ich das wieder als egoistisch irgendwo charakterisieren. Und es würde dann ja letztendlich darauf hinauslaufen, dass es dann, wenn ich einen Pro-Flüchtlings-Protest irgendwo organisiere, dass ich dann keine Politik mehr mit Flüchtlingen machen möchte, oder so, sondern einfach für Flüchtlinge, um mich selbst irgendwie zu bereichern. Also das ist überhaupt nicht das, was ich dabei sehe. (LAURENZ: 309-315)

Dieses abstrakte Reflexionsniveau ist bemerkenswert. Aber das politische Engagement hat nicht nur im positiven Sinne Auswirkung auf Persönlichkeit und Selbstwirksamkeit der politisch aktiven Jugendlichen. Im Folgenden werden exemplarisch einige negative Faktoren rekonstruiert. 


\subsection{Negative Auswirkung auf Persönlichkeit und Selbstwirksamkeit}

Die negativen Auswirkungen selbstbestimmter politischer Aktionen auf das Selbstwirksamkeitsempfinden politisch aktiver Jugendlicher wird im Folgenden an drei Fallbeispielen rekonstruiert. Deutlich wird dabei vor allem die mit dem selbstbestimmten politischen Engagement womöglich verbundene Ambivalenz zwischen dem Gefühl, etwas bewegen zu können, und dem Gefühl der Ohnmacht und Hilflosigkeit. Im Folgenden wird exemplarisch aufgezeigt, wie sehr dieser innere Widerspruch die Schüler*innen beschäftigt.

Das selbstbestimmte politische Engagement, aber vor allem auch die damit verbundene Auseinandersetzung mit politischen Macht- und Herrschaftsverhältnissen sowie die Bewusstwerdung der eigenen politischen Machtlosigkeit führt bei einigen interviewten Schüler*innen auch zu einem Gefühl der Unzufriedenheit. Auf die Frage, ob und wann er mit einem Tag als politischer Aktivist zufrieden sei und ein gutes Gefühl habe, antwortet BEN: „Selten oder gar nicht. Das ist für mich sehr, sehr schwierig, weil es fällt mir sehr schwer mich mit kleinen Dingen zufrieden zu geben. Also, das hängt noch mit einigen Sachen zusammen, aber ich habe grundsätzlich Probleme damit. " (BEN: 331-333) Auch wenn es immer wieder positive Erfahrungen gibt, scheint BEN doch zumeist desillusioniert. Die großen Ziele seien unerreichbar. Aber er geht davon aus, dass das eher ein Mentalitätsproblem sei. Dass er auch diese Erfahrungen reflektiert, dokumentiert die folgende Aussage:

Aber am Ende, also irgendwie meistens gehe ich dann abends ins Bett und denke mir so, also jetzt haben wir fünf neue Leute im Plenum, was eigentlich eine gigantisch große Zahl ist, wenn man sich überlegt, dass sie vielleicht auch nächste Woche wiederkommen, dann auch langfristig aktive Mitglieder bleiben, bei der Gruppe. Dann sollte mich das eigentlich total freuen, das weiß ich eigentlich auch. Aber trotzdem am Ende habe ich immer so das Gefühl, ja, also damit haben wir dem jetzt wohl keinen endgültigen Schlag versetzt. Also irgendwie, das frustriert mich langfristig persönlich auf jeden Fall. Obwohl das auch eine Mentalität und Einstellung ist, wo ich auch versuchen will, an mir zu arbeiten. Ich will ja gar nicht sagen, dass ich irgendwie am Ende bin. Ich bin ja auch erst ein Jahr dabei. Das bedeutet eigentlich, dass ich an meiner Mentalität ein bisschen arbeiten muss. Ich muss ein bisschen gucken, dass ich etwas positiver werde. Es ist auch teilweise echt destruktiv, wenn teilweise, keine Ahnung, wie ich drauf bin, so das Gefühl habe, wir kriegen jetzt sowieso nichts hin, egal wie gut wir uns da reinhängen, und egal, wie viel wir uns vorbereiten. (BEN: 338-353)

Die Ambivalenz zwischen dem gesteigerten Selbstwirksamkeitsempfinden und dem Gefühl von Ohnmacht und Unzufriedenheit mit den politischen Verhältnissen und der eigenen Machtlosigkeit ist sinnbildlich für die Erfahrungswelten der jungen Menschen, die für diese Studie interviewt wurden. Ihr Antrieb ist der Wunsch 
nach Veränderung und die kritisch reflektierte Auseinandersetzung mit bestehenden Macht- und Herrschaftsverhältnissen sowie der bestehenden Ungerechtigkeit. Die damit verbundenen inneren und äußeren Kämpfe führen sie an die Grenzen ihrer Belastbarkeit. Das Identifizieren des Politischen in nahezu allen, auch alltäglichen Erfahrungen führt teils zu einer Überwältigung. RONJA beschreibt, wie die Politisierung dazu beigetragen habe, dass sie nicht mehr abschalten könne:

Weil ich doch merke, dass umso mehr ich mich engagiere, umso schlimmer fallen mir die Dinge auf, umso mehr fällt mir auf, umso schlimmer habe ich ein Bild von der Welt. Oder Situationen, von denen ich weiß, umso schlimmer finde ich es im Grunde. Und umso mehr ich mich damit beschäftige, umso mehr werde ich dadurch ernüchtert, durch solche Aussagen. (RONJA: 1210-1214)

Das belaste die Schülerin. Dieses Phänomen der Überforderungen wird dadurch verstärkt, dass die Schülerin, nach eigenen Aussagen, in der Bewältigung dieser Stresssituationen von pädagogischen Kräften keine Unterstützung erfährt. Mehrere Schüler*innen berichten, dass sie sich von den Lehrkräften zwar keine politische Unterstützung wünschten, aber eine pädagogische Begleitung.

Diese pädagogische Begleitung des politischen Engagements kann möglicherweise auch dabei helfen, Radikalisierungs- und Verinselungseffekten entgegenzuwirken. JAN, der sich eher als Einzelkämpfer beschreibt, antwortet auf die Frage, ob er das Gefühl habe, dass ihn Unterstützung vonseiten der Lehrkräfte hätte davor bewahren können, sich in seinem politischen Engagement zu radikalisieren, wie folgt:

Dann hätte ich mich ganz einfach nicht radikalisiert. Das ist so. Moment, was wollte ich jetzt sagen? Es ist natürlich so, wenn man merkt, dass man auch relativ wenig Unterstützung hat und dann so langsam auch dieses Einzelkämpferding kommt, also gerade zum Beispiel an meiner Schule, wo ich die Streiks initiieren wollte und dann die Leute zumindest zu dem Zeitpunkt noch nicht mitgezogen sind, ja das man versucht, diese fehlende Unterstützung, also man hält ja seine Sache für richtig und möchte die auch umsetzen, weil man sie eben richtig findet. Und wenn da halt die Unterstützung fehlt, dann versucht man das natürlich auszugleichen durch stärkere Aktionsformen und dadurch kommt es natürlich zu einer Radikalisierung. (JAN: 472-481)

Jan hat sich an seiner Schule, nach eigener Aussage, in der Zeit als Schulsprecher isoliert gefühlt. Er wollte diese Rolle politisch ausfüllen und erhielt dafür weder von Lehrkräften noch durch die Schüler*innen Unterstützung (JAN: 502516). Pädagogische Begleitung muss in diesem Fall nicht bedeuten, BEN in seinem politischen Engagement zu bestärken. Es könnte auch bedeuten mit ihm gemeinsam aufzuarbeiten, warum er für seine Arbeit keine Unterstützung erfährt. 


\subsection{Diskussion und Einordnung der Untersuchungsergebnisse}

Grundlage für die strukturierte Analyse der Interviews und damit der Rekonstruktion von Bildungserfahrungen der politisch aktiven Schüler*innen waren unter anderem die Erkenntnisse aus den Diskussionen zu Kompetenzmodellen im Feld der Politikdidaktik (u.a. Behrmann u.a. 2004; GPJE 2004; Detjen u.a. 2012; Reheis u.a. 2016; Oeftering 2016). Diese konzeptionellen Überlegungen wurden in Abschnitt 2.2 „Politische Bildung - Bewusstsein, Erfahrung und Emanzipation“ kritisch reflektiert und eingeordnet. Für die vorliegende Untersuchung wurden dabei vier zentrale Felder identifiziert, die politische Bildungsprozesse markieren: (1) Analysefähigkeit und politische Orientierung, (2) Perspektivenwahrnehmung, (3) Urteilsbildung und Kritik sowie (4) Partizipation und Konfliktfähigkeit. Diese Kompetenzfelder politischer Bildung dienten im Sinne einer deduktiven Strukturierung des Materials als Orientierung für die Rekonstruktion der Bildungserfahrungen von Jugendlichen, die selbstbestimmt und selbstorganisiert politisch aktiv sind. Darüber hinaus wurde als induktiv ermittelte Hauptkategorie (5) das Politische Selbstkonzept als prägendes Element identifiziert. Im Folgenden werden die Ergebnisse der Untersuchung zusammengefasst und eingeordnet.

\subsubsection{Analysefähigkeiten und politische Orientierung in Aktion}

Insgesamt zeigt sich, dass die interviewten Schüler*innen in vielfältiger Art und Weise Analysefähigkeiten entwickeln. Einen besonderen Stellenwert hat dabei die Auseinandersetzung mit Informationen. Recherchearbeit und Quellenkritik sind für die Schüler*innen wichtige Elemente ihrer politischen Arbeit. Mit der ,fachbezogene[n] Interpretation von Texten und anderen Medienprodukten" (GPJE 2004, 18) werden damit domänspezifische Fähigkeiten im Feld der politischen Bildung entwickelt. Dabei werden explizit auch jene Quellen kritisch hinterfragt, die den eigenen politischen Positionen eher nahestehen. Für die Interviewten hat ein kritisch reflektierter Umgang mit Informationen einen hohen Stellenwert für ihre politische Arbeit, weil damit das Fundament für eine überzeugende Argumentation geschaffen werde. Glaubwürdigkeit ist für die Jugendlichen ein wertvolles Gut in der politischen Auseinandersetzung und dem Ringen um politische Lösungen. Die Erfahrung mit der politischen Aktion führt dazu, dass die Jugendlichen ihre analytischen Fähigkeiten weiterentwickeln. Dazu gehören auch 
nichtdomänspezifische Fähigkeiten wie das systematische Lesen und Bearbeiten von Texten.

Für sie sind die politische Aktion und die Analyse bestehender gesellschaftspolitischer Verhältnisse untrennbar miteinander verbunden. Die interviewten Jugendlichen betonen, dass sie durch ihr Engagement Nachrichten bewusster wahrnehmen und sich proaktiv mit weiterführenden Informationen befassen. Onlinemedien spielen dabei eine entscheidende Rolle. Die Schilderungen der Jugendlichen bestätigen damit Erkenntnisse breit angelegter quantitativer Untersuchungen wie der JIM-Studie (Feierabend u. a. 2018), der Vodafone-Onlinestudie (Vodafone Stiftung Deutschland 2018) und der Shell-Jugendstudie (Albert u. a. 2019). Vor allem Kinder und Jugendliche informieren sich über gesellschaftliche Fragen und politische Zusammenhänge online (Leven/Utzmann 2019, 287-294). Wenngleich das Vertrauen der jungen Menschen in klassische Medien wie die Tagesschau (84\%) und regionale Tageszeitungen $(77 \%)$ besonders hoch ist (Feierabend u. a. 2018, 591), nutzen sie, um sich über gesellschaftspolitische Themen zu informieren, vor allem digitale Medien (Albert u. a. 2019, 33). Die für die vorliegende Untersuchung interviewten Jugendlichen misstrauen den Informationen, die sie online finden, aber durchaus. Ihren Aussagen ist $\mathrm{zu}$ entnehmen, dass ihnen die Quellenprüfung wichtig ist. Auch diese kritische Grundhaltung gegenüber den Informationen, die sie im Internet finden, lässt sich empirisch mit den Ergebnisse quantitativer Studien bestätigen. Nur knapp 29 Prozent der für die VodafoneOnlinestudie befragten Jugendlichen halten die Informationen und Nachrichten, die sie über soziale Medien erhalten, für ausgewogen (Vodafone Stiftung, 2018, 13).

Die politisch selbstbestimmt aktiven Schüler*innen betonen die Wechselwirkung von politischem Interesse, selbst angeeignetem politischem Wissen und der politischen Aktion, wobei sie dies nicht als linearen Prozess beschreiben. Den Aussagen der Jugendlichen ist ihre Überzeugung zu entnehmen, dass zusätzliches Wissen dazu führe, das politische Interesse zu steigern, und zugleich gesteigertes Interesse dazu beitragen könne, dass die Bereitschaft zur Teilhabe zunehme. Die Aussagen und Erfahrungen stehen damit den empirischen Befunden von Weißeno und Landwehr (2018) gegenüber, die in ihrer Studie keine Korrelation zwischen politischer Partizipation und erhöhtem politischem Wissen erkennen konnten. Das lässt sich auch damit erklären, dass die politisch aktiven Schüler*innen mit dem Zugewinn an politischem Wissen vor allem themenspezifischen Wissenszuwachs in ihren individuellen Interessensfeldern meinen. Die Steigerung des fachlichen Wissens in spezialisierten Themenfeldern wurde bei Weißeno und Landwehr nicht berücksichtigt. Die politische Selbstorganisation und Erfahrungen in der 
politischen Partizipation stärken demnach, dies lässt sich zumindest für die Untersuchungsgruppe rekonstruieren, nicht nur demokratische Werte (van Deth 2017; Quintelier/van Deth 2014), sondern erhöhen auch das politische Interesse und die Bereitschaft sich aktiv einzubringen.

Aus den Erfahrungsberichten der Jugendlichen lässt sich ableiten, dass sich nach eigenem Empfinden die Kompetenzen im Feld der Informationsrecherche, der Informationsverarbeitung und der Quellenkritik durch das Engagement nachhaltig verbessert haben. Sie führen es vor allem darauf zurück, dass sie sich intrinsisch motiviert mit neuen Informationen auseinandersetzen. Die Erfahrungen der Jugendlichen lassen sich demnach in das Verständnis von methodischanalytischer Kompetenzentwicklung von Peter Henkenborg einordnen, der die Bedeutung des Verwaltens und Verarbeitens von Informationen durch dessen Beschaffung, Bewertung und Bearbeitung hervorhebt und auf die Notwendigkeit von Selbstkontrolle und Selbstreflexion in diesem Prozess hinweist (Henkenborg 2005, 303). Diese Grundsätze analytischen Denkens und Handelns gelten sowohl auf individueller als auch auf kollektiver Ebene.

Vor allem im gemeinsamen Austausch zu politischen Themen ist den befragten Schüler*innen solidarische Kritik wichtig. Ein Prozess, der in der Schule zumeist künstlich hergestellt werden muss, beispielsweise durch Feedback-Regeln, die von der Lehrkraft vorgegeben werden, ist für die befragten Jugendlichen teilweise zu einem Selbstverständnis im politischen Diskurs geworden.

Das dieser Arbeit zugrunde liegende Verständnis von sozialwissenschaftlichem Analysieren und politischer Orientierung ist geprägt von einem ganzheitlichen Ansatz, der neben der Auseinandersetzung mit Informationen vor allem auch die Analyse gesellschaftspolitischer Verhältnisse und Konflikte berücksichtigt. Neben der Praxis sozialwissenschaftlicher Methoden ist für die vorliegende Studie auch von Relevanz, inwiefern die politische Arbeit der Jugendlichen verbunden ist mit einer Konflikt- und Gesellschaftsanalyse. Der Konfliktbegriff ist für die politisch aktiven Schüler*innen nicht negativ besetzt. Sie verstehen ihn als komplex und vielschichtig und für die Demokratie unerlässlich. Die Jugendlichen begreifen den politischen Konflikt als wertvollen Bestandteil einer offenen Gesellschaft und nicht als störend. Ihre politische Arbeit hat sie für Konflikte sensibilisiert. Daraus erwächst auch ein aktiver Umgang mit (internen und externen) Konflikten. Das selbstbestimmte politische Engagement ist immer wieder auch Anlass zur Auseinandersetzung mit Macht- und Herrschaftsverhältnissen, aber auch mit der eigenen Rolle in konfliktbehafteten Situationen (beispielsweise Rassismus). Die mannigfaltigen Erfahrungen, die im Kontext der selbstorganisierten politischen Partizipation gesammelt werden, führen zu weitreichenden Selbstreflexionsprozessen. Die Erkenntnisse daraus übertragen sich bei den Schüler*innen 
auch auf den Alltag abseits der politischen Aktion. Der Prozess der politischen Orientierung, der Auseinandersetzung mit Konflikten führt bei den interviewten Jugendlichen zur Entwicklung eines politischen Bewusstseins (Freire 1991 [1971]; Lange 2005, 2008a \& 2008b) und zu einer politischen Subjektwerdung (ausführlich Abschnitt 2.2.1).

Für den komplexen Prozess des sozialwissenschaftlichen Analysierens und Denkens bedarf es neben den hier bereits genannten Fähigkeiten auch der Bereitschaft zur Perspektivenwahrnehmung. Dieses Kompetenzfeld lässt sich kaum von der Ebene des sozialwissenschaftlichen Denkens trennen und ist darüber hinaus eng verbunden mit der politischen Urteilsbildung.

\subsubsection{Politische Aktion - multi- oder monoperspektivisch?}

Perspektivenwahrnehmung im Kontext der Kompetenzentwicklung wird in den Modellen der GPJE (2004) und dem der Autor*innengruppe um Joachim Detjen (2012) nur als Teil der Urteils- und Handlungsfähigkeit formuliert. Die Fachgruppe Sozialwissenschaften (Behrmann u. a. 2004) greift in ihrem Modell die Perspektivenübernahme als eigenständige und relevante Kompetenz für die politische Bildung auf. Die Wahrnehmung anderer Rollen, Perspektiven und Positionen wird als Voraussetzung für gesellschaftliche Interaktionen und als grundlegend für die Entwicklung gemeinsamer Positionen verstanden. Darüber hinaus ermöglicht erst eine Bereitschaft zur Wahrnehmung andere Positionen und Lebensumstände die Perspektiven der Ausgeschlossenen in den Blick zu nehmen (de Moll u. a. 2013; Kleinschmidt u. a. 2019; Eis 2019, Lösch 2019).

In Bezug auf die Entwicklung von Fähigkeiten der Perspektivenwahrnehmung lässt sich zusammenfassend feststellen, dass sich die politisch aktiven Schüler*innen intensiv mit der Bedeutung von Perspektivenwahrnehmung und Multiperspektivität auseinandersetzen. Sie betonen, dass ihnen erst die Auseinandersetzung mit verschiedenen Positionen und Lebenswirklichkeiten die Weiterentwicklung der eigenen Ideen und der eigenen politischen Sichtweisen ermögliche. In Anlehnung an Peter Henkenborg kann konstatiert werden, dass Selbstwahrnehmung und Fremdwahrnehmung offenbar als wechselseitiges Zusammenspiel wirken (Henkenborg 2005, 304) und dieser Prozess, auch wenn er zeitweise an seine Grenzen zu stoßen scheint, von den interviewten Jugendlichen immer wieder reflektiert wird.

Die These von Sibylle Reinhardt $(2014,278)$ politische Aktionen und Bewegungen wären dadurch gekennzeichnet, dass die Perspektive auf die eigene Gruppe reduziert sei, kann zumindest in Bezug auf das Untersuchungssample 
nicht uneingeschränkt bestätigt werden. Die Jugendlichen berichten zwar teilweise von diesem Phänomen, reflektieren diesem Umstand aber auch (selbst)kritisch. Insgesamt scheint es den Jugendlichen, auch durch gruppeninternen und den nach außen offenen Austausch mit Andersdenkenden die Mehrdimensionalität politischer Probleme zu erkennen (siehe hierzu auch: Breit 1999, 387 f.). Besonders spannend ist das Fallbeispiel Katja, die vor ihrem Engagement in einer Antirassismus-AG an ihrer Schule hauptsächlich in einer autonomen Jugendbewegung außerhalb der Schule aktiv war. Sie beschreibt den Homogenisierungseffekt für diese ausschließlich außerschulisch verorte Gruppe und betont, dass ihre Erfahrungen mit der selbstverwalteten politischen Arbeitsgemeinschaft in der Schule ganz andere waren:

\begin{abstract}
Ja, gerade weil die Meinungen so verschieden waren über Politik und über das, was wir eigentlich wollen, weil sonst arbeite ich irgendwie mit Leuten zusammen, die zwar nicht $100 \%$ aber größtenteils alles so ähnlich sehen wie ich. Und es gibt natürlich, man diskutiert auch mal stundenlang über, keine Ahnung Israel und Palästina, aber der Weg, wie irgendwas erreicht werden soll, ist halbwegs ähnlich. Und da sucht man sich auch irgendwie die Leute, mit denen man da konform geht und gut was machen kann. Und jetzt in der Schule ist es halt so, dass alle, die Bock haben, mitmachen. Und da gibt es halt total krasse Unterschiede. Es war total interessant überhaupt mit den Leuten darüber zu reden, was sie so wollen, aber auch zu merken, dass man trotzdem total gut zusammenarbeiten kann und zwar andere Projekte rauskommen, als wenn es mit Leuten ist, mit denen man eher die Meinung teilt, aber es trotzdem total interessant war und irgendwie cool. (KATJA: 396-408)
\end{abstract}

Ein besonderes Potenzial scheinen demnach politische Arbeitsgemeinschaften und Initiativen an den Schulen zu haben. Strukturell bedingt ist die Zusammensetzung der Aktivist*innen hier zumindest in Bezug auf den Aktionskonsens und die politischen Positionen heterogener. Den Erfahrungsberichten der Schüler*innen ist zu entnehmen, dass dies anfänglich als anstrengender empfunden wird, aber insgesamt eine größere Offenheit für vielfältige Perspektiven ermöglicht. Es lässt sich konstatieren, dass die Notwendigkeit politischer Vielfalt einhellig anerkannt wird. Auch politische Gegner*innen werden als relevante und ernst zu nehmende politische Akteur*innen wahrgenommen. Dabei unterscheiden die politisch aktiven Schüler*innen zwischen politisch legitimen und moralisch nachvollziehbaren Positionen. Unabhängig davon, in welchem Kontext (schulisch/außerschulisch) sie aktiv sind, betonen die Jugendlichen, dass sie durch ihr politisches Engagement sensibilisiert wurden für vielfältige Positionen (beispielsweise mehr Empathie) im politischen Diskurs. Aus den Schilderungen der Jugendlichen lässt sich rekonstruieren, dass die verschiedenen Perspektiven auf ihnen besonders wichtige Themen kognitiv präsent (Reinhardt 2004, 4) sind. 
Dennoch: Wenngleich weitaus weniger als zu erwarten wäre (Hillebrand u. a. 2015, 196) führt das außerschulische Engagement von Jugendlichen durchaus zu homogeneren Zusammensetzungen der Akteur*innen und birgt dabei ein höheres Risiko, dass politische Positionen zementiert werden und wenig Raum für neue Impulse bleibt. Grundsätzlich bezeichnen sich die politischen Gruppen als offen. Aber die Schilderungen einzelner Schüler*innen lassen auch erkennen, dass es vor allem bei den außerschulischen Jugendgruppen in Teilen eine Diskrepanz gibt zwischen dem postulierten Selbstverständnis politischer Offenheit und dem tatsächlichen Verhalten. So berichten Jugendliche, die in außerschulischen Gruppen aktiv sind, davon, dass zwar grundsätzlich eine Offenheit für andere Perspektiven bestünde, aber die Bereitschaft, sich den Sichtweisen politisch Andersdenkender tatsächlich zu öffnen, sei nicht immer gleichermaßen etabliert. Bemerkenswert ist, dass die Jugendlichen diesen Effekt selbst wahrnehmen und reflektieren. Sie scheinen diesem Prozess nicht ausgeliefert oder gar davon überwältigt zu sein, wie es bei eher skeptischen Positionen bezüglich real-partizipativen Erfahrungen befürchtet wird (u. a. Oberle 2013; Detjen 2012). Vielmehr setzen sich die Jugendlichen mit diesen Bedingungen kritisch auseinander. Auch in den schulisch verorteten Gruppen politisch aktiver Jugendlicher ist den Schüler*innen die Gefahr bewusst, dass die kollektive Meinungsbildung Einfluss auf die individuelle Urteilbildung nimmt.

Die interviewten Jugendlichen setzen für die Bereitschaft zur Perspektivenwahrnehmung und Perspektivenübernahme auch klare Grenzen: Fremdenfeindliche Hetze, Sexismus und Homophobie sind für die Schüler*innen inakzeptabel. Einhellig wird betont, dass die Schule ein Raum sei, der die Möglichkeit biete, mit vielfältigen politischen Positionen in Kontakt zu kommen. Die Schüler*innen sind überzeugt, dass die Schule sich öffnen sollte für politische Positionierung, für das politische Handeln der Jugendlichen, aber dabei eben auch Grenzen setzen muss, die sich an den Grund- und Menschenrechten orientieren.

Neben analytischen Fähigkeiten und einer damit verbundenen kritisch reflexiven Auseinandersetzung mit Informationen und vielfältigen Perspektiven ist für politische Bildungsprozesse von Bedeutung, welche Auswirkungen diese Fähigkeiten auf die Urteilsbildung der Individuen haben. Mit ihrem politischen Engagement verbinden die Interviewten nicht nur das Ziel, die eigene politische Haltung in der Öffentlichkeit zu vertreten und möglichst viele Menschen davon zu überzeugen. Urteilsbildung und Kritik sind wesentlicher Bestandteil ihrer politischen Arbeit. Sie schreiben dabei ihren Erfahrungen in der politischen Aktion eine herausragende Bedeutung zu. Die kritisch reflektierte Auseinandersetzung mit den durch die politische Aktion geschaffenen Rahmenbedingungen offenbart 
wichtige Voraussetzungen für politische Urteilsbildung. Die Arbeit in autonomen politischen Jugendgruppen scheint damit eine wertvolle Bildungsgelegenheit dazustellen, die zugleich ein hohes Potenzial dafür bietet, die damit verbundenen Erfahrungen im Unterricht aufzugreifen.

\subsubsection{Urteilsbildung und Kritik authentisch erfahren}

Insgesamt wird der Prozess der politischen Interessen- und Urteilsbildung (Hedtke 2020a) im Kontext politischer Aktion von den Schüler*innen als eine authentische Erfahrung empfunden und führt bei ihnen zu einer intrinsisch motivierten Auseinandersetzung mit politischen Problemfeldern. Damit grenzen die Schüler*innen die Urteilsbildung im Kontext ihres Engagements klar von simulierten Verfahren der Meinungsbildung im Unterricht ab. Beispielhaft sei hier auf die Aussagen von FRANZISKA (507-521) und (LEA: 645-657) verwiesen, die herausstellen, dass die politische Selbstorganisation ihr Interesse an politischen Themen vergrößert und ihre Bereitschaft sich kritisch reflektiert mit politischen Themen zu befassen erhöht, weil sie sich frei von Zwängen selbstbestimmt und mit Freude an der Auseinandersetzung mit unterschiedlichen Positionen politischen Themen widmen können. Formale Settings der politischen Bildung sind aber nach wie vor von der Fokussierung auf kommunikative Handlungskompetenz in simulierten Lernsituationen (Detjen u. a. 2012, 83-88) und von Leistungsdruck und Bewertung geprägt.

Wenngleich für die vorliegende Arbeit das Sach- und das Werturteil zusammengedacht werden, liegt der Schwerpunkt der empirischen Untersuchung eher auf der Rekonstruktion politisch-moralischer Urteilsbildung (Reinhardt 2018, 24), weil im Fokus die Erfahrungsberichte der Schüler*innen stehen und nicht das Abfragen von Fachwissen oder Kenntnissen über Statistiken, Definitionen oder dergleichen. Diese wären aber vor allem für die Begründung des Sachurteils von Bedeutung. In Anlehnung an die Überlegungen der Politikdidaktiker Joachim Detjen und Peter Massing zur politischen Urteilsbildung steht demnach eher weniger die Kategorie Effizienz im Fokus, sondern vielmehr die Frage der Legitimität (Detjen 2007; Massing 2003). Das Verständnis von Urteilsbildung findet hier vor allem seinen Ursprung in der Wahrnehmung und Bewusstmachung unterschiedlicher Perspektiven. Erst durch diese Einbeziehung der Perspektiven anderer, vor allem auch der Perspektiven der Ausgeschlossenen oder Marginalisierten (de Moll u. a. 2013, 306; Kleinschmidt u. a. 2019, 416), gelingt ein Urteil, das auf Eigenständigkeit beruht, ,und zugleich durch die Einbeziehung der politischen Perspektiven anderer nicht länger nur subjektiv“ (Juchler 2020, 
233) ist. Die Rekonstruktion der politischen Selbstbildungserfahrungen der interviewten Jugendlichen legte offen, dass die politische Aktion sowohl das Potenzial hat, hier besonders wirkungsmächtige, weil authentische Erfahrungen der Urteilsbildung zu sammeln, als auch zugleich das Risiko birgt, die Offenheit für neue Perspektiven zu verlieren. Auch diese Ambivalenz begründet die Notwendigkeit reale politische Handlungserfahrungen im Sinne der politischen Aktion im Politikunterricht aufzugreifen und die Schüler*innen pädagogisch und politikdidaktisch dabei zu begleiten, die damit verbundenen Widersprüche aufzuarbeiten.

Es lässt sich aus den Erfahrungsberichten rekonstruieren, dass politische Urteilsbildung in vielfältiger Weise durch das Engagement geprägt und zugleich immer wieder auf die Probe gestellt wird. Sie ist damit in einem fortwährenden Prozess und nie abgeschlossen. Der Politikunterricht könnte den Meinungsbildungsprozess aus der politischen Aktion aufgreifen und begleiten. Die Schüler*innen erhoffen sich von gelingendem Politikunterricht, dass sie dabei unterstützt werden, ein pluralistischeres Meinungsbild (weiter) zu entwickeln. Damit das gelingen kann, müssen sich Schule und Unterricht aber öffnen für realpolitische Erfahrungen der Schüler*innen. Es braucht darüber hinaus die Bereitschaft der Schule und der Lehrkräfte als pädagogisch und didaktisch Verantwortliche, mit Kritik und Widerstandbestrebungen (ausführlich und kontroverse Beiträge dazu in Reheis u. a. 2016) als Begleiterscheinung einer politischen Urteilsbildung umzugehen. Denn neben der Urteilsbildung sind die Selbstbildungsprozesse der interviewten, politisch aktiven Schüler*innen zumeist auch geprägt von Kritik an bestehenden Macht- und Herrschaftsverhältnissen. Hervorgehoben wird dabei von den interviewten Jugendlichen unter anderem die Ausbeutung von Mensch und Natur sowie der Machtmissbrauch, beispielhaft begründet an Themen wie der kapitalistischen Wirtschaftsordnung, nationalstaatlichem Denken und dem als willkürlich eingeschätzten Einsatz von Staatsgewalt. Wenn Ziel politischer Bildung sein soll, die Stimme der Ausgeschlossenen hörbar zu machen, zeigen die Berichte der interviewten Jugendlichen, dass ihre Erfahrungswerte aus der politischen Aktion sich als wertvoller Anlass für weiterführende, didaktisch begleitete Bildungsgelegenheiten eignen.

Aktuelle Krisen zeigen deutlich, inwiefern verfestigte gesellschaftliche, v.a. ökonomische Herrschaftsverhältnisse demokratische Verfassungswerte und Gerechtigkeitskonzeptionen untergraben. Fallstudien und Konfliktanalysen als politikdidaktischmethodische Zugänge sollten sich daher nicht auf die Darstellung der Akteure, Interessen und institutionellen Einflussmöglichkeiten im parlamentarischen oder deliberativen Willensbildungsprozess beschränken. (Moulin-Doos/Eis 2016, 137 f.) 
In der Schule, so konstatiert es auch Tilman Grammes, werden Ausdrucksformen des kritischen Charakters beginnend mit „Fragen (Warum?) und Einwänden (Aber!) der Schüler*innen [...] oft überhört oder abgewiesen“ (Grammes 2020, 135). Diese Erfahrung teilen auch die für die vorliegende Studie interviewten Schüler*innen. Die Schule verpasst hier eine einzigartige Chance, politische Urteilsbildung und Kritik an bestehenden Verhältnissen, ausgehend von realen Partizipationserfahrungen, als wertvolle Lernanlässe (Nonnenmacher 2011) aufzugreifen.

\subsubsection{Politische Partizipation und der Wert des politischen Konflikts}

In Bezug auf Partizipationserfahrungen und Konfliktfähigkeit lässt sich zusammenfassend festhalten, dass es den interviewten Jugendlichen nach ihren Aussagen wichtig ist, Öffentlichkeit für die von ihnen ausgewählten politischen Themen zu schaffen. Sie arbeiten nicht konspirativ, sondern wollen mit ihren Aktionsformen einen breiten Diskurs ermöglichen (Eikel 2007, 28; Nonnenmacher 2010, 467). Ziel ist es auf von ihnen identifizierte allgemeinpolitische Missstände (Rassismus, Klimawandel, Ausbeutung, etc.) hinzuweisen, aber auch ihrer Generation eine Stimme zu geben, auch weil sie immer wieder damit konfrontiert werden, dass der Jugend die Fähigkeit zur Artikulation von Kritik an bestehenden Verhältnissen nicht zugetraut beziehungsweise zugestanden wird. Die Erfahrungsberichte bestätigen damit die auch in der Wissenschaft diskutierte adultistische Sichtweiser Erwachsener gegenüber Kindern als schwach und defizitär (Ritz 2013, 165; Schäfer u. a. 2011: 13 f.).

Die politisch engagierten Jugendlichen entwickeln grundlegende Fähigkeiten, die für das politische Arbeiten in Gruppen notwendig sind. Dazu gehören allgemeine Fähigkeiten wie Zeitmanagement, Arbeitsteilung, der respektvolle und wertschätzende Umgang miteinander sowie konstruktives Feedback und solidarische Kritik. Kurzum: Kompetenzen politischer Selbstorganisation. Sie erproben dabei auch Strategien der kollektiven Urteilsbildung und nutzen dafür verschiedene Methoden der Entscheidungsfindung wie das Mehrheitsprinzip oder das Konsensprinzip. Sie lernen dabei gruppenintern, aber auch im Kontext des realen politischen Handelns in Aktionen, mit Konflikten umzugehen und diese zivilisiert zu lösen. Dies beschreiben die Autorengruppe Fachdidaktik (2016, 148) und Sibylle Reinhardt (2018, 20-25) als zentral für die Entwicklung von Konfliktfähigkeit. 
Hervorzuheben ist, dass die befragten Schüler*innen den politischen Konflikt als etwas Wertvolles empfinden und Strategien entwickeln, damit politische Konflikte nicht auch zu persönlichen Konflikten werden. Durch die politische Aktion stärken die Schüler*innen ihre individuellen Handlungskompetenzen in realen und authentischen Erfahrungsräumen des politischen Konflikts und der politischen Willensbildung. Sie entwickeln im Bereich der politischen Grundbildung etwas, das Christine Zeuner $(2013,85)$ als Handlungswissen beschreibt. Nach ihren individuellen Erfahrungen können die Schule und der Politikunterricht ihnen diese Fähigkeiten derzeit nicht vermitteln - das hängt möglicherweise damit zusammen, dass sich der Politikunterricht noch immer allzu häufig auf die institutionenkundlich orientierte Wissensvermittlung fokussiert (siehe hierzu Abschnitt 2.2.2.2).

Ausgangspunkt des politischen Handelns der interviewten Jugendlichen ist oftmals soziales Engagement. Die bewusste Auseinandersetzung mit den politischen Dimensionen im sozialen Handeln ist dabei Triebfeder für das sich entwickelnde politische Bewusstsein und die daraus resultierende politische Teilhabe in Form selbstbestimmter politischer Aktionen. Das Politische in sozialen Problemlagen zu erkennen, ist kein Selbstverständnis, genauso wenig, wie sich politische Partizipation in logischer Folge aus sozialem Engagement heraus entwickelt (Wohnig 2017; Reinhardt 2013, ausführlich in Abschnitt 2.1.2.1). Umso bemerkenswerter ist es, dass alle interviewten Jugendlichen sowohl Erfahrungen im sozialen Engagement als auch in der politischen Aktion haben. Der individuelle Politisierungsprozess und auch die Bereitschaft zur (unkonventionellen) politischen Partizipation sowie letztlich die tatsächlich politische Selbstorganisation sind dabei nicht selten aus der Unzufriedenheit mit einer entpolitisierten sozialen Teilhabe heraus entstanden. Sie manifestiert sich in der Überzeugung, dass soziales Engagement junger Menschen insgesamt eine hohe Anerkennung erfahre und politische Partizipation eher auf Widerstand stoße. Beispielhaft dafür sind auch die Frustrationserfahrungen, die mehrere befragte Jugendliche mit der Schüler*innenvertretung in Verbindung bringen - die sie als unpolitisch und nahezu wirkungslos beschreiben. Diese bewusste und differenzierte Auseinandersetzung mit den Konzepten des sozialen Engagements und der politischen Partizipation wird auch daran deutlich, dass die Schüler*innen darauf verweisen, dass das individuelle Engagement, der Einsatz für individuelle Schicksale zwar richtig und notwendig sei, aber an den politischen Verhältnissen nichts ändere. Exemplarisch dafür steht der Politisierungsprozess von SOPHIE, der sich aus dem Einsatz für einen Mitschüler entwickelte, dessen Abschiebung sie gemeinsam mit anderen Schüler*innen verhindern wollte. Dieses Schlüsselerlebnis war ursächlich für die 
Gründung einer lokalen rassismuskritischen Jugendbewegung, die zunächst hauptsächlich mit Blockaden versuchte, die Abschiebung von Geflüchteten in ihrem Ort zu verhindern. Im Laufe der Zeit transformierte sich das soziale Engagement für die Menschen vor Ort hin zu einem politischen Protest, der darauf gründete, dass die bestehende Abschiebepraxis, beispielsweise mitten im Prozess des Erreichens von Schulabschlüssen oder der Berufsausbildung, als illegitim eingeschätzt wurde. Es ging der Gruppe nicht mehr (nur) darum einzelne Abschiebungen in Kriegsgebiete zu verhindern, sondern die Verhältnisse insgesamt öffentlich zu kritisieren, um ein Umdenken in der Asylpolitik zu erreichen. Sie entwickelten im Kollektiv und ganz individuell Widerstandskompetenz (Schmiederer 1971, 38; Lösch 2013, 173 f.; Oeftering 2016, 126; Moulin-Doos/Eis 2016, 137). Ein Paradebeispiel für die Politisierung aus dem sozialen Engagement heraus und zugleich ein wertvolle Bildungsgelegenheit, die auch die schulische politische Bildung aufgreifen sollte.

Die Jugendlichen nutzen vielfältige Formen der politischen Aktion. Diese zeichnen sich zumeist durch einen unkonventionellen Charakter aus und bewegen sich nicht selten in einem Graubereich zwischen legaler und illegaler Partizipation (siehe Abschnitt 2.1.2). Die Aktivist*innen wählen bewusst Instrumente des zivilen Ungehorsams, wie Platzbesetzungen, Blockaden oder den Schulstreik, um eine politische Öffentlichkeit für ihre Forderungen zu schaffen. Die Politikdidaktikerin Sibylle Reinhardt stellt fest: „,Fridays For Future“ sind Aktionen zivilen Ungehorsams für den Umweltschutz, mit denen die Allgemeinheit aufgerüttelt werden soll." (Reinhardt 2019b, 76) Gleiches gilt auch für die lokalen und bundesweit vernetzten rassismus- oder globalisierungskritischen Jugendbewegungen, die Formate des zivilen Ungehorsams wie Waldbesetzungen, Blockaden oder Streiks, als, in ihren Augen, legitime Mittel des Protests wählen.

Wenngleich Aktionsformen des zivilen Ungehorsams eine besonders breite Öffentlichkeit erreichen und damit auch eine hohe mediale Präsenz, ist das Spektrum der Aktionsformen bei allen Schüler*innen, die für diese Studie interviewt wurden, breiter. Keine*r der Schüler*innen ist singulär im illegalen politischen Protest aktiv. Die Jugendlichen planen Aktionstage, politische Konzerte, Workshops, Lesekreise und Informationsabende. Sie bereiten ihre Aktionen vor und nach. Sie organisieren angemeldete und unangemeldete Demonstrationen, bestreiken ihre Schulen, besetzen öffentliche Plätze und realisieren Protestcamps. Sie blockieren Demonstrationszüge von Rechtsradikalen und Nationalist*innen und versuchen, Abschiebungen zu verhindern. Dabei reflektieren sie die Wirkungskraft und den Zweck der gewählten Aktionsformen. Ihnen ist bewusst, dass sich bestimmte Aktionsformen dazu eignen, einen offenen Diskurs anzuregen, und andere Formate eher zur politischen Willensbildung beitragen. Bestimmte Aktionen dienen dazu, Mitstreiter*innen zu finden, andere eher dazu, sich mit bereits 
politisierten Menschen zu vernetzen. Zu den Erkenntnissen über den Stellenwert von kritischer (Selbst)Reflexion in den Bildungserfahrungen, die Jugendliche im Kontext politischer Aktionen und politischer Selbstorganisation erleben, wird am Ende dieses Kapitels ausführlicher eingegangen.

Aus den Schilderungen der Jugendlichen lässt sich schließen, dass sie mit ihrem individuellen und kollektiven politischen Handeln nicht immer eine vermeintlich richtige Lösung für politische Konflikte artikulieren wollen. Wichtiger sei ihnen, dass Anregungen zum selbstständigen Denken geschaffen werden. Daher sollen die von ihnen gewählten politischen Aktionsformen möglichst offen für alle sein, die sich einbringen wollen. Den politisch aktiven Schüler*innen ist durchaus bewusst, dass sie die globalen Schlüsselprobleme der Gegenwart mit ihren Aktionsformen nicht lösen können. Sie wollen dennoch versuchen, immer mehr Menschen dafür zu sensibilisieren.

Wenngleich die interviewten Schüler*innen dieses Kernziel betonen, ist doch festzustellen, dass sie in ihren politischen Positionierungen zu bestimmten Themen sehr gefestigt sind. Die Schule und der Politikunterricht könnten genau hier ansetzen, die Erfahrungen aus der politischen Aktion aufgreifen und die teils komplexen Argumentationsstrukturen der Jugendlichen in den formalen Bildungsraum transferieren. Beispielhaft arbeitet Sibylle Reinhardt die Vielschichtigkeit politischer Urteilsbildung im Kontext der Klimaproteste auf:

\begin{abstract}
Aber für konkretes klimaschützendes Handeln muss das Postulat in die konkrete Komplexität unseres Lebens übersetzt werden. Diese politischen Entscheidungen sind überhaupt nicht mehr klar und eindeutig und einfach, denn sie berühren die LebensInteressen vieler Menschen, Regionen und Institutionen. Ein plötzlicher Ausstieg aus der Braunkohle zum Beispiel würde einige Regionen und die in ihnen lebenden Menschen ihrer Existenz berauben, Mobilität auch per Auto ist für viele Arbeitende und für ländliche Regionen zurzeit unerlässlich, die Versorgung mit sicherer Energie ist eine Grundlage für unser Leben [...]. Die Demokratie als Konflikt-System ist gefordert und wird durch den Anstoß gefördert. (Reinhardt 2019b, 77)
\end{abstract}

Emotionale Dimensionen, genauso wie gruppendynamische Effekte, die mit unkonventionellen politischen Aktionen eng verbunden sein können, bergen die Gefahr, dass es zu einer „Verengung der Perspektive“ (Hufer 2013b, 149) bei den Jugendlichen kommt. Doch das ist kein Grund, politische Aktion als wertvolle Bildungsgelegenheit für die formale politische Bildung auszuschließen. Im Gegenteil: Es bietet die Möglichkeit, Politik und das Politische als Kern demokratischer Gesellschaften erfahrbar zu machen.

Das Konflikthafte und der Dissens sind der Kern der Demokratie und damit nach Chantal Mouffe $(2020,16)$ konstitutiv für Gesellschaften. Selbstbestimmte 
und selbstorganisierte politische Aktionen von Jugendlichen sind authentische Erfahrungsräume dafür. Sollte das Konflikthafte in diesem Prozess verloren gehen, kann das zugleich Ausgangspunkt für didaktisch begründete Bildungssettings in formaler politischer Bildung sein.

\subsubsection{Politisierung zwischen Frustration und Selbstwirksamkeit}

Grundlage für die Rekonstruktion der Bildungserfahrungen sind im Wesentlichen zentrale Kompetenzmodelle der politischen Bildung. Diese dienen im Zuge der Analyse des Materials zur strukturierten Auswertung. Im Prozess der qualitativen Inhaltsanalyse wurde allerdings schnell deutlich, dass diese deduktiven Kriterien zur Systematisierung politischer Selbstbildung im Kontext politischer Aktion der aktiven Jugendlichen den komplexen Erfahrungen der Interviewten nicht gerecht werden. Eine im Vorfeld der Untersuchung nur in Ansätzen antizipierte Dimension politischer Bildungserfahrung in der politischen Aktion ist der Einfluss von Anerkennungs- und Frustrationserfahrungen sowie das Selbstwirksamkeitserleben auf die Entwicklung der politischen Persönlichkeit und des politischen Bewusstseins der engagierten Jugendlichen. Die damit verbundenen Erfahrungen stellen allerdings ein zentrales Element der Erfahrungsberichte aller interviewten Schüler*innen dar. Daher wurden im Zuge der mehrstufigen Materialanalyse induktive Kategorien wie „Frustration“, „Anerkennung“, „Wir-Ihr-Konstruktionen“ und „Selbstwirksamkeit“ in das Kodiersystem aufgenommen.

Wenngleich aus den Berichten der Interviewten insgesamt abzuleiten ist, dass ihnen die politische Arbeit viel Freude bereitet, so beschreiben sie auch vielfältige Frustrationserfahrungen. Insgesamt offenbart die Analyse der Erfahrungsberichte politisch aktiver Schüler*innen eine große Ambivalenz zwischen Erfahrungen der Selbstwirksamkeit und dem Gefühl politischer Ohnmacht. Sie berichten euphorisiert von den Erfahrungen in Vorbereitung, Durchführung und Reflexion ihrer politischen Aktivitäten und schildern zugleich vielfältige Erlebnisse, die zu Frustration führen und ein Gefühl der Machtlosigkeit manifestieren.

Die Ebenen der Frustrationserfahrungen sind dabei vielschichtig. Auf der einen Seite führt die zeitlich und inhaltlich komplexe Auseinandersetzung mit gesellschaftspolitischen Schlüsselproblemen wie Rassismus, Ausbeutung und Klimawandel zu Überwältigungsmomenten. Sie teilen die Sorge, diesen Herausforderungen mit ihrem Engagement nur unzureichend begegnen zu können. Darüber hinaus scheint vor allem das selbstorganisierte und nicht-institutionalisierte 
politische Engagement der Jugendlichen davon geprägt zu sein, dass es an Steuerungsmechanismen mangelt, die eine Überforderung durch Überlastung einzelner Akteur*innen verhindern. Kurz gesagt: Die Schüler*innen, die für die vorliegende Untersuchung interviewt wurden, leiden teilweise an Überarbeitung. Diese in den Interviews besonders häufig identifizierbare Ebene der Frustration scheint vor allem dem Umstand geschuldet, dass sich bei den politisch aktiven Schüler*innen das Gefühl verfestigt, selbstbestimmtes politisches Engagement sei unerwünscht. Diesen Eindruck artikulieren die Schüler*innen auf verschiedenen Ebenen, die im Folgenden mit der Trias Mikro-, Meso- und Makroebene systematisiert werden. Die Mikroebene steht dabei für Frustrationserfahrungen in Bezug auf das (zwischenmenschliche) Beziehungsgeflecht mit der eigenen Familie, Freund*innen Mitschüler*innen oder den Lehrkräften. Die Mesoebene meint Frustrationserfahrungen, die mit institutionellen Strukturen und Hierarchie- bzw. Machtkonstellationen in der Schule als Sozialisationsraum verbunden sind. Und mit der Makroebene sind Frustrationserfahrungen im Konflikt mit der Ordnungsmacht als Konfrontationspunkt innerhalb des politischen Systems gemeint. Dies bezieht sich auf Konflikte mit der Polizei, die das staatliche Gewaltmonopol umsetzt, aber auch Konflikte mit staatlichen Organen der Verwaltung, die beispielsweise verantwortlich sind für die Realisierung von Naturschutzverordnungen.

Auf der Mikroebene schildern die Schüler*innen frustrierende Erlebnisse mit ihren Familien, Freund*innen, Mitschüler*innen und Lehrkräften. Während der Konflikt mit den eigenen Eltern eher als natürlicher Prozess der Identitätsfindung nur am Rande thematisiert wird, erscheinen reale Erfahrungen der sozialen Exklusion in Schule oder dem Klassenverband für die interviewten Jugendlichen prägender zu sein. Es entwickelt sich eine Wir-Ihr-Konstruktion im Verhältnis zu den Mitschüler*innen und Lehrkräften. Politische Initiativen oder Arbeitsgemeinschaften scheinen kein selbstverständlicher Teil der demokratischen Schulkultur zu sein, sondern sind vielmehr aufwändig erkämpfte Freiräume. Aus den Schilderungen lässt sich schließen, dass die politisch aktiven Schüler*innen sowohl von Mitschüler*innen als auch von Lehrkräften den Eindruck vermittelt bekommen, dass ihre Aktivitäten die vermeintlich eingespielten Abläufe in der Schule oder gar den Schulfrieden störten. Ganz konkret erleben sie es in Form von Ablehnung durch ihre Mitschüler*innen, fehlender Unterstützung durch Lehrkräfte bis hin zu Verboten und der Androhung und Umsetzung von Repressionen.

In Bezug auf die Mesoebene ist aus den Erfahrungsberichten der Schüler*innen vor allem eine hohe Unzufriedenheit mit Formen der Alibi-Partizipation oder Scheinbeteiligung abzuleiten. Die Strukturen repräsentativer Demokratie, die in der Schule durch die Einrichtung von Klassensprecher*innen und der Schüler*innenvertretung eingeübt werden sollen, verbinden einige Aktivist*innen 
eher mit Ordnungsaufgaben und fehlenden realen Mitbestimmungsrechten. Damit decken sich ihre Erfahrungen mit empirischen Befunden quantitativer Studien zur Partizipation von Schüler*innen (u.a. Achour u.a. 2020, 168 ff.; Stange 2010, 18 ff.; Schneider u.a. 2009, 15 ff. - siehe hierzu auch Abschnitt 2.1.3).

Auf der Makroebene wird der Konflikt vor allem in der Auseinandersetzung mit der staatlichen Ordnungsmacht gesehen. Auch hier entwickelt sich eine Wir-Ihr-Konstruktion. Eine Minderheit der Befragten sieht in der Polizei mit dem staatlichen Auftrag der Durchsetzung von Recht und Gesetz sogar einen Erfüllungsgehilfen der politischen Gegner*innen. Das wird vor allem in Bezug auf illegale politische Protestaktionen deutlich. Die Konflikte, von denen die Schüler*innen berichten, stehen zumeist in Verbindung mit Protestformen des zivilen Ungehorsams wie unangemeldeten Demonstrationen, Besetzungen und Blockaden. Als Folge aus den Frustrationserfahrungen und vor allem der fehlenden Aufarbeitung und pädagogischen Begleitung dieser Erlebnisse können sich bei politisch aktiven Schüler*innen auch Radikalisierungstendenzen entwickeln. Klaus-Peter Hufer beschreibt als eine der Grenzen des Lernens in und mit politischen Aktionen die Gefahren, die mit der eigenen Betroffenheit verbunden sind. Dies führe dazu, dass ,,alternative Entscheidungs- und Handlungsmotive aus dem Blick geraten" (Hufer 2013b, 149). Darauf bezugnehmend wird das Potenzial der Einbindung von Erfahrungen aus der politischen Aktion in formale Bildungssettings besonders deutlich. Möglicherweise kann eine handlungsorientierte Didaktik der politischen Bildung, die sich auch für die Erfahrungen aus der politischen Aktion öffnet, dazu beitragen, dass derartige Frustrationserfahrungen aufgearbeitet werden und damit ein Gegengewicht zu Radikalisierungstendenzen und Ohnmachtserfahrungen geschaffen wird.

Die Mitwirkung in politischen Aktionen, vor allem aber die damit verbundene politische Selbstorganisation hat auch eine nachhaltig positive Wirkung auf das politische Bewusstsein und das Selbstwirksamkeitsempfinden der interviewten Schüler*innen. Einige Schilderungen der Schüler*innen lassen darauf schließen, dass sie in der Auseinandersetzung mit Familie, Freund*innen, Schüler*innen und Lehrkräften nicht nur negative Erfahrungen machen. Sie erleben teilweise Bestärkung und Unterstützung durch ihre Eltern, erhalten Rückendeckung durch ihre Freund*innen und finden neue Freund*innen in der politischen Bewegung. Manche Lehrkräfte unterstützen sie in ihrem Engagement und heben dieses Engagement gar als besondere Leistung hervor. Viel wichtiger als die Erfahrungen der Anerkennung durch andere scheint für die Schüler*innen allerdings die Kraft zu sein, die sie aus den politischen Aktionen und der Selbstorganisation ziehen. Hervorzuheben ist, dass sie dieses Gefühl nicht nur auf Erfolgsmomente 
zurückführen. Sie sehen auch einen Wert in der Bewältigung von Rückschlägen. Individuelle Erfahrungen mit unkonventionellen Partizipationsformen, aber auch das Wirken im politischen Kollektiv scheinen ihnen beim Umgang mit Ohnmachtserfahrungen zu helfen. Damit bestätigen diese Erfahrungsberichte die folgende These von Hartmut Rosa:

Das Einbringen ermöglicht den Bürger*innen die durch nichts zu ersetzende Erfahrung politischer Selbstwirksamkeit. Durch ihre Stimme sind sie mit den anderen und dem Gemeinwesen verbunden und haben Anteil an der kollektiven Gestaltung der Welt. (Rosa 2019, 165)

Die politische Aktion im Kollektiv vermittelt den Jugendlichen das Gefühl, nicht allein zu sein mit ihrem Engagement, mit ihrer Überzeugung, etwas bewegen zu wollen. Den interviewten Jugendlichen ist dabei aber auch das emotionale Dilemma bewusst, in dem sie sich befinden, weil sie die Welt weder allein noch in ihren politischen Gruppen nachhaltig verändern können und doch der Wunsch nach Veränderung ihr Antrieb ist. Ihre Rolle als politische Subjekte in einer komplexen politischen Welt scheinen die Jugendlichen auch durch die Erfahrungen in der politischen Aktion zu finden. Für die Schüler*innen ist politische Arbeit Teil ihrer Persönlichkeitsbildung. Es macht ihnen Spaß, sich zu engagieren, und sie empfinden die politische Arbeit als einen Teil ihrer politischen Identität.

Das politische Engagement und dabei nicht zuletzt auch die konstruktive Auseinandersetzung mit Frustrationserfahrungen stärkt das Selbstwirksamkeitsempfinden der Interviewten. Sie identifizieren ihre eigenen Stärken und Schwächen. Die Bewältigung von Rückschlägen, aber auch die Erfolgserlebnisse, die Bestätigungen, die Suche und das Finden der eigenen politischen Identität und der individuellen Fähigkeiten prägen die Schüler*innen. Sie werden selbstbewusst, ihr Mut, sich öffentlich politisch zu positionieren, steigt, und sie lernen, dem gesellschaftlichen Anpassungsdruck zu widerstehen.

Die Jugendlichen empfinden ihre politische Arbeit als sinnstiftend. Diese Suche nach Sinnstiftung ist maßgeblich für ein subjektorientiertes Verständnis politischer Bildung (Lange 2008a, 432). Dieser sinnstiftende Charakter gibt ihnen Sicherheit im öffentlichen politischen Meinungsaustausch. BEN fasst diese Erfahrungen mit dem Begriff „Reifeprozess“ (BEN: 25) zusammen. Neben der politischen Urteilsbildung und der Befähigung zur politischen Artikulation eigener Positionen lässt sich aus den Interviews auch die Erkenntnis ableiten, dass ihnen die politische Aktion das Gefühl gibt, vermeintlich feste politische Strukturen aufbrechen zu können. Mit ihrem politischen Engagement halten die Schüler*innen den Ohnmachtserfahrungen etwas entgegen. 
Unkonventionelle, selbstbestimmte und selbstorganisierte politische Aktion bedeutet für die interviewten Schüler*innen ein permanentes Wechselspiel aus Frustration und Anerkennung, Selbstbewusstsein und Selbstzweifel. Sich dieser Ambivalenz der politischen Aktion zu stellen, sie zu reflektieren und ihr zu begegnen, ist im Kontext politischer Bildung eine wertvolle Bildungserfahrung, die der Politikunterricht und die demokratische Schule den jungen Menschen nicht abnehmen können. Es ist eine Erfahrung, die auch nicht künstlich erzeugt werden kann, aber mit Konzepten einer handlungsorientierten Didaktik der politischen Bildung pädagogisch zu begleiten ist. Mit pädagogischer und fachdidaktischer Expertise können die Denkhorizonte, das politische Bürgerbewusstsein (Lange 2005, 2008a, 2008b) und damit der politische Bildungsprozess der jungen Menschen angeregt werden.

\subsubsection{Reflexion als Stärke auf allen Ebenen der Kompetenzentwicklung}

Um die Forschungsergebnisse einordnen zu können, muss abschließend noch einmal der Fokus auf (Selbst)Reflexion als zentrales Element der rekonstruierten politischen Bildungserfahrungen gelenkt werden. Der Diskurs über den Wert realer politischer Partizipationserfahrungen wird auch über den vermeintlichen Gegensatz von Reflexion und Aktion geprägt. Für Klaus-Peter Hufer kann dieses Begriffspaar zu einem ,sich gegenseitig ergänzenden Wechselspiel, aber auch zum antagonistischen Gegensatz werden“ (Hufer 2013b, 147).

Im Zuge der Rekonstruktion der politischen Selbstbildungserfahrungen der interviewten Schüler*innen wurde deutlich, dass Reflexion für die jungen Menschen auf allen Ebenen politischer (Selbst)Bildung einen großen Stellenwert hat. Sie reflektieren den Einfluss ihres politischen Engagements auf ihren Umgang mit Informationen und berücksichtigen dabei auch die Wirkung ihrer politischen Grundhaltung auf die Auswahl von Quellen. Deutlich wird das bereits im Kontext des sozialwissenschaftlichen Analysierens. Politische Aktion und Selbstorganisation bedeuten für die Schüler*innen nicht nur Aktivismus. Wenngleich in der Regel die politische Aktion und dabei vor allem der Regelübertritt, zum Beispiel in Form des zivilen Ungehorsams, ein besonders großes öffentliches Interesse erzeugen, so stellt es den Schilderungen der Jugendlichen zufolge nur einen Bruchteil der politischen Arbeit dar. Wir sehen demnach nur die Spitze des Eisberges (Haunss 2016, 35) aller potentiellen politischen Selbstbildungserfahrungen. Politische Aktion bedeutet für die Jugendlichen vor allem auch die Vorund Nachbereitung politischer Aktivitäten. Und diese Prozessphasen sind geprägt 
von Kritik und Selbstreflexion. Sie befassen sich dafür beispielsweise intensiv mit fremden, aber auch den eigenen Texten (bspw. auf der Internetpräsenz der Gruppe, in den Sozialen Medien, Flyern, etc.). Insbesondere das politische Handeln von HELGE sei hier exemplarisch noch einmal hervorgehoben. Er engagiert sich unter anderem in einer Gruppe, die einen politischen Instagram-Kanal verantwortet. Hier werden politische Texte veröffentlicht, Debatten angestoßen und in der Kommentarfunktion unter jedem Post begleitet. Die kollaborative Erstellung der Texte, die Reflexion der Inhalte, aber auch die Auswertung der Diskussion unter dem veröffentlichten Text zeugen von einem hohen Abstraktionsvermögen und der Bereitschaft, mit politischem Protest mehr zu verbinden als nur die Verbreitung politischer Botschaften. Insgesamt berichten die politisch aktiven Schüler*innen einhellig davon, dass sie sich nicht nur intensiver mit Informationen befassen und breitgefächerter nach glaubwürdigen Quellen suchen, sondern die Ergebnisse ihrer Recherche auch kritischer reflektieren, als sie es vor ihrem politischen Engagement getan haben. Sie betonen dabei immer wieder, dass ihr Antrieb intrinsisch geprägt sei und sich politische Bildungs- und Erfahrungsräume ergeben würden, die ihnen der Unterricht allein nicht ermögliche:

Und im Unterricht ist es meistens so, da muss man ja auch manchmal Gruppenarbeit oder so reflektieren. Aber da ist es meistens so als Zwang dargestellt und man soll aufschreiben, was gut war und was schlecht war. Aber wenn man sich irgendwie selbst mehr damit befasst und es nicht als Zwang vorgegeben ist, hat das so viel mehr Effekt. [...] [D] ]ann wird es erstens von vielen einfach nicht so ernst genommen, und zweitens hat man dann, glaube ich, nicht so den großen Lerneffekt. Und deswegen ist es einfach viel wichtiger, außerhalb des Unterrichts zu lernen und selbst zu reflektieren. (LEA: 645-653)

Die Jugendlichen reflektieren den Prozess der Urteilsbildung und damit auch den Einfluss ihres sozialen Umfeldes auf die eigene Meinungsbildung. Besonders deutlich wird das an der These, mit der FRANZISKA Rechtfertigungsstrategien der Menschen für ihr individuell (un)moralisches Handeln begründet. Sie ist überzeugt, dass das ,gesellschaftliche Sein, das gesellschaftliche Bewusstsein des Menschen bestimm [e] " (FRANZISKA 403). Sie ist sich sicher, dass gesellschaftliche Veränderung nur mit einer Bewusstseinsveränderung der Menschen erreicht werden könne. Das erfordert aber eine besonders hohe Reflexionsfähigkeit in Bezug auf das eigene Urteilen und Handeln.

Im politischen Handeln beginnt der Reflexionsprozess weit vor der Umsetzung politischer Aktionen. Schon in der Vorbereitung bei Plenums- und Arbeitsgruppentreffen nutzen und reflektieren die politisch aktiven Schüler*innen Instrumentarien wie (doppelt)quotierte Redelisten oder Entscheidungsprinzipien, wie 
das Mehrheits- (FRANZISKA: 624-641) oder das Konsensprinzip (SOPHIE: 236-249). Aber auch der konkrete Einsatz von Partizipationsformen wird (selbst)kritisch reflektiert. Den Jugendlichen ist bewusst, dass sich manche Formen des Protests dafür eignen, Menschen aufzurütteln oder zum Nachdenken anregen. Mit anderen Aktionsformen werden konkrete, lokale und zeitlich begrenzte Ziele verfolgt - beispielsweise die Verhinderung einer Abschiebung oder der Bau einer Autobahn. Wieder andere Aktionsformen dienen eher der Stärkung des Gruppengefühls. Dabei bleibt auch der Einsatz illegaler Aktionsformen des zivilen Ungehorsams nicht unreflektiert: Schulstreiks, Demonstrationsblockaden oder die Verhinderung von Abschiebungen werden nicht als bloße Mittel zum Zweck verstanden. Sie werden argumentativ in einen ganzheitlichen Kontext von Machtverteilung und Machtmissbrauch eingebettet.

Abschließend sei darauf verwiesen, dass auch die Wirkung und die Legitimität des politischen Engagements von den Schüler*innen hinterfragt wird. Die Positionen, beispielsweise zum Einsatz der richtigen Mittel im politischen Protest, sind dabei durchaus unterschiedlich.

Jugendlichen in autonomen Protestgruppen, vor allem auch im Klimaprotest, wird bisweilen vorgeworfen, dass sie aus einer Position vermeintlicher moralischer Überlegenheit argumentieren, hauptsächlich selbstreferentiell agieren und dabei viele Perspektiven aus dem Blick verlören. Umso bemerkenswerter erscheint die Antwort mehrerer Schüler*innen auf die Frage, wie sie die Welt gestalten würden, wenn sie die Entscheidungsmacht über das gesellschaftspolitische Leben hätten. Im ersten Impuls präsentierten sie keine Konzepte direktdemokratischer Entscheidungsprozesse oder klimaneutraler Wirtschaftskreisläufe. Vielmehr brachten sie mit dem ersten Impuls zum Ausdruck, dass sie die Vorstellung für abwegig hielten, dass sie allein Gesellschaft gestalten würden. Beispielhaft dafür steht die Antwort von LAURENZ: „Also, erst mal möchte ich nicht die Entscheidungsgewalt haben, als Alleiniger, weil es wieder dieser Idee der Demokratie widersprechen würde, logischerweise. "(LAURENZ: 115-117)

Mit der vorliegenden explorativen Studie ist es gelungen, Prozesse politischer Selbstbildung aus den Erfahrungsberichten politisch aktiver Schüler*innen zu rekonstruieren. Daraus können keine generalisierbaren Aussagen über das Potenzial politischer Aktionen für Selbstbildungsprozesse von Kindern und Jugendlichen abgeleitet werden. Vielmehr steht im Mittelpunkt der vorliegenden Untersuchung, welche Formen, Formate und Fertigkeiten und Fähigkeiten politischer Bildung Jugendliche im Kontext des selbstbestimmten realen politischen Handelns erfahren. Dabei wurden Anknüpfungspunkte herausgearbeitet, die offenlegen, inwiefern reale politische Partizipationserfahrungen und das dabei erworbene politische Handlungswissen als Bildungsgelegenheiten auch in der 
formalen politischen Bildung aufgegriffen werden können. Es kann festgestellt werden, dass die Auseinandersetzung mit den Bildungserfahrungen in der politischen Aktion wechselseitig fruchtbar sein kann, für die Erfahrungsprozesse der Jugendlichen selbst und für die Bildungssettings der formalen politischen Bildung - wenn sich die Akteur*innen dafür öffnen.

Die Schüler*innen, das ist aus den Erfahrungsberichten abzuleiten, würden sich eine pädagogische und fachdidaktische Begleitung ihrer Bildungserfahrungen in der politischen Selbstorganisation durch die demokratische Schule und den Politikunterricht wünschen. Sie kritisieren den institutionenkundlichen Charakter des Politikunterrichts und die fehlende Offenheit der Lehrkräfte und der Schule insgesamt für ihre individuellen politischen Handlungserfahrungen. Die strukturierte Analyse und Auswertung der Erfahrungsberichte politisch aktiver Schüler*innen offenbarte, dass die politische Aktion einen Ausgangspunkt für vielfältige politische Bildungserfahrungen darstellt. Zugleich könnten die Schüler*innen in der Auseinandersetzung und Reflexion der Bildungserfahrungen von den Erkenntnissen jahrzehntelanger Forschung im Feld der Politikdidaktik profitieren. Der Politikunterricht kann beispielsweise unterstützend wirken, um den komplexen Prozess des analytischen Denkens und der pluralistisch geprägten Urteilsbildung der Jugendlichen weiterzuentwickeln. Aber auch die pädagogische Dimension politischer Bildung kann, beispielsweise bei der Aufarbeitung von Frustrationserfahrungen, eine wertvolle Begleitung der politischen Selbstbildung sein. Auch die gemeinsame Reflexion von Handlungserfahrungen in unkonventionellen Partizipationsformen kann für alle Beteiligten ein wertvoller Lernanlass sein. So könnte gelingen, was Hermann Giesecke schon in den 1970er-Jahren für die politische Bildung einforderte. Lernen und Handeln würde eng miteinander verknüpft und aus einem ,antinomischen Verhältnis ein dialektisches“ werden, indem auf „Phasen der Aktion [...] solche der Reflexion“ (Giesecke 1971, 26) folgen. In jedem Fall liegt in der Berücksichtigung der politischen Handlungserfahrungen von Kindern und Jugendlichen im Kontext der schulischen politischen Bildung eine große Chance, Politik und das Politische erfahrbar zu machen und gemeinsam zum Thema unterrichtlicher Lernprozesse zu machen. Wie dies gelingen kann, wird hier nicht abschließend beantwortet werden können. Im folgenden Kapitel werden allerdings Denkanstöße und Anregungen für die Entwicklung einer handlungsorientierten Politikdidaktik formuliert. 
Open Access Dieses Kapitel wird unter der Creative Commons Namensnennung 4.0 International Lizenz (http://creativecommons.org/licenses/by/4.0/deed.de) veröffentlicht, welche die Nutzung, Vervielfältigung, Bearbeitung, Verbreitung und Wiedergabe in jeglichem Medium und Format erlaubt, sofern Sie den/die ursprünglichen Autor(en) und die Quelle ordnungsgemäß nennen, einen Link zur Creative Commons Lizenz beifügen und angeben, ob Änderungen vorgenommen wurden.

Die in diesem Kapitel enthaltenen Bilder und sonstiges Drittmaterial unterliegen ebenfalls der genannten Creative Commons Lizenz, sofern sich aus der Abbildungslegende nichts anderes ergibt. Sofern das betreffende Material nicht unter der genannten Creative Commons Lizenz steht und die betreffende Handlung nicht nach gesetzlichen Vorschriften erlaubt ist, ist für die oben aufgeführten Weiterverwendungen des Materials die Einwilligung des jeweiligen Rechteinhabers einzuholen.

(c) (9) 